\title{
Organization of the Cytoskeleton: Studies in Microfluidic Drops
}

\author{
Dissertation \\ for the award of the degree \\ 'Doctor rerum naturalium' \\ of the Georg-August-Universität Göttingen
}

within the doctoral program

Göttingen Graduate School for Neurosciences, Biophysics, and Molecular Biosciences (GGNB)

of the Georg-August University School of Science (GAUSS)

submitted by

\section{Christian Dammann}

from Stade

Göttingen 2014 


\title{
Thesis committee:
}

\author{
Prof. Dr. Sarah Köster
}

Institute for X-Ray Physics

Georg-August-Universität Göttingen

Dr. Thomas Burg

Department for Biological Micro- and Nanotechnology

Max Planck Institute for Biophysical Chemistry, Göttingen

Prof. Dr. Eberhard Bodenschatz

Department of Fluid Dynamics, Pattern Formation and Nanobiocomplexity Max Planck Institute for Dynamics and Self-Organization 


\section{Members of the examination board:}

Reviewer: Prof. Dr. Sarah Köster

Institute for X-Ray Physics

Georg-August-Universität Göttingen

Second Reviewer: Dr. Thomas Burg

Department for Biological Micro- and Nanotechnology

Max Planck Institute for Biophysical Chemistry, Göttingen

\section{Further members of the examination board:}

Prof. Dr. Eberhard Bodenschatz

Department of Fluid Dynamics, Pattern Formation and Nanobiocomplexity

Max Planck Institute for Dynamics and Self-Organization

Dr. Florian Rehfeldt

Third Institute of Physics

Georg-August-Universität Göttingen

Prof. Dr. Jean-Christophe Baret

1. University of Bordeaux, Centre de Recherche Paul Pascal (CNRS)

2. Max Planck Institute for Dynamics and Self-Organization,

'Droplets, Membranes \& Interfaces'

Prof. Dr. Jörg Enderlein

Third Institute of Physics

Georg-August-Universität Göttingen

\section{Date of oral examination:}

24.03.2014 



\section{Contents}

1 Introduction 1

2 State of the art and theoretical background 5

2.1 Cytoskeletal proteins .................... 5

2.1.1 Intermediate Filaments . . . . . . . . . . 6

2.1.2 Organization in the cell ............. 7

2.1 .3 Organization in vitro . . . . . . . . . . . . . . 9

2.2 Biopolymers as polyelectrolytes . . . . . . . . . . . . . 13

2.2.1 Ionic solutions . . . . . . . . . . . . . . . 13

2.2.2 Manning counterion condensation . . . . . . . . . 15

2.2 .3 Ion competition . . . . . . . . . . . . . . 17

2.2.4 Attraction mechanisms . . . . . . . . . . . . 19

2.3 Microfluidic techniques . . . . . . . . . . . . . 22

2.3 .1 Flow on small scales . . . . . . . . . . . . . . . 22

2.3.2 Droplets as microcontainers . . . . . . . . . . . . 23

2.3.3 Diffusion and mixing . . . . . . . . . . . . 25

3 Materials and methods 29

3.1 Preparation of vimentin protein . . . . . . . . . . . . . . 29

3.1.1 Vimentin mutants . . . . . . . . . . . . . . . . 29

3.1.2 Production and labeling ............... 32 
CONTENTS

3.1.3 Dialysis and assembly .................. 33

3.2 Fabrication of microfluidic devices . . . . . . . . . . . . . . 34

3.2.1 Photolithographic methods . . . . . . . . . . . 34

3.2 .2 Device production $\ldots \ldots \ldots \ldots$

3.3 Emulsion production in microfluidic channels . . . . . . . . . 37

3.4 Fluorescence microscopy . . . . . . . . . . . . . . . . . . . 37

4 Microfluidic devices for protein studies 39

4.1 Device C: Concentration dependence . . . . . . . . . . . . . . . 39

4.1.1 Motivation ................... 39

4.1 .2 Device design and function . . . . . . . . . . . . 40

4.1.3 Application to vimentin aggregation . . . . . . . . . 43

4.2 Device T: Time-lapse studies . . . . . . . . . . . . . . 45

4.2 .1 Concept ..................... 45

4.2.2 Device design and function . . . . . . . . . . . 46

4.2.3 Trapping characteristics ................ . 48

5 Vimentin in drop devices $\quad 51$

5.1 Mixing characteristics . . . . . . . . . . . . . . . 51

5.2 Drop volume changes and concentration steadiness . . . . . . . . 56

5.3 The challenge to encapsulate vimentin in drops . . . . . . . . . . . 59

6 Data analysis $\quad 63$

6.1 Concentration determination in the device $C \ldots \ldots 3$

6.2 Network morphologies in time-lapse studies . . . . . . . . . . . 68

6.2.1 From raw data to network morphologies . . . . . . . . . 68

6.2 .2 Binarization of images . . . . . . . . . . . 70

6.2.3 The convex hull of networks . . . . . . . . . . 75 
7 Interaction of vimentin and multivalent ions $\quad 79$

7.1 Concentration dependence . . . . . . . . . . . . . . . . . . 79

7.2 Time-lapse studies . . . . . . . . . . . . . . . . 82

7.2.1 Qualitative aggregation analysis . . . . . . . . . 83

7.2.2 Details on aggregation over time . . . . . . . . . 86

7.3 Time-scales in the aggregation process . . . . . . . . . . . . . . 92

7.4 Studies without drops . . . . . . . . . . . . . . . 97

7.4.1 Vimentin studied on cover glasses . . . . . . . . . . . . 97

7.4.2 Networks with vimentin mutants . . . . . . . . . . . . . 99

8 Polyelectrolyte nature of vimentin 103

8.1 System parameter . . . . . . . . . . . . . . . . . . . . 103

8.2 Manning Counterion condensation . . . . . . . . . . . . . . 105

8.3 Competitive ion condensation . . . . . . . . . . . . . 106

8.4 Robustness of the analysis . . . . . . . . . . . . . . . 109

8.5 Strength of counterion correlation . . . . . . . . . . . . . . 111

8.6 The tail of vimentin . . . . . . . . . . . . . . . . . . 112

9 Discussion 115

9.1 Microfluidic techniques . . . . . . . . . . . . . . 115

9.1.1 The benefit of device C . . . . . . . . . . . . 115

9.1.2 The benefit of device T . . . . . . . . . . . . . . 117

9.1.3 Time-stability of salt concentrations . . . . . . . . . . . 119

9.1.4 Effects of mixing and inhomogeneity in concentration . . . . 121

9.1.5 The challenge of protein aggregation in small channels . . . 122

9.1.6 Are microfluidic drops just miniaturized test tubes? . . . . . 123

9.2 Interaction of vimentin with multivalent ions . . . . . . . . . . . 124

9.2.1 Filament networks . . . . . . . . . . . . . . 125

9.2 .2 The onset of aggregation . . . . . . . . . . 127 
CONTENTS

9.2.3 Assumptions for the polyelectrolyte nature . . . . . . . . 132

9.2.4 The role of the tail $\ldots \ldots \ldots \ldots$

9.2.5 Implications for the cytoskeleton . . . . . . . . . . . 136

10 Outlook

11 Summary and conclusions

Abbreviations and symbols

Bibliography

List of publications 165

Erklärung 167

Lebenslauf

Danksagung 


\section{1 \\ Introduction}

Eukaryotic cells are the fundamental constituents of all multicellular organisms. Each cell has a complex internal 'machinery' that allows the cell to fulfill various functions. This cellular machinery has a complex build-up and consists of numerous components, such as DNA, proteins, organelles, small molecules and ions. Many of these components are densely packed in the cytoplasm, where they fulfill their cellular functions. One protein system found in the cytoplasm is the so-called 'cytoskeleton'. The cytoskeleton acts as the scaffold ('skeleton') of the cell and provides mechanical integrity. Additionally, the cytoskeleton, for example, serves as tracks for motor proteins and is involved in the process of cell motility. There are three main fibrous protein systems that belong to the cytoskeleton: actin filaments, microtubules and intermediate filaments. Each of these fiber-forming protein systems has mechanical, chemical and electrostatic properties. The interplay of all these properties determines the organization of these filamentous systems in the cell.

The mechanisms behind the organization of the cytoskeleton are of fundamental interest because of the central role of the cytoskeleton in the cell. However, the cytoskeleton is a complex system in the cell due to the tremendous number of 'players' in the cytoplasm. Therefore, experiments of reduced complexity are established in which purified parts of the cytoskeleton are investigated in vitro. This way, the chemical, mechanical or electrostatic properties can be studied systematically and regulatory mechanisms are revealed step-by-step. One experimental concept for studies on the electrostatic properties of cytoskeletal proteins is to investigate their behavior in the presence of oppositely charged ions ('counterions'). Given the right conditions, these counterions mediate the attraction of the 
like-charged protein filaments.

Microfluidic drops have been established as an experimental tool for in vitro studies in various fields, among them cell and protein research. These drops are small containers with a volume of typically a few nano- or picoliters and are sometimes described as 'miniaturized test tubes'. They are produced in microfluidic devices in vast numbers by merging an aqueous and an oil phase in tailor-made channels. During the process of drop production, a biological sample in the aqueous phase is encapsulated into the drops, where it can be manipulated in manifold ways. As a result, this microfluidic method becomes a tool for life sciences.

Here, we design such drop-based microfluidic tools to encapsulate purified vimentin intermediate filaments. We use the drops to investigate the electrostatic properties of vimentin filaments in the presence of counterions. The drops allow us to study the influence of these ions on vimentin with respect to ion type and concentration in a defined way. We are also able to record the dynamics of the aggregation process. Moreover, we shed light on the polyelectrolyte nature of vimentin filaments.

In chapter 2, we present the scientific background of the complex cytoskeleton build-up in the cell and we give a literature review of experimental studies on purified cytoskeletal components in vitro. Subsequently, we discuss the polyelectrolyte properties of biopolymers, which will help to understand the interaction of vimentin and cations later on. We then describe flow characteristics on small scales because this provides the theoretical basis for the microfluidic tools we use.

In chapter 3, we discuss the fundamental procedures of our experimental approach. Subsequently, we describe the microfluidic devices that were tailored for the investigations of vimentin aggregation (chapter 4 ). First, we discuss the device, which we use to monitor the salt concentration dependence of vimentin aggregation ('device C'). Second, we show how another device gives access to imaging the dynamics of vimentin aggregation ('device $\mathrm{T}^{\prime}$ ). We discuss the characteristics of these microfluidic techniques with respect to their application in vimentin protein research (chapter 5). A detailed description of the data analysis procedures is given in chapter 6. Subsequently, we apply the data evaluation methods to our observational results of vimentin protein aggregation in the drops (chapter 7). 
In addition to the experimental studies on vimentin, we investigate the electrostatic properties of the protein from a theoretical point of view. For this purpose, we discuss the polyelectrolyte nature of vimentin in chapter 8 . The findings from our experiments and theoretical calculations are then discussed in detail in the discussion found in chapter 9. In the first part of this discussion, we reflect on the properties of the microfluidic techniques we have developed and applied. In the second part, we combine the observational findings of vimentin aggregation with the theoretical analysis of the polyelectrolyte nature of this protein. In doing so, we shed light on the underlying fundamental principles of vimentin filament aggregation in the presence of multivalent ions. 



\section{2 \\ State of the art and theoretical background}

\subsection{Cytoskeletal proteins}

The protein network in the cell that spans over the cytosol in all eukaryotic cells is called the cytoskeleton (Fig. 2.1a). [2] One main function of the cytoskeleton is to provide the mechanical integrity of the cell, but it has also other important functions such as cell motility [103], cell division (Fig. 2.1b) [65], signal transduction or transport mechanism as it serves as tracks for motor proteins. The three main filamentous constituents of the cytoskeleton are microtubules (MTs), fila-

a)

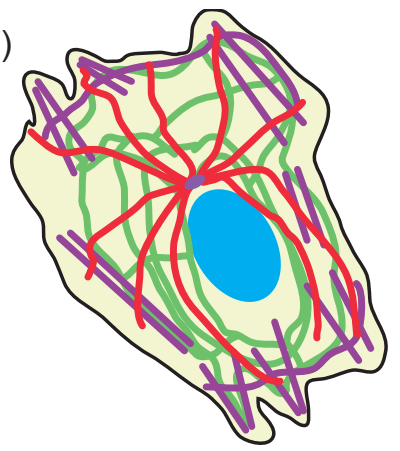

F-actin

microtubules

intermediate filaments

nucleus b)

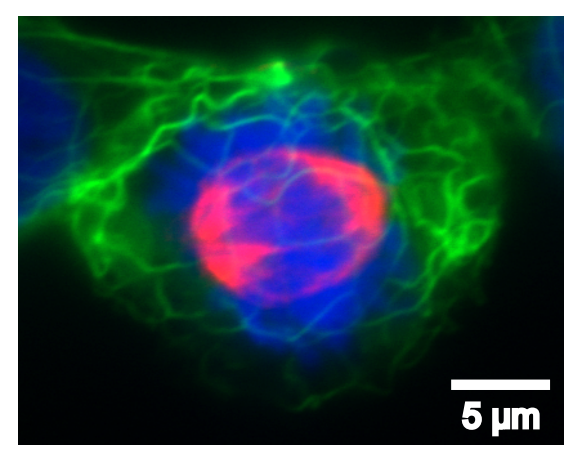

Figure 2.1: The cytoskeleton. a) Sketch of the cytoskeleton that consists of the three major fibrous protein systems F-actin, microtubules and intermediate filaments (adapted from [2]). b) Fluorescence micrograph of a eukaryotic cell during cell division showing its keratin network (green), the nucleus (blue) and microtubules in the mitotic spindle colored red. (SK8/18-2 kidney carcinoma cell expressing keratins [142], courtesy of Britta Weinhausen) 
mentous actin (F-actin), and a protein family called intermediate filaments (IFs). Whereas the organization and mechanical properties of MTs and F-actin is known quite well $[2,15,66,70,108]$, there is much less known about intermediate filaments. However, intermediate filaments gain more and more attention, among other things, due to their connection to human diseases. [41,94,128] For instance, there is a connection of mutations in neurofilaments to Parkinson disease [41] or of vimentin to cataracts that evoke eye lens opacification [88]. IFs gather their name from the fact that their filament diameter is with approximately $10 \mathrm{~nm}$ in between that of F-actin ( $\approx 7 \mathrm{~nm}$ ) and microtubules ( $25 \mathrm{~nm}$ ). [2]

\subsubsection{Intermediate Filaments}

The members of the IF family are found in animal cells. [54] They clearly differ from F-actin and MTs. [2] First, their assembly does not need an energy carrier like adenosine triphosphate (ATP) for F-actin or guanosine triphosphate (GTP) for MTs. Second, they are apolar and therefore do not serve as tracks for mo-

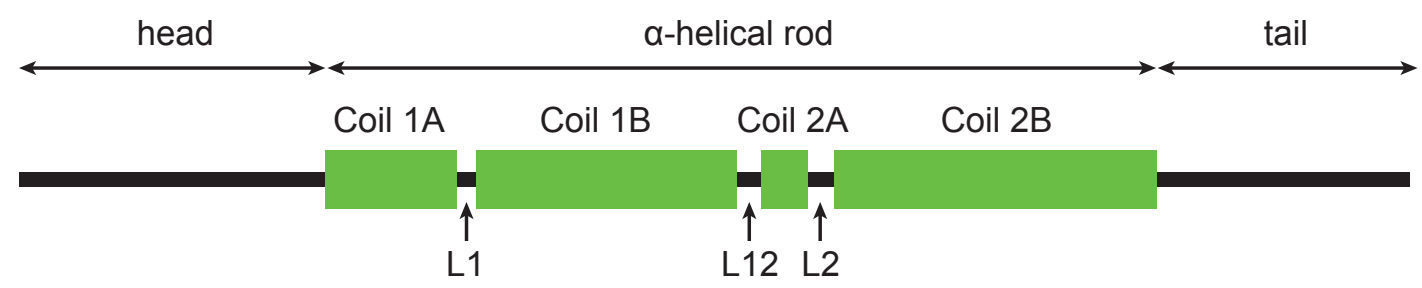

Figure 2.2: Intermediate filament structure. The shared tripartite structure of the intermediate filament protein family consists of the flexible and length-variable head $(\mathrm{N}-$ terminus) and tail (C-terminus) domains and the rod domain. The rather stiff rod domain consists of the $\alpha$-helical subdomains $1 \mathrm{~A}, 1 \mathrm{~B}, 2 \mathrm{~A}$ and $2 \mathrm{~B}$ which are connected via linker sequences L1, L12 and L2. (For reference, see [54] for example)

\begin{tabular}{rlll}
\hline class & & example & occurrence \\
\hline I/II & epithelial & (acidic/neutral-basic) keratins & epithelial cells, hair, nails \\
III & vimentin-like & vimentin & cells of mesenchymal origin \\
& & & $e . g$. fibroblasts, smooth muscle cells \\
& & desmin, paranemin, synemin & muscles \\
IV & axonal & neurofilament proteins & neurons \\
V & nuclear & lamins & envelope of nucleus \\
VI & & filensin, phakinin & fiber cells (lens) \\
\hline
\end{tabular}

Table 2.1: Intermediate filament classes. Classification of the intermediate filament multigene family given for important IF examples. (From: $[2,41,64,135]$ ) 
tor proteins like myosins (F-Actin) or kinesins (MTs). Third, there are far less intermediate filament associated proteins (IFAPs) known that are specific for IFs compared to F-actin or MTs (see below). [33,78] Fourth, the building blocks for F-actin (globular actin) and MTs (tubulin) are globular proteins which are highly conserved. [54] In contrast, IFs are a large protein family including over 70 proteins with a very diverse amino acid sequence. Nevertheless, they all have a tripartite build-up consisting of a head, rod and a tail domain. [65] The stiff rod domain is $\alpha$-helical and highly conserved in the IF family (Fig. 2.2). The head and tail are variable in length and in comparison to the rod domain very flexible. $[41,54,128]$ There is a classification of the intermediate filaments according to features like assembly behavior, occurrence in the organism or location in the cell (Tab. 2.1).

\subsubsection{Organization in the cell}

In the cytoplasm, there are many different proteins, small ions, organelles and other biopolymers. This huge number of intracellular 'players' makes the organization of the cytoskeleton very complex. [2] However, important regulation mechanisms are known. Proteins that are associated to at least one of the three main cytoskeletal proteins have the ability to organize the cytoskeleton. For actin and microtubules, there are a lot of proteins that regulate attachment, growth, bundling and other manipulation mechanisms. [2,38,78,80] In contrast, far less intermediate filament associated proteins (IFAPs) are known that bind specifically to IFs. $[46,78]$ One example of these specific binding proteins is filaggrin that bundles keratin IFs. [33] A family of proteins associated to IFs are plakins which are important cytolinker proteins. [73] These linker proteins bind to all major cytoskeletal filament systems and have other interaction partners like focal adhesions or spectrins. One prominent protein of the plakin family is plectin which is found in almost all tissues. In Fig. 2.3, the role of plectin in fibroblasts is demonstrated as an example for the versatile role of plectin as cytolinker. [120] It is believed that plectin is essential to the orchestrations of the cytoskeletal network and therefore to the mechanical properties of cells. [140]

As the cytoskeletal proteins have distinct electrostatic properties, it is important to investigate to which extent small ions organize the cytoskeleton in the cell. In Tab. 2.2, typical ion concentrations in the cytosol of vertebrate cells are 


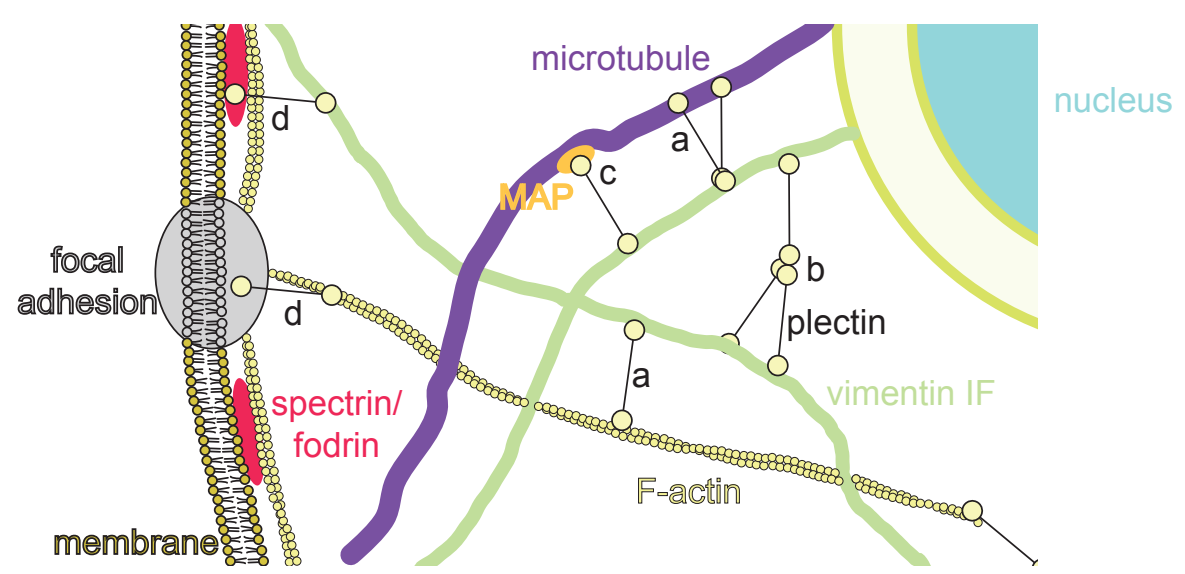

Figure 2.3: The versatile role of plectin in fibroblasts. $[120,140]$ Plectin acts as a mechanical linker and can a) connect to actin stress fibers, vimentin and microtubules, b) bridge longer distances by self-connectivity, c) attach to microtubule associated proteins (MAPs), d) link cytoskeletal filaments to focal adhesions and to the submembrane spectrin/fodrin network. (Sketch adapted from [140] with permission of the Journal of Cell Science)

given. As we will see in the next section, ions can directly orchestrate the architecture of the fibrous cytoskeletal proteins, like F-actin or MTs, when studied purified in vitro. However, in the cell, processes related to the ionic balance are highly integrated into many cellular functions (for review see [72]). Cells have to have certain concentrations to keep their metabolism intact. To this end, they regulate their volume and therefore the concentration of components decreases (cell swelling) or increases (cell shrinkage). One process for this regulation is the transport of ions through ion channels in the membrane, which is also coupled to water transport via aquaporins over the membrane. As a consequence, the ionic balance changes all the time and differs locally. This can lead to a concentration

\begin{tabular}{ccc}
\hline Ion & & Concentration [mM] \\
\hline potassium & $\mathrm{K}^{+}$ & 139 \\
sodium & $\mathrm{Na}^{+}$ & 12 \\
chloride & $\mathrm{Cl}^{-}$ & 4 \\
bicarbonate & $\mathrm{HCO}_{3}^{-}$ & 12 \\
negatively charged proteins & & 138 \\
magnesium & $\mathrm{Mg}^{2+}$ & 0.8 \\
calcium & $\mathrm{Ca}^{2+}$ & $<0.0002$ \\
\hline
\end{tabular}

Table 2.2: Ions in cells. Typical ion concentrations in the cytosol in vertebrate cells. (From: [78]) 
gradient that is used during cell migration for which the cells have to mediate strong cytoskeletal reorganization. During this migration, calcium ions stimulate gelsolin which leads to the depolymerization of F-actin at the rear end of the moving cell, whereas the polymerization of actin takes place at the leading edge. Ions are also important in extracellular fluids. For instance, biopolymers leave the cell, when a cell dies. [60] It is found that in these cases extracellular ions mediate the formation of large structures of DNA or F-actin in the extracellular environment. In summary, small metal ions are a regulation mechanism of the cytoskeletal proteins. However, they have to be considered as part of the complex metabolism of the cell.

\subsubsection{Organization in vitro}

The assembly and organization mechanisms of various biopolymer systems have been studied in vitro. We first discuss the assembly behavior of the intermediate filament vimentin as it is the IF most expressed in cells of mesenchymal origin. [45] Subsequently, we focus on important findings in the field of biopolymer aggregation.

\section{Assembly mechanisms and network formation of vimentin}

IF protein monomers associate to coiled-coil dimers in the rod domain (example: Fig. 2.4). However, not all IFs form homodimers, which means that the

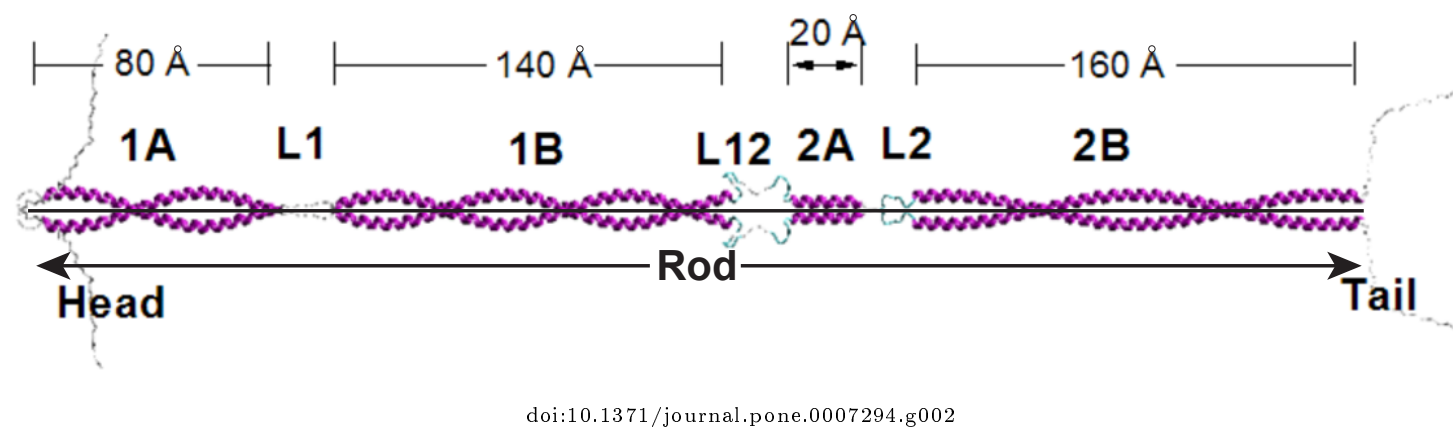

Figure 2.4: Structure of vimentin. Coiled-coil structure in a vimentin dimer. Both monomers show the conserved IF tripartite structure as shown in Fig. 2.2. The nonhelical head domain of wild-type human vimentin monomers consists of 77 amino acids, the rod domain of 334 amino acids that are connected via the non-helical linkers and the non-helical tail domain of 55 amino acids. [52] (Figure adapted from: [100] with CCAL) 
a) monomer

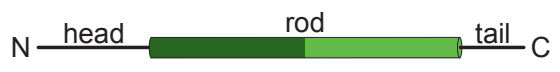

b) dimer

c) tetramer

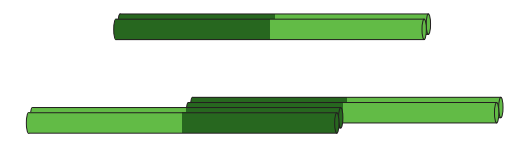

d) unit-length filament (ULF)

e)mature filament

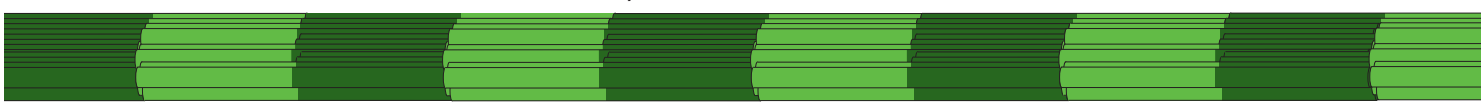

Figure 2.5: Sketch of the vimentin assembly process from monomers to filaments. Two vimentin monomers (a) form a dimer (b). Two dimers associate in a half-staggered manner and become a tetramer (c). d) When the ionic strength in the protein solution is increased, usually eight tetramers assemble laterally to form a unit-length filament (ULF). The ULFs assemble longitudinally to a filament. The filament becomes mature in a radial compaction step (e). The head and the tail regions are not shown from b to e. (See e.g. [25,97] for reference)

identical monomers can associate to dimers. [54] Vimentin assembles as a homodimer as shown in Fig. 2.4. Two dimers associate to tetrameric vimentin in a half-staggered manner with the heads pointing in opposite directions (Fig. $2.5 \mathrm{~b} / \mathrm{c}$ ). This tetramer formation takes already place in low ionic strength conditions. From this state, the assembly to a mature filament is triggered as the ionic strength rises, e.g. the potassium chloride concentration of a tetramer solution is increased. The tetramers form filaments in a hierarchical process [97]: They associate laterally to form unit-length filaments (ULFs), which usually consist of eight tetramers and are approximately $17 \mathrm{~nm}$ in diameter and $60 \mathrm{~nm}$ in length (Fig. 2.5d). Several of these short filament precursors anneal to filaments. Finally, in a radial compaction step, the filament becomes mature as its diameter is reduced to approximately $10 \mathrm{~nm}$ (Fig. 2.5e). Mature vimentin filaments have a persistence length of $2 \mu \mathrm{m}$ and - as the filaments also reach these length scales - vimentin is a semiflexible polymer. [93]

The number of tetramers forming a ULF can vary. [52,87] In the literature, this phenomenon is described as 'polymorphism'. Additionally, the filament properties also depend on the ion types that are present during the assembly. Brennich et al. demonstrate that the properties of vimentin filaments differ when they are assembled in the presence of potassium ions only compared to an assembly with magnesium ions only. [25]

Beyond the steps that lead to the assembly of vimentin subunits to mature filaments, the behavior of these filaments in the presence of multivalent ions was 
investigated. Vimentin filaments build entangled networks, given a sufficient protein concentration. In rheology experiments, the influence of multivalent ions on these networks has been investigated. It is found that magnesium ions act as effective cross-linker of these networks and thereby stiffen them. $[69,76,77]$ Lin et al. hypothesize that parts of the flexible tail domain of vimentin (Fig. 2.4) play an important role for the cross-linking of vimentin networks in the presence of magnesium ions. [76] The importance of IF sidearms has been confirmed for neurofilaments, which have comparatively long sidearms. These filaments connect via a 'hand-shake' mechanism of their side arms (details are discussed in Sec. 9.2.4). [16] A further influence of charged ions on vimentin filaments is found in light scattering experiments. These reveal the ability of polylysine, which carries multiple positive charges, to mediate bundle formation of vimentin. [130] In summary, these in vitro experiments demonstrate that ions have two effects on vimentin protein: They initiate the assembly to vimentin filaments and they give rise to the bundling and network formation of the filaments.

\section{Influence of multivalent ions on other biopolymers}

There have been many studies of F-actin, MTs and IFs in vitro (see [56] for review). Here, we give an overview of the findings that are related to the interaction of cellular polymers with small ions. F-actin, MTs, IFs and DNA are biopolymers that all share a net negative charge, i.e. they are polyanions. [60,145] That is why their interaction with cations as counterions is important. In general, the presence of multivalent ions can lead to structures of the biopolymer filaments that are described with expressions like 'bundles', 'networks', 'aggregates' or other (more specific) expression in the literature. All these expressions convey that multivalent ions mediate a morphological rearrangement of the biopolymers.

Light scattering experiments have shown that F-actin undergoes lateral association leading to bundle formation in the presence of multivalent ions. Bundling is observed once the concentration of multivalent ions exceeds a threshold concentration $c_{\mathrm{th}}$. The threshold concentration $c_{\mathrm{th}}$ follows the general trend that the higher the cation valency is, the smaller is $c_{\mathrm{th}}$. For instance, the threshold values, at otherwise identical experimental parameters, are approximately $c_{\mathrm{th}}: 5.5 \mathrm{mM}$ $\left(\mathrm{Co}^{2+}\right), 7 \mathrm{mM}\left(\mathrm{Mn}^{2+}\right), 20 \mathrm{mM}\left(\mathrm{Ca}^{2+}\right), 27 \mathrm{mM}\left(\mathrm{Mg}^{2+}\right)$ or $3 \mathrm{mM}\left(\mathrm{Co}\left(\mathrm{NH}_{3}\right)_{6}^{3+}\right)$. This example also shows that not only the valency but also other effects like ion radius and hydration are important. [131] Additionally, the onset of aggregation 
can depend on the ionic strength of the solution. In experiments where the ionic strength is mainly determined by the monovalent ion concentration, a higher ionic strength requires a higher concentration of the cationic bundling agents to mediate bundling. [130] X-ray studies shed light on some interesting details of F-actin attraction. F-actin can be organized into raft-like structures with increasing divalent ion concentration before they take a bundle configuration at higher salt concentration. [146] This shows the potential of small ions to induce sophisticated network architectures. Using fluorescence imaging techniques, aster-like F-actin networks are observed in the presence of magnesium ions. [57] In X-ray diffraction studies, Angelini et al. have directly measured how counterions are organized in bundled F-actin. The counterions show a wave-like density along the F-actin bundles. [5]

When the cation concentration reaches a critical concentration, MT aggregation is observed for many multivalent ions, leading to MT bundles. The bundles are necklace-like for divalent ions and hexagonal arranged for cations of higher valency. Interestingly, magnesium ions are, like monovalent ions, unable to mediate aggregation of MTs, whereas other divalent cations are able to. [91]

DNA has a distinct polyelectrolyte character and is therefore an important biopolymer to compare cytoskeletal filaments with. [21,22] In the presence of multivalent ions, DNA undergoes substantial compaction when around $90 \%$ of its surface charge is neutralized. The preferred structures are toroid-like. In aqueous solution this requires a counterion valency of $3+$. This requirement for aggregation is in agreement with the concept of counterion condensation theory (see Sec. 2.2.2). Interestingly, it is observed that aggregated DNA can be resolubilized again once the cation concentration becomes very high. [102]

In summary, many in vitro studies demonstrate that ions have a marked influence on biopolymers. We find no experimental examples in which monovalent ions mediate attraction between polyelectrolytes. A theoretical explanation for this observation is given in Sec. 2.2.4. There is often a threshold cation concentration needed until attraction between the like-charged biopolymers sets in. In this way, diverse morphologies of aggregates can emerge, ranging from tight bundles to structures of sophisticated architecture. 


\subsection{Biopolymers as polyelectrolytes}

Polymers which carry charges are called polyelectrolytes. [59] The important biopolymers like DNA, microtubules, filamentous actin or intermediate filaments are also polyelectrolytes carrying net negative charges. [60,145] As described in Sec. 2.1.3 the influence of oppositely charged ions (counterions) can lead to strong attraction phenomena between polyelectrolytes. At first sight this is counterintuitive as the two like-charged polyelectrolytes should electrostatically repel each other. [22] Therefore, theories were developed to explain how polyelectrolytes interact with counterions and under which circumstances counterions can mediate attraction between polyelectrolytes. [145]

\subsubsection{Ionic solutions}

When a salt is dissolved in a solvent, the resulting solution is overall electrically neutral. Here, we sketch the fundamental properties of these ionic solutions. [59] The Poisson equation

$$
\nabla^{2} \Psi=-\frac{\varrho}{\epsilon \epsilon_{0}}
$$

connects the electrostatic potential $\Psi$ with the charge density $\varrho$ of the ions. [136] In this equation $\epsilon_{0}$ is the dielectric permittivity and $\epsilon$ the relative permittivity of the solution. The local concentration $n_{i}$ of the $i$ th ion species of valency $z_{i}$ has the Boltzmann weight

$$
n_{i}=n_{\mathrm{bi}} \exp \left(-\frac{z_{i} e \Psi}{k_{\mathrm{B}} T}\right),
$$

where $e$ is the elementary charge, $k_{\mathrm{B}} T$ is the thermal energy at a temperature $T$ and $n_{\mathrm{b} i}$ is the bulk concentration of the $i$ th ion type. Considering that $\varrho=\sum_{i} z_{i} e n_{i}$, we obtain the non-linear Poisson-Boltzmann equation from Eq. 2.1 and 2.2:

$$
\nabla^{2} \Psi=-\frac{e}{\epsilon \epsilon_{0}} \sum_{i} z_{i} n_{\mathrm{b} i} \exp \left(-\frac{z_{i} e \Psi}{k_{\mathrm{B}} T}\right)
$$

This equation is important as it is a fundamental approach to describe electrostatic effects in ionic solutions. [44] However, it is a mean-field approach, which 
neglects thermal fluctuations and ion correlations (see also Sec. 2.2.4). [136] In the example of a potassium chloride solution, Eq. 2.3 becomes in the one dimensional case [23]:

$$
\frac{\mathrm{d}^{2} \phi}{\mathrm{d} x^{2}}=\sinh \left(\phi / l_{\mathrm{D}}^{2}\right) .
$$

Herein, $\phi=e \Psi / k_{\mathrm{B}} T$ is the normalized electrostatic potential and $l_{\mathrm{D}}:=1 / \sqrt{8 \pi n_{\mathrm{KCl}} l_{\mathrm{B}}}$ the Debye-length. In this definition the Bjerrum-length $l_{\mathrm{B}}$ is introduced. The Bjerrum length is the distance at which the thermal energy $k_{\mathrm{B}} T$ and electrostatic energy of two elementary charges are equal:

$$
l_{\mathrm{B}}:=\frac{e^{2}}{4 \pi \epsilon_{0} \epsilon k_{\mathrm{B}} T} .
$$

At room temperature $\left(20^{\circ} \mathrm{C}\right)$ in water this gives [131]

$$
l_{\mathrm{B}}=0.71 \mathrm{~nm} .
$$

This parameter is of great importance as it indicates that for charge separation distances of $l<l_{\mathrm{B}}$ the electrostatic interactions are important and otherwise thermal fluctuations dominate.

For small potentials $\phi$, Eq. 2.4 can be approximated by $\sinh \phi / l_{\mathrm{D}}^{2} \approx \phi / l_{\mathrm{D}}^{2}$ (DebyeHückel approximation). Then the solution of Eq. 2.4 becomes

$$
\phi=\phi_{0} \exp \left(-x / l_{\mathrm{D}}\right) .
$$

In this solution the Debye length indicates the decay of the potential of a charge and describes at which distance the charge is screened by its surrounding ions. [23]

In the general case of several ion species in a solution the Debye-Hückel approximation is also applicable. Then the Debye length is more generally defined by [23]

$$
l_{\mathrm{D}}:=\sqrt{\frac{1}{8 \pi l_{\mathrm{B}} I}} .
$$


In this definition the ionic strength is introduced:

$$
I:=\frac{1}{2} \sum_{i} n_{\mathrm{bi}} z_{i}^{2}
$$

\subsubsection{Manning counterion condensation}

The Poisson-Boltzmann equation provides a way to describe the distribution of ions around polyelectrolytes. However, it can easily lead to mathematical difficulties. Other approaches emerged that treat problems differently. [123] One of them is the theory of counterion condensation as pioneered by Onsager, Manning [81] and Oosawa [95]. The polyelectrolyte is considered as a cylinder with radius $R$ and linear charge density $\lambda$ (Fig. 2.6a), the energy of a counterion of valency $z$ is investigated. [47] The electrostatic energy cost $\Delta W$ for a counterion to move away from the surface of an assumingly infinitely long cylinder by the distance $r-R$ with $r>R$ is given by

$$
\Delta W=\frac{z e \lambda}{2 \pi \epsilon \epsilon_{0}} \ln (r / R)
$$

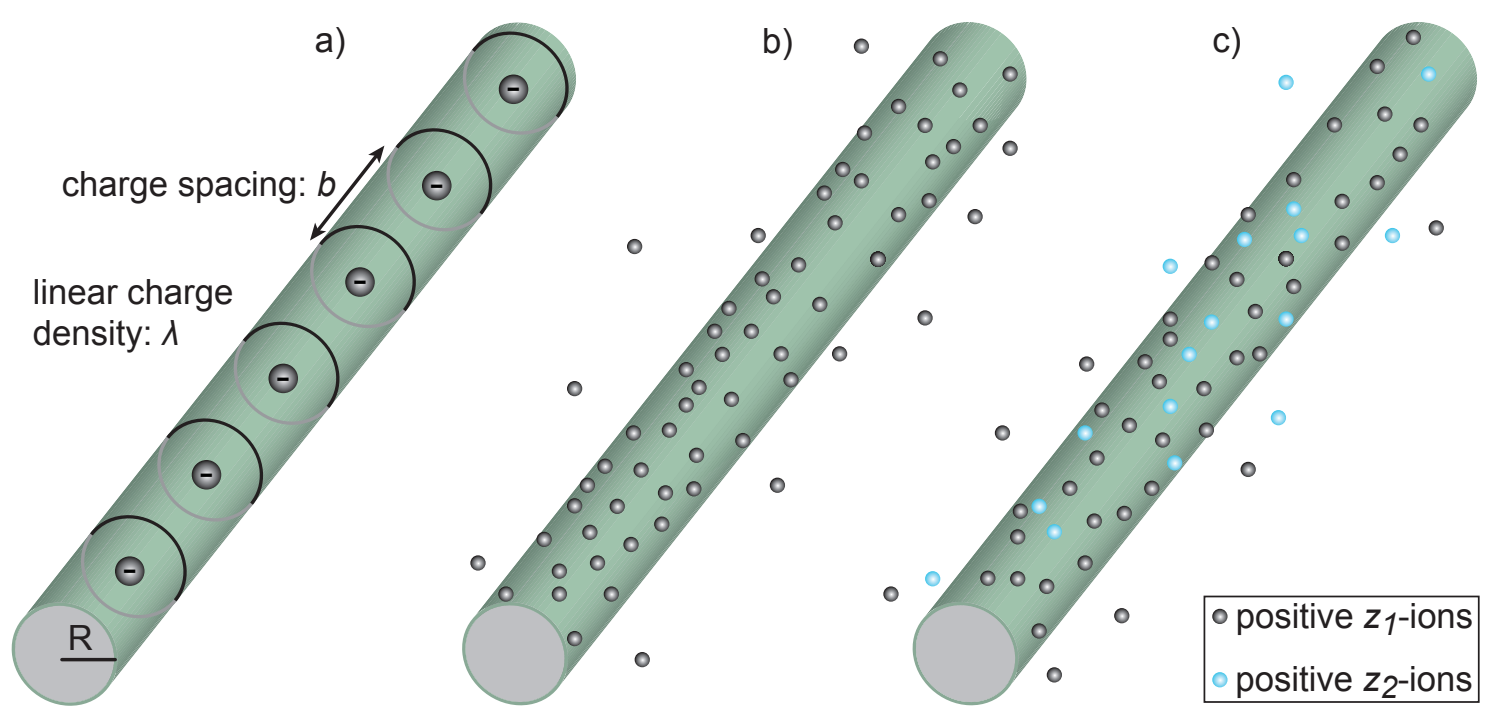

Figure 2.6: Model for a polyelectrolyte. a) Model for a polyelectrolyte as a cylinder with radius $R$. b) Condensation of counterions of valency $z_{1}$ onto the cylinder. c) Competitive binding of counterions in the presence of a second counterion species of valency $z_{2}$. 
Further, the ion gains entropic energy by diverging from the surface

$$
\Delta W_{S}=-k_{\mathrm{B}} T \ln \left(\pi r^{2} / \pi R^{2}\right)
$$

as the accessible space increases. The counterion remains bound (condensed) given $\Delta W>\Delta W_{S}$. Introducing the dimensionless 'Manning parameter':

$$
\xi:=l_{\mathrm{B}} / b
$$

with length $b$ per unit charge along the filament

$$
b:=-e / \lambda
$$

we obtain the condition for counterion condensation:

$$
\xi_{\text {crit }}=1 / z
$$

This relation is interpreted as follows: When the charge density of the polyelectrolyte - represented by $\xi$ - is high enough, counterions will condense onto it and will thereby neutralize charges until its effective $\xi_{\text {eff }}$ equals $\xi_{\text {crit }}$. Otherwise the charge of the polyelectrolyte is too low and no condensation will occur.

The fraction $\Theta$ of the charges of the polyelectrolytes which are compensated by the bound counterions to obtain an effective $\xi_{\text {eff }}=1 / z$ is [83]:

$$
\Theta= \begin{cases}1-\frac{1}{z \xi} & \text { if } \xi>1 / z \\ 0 & \text { if } \xi<1 / z\end{cases}
$$

For important biopolymer systems that were investigated using the concept of counterion theory we compare these important quantities in Tab. 2.3. Note that

\begin{tabular}{llllll}
\hline biopolymer & $\xi$ & $\Theta_{z=1}$ & $\Theta_{z=2}$ & $\Theta_{z=3}$ & $\Theta_{z=4}$ \\
\hline DNA [141] & 4.2 & 0.76 & 0.88 & 0.92 & 0.94 \\
F-actin [131] & 2.8 & 0.64 & 0.82 & 0.88 & 0.91 \\
microtubles [98] & 20.9 & 0.95 & 0.98 & 0.98 & 0.99 \\
\hline
\end{tabular}

Table 2.3: Charge neutralization in the Manning theory. Fraction $\Theta_{z}$ of compensated polyelectrolyte charges for different biopolymers and counterions of valency $z$ studied in aqueous solution at room temperature. 
the table gives values for an aqueous solution. In other solvents, the Bjerrum length (Eq. 2.5) is different to water and therefore the fraction of condensed charges changes. For example, this is relevant for DNA. [22]

The Manning theory predicts which fraction $\Theta$ of the charge of the polyelectrolyte is compensated by bound ions. In this theory the polyelectrolyte is considered as a cylinder. For other geometries like a charged planar surface, the electrostatic energy of a counterion increases proportional to the surface-counterion distance $r$, instead of logarithmically as in cylindrical geometry (Eq. 2.10). As a result, electrostatic attraction outperforms the entropic influence and the surface charges are compensated completely by bound counterions. [90] The question whether a polyelectrolyte is to be considered as a cylinder or planar, depends on the length scales that are important for the system. As we discuss in the following section, cylinders with a radius much bigger than the Debye length can be approximated as planar surfaces. This is due to the fact that the relevant counterions (within one Debye length distance) 'see' the cylinder as planar.

\subsubsection{Ion competition}

When more than one counterion species is in the neighborhood of a polyelectrolyte, there is a competition between the ions to condense onto the polyelectrolyte. We follow the concept of Rouzina and Bloomfield who investigated this competition on the basis of the non-linear Poisson-Boltzmann equation. [105,107]. The concept considers the non-specific electrostatic binding of ions to the polyelectrolyte. In general, there can be also 'specific' binding of ions to biopolymers, where the ions have an affinity to bind at a preferred position at the polyelectrolyte. For DNA this phenomenon is found for magnesium. [3] The polyelectrolyte is modeled as a cylinder with radius $R$ that carries elementary charges $e$ with a charge spacing $b$ (Fig. 2.6a). This simplification implies that all molecular details of the polyelectrolyte are reduced to two quantities. This way the surface charge density $\sigma$ of the cylinder reads

$$
\sigma=\frac{e}{2 \pi b R}
$$

The surface charges of the polyelectrolyte attracts counterions to a final counterion surface concentration of $n_{\mathrm{s}}$. When the radius $R$ of the cylinder is greater than the Debye length $l_{\mathrm{D}}$, the surface of the polyelectrolyte appears to the counterions 
as planar. In this case, it is shown that the relation between $n_{\mathrm{s}}$ and the surface charge $\sigma$ is [49]:

$$
n_{\mathrm{s}}=2 \pi l_{\mathrm{B}}\left(\frac{\sigma}{e}\right)^{2}
$$

Notably, $n_{\mathrm{s}}$ is independent of the valency or the bulk concentrations of the counterions and it is mainly determined by the surface charge of the polyelectrolyte as long as $R>l_{\mathrm{D}}$ holds (constant temperature and surrounding buffer assumed). For DNA this value counts $6.65 \mathrm{M}$, which is much higher than the bulk counterion concentration of the solution. [105] In the presence of one cationic counterion of valency $z_{1}$ its surface concentration $n_{\mathrm{s} 1}$ takes the value $n_{\mathrm{s}}$ (Fig. 2.6b). In the presence of a second cationic counterion of valency $z_{2}$ (surface concentration $n_{\mathrm{s} 2}$ ) the two counterion types compete to populate the polyelectrolyte.

In the case of a high surface charge of the polyelectrolyte ${ }^{1}$, the concentration of the ions at the surface is mainly determined by the electrostatic surface potential $\Psi_{s}$. [105] In thermal equilibrium the surface charge densities $n_{\mathrm{s} i}$ follow the Boltzmann law at the surface of the polyelectrolyte [107]

$$
n_{\mathrm{s} i}=n_{\mathrm{b} i} e^{-z_{i} e \Psi_{s} / k_{\mathrm{B}} T},
$$

\footnotetext{
${ }^{1}$ This is the case for our experiments with vimentin, see Sec. 8.1
}

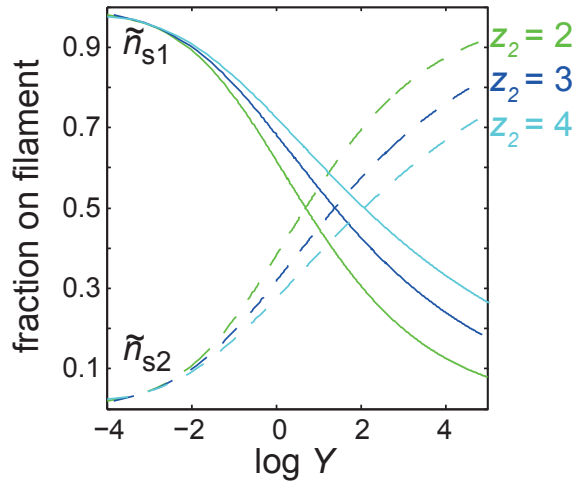

Figure 2.7: Ion competition on the surface of the polyelectrolyte. The fraction $\tilde{n}_{\mathrm{s} 1}$ of the condensed ion population of a monovalent ion $z_{1}=1$ during competitive binding with a multivalent ion with $z_{2}=2,3,4$. (Calculated as in [107]) 
where $n_{\mathrm{b} i}$ is the bulk concentration of the $i$ th ion. When two counterion species $i=1,2$ are considered, the surface potential in Eq. 2.18 is eliminated:

$$
\left(\frac{n_{\mathrm{s} 1}}{n_{\mathrm{b} 1}}\right)^{z_{2} / z_{1}}=\frac{n_{\mathrm{s} 2}}{n_{\mathrm{b} 2}} .
$$

The sum of the surface concentrations of the competing ions are equal to Eq. 2.17: $n_{\mathrm{s}}=n_{\mathrm{s} 1}+n_{\mathrm{s} 2}$. For the derivation of this Eq. we refer to references $[105,107]$, where the non-linear Poisson-Boltzmann equation is investigated in the case of a highly charged surface of the polyelectrolyte. Taken this 'boundary condition' and Eq. 2.19, the direct calculation yields

$$
\frac{\tilde{n}_{\mathrm{s} 2}}{\tilde{n}_{\mathrm{s} 1}^{z_{2} / z_{1}}}=n_{\mathrm{s}}^{z_{2} / z_{1}-1} \frac{n_{\mathrm{b} 2}}{n_{\mathrm{b} 1}^{z_{2} / z_{1}}}=: Y
$$

where the fractional surface concentration $\tilde{n}_{\mathrm{s} 2}:=n_{\mathrm{s} 2} / n_{\mathrm{s}}$ and $\tilde{n}_{\mathrm{s} 1}:=1-\tilde{n}_{\mathrm{s} 2}$ are introduced. $Y$ is a parameter that considers the bulk concentrations and the system variable $n_{\mathrm{s}}$ only. Using these equations the fraction of condensed ions can be calculated as given for the case of a monovalent ion in competition to one multivalent ion type (Fig. 2.7). When we set the monovalent bulk concentration $n_{\mathrm{b} 1}$ to a certain value and increase $n_{\mathrm{b} 2}$, then $Y$ will grow. It is expected that the share of the multivalent component increases, too. This is represented in the course of the dashed lines in Fig. 2.7.

Andresen et al. measured the distribution of mono- and divalent ions around DNA. [4] Their findings are generally in agreement with an investigation of the competitive ion binding that is based on the Poisson-Boltzmann equation. The latter theory is also extendable with regard to the number of competitive counterions as well as for the consideration of counterion radii. [107]

\subsubsection{Attraction mechanisms}

Although there are many experiments that prove the ability of metal ions to induce attraction between polyelectrolytes (Sec. 2.1.3 and [145]), there is no consensus about the precise mechanism. $[6,47,91]$ We review one published idea that is relevant to our system.

The counterions that condense on a polyelectrolyte can basically move freely along its polymeric chain and can be seen as an ideal gas. As the condensed 
counterions are mobile there are thermal fluctuations in their density. When two polyelectrolytes approach each other the charge fluctuations can locally couple leading to a Van-der-Waals-like attraction mechanism. [13] This concept gives the origin of like-charge attraction of polyelectrolytes. However, it remains unclear why monovalent ions do not have the ability to mediate attraction, whereas multivalent ions do (compare Sec. 2.1.3). Within the Poisson-Boltzmann theory, attraction between two polyelectrolytes cannot be explained for any valency of counterions, since it predicts that like-charged objects should always repel each other. An explanation is given in the different capabilities of ions to correlate. $[47,90]$ For this theory we follow the work of Naji et al. [90] Consider positively charged ions of valency $z$ in front of a planar surface that has a surface charge density $\sigma$. The Gouy-Chapman length $\mu$ gives the distance between sur-

a)

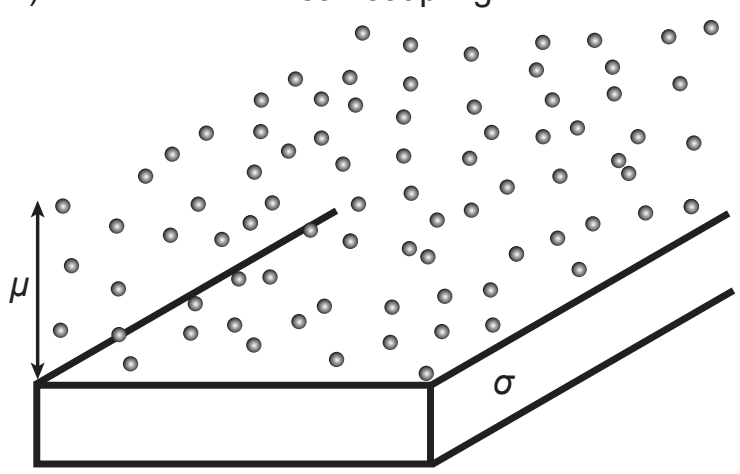

c)

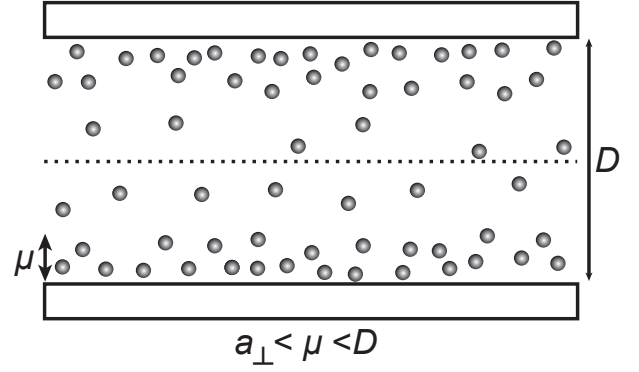

b) strong coupling

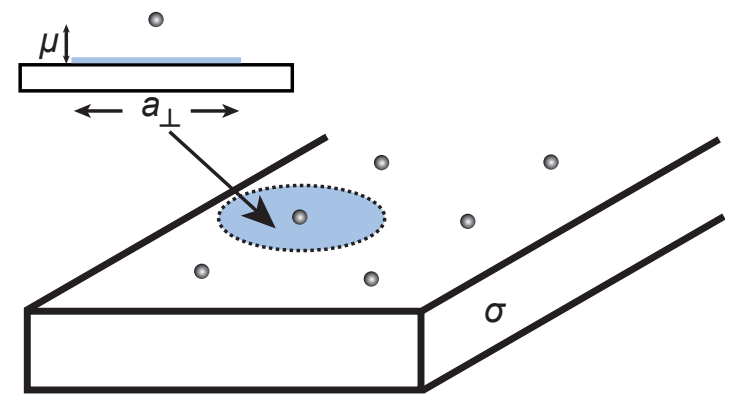

d)

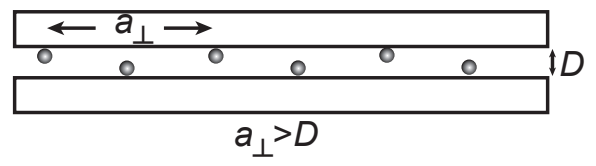

Figure 2.8: Ions in the vicinity of charged planes. a) and b): Ion species of valency $z$ in the vicinity of a surface with charge density $\sigma$. a) In the weak-coupling regime $(\Xi \ll 1)$, the ions form a diffusive 3D-layer, whereas the ions form a $2 \mathrm{D}$ array when they couple in the case of a strong coupling regime $(\Xi \gg 1)$ (b). Due to repulsion, each ion 'sits' in a correlation hole with an approximate size $a_{\perp}$. c) and d): Ions between two surfaces separated by distance $D$. c) For weak coupling of the ions, the osmotic pressure leads to a repulsion of the surfaces. d) However, in the case of strong coupling, $D$ is much smaller than $a_{\perp}$ and attraction between the walls can emerge as a result of attraction between the particles and the plate. (Sketch from [90] with permission of Elsevier) 
face and an ion, at which the surface-ion attraction energy equals thermal energy (represented by the Bjerrum length):

$$
\mu=\frac{e}{2 \pi z l_{\mathrm{B}} \sigma} .
$$

The electrostatic interaction of two elementary charges goes into the definition of the Bjerrum length (Eq. 2.5). The interaction between like-charges of valency $z$ can be compared to thermal energy in a rescaled Bjerrum length

$$
l_{\mathrm{B}, z z}:=\frac{z^{2} e^{2}}{4 \pi \epsilon_{0} \epsilon k_{\mathrm{B}} T}=z^{2} l_{\mathrm{B}}
$$

The electrostatic coupling parameter $\Xi$ is defined as

$$
\Xi:=\frac{l_{\mathrm{B}, z z}}{\mu}=\frac{z^{2} l_{\mathrm{B}}}{\mu} .
$$

It therefore compares the repulsive interaction energy between the ions among themselves to the interaction energy with the surface. Notably, it depends on the square of the ion valency. Two regimes are given as limiting cases: the regime of weak coupling, where $\Xi \ll 1$ and the regime of strong coupling $\Xi \gg 1$. The different coupling strengths lead to a different arrangement of the ions in front of the surface. For weak coupling (Fig. 2.8a) the ions arrange in a 3D manner. In contrast, they arrange into a 2D manner as the ions repel each other in the strong coupling regime (Fig. 2.8b). Each ion has its own 'hole' in which its neighboring ions are depleted. Within this configuration the ion-surface distance $(\approx \mu)$ is much smaller than the spacing $a_{\perp}$ between the ions. Considering the $z$-valent ions between two planar surfaces (Fig. $2.8 \mathrm{c}$ and d) we have a situation that is relevant for polyelectrolytes of comparatively large radius as we find in our experiments (Sec. 8). It is interesting to look at the osmotic pressure that emerges between the walls. For weak coupling it is always repulsive, for strong coupling it is the more attractive the higher the coupling parameter $\Xi$ (see [90] for the quantitative details).

In summary, the attraction mechanism can be explained by correlations between the counterions that condense onto the macro-ions. We describe the two limiting cases of weak and strong coupling. In a real system the coupling strength is in between the two cases, but nevertheless the concept is the same. Naji et al. consider the case of a coupling parameter in the range of $10<\Xi<100$, where they 
also find an onset of attraction.

The theories described above provide explanations for experiments in which attraction of polyelectrolytes is observed. However, there are also other factors that can make the interaction of polyelectrolytes and counterions more complex: These can be for instance hydration and size of counterions or polyelectrolytes. A biopolymer will only follow the assumed geometry in the models in approximation. Depletion forces and entropic forces may also play a role. $[59,145]$ Nevertheless, the theoretical concepts discussed here are important to understand the experiments we present later on.

\subsection{Microfluidic techniques}

Microfluidic methods are used as tools for applications in biophysics. $[17,137]$ In this section, we first present the fundamental physics that make microfluidics a useful tool and we will afterwards cover more detailed findings that are important for discussions later on.

\subsubsection{Flow on small scales}

The Navier-Stokes equation is the differential equation that determines the velocity field $\vec{v}(\vec{r}, t)$ of an incompressible, Newtonian fluid that flows through a channel [29]:

$$
\rho\left(\frac{\partial \vec{v}(\vec{r}, t)}{\partial t}+(\vec{v}(\vec{r}, t) \nabla) \vec{v}(\vec{r}, t)\right)=-\nabla P+\eta \Delta \vec{v}(\vec{r}, t)+f_{\text {ext }}
$$

where $\rho$ is the fluid density, $\eta$ the viscosity, $P$ pressure and $f_{\text {ext }}$ a term for other external forces (per volume). To interpret this equation - particularly with regard to microfluidics - a renormalization to characteristic velocity $U$, time $\tau$, length scale $L$ is useful. This way the Navier-Stokes equation gathers a dimensionless form [29]:

$$
\operatorname{Re}\left(\frac{\partial \tilde{\vec{v}}}{\partial \tilde{t}}+\tilde{\vec{v}} \tilde{\nabla} \tilde{\vec{v}}\right)=-\tilde{\nabla} \tilde{P}+\tilde{\Delta} \tilde{\vec{v}}
$$

where any external forces are neglected for simplicity and the ${ }^{\sim}$-quantities indicate the renormalized values. Additionally, the Reynolds number Re is introduced 
as dimensionless quantity, which compares inertial to viscous forces:

$$
R e:=\frac{\rho L U}{\eta}
$$

For microfluidics we typically have aqueous solutions at room temperature, the length scales are in the order of $\mu \mathrm{m}$ and the velocities in the order of $\mathrm{mm} / \mathrm{s}$. Therefore, a typical order of $R e$ for microfluidics is $R e \ll 1$ which is referred to as low Reynolds number. The implications of a low Re for bacterial swimming have been vividly demonstrated by Purcell. [99] As a consequence of a $R e \ll 1$ the left hand side in Eq. 2.25 - which represents inertial forces - becomes generally irrelevant for microfluidics and the flow velocity is governed by the balance between pressure (left side) and friction (right side):

$$
\nabla P=\eta \Delta \vec{v}
$$

This equation is known as the Stokes equation and it indicates that in a typical microflow experiment inertia and time play no role and therefore any turbulence is suppressed by friction. Instead, we have a purely laminar flow. [99] The laminarity of the flow is what makes microfluidics a precise tool. Note that there are exceptions to this rule and there are special cases in which inertia plays a role in microflow. [37]

\subsubsection{Droplets as microcontainers}

When two immiscible fluids like oil and an aqueous phase get in contact, one of them can disperse to microfluidic droplets. This production of droplets can take place in a defined way in a flow focusing geometry in which the dispersed phase is focused by the continuous phase. [7] The continuous phase exerts shear on the dispersed phase, which pinches off. [112] To avoid coalescence of droplets a surfactant is added to the continuous phase. [11]

Droplets are used as microcontainers as they have a typical diameter in the order of 1-100 $\mu \mathrm{m}$. [7,114] They have been successfully used in different fields of research, e.g. for cell encapsulation $[28,67,68,109,111]$ and high-throughput screening enzymatic activity [12], protein networks [42], directed evolution [43] and X-ray studies on liquid crystals [147] to mention a few. The wide spreading of droplets may well be due to their striking advantages like small sample con- 
a) dropspots

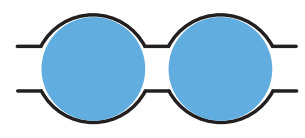

top view
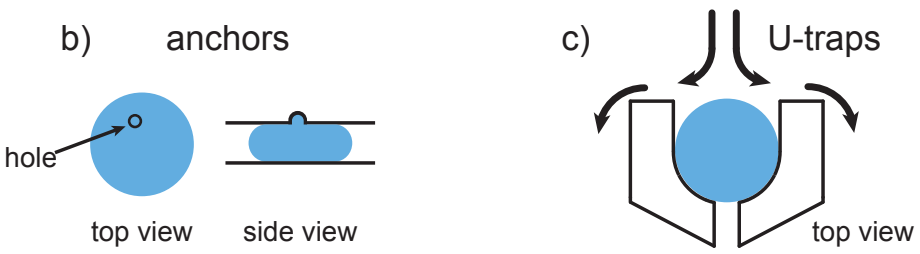

Figure 2.9: Drop trapping concepts. Microfluidic methods for drop trapping via a) constrictions [109], b) small holes in the channel walls [1] and c) U-shaped traps [58].

sumption of precious samples (generally a feature of microfluidics), their large number (high-throughput) [12,43], their small volumes so that chemicals become rapidly detectable, i.e. metabolic products of cells, or the very small confinement they provide. [67] Although the permeability of oil phases and devices are often exploited during cell culturing in drops [67], droplets are more commonly considered as isolated containers. However, it is known that there can be exchange of chemicals between drops which depends on the oil, the surfactant and the aqueous phase of the droplet emulsion. [10,116]

Droplet-based experiments benefit from the various techniques available to manipulate droplets like drop fusion or splitting, sorting or other types of manipulation [112]. For our purposes, methods for drop trapping are of particular interest. We discuss three important concepts to trap drops: An array of constrictions along the channel wall leads to a trap mechanism when the flow in the channel is stopped (Fig. 2.9a). [109] Another method bases on holes in the channel 'roof' [1]. Both techniques profit from surface energy minimization leading to drop trapping. A third method drives the drops into a U-shaped trap by flow. [58] In all these techniques the drops can be released again by changing flow rates appropriately. Additionally, there exist further drop trapping principles [115] and also modifications of the illustrated examples [10,96,126, 127]. An important issue during trapping of water drops in oil as carrier fluid can be drop shrinkage since one of the common microfluidic device materials PDMS (polydimethylsiloxane) is permeable to water. $[58,61,109,113]$. Ways prevent or decrease this effect are, for example, switching to water impermeable device materials [14], channel coating rendering the channels waterproof [20] or water saturation of the PDMS device [109]. 


\subsubsection{Diffusion and mixing}

Turbulence is an important mechanism to mix in the macroscopic world, mixing coffee and milk is one example. However, since the Stokes equation (2.27) dictates that microflow will be laminar, this mechanism is not applicable in microfluidics. Instead, mixing is based on diffusion processes. Molecules with a diffusion constant $D$ will diffuse in a three dimensional space over a distance $L$ within a time $t$. $L$ is approximated by the second moment of position given for an ensemble of these molecules:

$$
L^{2} \approx\left\langle\vec{x}^{2}\right\rangle=6 D t
$$

Fast mixing is often needed in microfluidics. Convection flows can be used to accelerate mixing times. Because the diffusion process itself cannot be accelerated, the length scales that particles travel by diffusion have to become shorter to accelerate mixing. This principle has been realized impressively by the staggered herringbone mixer, which uses channel wall structures to force chaotic patterns onto the fluids which shortens diffusion paths. [125]

Another effect in which convection accelerates mixing is Taylor dispersion. [119, 133] The mechanism is best illustrated for of a plug-like fluid segment of test particles with a diffusion constant $D$ in Poisson-flow. (Fig. 2.10, with description). Taylor dispersion will blur the plug-like concentration profile as the fluid flows. The strength of this process is expressed by the dimensionless Péclet number $P e$ which connects the characteristic diffusion time with the axial convection time [29]:

$$
P e=\frac{\text { diffusion time }}{\text { convection time }}=\frac{U a}{D},
$$
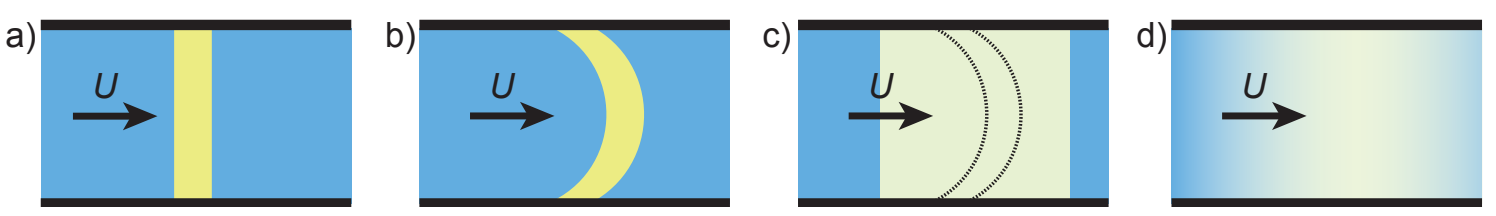

Figure 2.10: Taylor dispersion. a) A segment of test-particles (yellow) moves forward in a laminar flow. b) If there was no diffusion, the segment would be stretched into a parabolic-like form. c) However, radial diffusion superimposes this process and blurs the profile in all directions. d) Continuing the overlay of these two processes leads to a particle concentration profile that is Gaussian-like in flow direction. (Based on [119]) 
a)

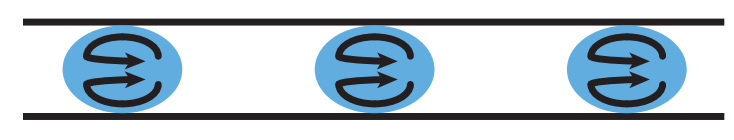

b)

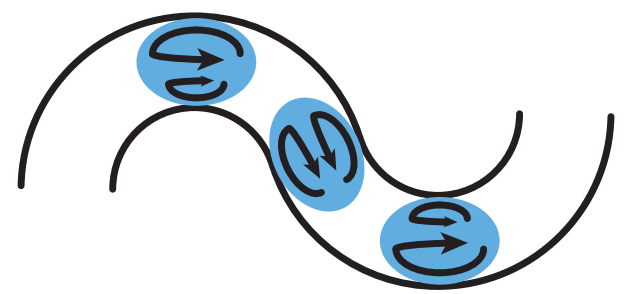

Figure 2.11: Flows inside of drops during drop movement. During the movement of plug-like drops, internal vortices emerge which are a) stationary in the drop for a straight channel and b) periodically changing over time in a serpentine channel. (See e.g. [27] for review)

with the characteristic length $a$ and the axial velocity $U$. Using $P e$, the effective axial diffusion constant in a cylindrical channel can be approximated [29]:

$$
D_{\mathrm{eff}} \approx\left(1+\frac{P e^{2}}{72}\right) D
$$

For other channel geometries this relation would differ. As $P e$ is often large in microfluidics (see below), mixing by Taylor dispersion is very effective $\left(D_{\text {eff }} \gg D\right)$. For microfluidic droplets there is also a mechanism that accelerates mixing and we follow Bringer et al. for its description. [27] This phenomenon occurs in moving drops that are plug-like, which means that they have channel-wall contact during movement (Fig. 2.11). There are basically two situations to compare: plug-like drops moving through a straight channel and plug-like drops moving to a serpentine channel. In the first case, the drop-wall contact of the drop induces two (stationary) vortices into the drop internals (Fig. 2.11a). Due to this circulation in the drop the striation length $s$ (length to diffuse) is reduced continuously when the drop moves. After traveling the distance $d$ the striation length is given by

$$
s(d)=s(0) \frac{l}{d}
$$

where $s(0)$ is the initial striation length and $l$ the drop length. This can be a quite effective process: For example, when a drop has traveled only ten times its own length, the time for mixing $t_{\text {mix }}$ can be approximated with Eq. 2.28 to $\approx 0.01 t_{\text {diff }}$ compared to the case of the immobile drop where mixing happens via diffusion only in the time $t_{\text {diff. }}$ Mixing becomes even more effective, when the drops pass a serpentine channel which induces instationary internal vortices in the drops (Fig. 
2.11b). In this case a chaotic pattern emerges in the drop internals and the ratio of $t_{\text {mix }}$ and $t_{\text {diff }}$ can be compared via the Péclet number:

$$
\frac{t_{\text {diff }}}{t_{\text {mix }}}=\frac{P e}{m \times \log P e},
$$

where $m$ denotes the plug length in units of the channel width. As $P e$ numbers are often large (typical: $10<P e<10^{5}$ [124]), the mixing time for the drops moving through serpentines will easily outperform pure diffusive mixing, i.e. $t_{\text {diff }} \gg t_{\text {mix }}$. 



\section{3}

Materials and methods

In this chapter, we will discuss the applied materials and methods that are used for the experiments. Although many of the used methods are well established in the microfluidic or protein research field, there are some steps that are special and indispensable to reproduce the experiments. [34]

\subsection{Preparation of vimentin protein}

\subsubsection{Vimentin mutants}

The one-letter ${ }^{1}$ representation of the human vimentin (hVim) wild-type amino acid sequence is provided by the European Nucleotide Archive database (identification: Z19554). [8] The head and tail domain flanking the rod domain are indicated. The segmentation of the sequence into head, rod and tail is made as in reference [52]. There are also other publications that provide another segmentation (e.g. [32]).

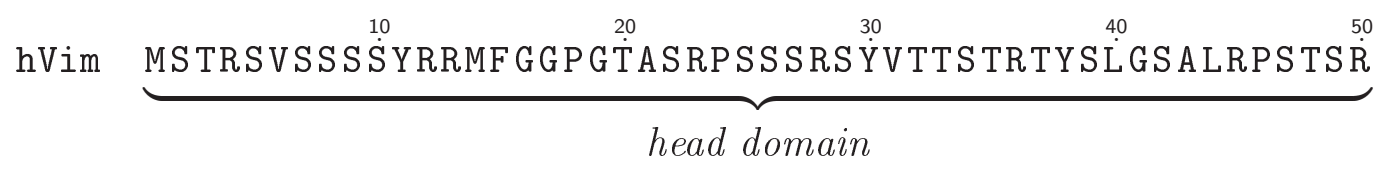

\footnotetext{
${ }^{1} \mathrm{~A}=$ alanine, $\mathrm{C}=$ cysteine, $\mathrm{D}=$ aspartate (aspartic acid), $\mathrm{E}=$ glutamate (glutamic acid), $\mathrm{F}=$ phenylalanine, $\mathrm{G}=$ glycine, $\mathrm{H}=$ histidine, $\mathrm{I}=$ isoleucine, $\mathrm{K}=$ lysine, $\mathrm{L}=$ leucine, $\mathrm{M}=$ methionine, $\mathrm{N}=$ asparagine, $\mathrm{P}=$ proline, $\mathrm{Q}=$ glutamine, $\mathrm{R}=$ arginine, $\mathrm{S}=$ serine, $\mathrm{T}=$ threonine, $\mathrm{V}=$ valine, $\mathrm{W}=$ tryptophan, $\mathrm{Y}=$ tyrosine. (From: [78])
} 


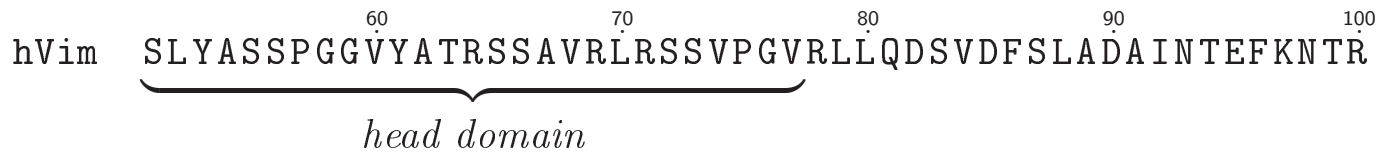

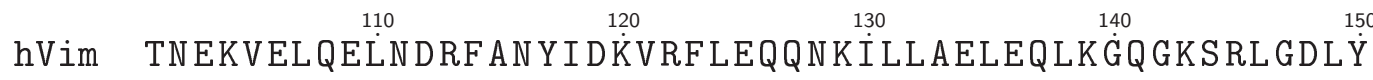

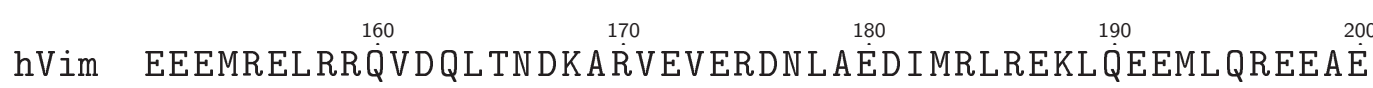

hVim NTLQSFRQDV்DASLARLDLERKVESLQEEI AFLKKLHEEEIQELQAQIQ $\stackrel{240}{240}$

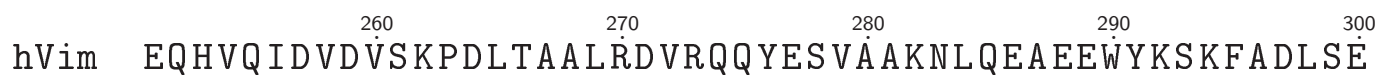

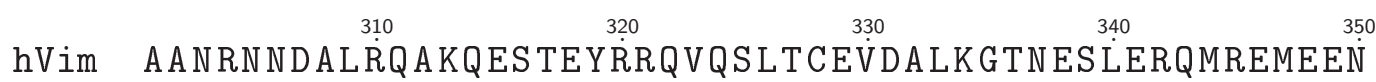
$\uparrow$ cysteine

hVim FAVEAANYQDTIGRLQDEIQNMMEEMARHLREYQDLLNVKMALDIEIATY்

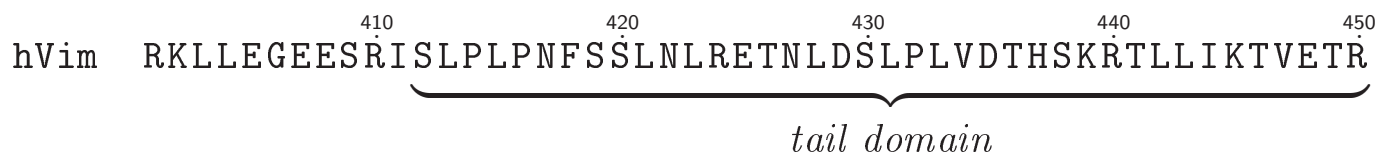

hVim $\underbrace{\text { DGQVINETSQ̈HHDDLE }}_{\text {tail domain }}$

We use a human vimentin plasmid that is modified compared to its wild-type amino acid sequence and kindly provided by the group of Harald Herrmann (German Cancer Research Center (DKFZ), Heidelberg, Germany). The resulting vimentin mutant has two point modifications compared to the wild-type protein: The cysteine at position 328 is replaced by an alanine and there is an additional cysteine at the C-terminus of the protein. The repositioning of the cysteine to 
the end of the flexible tail allows for the attachment of a fluorescent label that does not disturb lateral annealing of vimentin filaments. [50,104] These are minor changes compared to the wild-type human vimentin and they do not change the net charge of vimentin (Sec. 8.1). For simplicity we refer to the mutant only as 'vimentin' (Vim). This protein is used in most of our experiments and we explicitly mention where this is not the case. For special questions we investigate two other mutants that have a (partly) truncated tail compared to the wild-type: ' $\Delta \mathrm{C} 455$ ' and ' $\Delta \mathrm{C} 411$ '. For these three mutants the one-letter code are given at the positions, where changes compared to the wild-type occur:

vimentin:

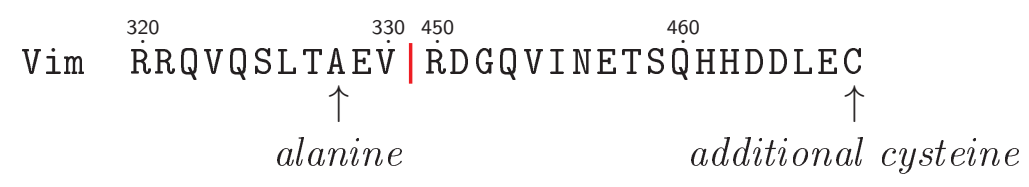

$\Delta$ C455 vimentin $(\Delta \mathrm{C} 455 \mathrm{~V}$ im $)$ misses the last 11 amino acids compared to $\mathrm{hVim}$ [76]:

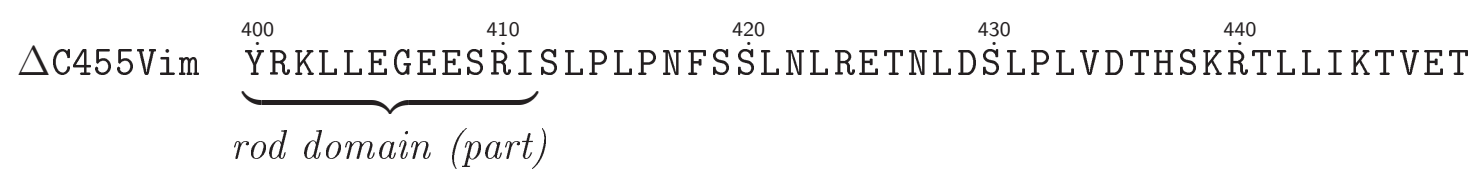

$\triangle$ C455Vim $\stackrel{450}{\mathrm{R} D G Q V I}$

$\Delta \mathrm{C} 411$ vimentin $(\Delta \mathrm{C} 411 \mathrm{Vim})$ misses the last 55 amino acids compared to $\mathrm{hVim}$ [76]:

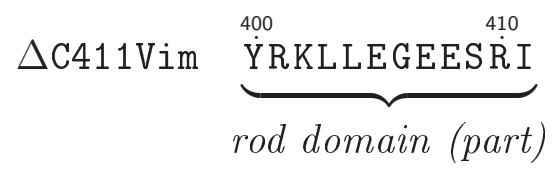




\subsubsection{Production and labeling}

Escherichia coli is transformed with the plasmid for vimentin and the expressed protein is purified from inclusion bodies following the protocol of Herrmann et al. [53]. The protein is then stored in a storage buffer consisting of $8 \mathrm{M}$ urea, $5 \mathrm{mM}$ tris(hydroxymethyl)aminomethane (TRIS) at $\mathrm{pH} 7.5,1 \mathrm{mM}$ ethylenediaminetetraacetic acid (EDTA), $0.1 \mathrm{mM}$ ethylene glycol tetraacetic acid (EGTA), $1 \mathrm{mM}$ DTT (dithiothreitol) and $10 \mathrm{mM}$ methyl ammonium chloride at $-80^{\circ} \mathrm{C}$.

We fluorescently label our protein similarly to the description in [143]. For this purpose, long-time stored vimentin is dialyzed first against $4 \mathrm{M}$ urea, $12 \mathrm{mM}$ phosphate buffer ( $\mathrm{PB}, \mathrm{pH}=7.5$ ) for $0.5 \mathrm{~h}$, against $20 \mathrm{mM} \mathrm{PB}$ for first $0.5 \mathrm{~h}$ and then another time for $1 \mathrm{~h}$. For this step a dialysis tubing with a molecular weight cut-off (MWCO) of $50 \mathrm{kDa}$ is used (Spectra/Por 7, Spectrum Europe B.V., Breda, Netherlands). $150 \mu \mathrm{g}$ Alexa Fluor ${ }^{\circledR} 488$ dye (C5 maleimide, Invitrogen $\mathrm{GmbH}$, Darmstadt) dissolved in $20 \mu \mathrm{L}$ dimethyl sulfoxide (purity $\geq 99.8 \%$ sterile filtered, Carl Roth $\mathrm{GmbH}+$ Co. KG, Karlsruhe, Germany) is added to $900 \mu \mathrm{L}$ of protein $(\mathrm{c}=1 \mathrm{mg} / \mathrm{mL})$. After light protected incubation overnight, $100 \mu \mathrm{L}$ of $1 \mathrm{M} \mathrm{L}$ cystein is added to bind unspecifically bound label. The labeled protein is finally eluted in MidiTrap ${ }^{\mathrm{TM}}$ columns (PD MidiTrap ${ }^{\mathrm{TM}}$ G-25, GE Healthcare, München, Germany) following the manufacturers protocol with the following elution buffer: $9 \mathrm{M}$ urea,10 $\mathrm{mM}$ phenylmethylsulfonylfluoride ( $\geq 99 \%$, Serva Electrophoresis $\mathrm{GmbH}$, Heidelberg, Germany), $10 \mathrm{mM}$ DTT and $10 \mathrm{mM}$ TRIS (Carl Roth GmbH + Co. KG, Karlsruhe, Germany, $\mathrm{pH}=7.5)$. The elution process is done twice to optimize the labeling results. This way the free label is removed and the protein is transferred into a storage buffer. The protein is then stored at $-80^{\circ} \mathrm{C}$. The labeling efficiency is high and measurements suggest that a fluorescent dye is attached to each vimentin monomer during the labeling process.

The tail truncated vimentin mutants are labeled analogously using ATTO $647 \mathrm{~N}$ dye (N-Maleimide, ATTO-TEC GmbH, Siegen, Germany) and dialysis tubing of MWCO 25 kDa (Spectra/Por 7, Spectrum Europe B.V., Breda, Netherlands). 


\subsubsection{Dialysis and assembly}

The protein concentration is diluted during the labeling process. As we mix unlabeled protein of higher concentration with labeled protein, the overall protein concentration increases and the assembly results are optimized. Prior to the usage in the experiments the protein has to be reconstituted from storage buffer. Additionally, we tune the vimentin concentration to the desired value with this method. Mixing of the protein prior to dialysis is also important, since tetramers carrying both unlabeled and labeled monomers are formed only then. We use two assembly protocols which lead to an average filament length of about $10 \mu \mathrm{m}$ (protocol A) or $5 \mu \mathrm{m}$ (protocol B). Additionally, the protein concentration is smaller with protocol B. The protocol B is the preferred one, since the protein solution with smaller filament lengths and at lower protein concentration is more easily encapsulated in microfluidic drops (further discussed in Sec. 5.3 and 9.1.5).

Assembly to filaments: protocol A

(for experiments with device C; Sec. 4.1 and 7.1)

Dialysis steps: $1 \mathrm{~h}$ against $6 \mathrm{M}$ urea, $0.8 \mathrm{mM} \mathrm{PB} \mathrm{pH}=7.5,1 \mathrm{~h}$ against $2 \mathrm{mM}$ PB and again over night against $2 \mathrm{mM}$ PB. The total protein concentration after dialysis is c $=1.2 \mathrm{mg} / \mathrm{mL}$ ( $40 \%$ labeled). The dialyzed protein is assembled for $15 \mathrm{~h}$ in an epi cup at the following assembly conditions: $\mathrm{c}=0.6 \mathrm{mg} / \mathrm{mL}, 100 \mathrm{mM} \mathrm{KCl}$, $2 \mathrm{mM} \mathrm{PB}, \mathrm{pH}=7.5,37^{\circ} \mathrm{C}$.

Assembly to filaments: protocol B

(for experiments with device $\mathrm{T}$ and on cover glasses; Sec. 4.2, 7.2 and 7.4)

Dialysis steps: $1 \mathrm{~h}$ against $6 \mathrm{M}$ urea, $0.8 \mathrm{mM}$ PB $\mathrm{pH}=7.5$, two times against $2 \mathrm{mM} \mathrm{PB}$ for a total of $3 \mathrm{~h}$. The total protein concentration after dialysis is $c=0.44 \mathrm{mg} / \mathrm{mL}$. This protocol is also used for the tail-truncated mutants. For $\Delta$ C411 vimentin we obtain a total concentration of $\approx 0.3 \mathrm{mg} / \mathrm{mL}$ and for $\Delta C 455$ vimentin $\approx 0.1 \mathrm{mg} / \mathrm{mL}$. The dialyzed protein is assembled for $6 \mathrm{~h}$ in a small cup at the following assembly conditions: $c=0.22 \mathrm{mg} / \mathrm{mL}$ (20\% labeled), $100 \mathrm{mM}$ $\mathrm{KCl}, 2 \mathrm{mM} \mathrm{PB}, \mathrm{pH}=7.5,37^{\circ} \mathrm{C}$. The mutants are assembled using the same protocol $\left(c_{\Delta \mathrm{C} 411}=0.15 \mathrm{mg} / \mathrm{mL}\right.$ (all labeled), $c_{\Delta C 455}=0.05 \mathrm{mg} / \mathrm{mL}$ (all labeled) $)$. 


\subsection{Fabrication of microfluidic devices}

\subsubsection{Photolithographic methods}

We produce the microfluidic devices following the concept of 'soft lithography'. $[39,101]$ For this purpose, we design our microfluidic channels using AutoCAD software (Autodesk, München, Germany). The resulting photomasks (simplified example in Fig. 3.1a) are printed as emulsion film on polyester (JD PhotoTools, Oldham, United Kingdom and Selba S. A., Versoix, Switzerland) with a sufficiently high resolution for features with a minimum size of about $5 \mu \mathrm{m}$.

The production of a 'master' for the PDMS devices is performed in a cleanroom to avoid dust impurities on the structures and unwanted photoresist exposure to UV-light. A two inch silicon wafer is rinsed with isopropanol. The wafer is

a)

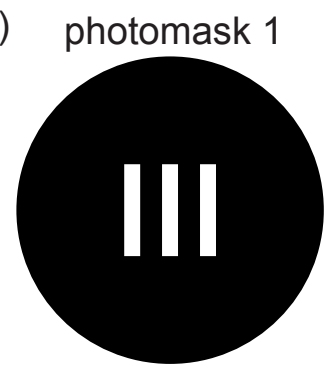

b)

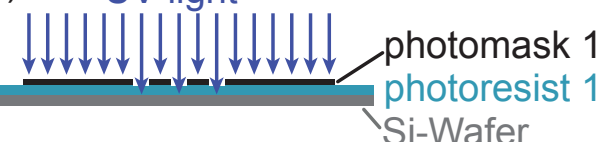

c) UV-light

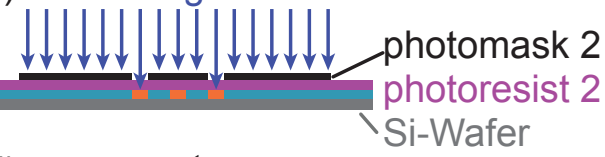

d) master

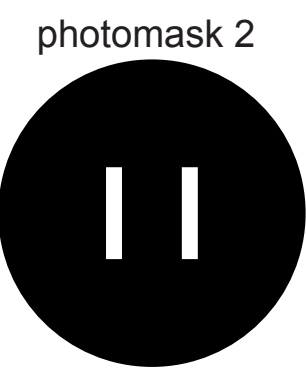

photoresist 1
Si-Wafer

Figure 3.1: Sketch of device production. The microfluidic devices are produced using PDMS-based soft-lithography. a) In this procedure, photomasks (exemplary sketched with a simple channel design) are used to cover photoresist during UV-light exposure (b,c). In doing so, a 'master' of the microfluidic channels is created (d) which is molded with the polymer PDMS (e). After curing in the oven, the hardened, but flexible device is sealed with a cover slide (f). Optional treatment of the devices, like water saturation, can be performed before usage. To minimize the risk of leakage, the tubing connection is sealed with glue $(\mathrm{g})$. The diameter of the wafer is two inches and the channel thickness is scaled up for demonstration purposes in the sketch. 

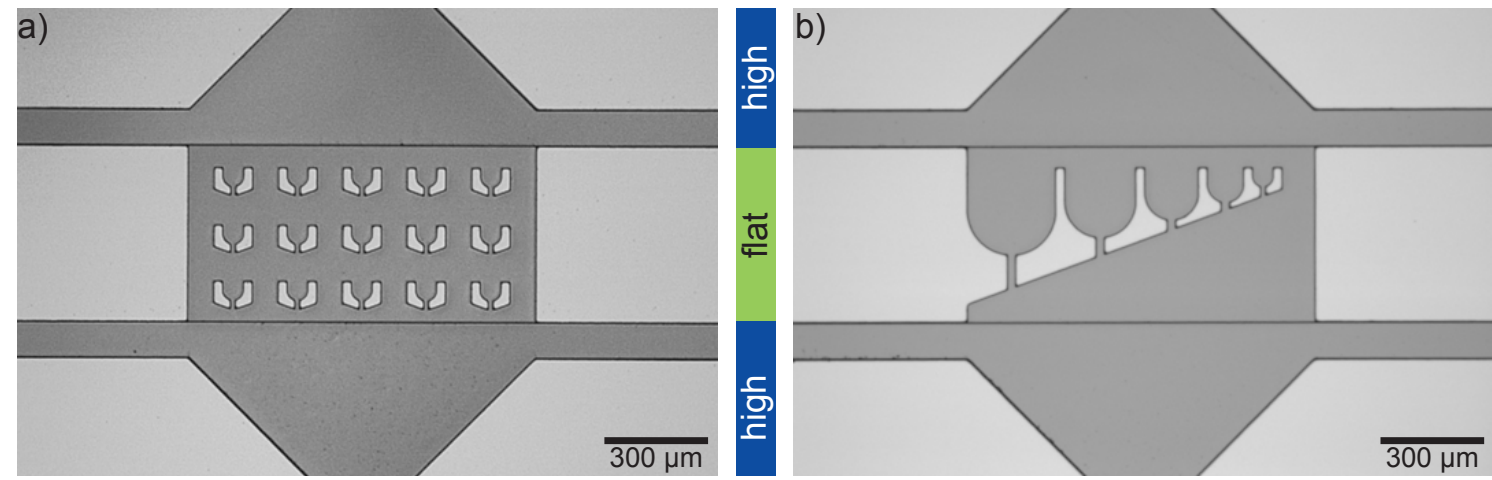

Figure 3.2: Double-layered devices. Device 'master' imaged with reflected lightmicroscopy: Silicon-wafer as substrate for the photoresist that molds the microfluidic channels. These devices contain two layers of resist, which results in a flat and high channel region in the device. This concept is used for droplet manipulation (Sec. 4.2). (Adapted from reference [35] with permission from The Royal Society of Chemistry)

\begin{tabular}{lccccc}
\hline height & resist type & max. spin speed & soft bake & UV-exposure $@ 22 \frac{\mathrm{mW}}{\mathrm{cm}^{2}}$ & PEB \\
\hline$\approx 18 \mu \mathrm{m}$ & SU8-3025 & $5000 \mathrm{rpm}$ & $15 \mathrm{~min}$ & $18 \mathrm{~s}$ & $5 \mathrm{~min}$ \\
$\approx 15 \mu \mathrm{m}$ & SU8-3025 & $10000 \mathrm{rpm}$ & $15 \mathrm{~min}$ & $18 \mathrm{~s}$ & $5 \mathrm{~min}$ \\
\hline
\end{tabular}

Table 3.1: Lithography parameter. Details for the production of the 'master' copy device production. A two layered device is obtained by applying both recipes for one layer in sequence. The given values can only be considered as guidelines, as resists change their viscosity when aging. To be sure about the obtained resist thickness, the resulting layers are measured using a profilometer. $(\mathrm{PEB}=$ post exposure bake)

dried at $200^{\circ} \mathrm{C}$ on a hot plate for 10 minutes so that any isopropanol or water layer evaporates. SU-8 negative photoresist (MicroChem, Newton, USA) is spin coated onto the silicon wafer. After spin coating, the resist is placed on a hot plate at a temperature of $95^{\circ} \mathrm{C}$ in a soft bake step. Using a mask aligner (MJB4, Süss MicroTec AG, Garching, Germany) the photomask is brought into vacuum contact with the coated wafer and exposed with UV-light at a wavelength of $365 \mathrm{~nm}$ in regions where the mask is transparent (Fig. 3.1b). The exposure time is chosen with respect to the resist type and resist height. The exposed resist is baked in a post exposure step for 5 minutes. During this process the exposed regions in the resist are cross-linked. [85]

In cases where only one resist layer is wanted (Sec. 4.1) the wafer is then developed in SU-8 developer (MicroChem), i.e. resist which is not cross-linked is dissolved completely. In cases where another layer is to be deposited on the first layer, the procedure starting from spin coating to the post exposure step is re- 
peated accordingly using the mask for the second layer (Fig. 3.1c). Details of the production process are given in Tab. 3.1. The resulting resist - wafer unit is called device 'master' of the microfluidic channel design (Fig. 3.1d). Its actual channel height is measured after its production using a profilometer 'Dektak 6' (Veeco Instruments Inc., Mannheim, Germany). In Fig. 3.2 we show two wafers of the device design as described in Sec. 4.2. These wafers consist of two layers, which results in a comparatively flat $(18 \mu \mathrm{m})$ and a high region $(18+15 \mu \mathrm{m})$.

\subsubsection{Device production}

One master of the microfluidic device can be used to produce many microfluidic devices. For this purpose, the master is molded with PDMS mixed with crosslinker at a ratio of 10:1 (Silgard 184, Dow Corning GmbH, Wiesbaden, Germany). This mold is baked at $65^{\circ} \mathrm{C}$ in an oven for at least one hour (Fig. 3.1e). The resulting PDMS replicate is peeled off the wafer and holes for tubing connection are punched into the device (diameter $=0.75 \mathrm{~mm}$ ). This device and a cover slide are surface-treated in an air plasma cleaner (Harrick Plasma, Ithaca, USA) and bonded as they are brought into contact afterwards (Fig. 3.1f). The devices are stored in Milli-Q water for 1-2 days and are water saturated. This reduces unwanted drop shrinkage during the experiment. This treatment is used for experiments in which drops are in wall contact for a longer time on the order of hours (Sec. 4.1). For experiments in which the drops have only contact to the walls for some minutes (Sec. 4.2) this treatment is not used since drop shrinkage is so small that its influence is acceptable (Sec. 5.2). Another treatment of the devices is the flushing of the channels with 'Ombrello' (Autoserv, Sinzheim, Germany). Ombrello binds to the channel walls and renders them hydrophobic. This step is performed in each experiment and ensures that the oil phase of the droplet emulsion wets the channel walls. Polyethylene tubing (Intramedic ${ }^{\mathrm{TM}}$ PE20, BD, Franklin Lakes, USA; inner diameter $=380 \mu \mathrm{m})$ is inserted into the punched holes and self-sealed as the outer tubing diameter $(1.09 \mathrm{~mm})$ exceeds the punch diameter. Using the fast curing 'Loctite' glue (two component: Loctite 406 and 770, Henkel, Düsseldorf, Germany) the tubing connection is sealed before the experiment to minimize the risk of leakage (Fig. 3.1g). 


\subsection{Emulsion production in microfluidic channels}

The microfluidic devices are connected to gastight glass syringes (250-2500 $\mu \mathrm{L}$ volume, Hamilton, Bonaduz, Switzerland) via polyethylene tubing. These syringes are driven by precise syringe pumps (neMESYS, Cetoni GmbH, Korbußen, Germany). In a flow focusing geometry (Sec. 4.1 and 4.2 ) the aqueous, protein carrying phase (see Sec. 3.1.3) is focused by an oil phase and thereby the aqueous phase is encapsulated into drops. The oil phase consists of a fluorocarbon oil (Fluorinert $^{\mathrm{TM}}$ FC-40, 3M Deutschland GmbH, Neuss, Germany) to which a surfactant (Raindance Technologies, Lexington, USA) is added at $1.8 \%(\mathrm{w} / \mathrm{w})$. The surfactant stabilizes the emulsion while minimizing interaction of its head groups to the drop content. The surfactant is a biocompatible block-copolymer. [55] The aqueous phases are composed of three different aqueous fluids. The composition of the three aqueous components is in detail (Fig. 4.1 or 4.4 ):

Left channel (aq 1): A multivalent salt (one of the following: $31 \mathrm{mM} \mathrm{MgCl}_{2}$, $18 \mathrm{mM} \mathrm{Co}\left(\mathrm{NH}_{3}\right)_{6} \mathrm{Cl}_{3}$ or $\left.7 \mathrm{mM} \mathrm{Co}\left(\mathrm{NH}_{3}\right)_{6} \mathrm{Cl}_{3}\right), 100 \mathrm{mM} \mathrm{KCl}, 2 \mathrm{mM}$ PB. Central channel (aq 2): $100 \mathrm{mM} \mathrm{KCl,} 2 \mathrm{mM}$ PB. Right channel (aq 3): The protein is supplied in its assembly conditions (Sec. 3.1.3) with the exception that it is at room temperature. As a consequence of this setup the assembly conditions (100 mM $\mathrm{KCl}, 2 \mathrm{mM} \mathrm{PB}, \mathrm{pH}=7.5$ ) remain the same for the protein when it is encapsulated in drops, but only multivalent salt conditions change.

\subsection{Fluorescence microscopy}

For a general review of (fluorescence) microscopy we refer to [75] and give the specifications of the imaging setups we use. Microfluidic devices and time-lapse series of moving drops are imaged with an inverted microscope (IX71 or IX81, Olympus, Hamburg, Germany). These images are taken in brightfield microscopy using typically a $10 \times$ objective. Rapid image sequence recording is needed in cases where drops are moving fast. For these records we use a fast camera (pco. 1200s, PCO AG, Kelheim, Germany).

Fluorescence images are taken with the IX81 microscope. For experiments with device C (Sec. 4.1 and 7.1), we use a $40 \times$ oil-immersion objective (UPlanFLN, Olympus) in epifluorescence microscopy and record the images using an Orca-R2 
camera (Hamamatsu Photonics Deutschland $\mathrm{GmbH}$, Herrsching am Ammersee, Germany). The microfluidic devices are stored in a microscope incubation chamber (INUG2E-ONICS, Tokai Hit CO., Ltd, Gendoji-cho, Fujinomiya-shi Shizuokaken, Japan) during image acquisition to minimize drying of the water-saturated PDMS devices. Air condition is roughly $37^{\circ} \mathrm{C}$ at saturated humidity in the chamber.

For experiments with device T (Sec. 4.2 and 7.2) and cover glasses (Sec. 7.4.2), confocal microscopy with a silicone-oil immersion $60 \times$ objective (UPLSAPO 60XS, Olympus) is used at the IX81 setup (with FV-1000 confocal unit, Olympus). For fluorescence imaging of protein tagged with Alexa Fluor ${ }^{\circledR} 488$, an argon laser $(488 \mathrm{~nm})$ is used as light source, the excitation dichroic mirror is DM405/488/561/ 633 and the emission filter BA505IF. For imaging of protein tagged with a ATTO $647 \mathrm{~N}$ dye, a diode laser is used for exitation $(635 \mathrm{~nm})$ and the emission filter BA575-675 is used. The images in Sec. 7.4.1 are also taken with the same microscope, but in epifluorescence mode (filter set U-MNIBA2 in combination with a xenon burner). In this case, the images are taken with the Orca-R2 camera (all filters: Olympus). 


\section{4 \\ Microfluidic devices for protein studies}

The aggregation of vimentin filaments was studied in drops within microfluidic devices. We tailored the 'device $C^{\prime}$ and the 'device $T^{\prime}$ to answer different questions about the aggregation of the protein in the presence of salt.

\subsection{Device C: Concentration dependence}

In this section, we describe a microfluidic device that was designed to study the dependence of vimentin protein aggregation on salt concentration. In the following, this device is abbreviated as 'device $C^{\prime}$, accounting for its main purpose concentration studies.

\subsubsection{Motivation}

The concept of the device $C$ is as follows: Microfluidic drops are produced and in the aqueous phase of the emulsion, protein filaments and multivalent ions initially get in contact. While the drops are produced, the multivalent ion concentration is changed over time. A series of these differently composed drops is conducted into constrictions and - as the overall flow in the device is stopped - the drops are trapped for hours. The content of a few hundred drops is then imaged. We have described a precursor device in [34].

The main advantage of investigating proteins with this microfluidic tool is the 
encapsulation of the protein into drops, which prevents sticking of the protein to the channel walls. Furthermore, magnesium ion concentrations in a series of produced drops are tuned precisely. This way the microfluidic studies allow direct comparisons between the protein networks at different multivalent ion conditions because all small 'test tubes' are treated the same before the protein is imaged. This underlines the high reliability of the findings. The confinement of the protein in the small drop volume and low sample consumption are also of relevance. A detailed discussion of the meaning of these features is given in Sec. 9.1.6. We have published the function of this tool as well as the results of its application to vimentin in Biomicrofluidics. [36]

\subsubsection{Device design and function}

The device for the production, collection and long-time storage of aqueous microfluidic drops is illustrated in Fig. 4.1. It is equipped with one oil inlet, another oil supply at the bottom that functions either as inlet or as outlet and six outlets (Fig. 4.1). Additionally, there are three aqueous inlets that jointly compose the dispersed phase of the emulsion. [118] Typical flow velocities during the usage of the device are listed in Tab. 4.1. For the three aqueous components, we choose multivalent salt solution on the left side (aq 1) and at the opposite side the protein solution (aq 3) (Fig. 4.1b). In between, there is the inlet that contains only buffer (aq 2). This way, the first contact of multivalent salt and protein solution is retarded (Sec. 5.1). Due to the laminar flow regime, the three solutions flow side-by-side. When the aqueous fluid reaches the cross channel, it is focused by the oil phase and - as a result of the interplay between shear and interfacial forces - drops pinch off (Sec. 2.3.2). [7] A constant flow rate is applied for the protein

\begin{tabular}{cccccc}
\hline procedure & $u_{\text {top oil }}$ & $u_{\text {bottom oil }}$ & $u_{\text {aq 1 }}$ & $u_{\text {aq } 2}$ & $u_{\text {aq } 3}$ \\
\hline startup & 23 & +4 & 4.7 & 4.7 & 4.7 \\
drop collection & 23 & -2 & zig-zag: 0-9.4 & $9.4-u_{\text {aq 1 }}$ & 4.7 \\
drop content imaging & 0 & 0 & 0 & 0 & 0 \\
\hline
\end{tabular}

Table 4.1: Flow velocities. Typical average flow velocities $u$ in $\mathrm{mm} \mathrm{s}^{-1}$ applied during the different procedures of device operation. In the zig-zag pattern, $u_{\text {aq } 1}$ is increased linearly from 0 to $9.4 \mathrm{~mm} \mathrm{~s}^{-1}$ within $7.5 \mathrm{~s}$, and subsequently, it is decreased again to 0 in the same time. The pattern is repeated leading to a periodicity of $15 \mathrm{~s}$. Details on the zig-zag flow velocities are further discussed in Sec. 6.1. 


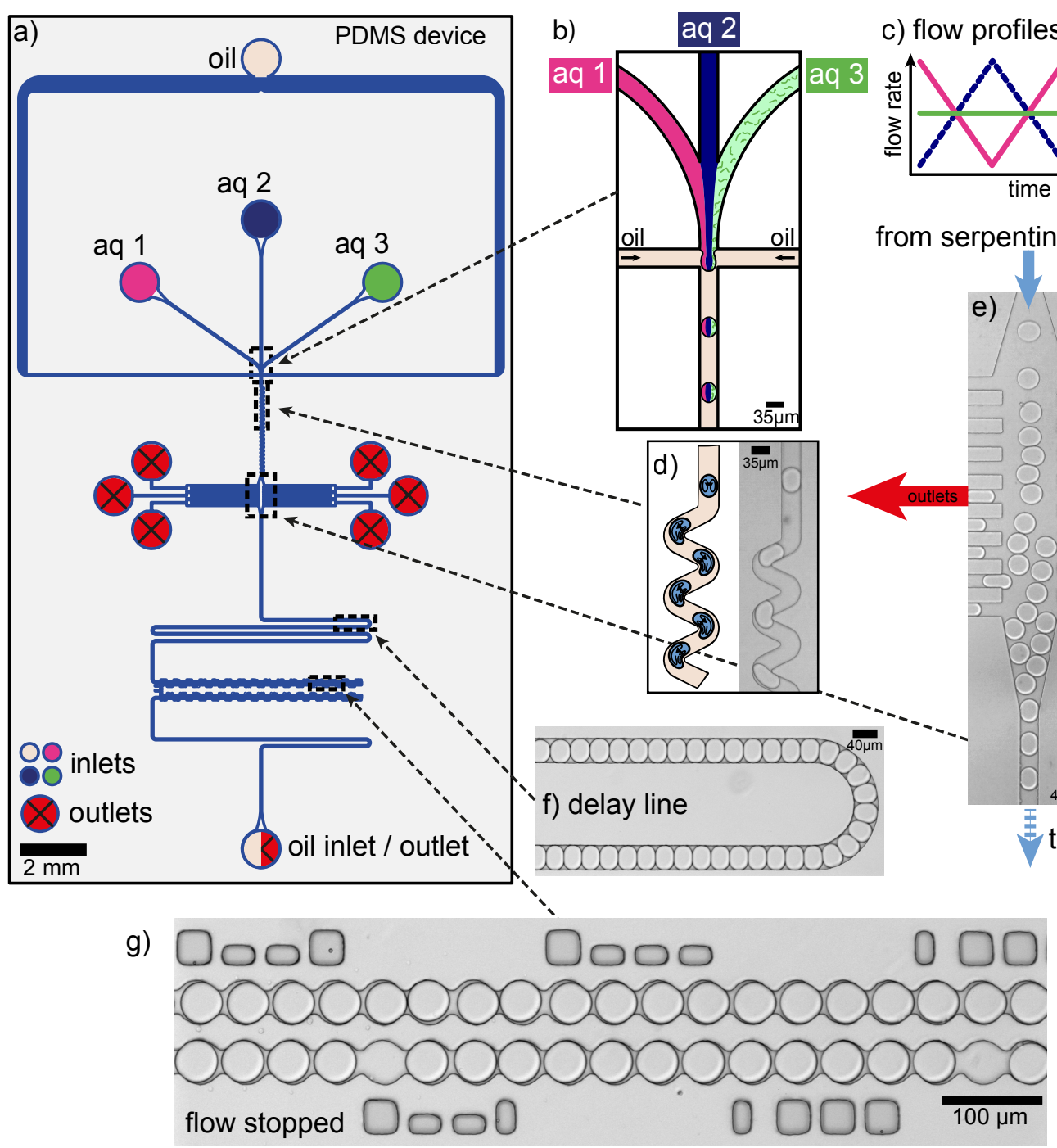

Figure 4.1: Concept of the device $\mathbf{C}$. The aim is to image vimentin protein filaments at different magnesium concentrations. For this purpose, the device consists of several modules for fluid manipulation. In the drop composer (b), three aqueous fluids (aq 1, aq 2, aq 3) join up shortly before they are encapsulated into drops by focusing them with a lateral oil flow. $[7,118]$ c) A zig-zag flow profile is applied for aq 1 and aq 2. d) The drops pass a serpentine channel, to ensure fast mixing of the drop content. [27] e) In a drop basin, the drops are densified and excess drops are rejected laterally. The bottom of the basin leads to a 'collecting channel'. At the beginning of the experiments, there is an oil inflow from this channel into the basin. Consequently, no drops enter. Drop collection is initiated when this oil flow is inverted (Fig. 4.2). Drops are collected for about one minute into a delay line (f), which ensures smooth drop collection. From the delay line, the drops are conducted to the 'drop storage' (g). In this storage, there are 500 constrictions in the channel walls. [109] When the overall flow in the device is stopped, these constrictions immobilize the drops. The content of the drops is then imaged with fluorescence microscopy. (Adapted from reference [36] with permission from AIP Biomicrofluidics) 
containing fluid (aq 3). The flow rates of the other two components follow a periodic zig-zag course (Fig. 4.1c) and their flow rates taken together are constant. This way monodisperse drops are produced. At the same time, the concentration of the multivalent salt is changed from drop to drop in a defined manner. The drops pass an approximately $3 \mathrm{~mm}$ long serpentine channel (Fig. 4.1d). During this passage they undergo strong internal mixing by means of chaotic advection (Sec. 2.3.3). [27]

The drops are produced at a rate of about $400 \mathrm{~Hz}$ and mixed directly afterwards. The microfluidic system has to be considered as an entity of the syringe pump, the tubing and the channels. For the zig-zag flow rate profiles we have to choose a minimum periodicity of about $15 \mathrm{~s}$, so that the microfluidic system has enough time to respond to the set flow rate profiles. In one period of $15 \mathrm{~s}, 6000$ drops are produced. This number is too large to observe the content in all of these drops with the method we use (see below). Therefore, the number of drops is reduced as the drops are conducted into a region with 18 lateral channels and one central channel (Fig. 4.1e). Most of the drops are rejected in this 'basin' as they flow into one of the lateral channels, which lead to outlets. The most important channel is the channel at the very bottom of the basin. At the beginning of an experiment, we apply an oil inflow to this channel (Fig. 4.2a). As a result, no drops can en-

a)

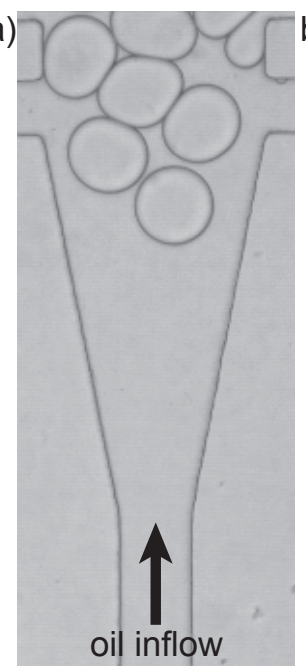

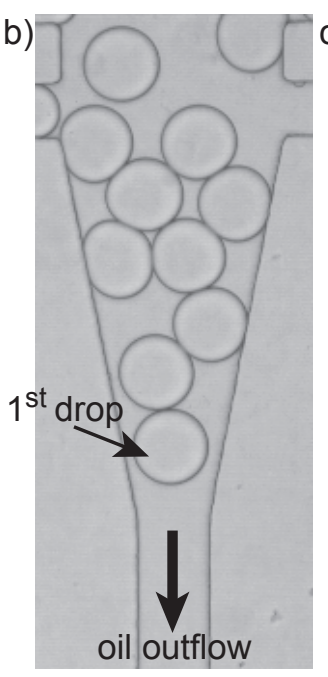

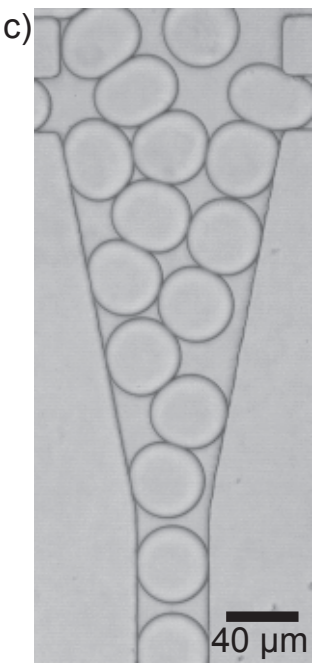

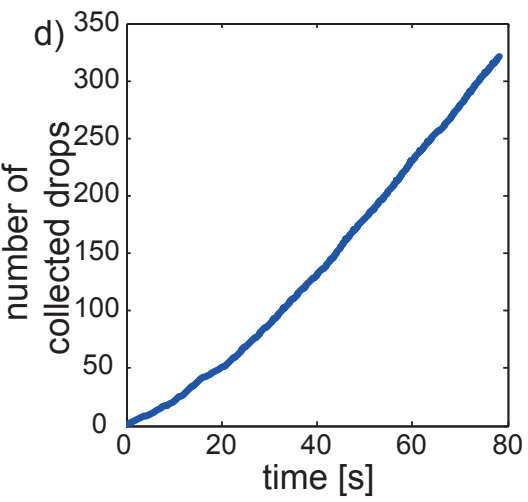

Figure 4.2: Drop collection process. The collection process of drops is initiated when the inflow into the drop basin from the collecting channel (a) is inverted to an outflow (b). After a short delay time the drops begin to enter the collecting channel. This process is recorded in detail with brightfield microscopy. ( $b$ and $c$ ). The details of the collection process are important for calculating the composition of each collected drop later on. d) For this purpose, the number of collected drops as a function of time is analyzed (Sec. 6.1). 
ter. A drop 'collection process' is initiated as the oil inflow is changed to negative values. Then the drops are collected for about one minute (Fig. 4.2b/c) and enter a straight channel, which is used as a delay line (Fig. 4.1f). Its straight channel walls (compared to the drop storage region, see below) allows for a smooth drop collection. The drops enter collection channel at a rate of $5 \mathrm{~Hz}$. Therefore about $1 \%$ of the initially produced drops is collected. Since there are at maximum three drops in parallel in the basin, the chronological drop order is preserved to a great extent in the collection process. [34]

The drop collection process is recorded in brightfield microscopy (Fig. 4.2b-d). This image series is used to determine the exact drop composition for each investigated drop (Sec. 6.1). When enough drops have been collected into the delay line, the oil syringe at the bottom is controlled by hand and the drops are conducted into a channel with constrictions at the channel walls (Fig. 4.1g and 4.3) giving the ability to trap 500 drops at maximum (see Sec. 2.3.2). [109]. All tubings are then cut-off, which stops the flow all over the device. The drop content can be imaged for a few hours, due to the water saturation of the PDMS device (Sec. 3.2.2 and 5.2).

With this procedure not all of the positions are occupied by the drops. For the experiments with vimentin we have about half the positions in the drop storage occupied (Fig. 4.3b). When the drops are stored in the constrictions, up to eight drops are imaged in parallel. All drops are recorded, as we take the images consecutively (Fig. 4.3b). This procedure takes about 1-2 $\mathrm{h}$ time when the whole storage region is imaged. Orientation marks next to the channel walls (Fig. 4.1f) have proven necessary during image acquisition and data analysis. [34]

\subsubsection{Application to vimentin aggregation}

The device $C$ is used to encapsulate vimentin in drops and image the behavior of the protein at different magnesium chloride concentrations. Prior to the experiment, vimentin is assembled to filaments following assembly protocol A (Sec. 3.1.3) and used as component 'aq 3'. This component 'aq 3' is introduced during the device startup by using the 'staggered fluid method' (Sec. 5.3). Component 'aq 1' contains $31 \mathrm{mM} \mathrm{MgCl}$ and 'aq 2' no multivalent ions (Sec. 3.3). The contents of the drops are given in Fig. 4.3. A $40 \times$ objective is sufficient to image a $217 \times 165 \mu \mathrm{m}^{2}$ sized field-of-view with brightfield or epifluorescence. Using this 
method, we image up to 8 drops in parallel from two neighboring parts of the drop storage channel (Fig. 4.3c). We take both a composite image of brightfield and fluorescence microscopy as well as a fluorescence image only. The former is used for orientation in the storage and the latter is used for analysis of the vimentin network. The individual images are stitched together to get an overview

a) The pathway of the drop storage channel

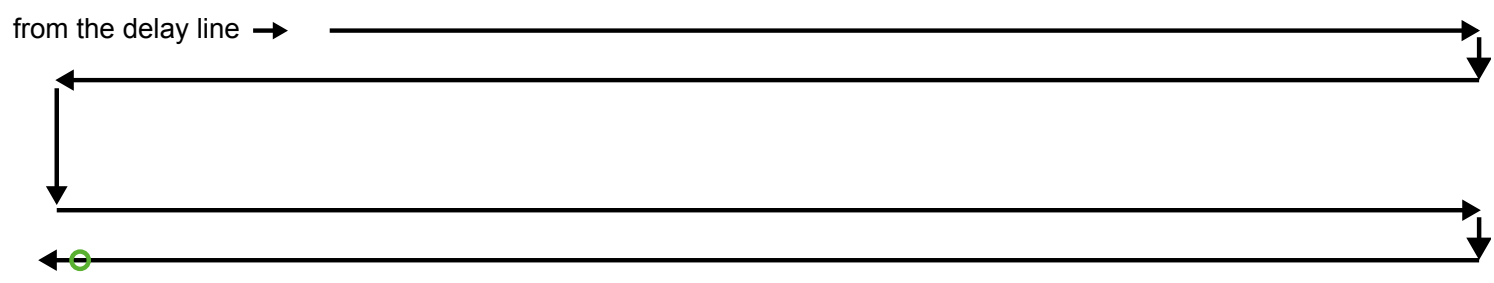

b) An overview of the drops

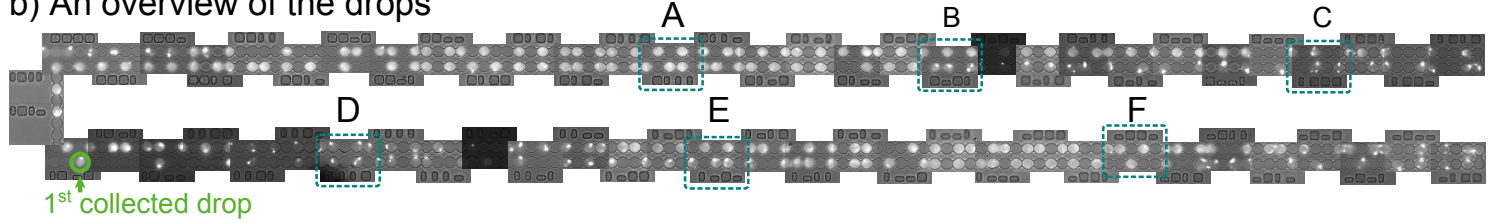

c) Selected regions in detail
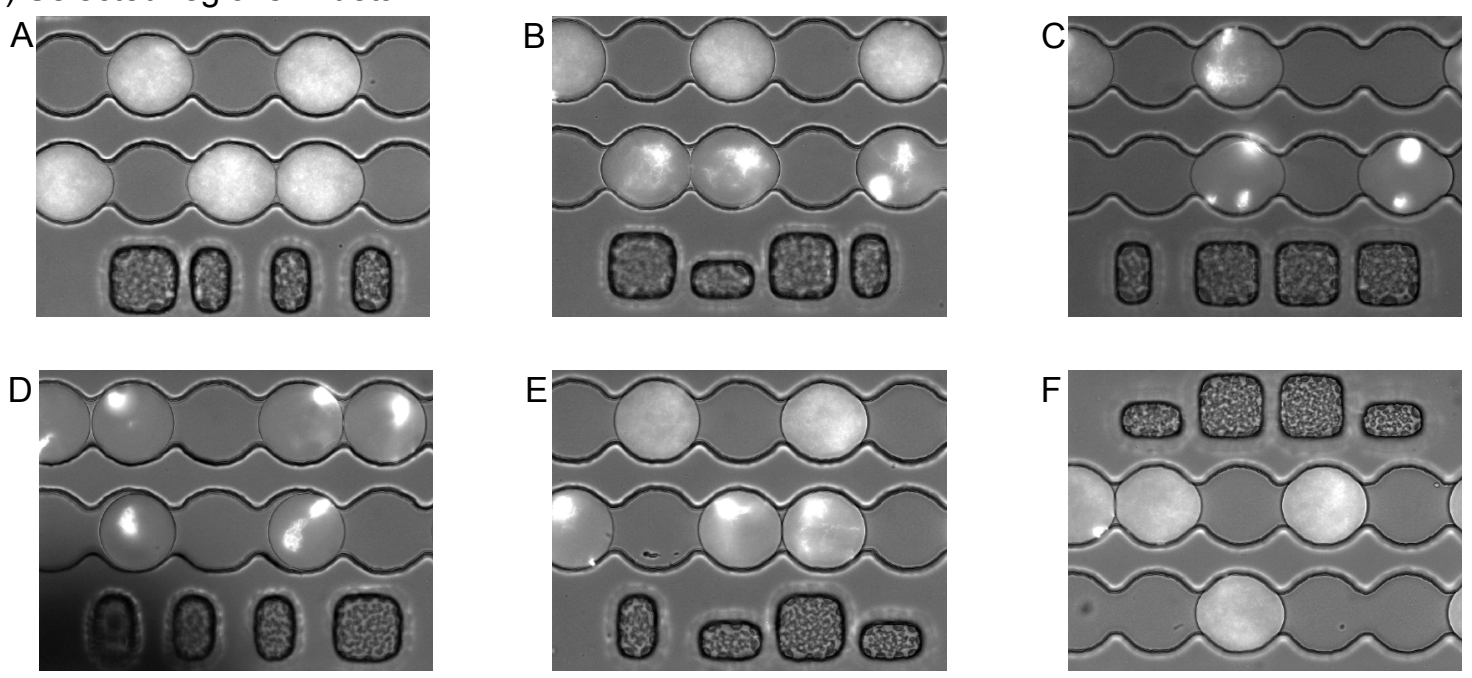

Figure 4.3: Overview of encapsulated vimentin in a device C. According to the pathway of the drop storage channel given in a), the collected drops pass the storage in a chronological order. The first collected drop is the leading drop (green circle, see also Fig. 4.2). In b) an overview of the drop storage from one experiment is given. This overview consists of images that have a field of view to capture a maximum of eight drops at once. They are brightfield and fluorescence composites to visualize channel geometry and vimentin aggregation in parallel. They are stitched together for the overview. c) In the experiment we have different magnesium concentrations in the drops. Therefore, the images A-F show qualitatively different network morphologies. A detailed analysis of this type of experiment is presented in Sec. 7.1. 
of the vimentin aggregation for the whole storage (Fig. 4.3b). This overview shows a qualitative difference between the vimentin networks within the drop series in the storage. It is shown in a detailed analysis in Sec. 7.1 that this difference is due to the different magnesium concentrations in the drops of the whole drop series. Note that in two neighboring channels, the drops do not necessarily have the same magnesium concentration due to the pathway of the drop storage channel (Fig. 4.3a). Therefore, there is a qualitative difference in the vimentin aggregates (Fig. 4.3c-B: compare networks in the upper (magnesium concentration is $\approx 6 \mathrm{mM})$ and lower $(\approx 12 \mathrm{mM})$ channels in the drops).

\subsection{Device T: Time-lapse studies}

In this section, we describe the properties of the device $T$ in which the dependence of protein aggregation is investigated as a function of time at fixed multivalent ion concentrations. We published the microfluidic concept as well as the results on vimentin (Sec. 6.2 and parts of Sec. 7 and 8) in Lab on a Chip. [35]

\subsubsection{Concept}

The concept of the device $\mathrm{T}$ shares the first steps with the device C (Sec. 4.1): Protein and multivalent ions are encapsulated as microfluidic droplets. Shortly before the drops are formed, protein and multivalent ions get in contact for the first time. The content is mixed quickly. Then an individual drop is trapped and its content is imaged for several minutes.

A key feature of this device is that it provides imaging of the interaction of protein and the multivalent ions rapidly after the first protein-salt contact at time $t_{\text {init }}$. 'Rapidly' means that data are recorded already after 1-5 s. Rapid content imaging could also be achieved faster by following drops along a serpentine channel. [118] However, the details of filaments in the moving drops are smeared out as the drops move through the channel. Our approach provides both rapid imaging and good imaging results of the drop content over time. This combination of a rapid imaging start and a trapped drop is the main benefit of this microfluidic concept. 


\subsubsection{Device design and function}

The microfluidic device design to image the drop content rapidly after drop production is shown in Fig. 4.4. Aqueous drops are produced in a flow focusing geometry. [7] Similar to the device $C$, the aqueous phase is composed of three aqueous components aq 1 , aq 2 and aq 3 that join up into one channel shortly before the aqueous phase is dispersed (Fig. 4.4b). [118] The aq 3 component is supplied using the method of staggered fluids (Sec 5.3). After their production, the drops pass a $2.5 \mathrm{~mm}$ long and straight channel (Fig. 4.4a and c). In this channel, the drops are plug-like and - like the serpentine channel in the device $\mathrm{C}$ -

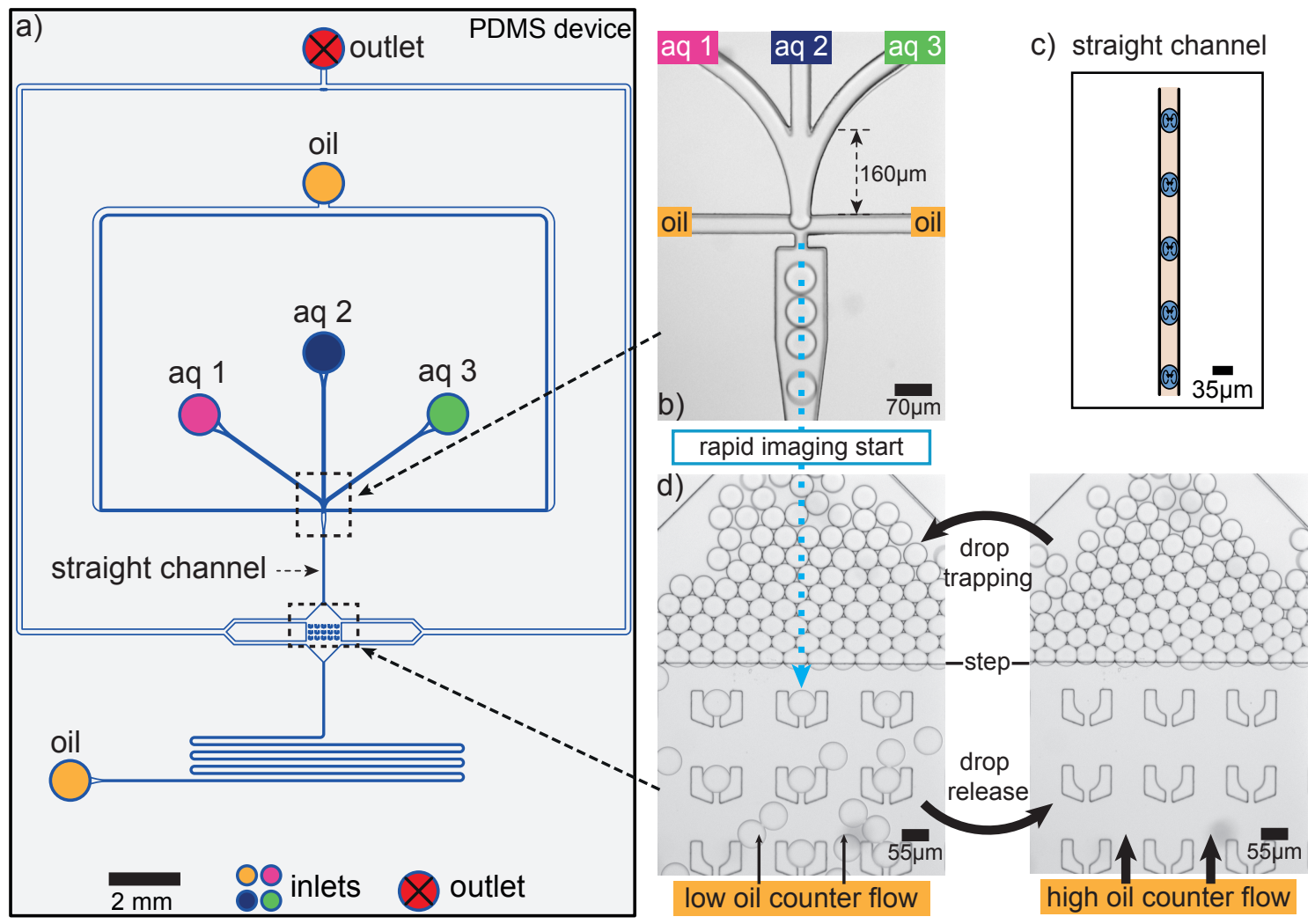

Figure 4.4: Concept of the device $\mathbf{T}$. The aim of the device $\mathrm{T}$ is to image individual drops containing vimentin and a multivalent salt over time and rapidly after the first contact of the multivalent salt and the protein. b) The three aqueous components get into contact shortly before they are encapsulated as drops. [118] c) Efficient mixing is achieved as the plug-like drops move through a straight channel. [27] d) In 'U'-shaped traps [58], the content of drops is imaged over time. The trap region is flat because of a step in the channel. The reduced channel height improves the stability of drop trapping. To empty the traps, a higher counter oil flow can be applied. This allows for serial drop imaging. (Adapted from reference [35] with permission from The Royal Society of Chemistry) 


\begin{tabular}{cccccc}
\hline procedure & $u_{\text {top oil }}$ & $u_{\text {bottom oil }}$ & $u_{\text {aq } 1}$ & $u_{\text {aq } 2}$ & $u_{\text {aq } 3}$ \\
\hline device startup for $\approx 1 \mathrm{~h}$ & 2.4 & 4.8 & 1.2 & 1.2 & 2.4 \\
drop trapping and imaging & 2.4 & 24 & 1.2 & 1.2 & 1.2 \\
drop release for $\approx 10 \mathrm{~s}$ & 2.4 & $\approx 48$ & 1.2 & 1.2 & 1.2 \\
\hline
\end{tabular}

Table 4.2: Flow velocities. Typical flow velocities $u$ in $\mathrm{mm} \mathrm{s}^{-1}$ applied during the different procedures of the device $\mathrm{T}$ operation. They apply for imaging of drops which have a diameter of $100 \mu \mathrm{m}$.

it used to achieve fast mixing. [27] In contrast to the device $C$, the drops within the device $T$ have a larger volume at the flow speeds we typically use (Tab. 4.2). Therefore, their plug-length would span over several serpentine windings, if they passed a serpentine channel as in the device $C$ (Fig. 4.1d). The fast moving drops would most likely break up into smaller drops. Therefore, we use a mixing channel in the device $\mathrm{T}$ that is straight (Fig. 4.4c). Like in the case of the serpentine channel, fast mixing of the drop content is achieved. By calculation the mixing should be finished within two seconds after drop production (Sec. 2.3.3; calculation in Sec. 5.1).

After the straight channel, the drops enter a broadened region where they are densified as most of the oil and excess drops leave the device via two lateral outlet channels (Fig. 4.4d). In the center of the broadened region the drops face a step in the channel. At this step the channel height is reduced from $33 \mu \mathrm{m}$ to $18 \mu \mathrm{m}$. The drops accumulate in front of this barrier and they are continuously exchanged by newly arriving drops. The adjoining flat region has several ' $U$ 'shaped traps. [58] When the oil counter flow from the bottom is relatively low (Fig. 4.4a and d), drops enter the flat region and flow into the traps. In this position, the drop content is imaged over time. The dwell time of the drops in the traps can be more than $10 \mathrm{~min}$. However, this time shows large variation (Sec. 4.2.3) due to the small slit at the lower end of the traps. During drop content imaging, the flow in the device is not stopped. As a result the drops are sometimes squeezed through this slit. At the same time, the slits are needed to empty the traps by an increase in the bottom oil flow (Fig. 4.4d). This allows for a serial imaging of the drop content. A further improvement of the device T could be an adjustment of the size of the slit. A smaller slit would increase the time in the drop. At the same time, the slit has to be large enough to ensure trapping and release of the drops.

The first row of the trap region is the most important one, as it is the fastest row 

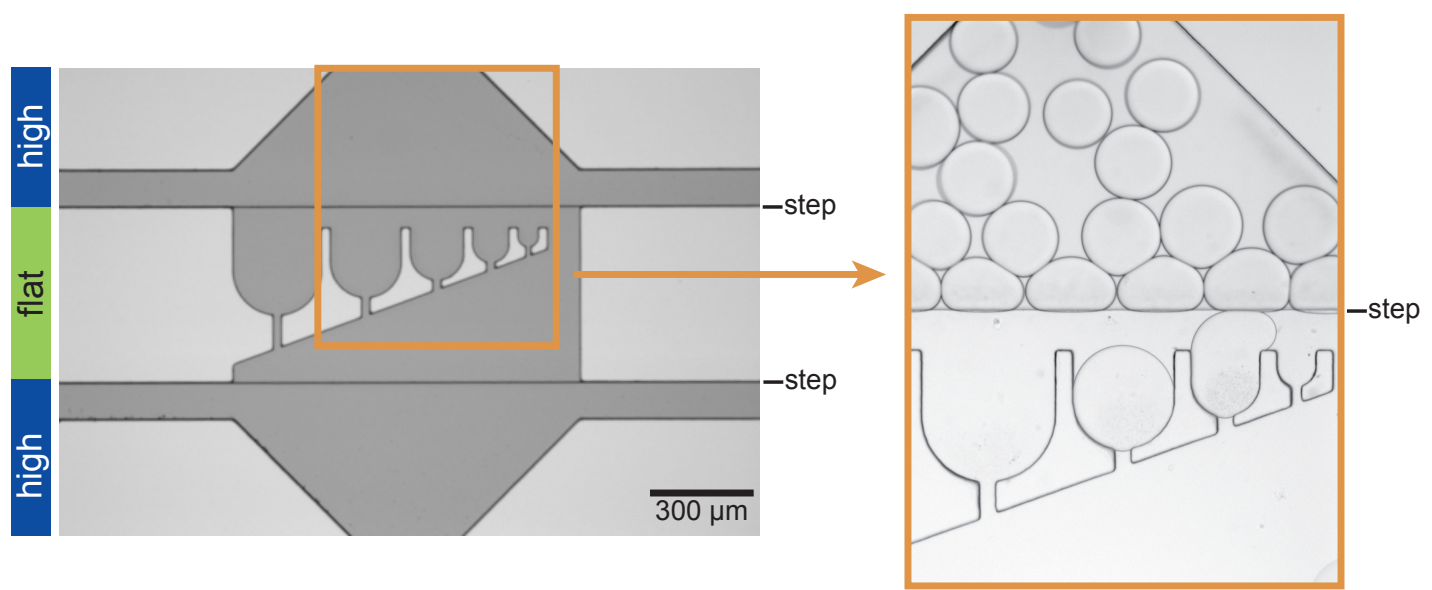

Figure 4.5: Multiple-sized drop traps. A device $\mathrm{T}$ that can trap drops with a diameter of $55 \mu \mathrm{m}$ up to $250 \mu \mathrm{m}$. The drops are imaged in the traps that fit best to the drop diameter. Drops that do not fit the trap size are ignored during imaging. (left: lithography master, right: corresponding PDMS device)

reached. The time that passes between the first contact of the aqueous components and the first image acquisition of a drop content is about 1-5 s (Sec. 4.2.3). The flat region in the device is essential. First, it renders the drop trapping more stable. Second, it reduces the number of incoming drops that could disturb drop content imaging. We find that the trapping mechanism is not restricted to a certain drop size. Complementary to the usage of one trap size as given in Fig. 4.4, a design with traps of different sizes works, too. This way it can be decided in each experiment, which drop size is of interest (Fig. 4.5). For drops of about $100 \mu \mathrm{m}$ in diameter (flat region), we give typical flow speeds in the device in Tab. 4.2.

\subsubsection{Trapping characteristics}

\section{The time from drop formation to the first image}

For the concept of the device $\mathrm{T}$ it is important to know how much time $t_{0 \rightarrow \text { trap }}$ passes between the drop production and the point in time when the drop content is imaged. The first interaction of the drop content can be seen as the point in time of drop production. This aspect is related to mixing and is further discussed in Sec. 5.1. Given the flow speeds in Tab. $4.2, t_{0 \rightarrow \text { trap }}$ is estimated. For the travel through the straight channel, the drops need $0.3 \mathrm{~s}$. They pass the broadened region in front of the trap region within $0.8 \mathrm{~s}$ (measurement of mean drop speed in this region). In the flat trap region, the drop speed is on the order of $10^{-4}$ to 
$10^{-3} \mathrm{~m} / \mathrm{s}$. This gives an additional time of about $1 \mathrm{~s}$ until the drops reach a trap in the first row. In sum, this rough estimate gives a travel time of the drops of about 2 s. However, further deviations from this value should be considered. On the one hand, this time could be decreased by an increase of the overall flow velocity in the device. On the other hand, it takes some extra time until slow drops reach the traps on the plateau. Consequently, it is most reasonable to give an approximation: $t_{0 \rightarrow \text { trap }}=1-5 \mathrm{~s}$.

\section{The dwell time in the traps}

The analysis of the dwell time of the drops shows two classes of flow regimes that are correlated to the dwell time of the drops in the traps. The first flow regime leads to short dwell times of the drops in the traps. In this regime, the drops just stay a few seconds in the traps until they leave it via the small slit. On the one hand, this is a disadvantage, when one aims at imaging the content of a drop as long as possible. On the other hand, this is an advantage, when the content of a drop already indicates the quality of aggregation in the drop within the short dwell time. Then many drops are imaged in sequence, which leads to good statistics. This concept is exploited in Sec. 7.2.1 (Fig. 7.3 and 7.4).

In the second flow regime, very stable drop trapping is possible and the release of
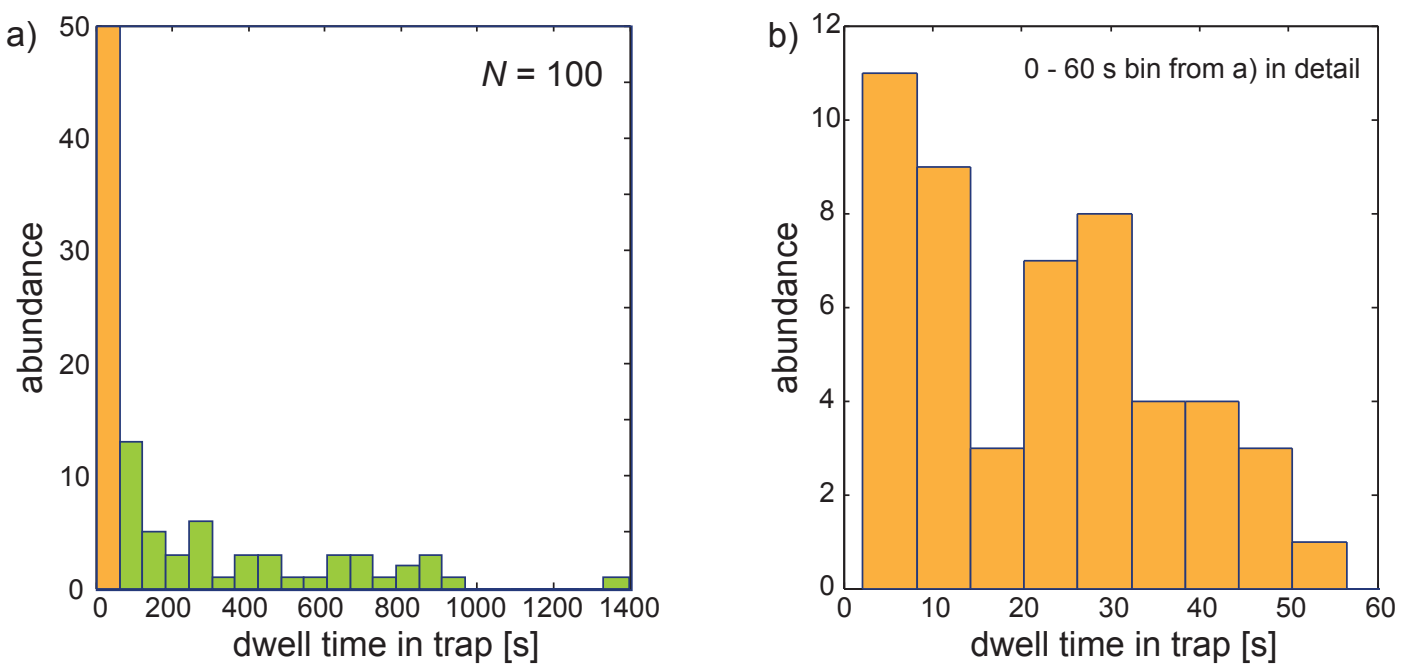

Figure 4.6: Dwell time of drops in the traps. a) For $N=100$ drops the dwell time in the ' $U$ '-traps is analyzed (bin width is $60 \mathrm{~s}$ ). b) The 50 drops from the first bin in a) (0-60 s) are shown in detail. 


\section{MICROFLUIDIC DEVICES FOR PROTEIN STUDIES}

trapped drops is initiated by application of the drop release mechanics by a high counter oil flow. Within this regime, long time-lapse studies can be achieved. Accordingly, the distribution of dwell times accounts for this qualitative picture (Fig. 4.6). About half of the drops show a dwell time of less than $60 \mathrm{~s}$. 


\section{5 \\ Vimentin in drop devices}

\subsection{Mixing characteristics}

Mixing effects in the device $\mathrm{C}$ as well as the device $\mathrm{T}$ are important, as protein structures will be influenced by local inhomogeneities in the distributions of multivalent ions or the protein itself. The diffusion of the multivalent cations is important as well as the diffusion of the filaments. The diffusion constant of the small cations is given by their hydrated radius leading to the diffusion constants $D$ given numerically in Tab. 5.1 ${ }^{1}$. The semiflexible vimentin filaments are

\begin{tabular}{cc}
\hline ion & $D\left[\mathrm{~m}^{2} / \mathrm{s}\right]$ \\
\hline $\mathrm{Mg}^{2+}$ & $7 \times 10^{-10}[138]$ \\
$\mathrm{Co}\left(\mathrm{NH}_{3}\right)_{6}^{3+}$ & $9 \times 10^{-10}[138]$ \\
\hline
\end{tabular}

Table 5.1: Diffusion constants for metal ions in aqueous solutions at $25^{\circ} \mathrm{C}$.

not globular and their diffusion constant is approximated by the consideration of the diffusion of a cylinder. An approximation of the diffusion constant will be sufficient, as the filament length follows a broad distribution (Fig. 7.10). For a cylinder diffusion parallel to its rod axis $(\|)$, perpendicular $(\perp)$ and rotational diffusion (rot) has to be considered. When $L$ is the cylinder length and $d$ its diameter

\footnotetext{
${ }^{1}$ Tang et al. point out that data on the hydrated radii of metals ions (and therefore also the diffusion constant) vary in literature. [132] Consequently, the given diffusion constants should be understood as approximations. In any case, the order of magnitude for the diffusion constants is realistic. For the discussions found here the accuracy will be sufficient.
} 
the governing equations are [74]:

$$
\begin{aligned}
D_{\|} & =k_{\mathrm{B}} T \frac{\ln (L / d)+\gamma_{\|}}{2 \pi \eta L}, \\
D_{\perp} & =k_{\mathrm{B}} T \frac{\ln (L / d)+\gamma_{\perp}}{4 \pi \eta L}, \\
D_{\text {rot }} & =3 k_{\mathrm{B}} T \frac{\ln (L / d)+\gamma_{\mathrm{rot}}}{\pi \eta L^{3}}, \\
D_{\text {trans }} & =k_{\mathrm{B}} T \frac{3 \ln (L / d)+2 \gamma_{\|}+\gamma_{\perp}}{4 \pi \eta L} .
\end{aligned}
$$

Herein, the addition of the parallel and perpendicular diffusion give a combined translational diffusion constant $D_{\text {trans. }}$. For the case $L / d=\infty$, the coefficients $\gamma$ are given as $\gamma_{\|}=-0.114, \gamma_{\perp}=0.886$ and $\gamma_{\text {rot }}=-0.447$. [74] Practically, the filaments are much longer than their diameter, e.g. $L / d=100$ for a filament of $1 \mu \mathrm{m}$ length. Therefore, the application of the limiting case $L / d=\infty$ will lead to a good approximation of the diffusion constants (Fig. 5.1a). For filament lengths where the condition does not hold $(L / d \approx 1)$, the diffusion constant $D$ is underestimated and therefore diffusion times overestimated. As the drops in our microfluidic devices are oblate (18 $\mu \mathrm{m}$ drop height compared to about $40 \mu \mathrm{m}$ and $100 \mu \mathrm{m}$ diameter, respectively), we consider a two dimensional diffusion of the filaments. The times $t$ for traveling a distance $x$ are given for several filament
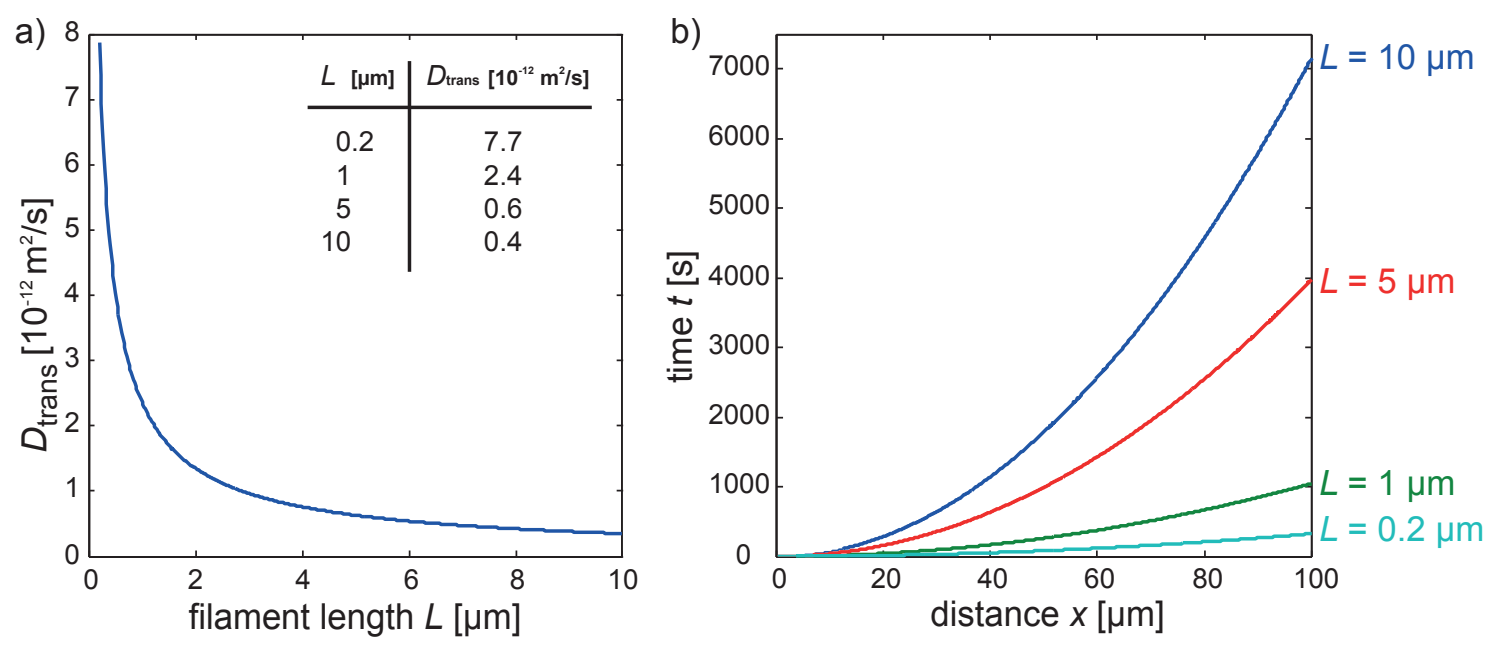

Figure 5.1: Diffusion constants of vimentin filaments. a) Estimate of the translational diffusion constant $D_{\text {trans }}$ of vimentin as a function of filament length $L$. b) Time $t$ required to pass a distance $x=\sqrt{4 D_{\text {trans }} t}$ by two dimensional diffusion for different filament lengths. 

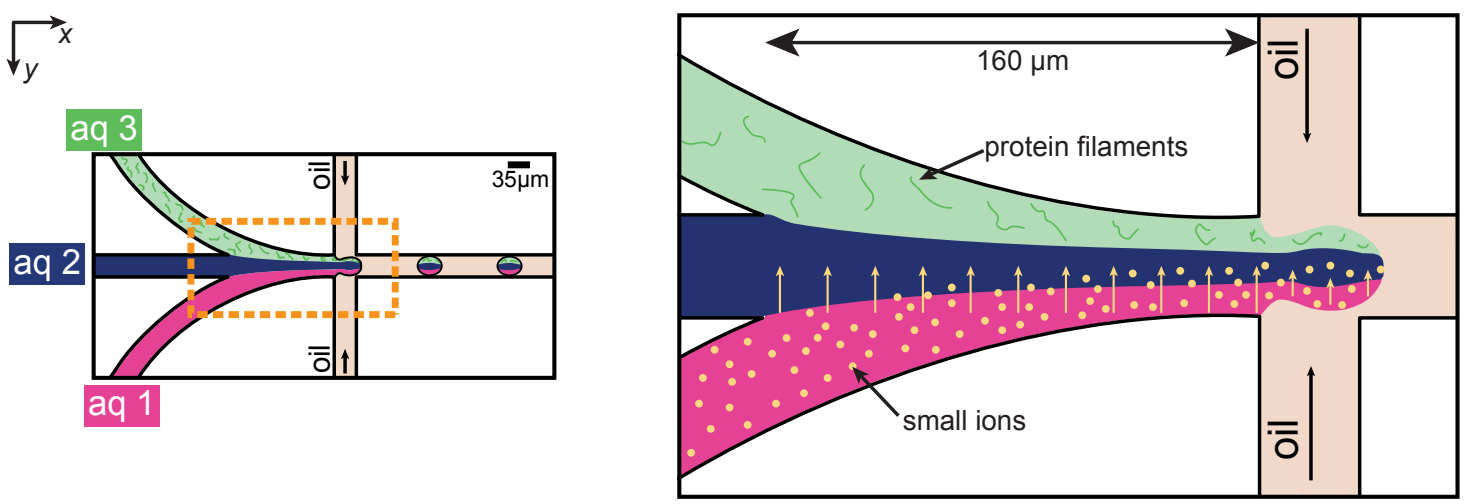

Figure 5.2: Side-by-side flow. Before the aqueous phases are encapsulated as drops, they stream in a side-by-side manner. [134] During this phase, only the small ions diffuse significantly, as the large filaments diffuse slowly.

lengths in Fig. 5.1b.

We now investigate the effect of mixing in the drop system. For this purpose, we consider the two steps, in which mixing effects take place in the drops: mixing between the parallel flowing liquids before they are in the drop (Fig. 5.2) and the time when the plug-like drops move along the channel. In the first part of mixing, the three aqueous fluids flow in a side-by-side manner over a distance of $160 \mu \mathrm{m}$. For the device $\mathrm{C}$, this means a passage time of about $t_{\mathrm{C}}=10 \mathrm{~ms}$ and for the device $\mathrm{T}, t_{\mathrm{T}}=50 \mathrm{~ms}$ at the typical flow speeds as given in Tab. 4.1 or Tab. 4.2 , respectively. The diffusion constant for vimentin is comparatively small and the distance traveled by diffusion in $y$-direction is $<1 \mu \mathrm{m}$ for very small filament fragments of $0.2 \mu \mathrm{m}$ length (applies for device $\mathrm{C}$ and device $\mathrm{T}$ ). For small metal ions ('mi') the order of magnitude of the diffusion constant is important. For both magnesium ions and hexammine cobalt it is $D_{\mathrm{mi}} \approx 10^{-9} \mathrm{~m}^{2} / \mathrm{s}$ (Tab. 5.1).

We consider first mixing in the device $C$. Here, the small metal ions diffuse in the side-by-side flow over a distance of $x=\sqrt{2 D_{\mathrm{mi}} t_{\mathrm{C}}}=4 \mu \mathrm{m}$ (one-dimensional diffusion in $y$-direction). As the channel width for the three aqueous components is $35 \mu \mathrm{m}$ in width, the small ions might in some cases reach the protein phase before the drops are formed. This is especially the case when the flow rate of the aq 1 component is higher than the aq 2, due to the smaller striation length (Sec. 2.3.3) of the aq 2 component in the side-by-side flow. However, strong mixing between the fluids will start after drop formation. The drops pass the $4 \mathrm{~mm}$ long serpentine channel (serpentine channel contour length) with a velocity of typically $60 \mathrm{~mm} / \mathrm{s}$ (Fig. 4.1). This mixing passage takes $70 \mathrm{~ms}$. For a $10 \mu \mathrm{m}$-long filament and a channel of $35 \mu \mathrm{m}$ width, the Péclet number is $P e=5.3 \times 10^{6}$ (Eq. 
2.29). The required mixing time can be compared to the mixing time for pure diffusion in the completely immobilized drop by means of Eq. 2.32:

$$
\frac{t_{\text {diff }}}{t_{\text {mix }}}=\frac{P e}{1 \times \log P e}=3.4 \times 10^{5} .
$$

An interesting view on this high acceleration of mixing is the consideration of an effective diffusion constant $D_{\text {eff }}=D \times t_{\text {diff }} / t_{\text {mix }}$. Considering a $10 \mu \mathrm{m}$ long filament with $D=4 \times 10^{-13} \mathrm{~m}^{2} / \mathrm{s}$, it is effectively mixed in the drop in the same time as a (hypothetical) particle with $D_{\text {eff }}=1.4 \times 10^{-7} \mathrm{~m}^{2} / \mathrm{s}$ in a completely immobilized drop. This is an outstanding performance of the mixer, as $D_{\text {eff }}$ is two orders of magnitude larger than for small metal ions (see Tab. 5.1). The resulting mixing time for a $10 \mu \mathrm{m}$ long filament is on the order of $10 \mathrm{~ms}$. Considering also the mixing of the filaments, the drops are completely mixed when they leave the serpentine channel. We considered the slowest component of the system, i.e. a $10 \mu \mathrm{m}$ long filament. All other components are diffusing faster and are therefore mixed even faster. As the diffusion constant of the metal ions is about three orders of magnitude larger than for the filaments, the mixing time for these ions is also orders of magnitude smaller (Eq. 2.28). Therefore, the metal ions are almost instantly distributed all over the drop, once the drop is in the serpentine channel. For the device $\mathrm{T}$, the three aqueous phases flow side-by-side with a lower velocity compared to the device $C$. In these experiments, filaments have an average contour length of $5 \mu \mathrm{m}$ (Fig. 7.10). In the side-by-side flow, metal ions diffuse by $x=\sqrt{2 D_{\mathrm{mit}} t_{\mathrm{T}}}=10 \mu \mathrm{m}$. Consequently, the small metal ions reach the protein containing aqueous phase like in the device $C$. In the device $T$, the protein filament diffusion is also below $1 \mu \mathrm{m}$. The aqueous phases are then encapsulated in drops with a diameter of $\approx 100 \mu \mathrm{m}$ (18 $\mu \mathrm{m}$ channel height). After these drops have passed the straight channel of $2.5 \mathrm{~mm}$ length in $300 \mathrm{~ms}$, the striation length is $1 \mu \mathrm{m}$ (Eq. 2.31). The $5 \mu \mathrm{m}$ long filaments pass this distance within $1 \mathrm{~s}$. Consequently, the drops should all be well mixed also in the device $\mathrm{T}$, when the drop content is imaged in the trap.

In summary, the calculation presented for this mixing process give approximations to the mixing processes. We find that for both approaches - the device $C$ with its serpentine channel as well as the device $\mathrm{T}$ with the straight channel good mixing of the drop content should be accomplished very fast. In direct comparison to the pure diffusion of filaments in an immobilized drop (Fig. 5.1b), mixing in the moving plug-like drops is orders of magnitudes faster. The use of 
serpentine channels is outstanding for mixing, but the use of the straight channels is still sufficient for mixing.

These theoretical calculations predict that the drops are well-mixed. To verify this statement, representative drops produced in the device $\mathrm{T}$ are investigated (Fig. 5.3). A typical observation is that the two drop hemispheres are divided by a stripe of low fluorescence in the center of the drop. This is likely due to the two symmetric vortices that exist when a plug-like drop moves through a straight channel (Fig. 2.11). Overall, the fluorescence and therefore the protein filaments are distributed everywhere in the drops, since the fluorescence is above the background level everywhere in the drops. However, there are regions of

a)
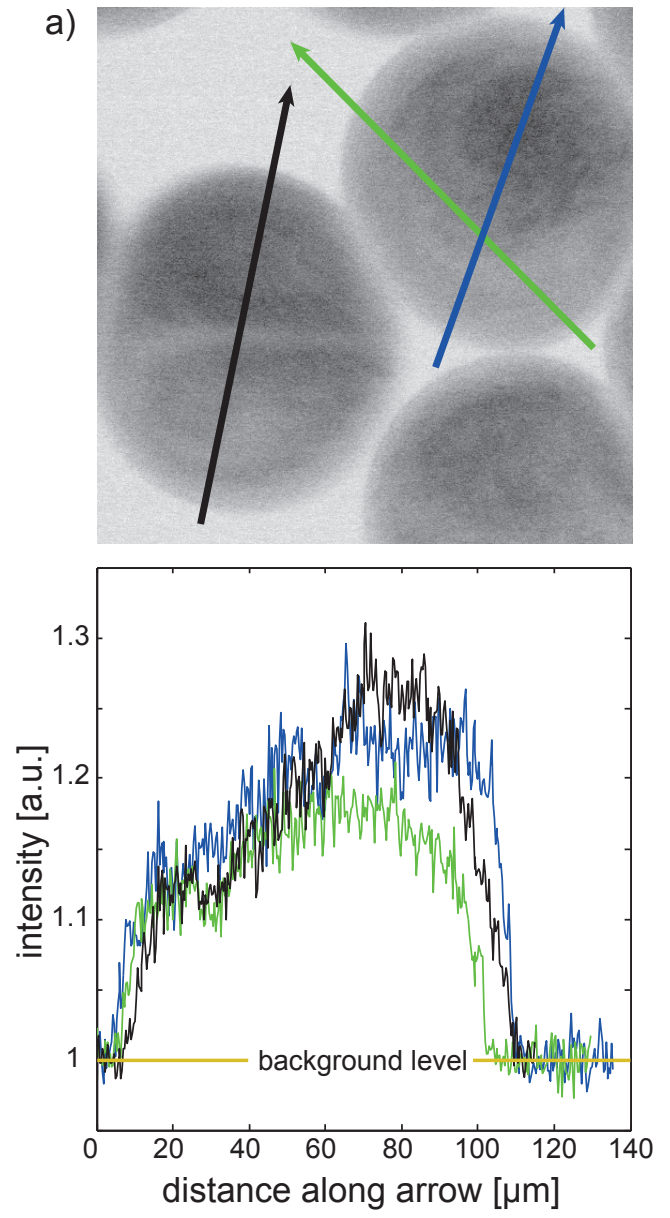

b)
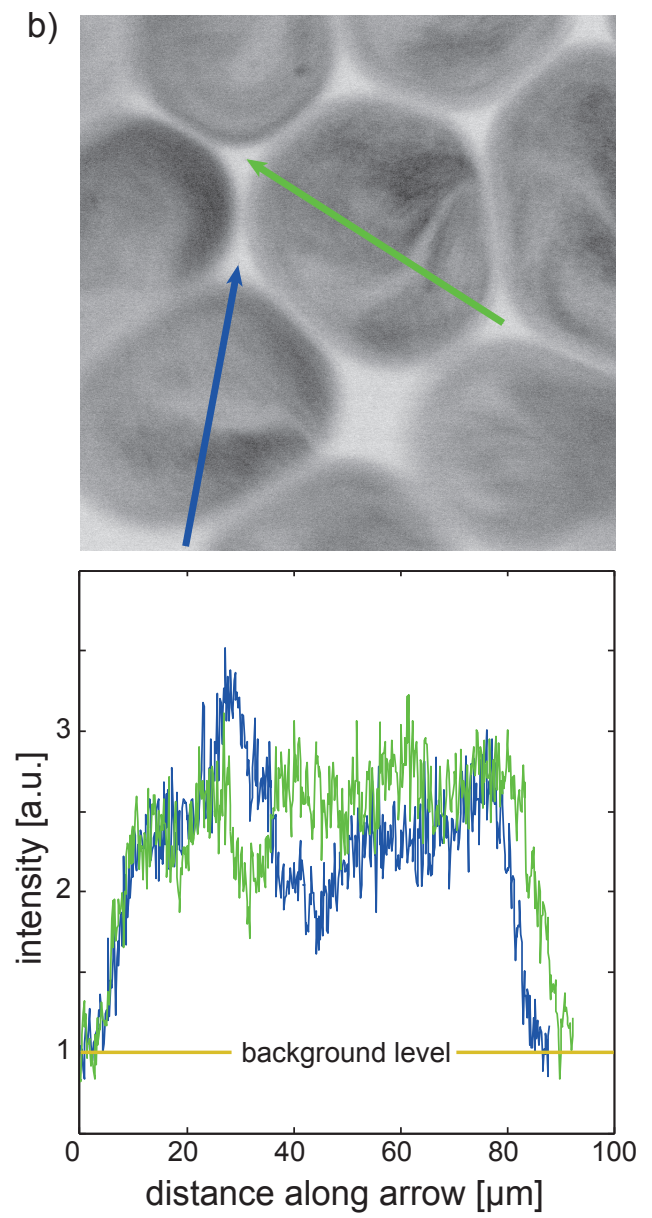

Figure 5.3: Vimentin distribution in drops. Drops containing vimentin filaments imaged during their movement in front of the step in the device $\mathrm{T}$ (compare Fig. 4.4d). In a), no multivalent ions are in the drops, whereas in b), $10 \mathrm{mM}$ magnesium chloride is present. In both cases, the intensity profiles show that there are regions in which the fluorescence is more pronounced. 
pronounced fluorescence. In Fig. 5.3, drops without multivalent ions (a) are compared with drops that contain multivalent ions (b). As there is no observable difference between these two cases, the observed inhomogeneities cannot be accounted for filament aggregation in parallel to the mixing process. A possible explanation for the imperfection of the mixing in the drops are the filamentous properties of vimentin. Steinhauser et al. show that in flow, filaments do not behave like spherical particles. [122] The theory of mixing in drops could therefore be especially valid for small dyes and is likely to be inaccurate for the complex behavior of filaments. Additionally, the effectiveness of mixing can depend on very small details during the drop formation process and gets less effective the longer the plug-like drops are during their movement through the channel. [134] Nevertheless, the drops are well mixed compared to the initial state right after drop production (side-by-side fluids, Fig. 5.2 and 5.6b) and the result of the mixing process is sufficient for our studies of the interaction of vimentin filaments and salts (Sec. 7). This result is also transferable to mixing in the device $\mathrm{C}$, where the mixing performance is better due to the serpentine channel and the smaller drop size. The general problem of mixing is further addressed in the discussion section (9.1.4).

\subsection{Drop volume changes and concentration steadi- ness}

PDMS devices are permeable to water. [20] As a consequence, the aqueous drops can shrink or grow during the time course of the experiment. The devices $C$ are saturated with water during the experiments to prevent drop shrinkage. As a result the drops grow very slightly. In order to quantify this growth, we investigate drops that are kept continuously in humidified atmosphere for a long time after the ordinary experiments were carried out (Fig. 5.4). After $405 \mathrm{~min}$ the drop volume has increased by a factor of 1.28. The relative drop volume $V_{r}(t)$ is given by the volume of the drop after time $t$ compared to the initial volume. Assuming a linear behavior of the drop growth, it is found that $V_{r}(t)=1.2 \times 10^{-5} \mathrm{~s}^{-1} \times t+1$. This assumption of linear growth will somehow overestimate $V_{r}(t)$ for smaller times, as larger drops have more PDMS contact and therefore more water will diffuse into these drops per time. When possible, it is beneficial to avoid water 
a)

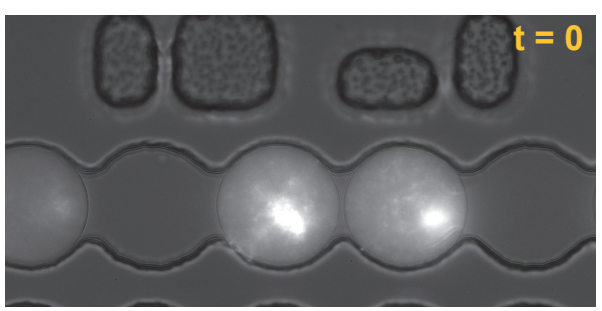

b)

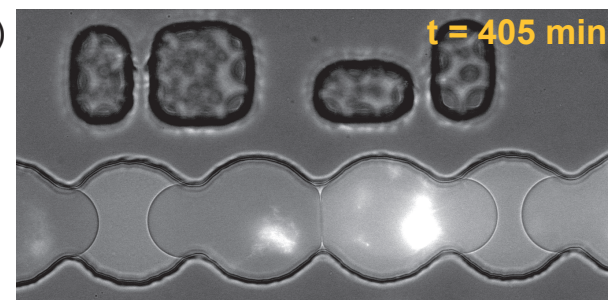

c)

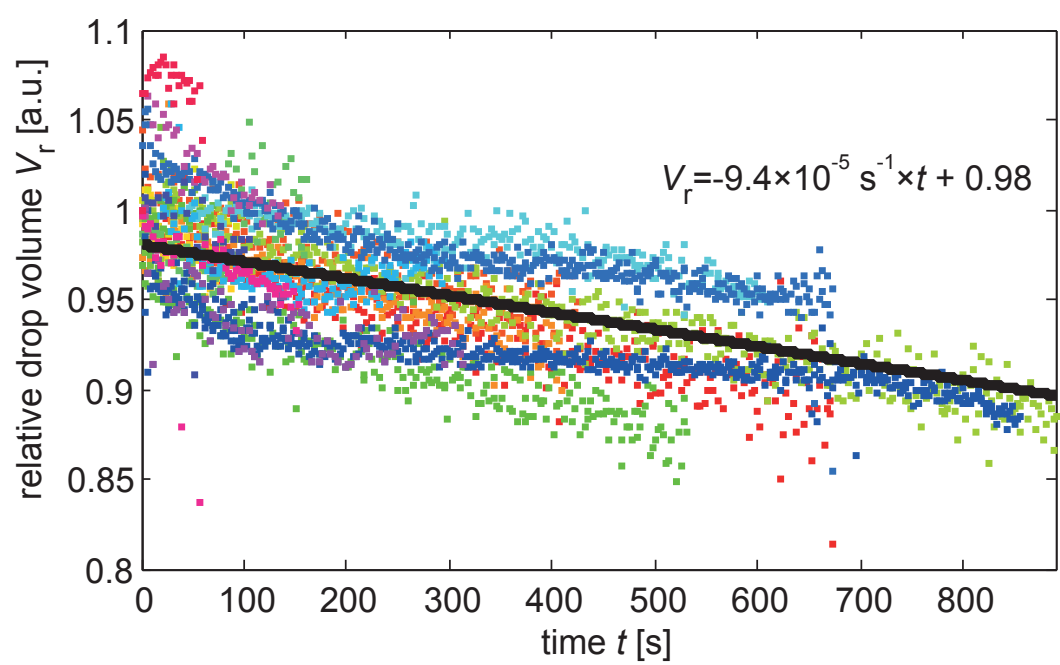

Figure 5.4: Changes of drop volume. a/b) Device C: Long-time study on the drop volume shortly after drop trapping (a) compared to the same drops 405 min later (b). The drop volume increases by a factor of 1.28 . c) Device T: Relative drop volume $V_{\mathrm{r}}$ that is normalized to the drop volume at $t=0$. Each color shows $V_{\mathrm{r}}$ for a different drop. The black curve gives the weighted linear fit for the shrinkage process with a slope of about $-0.1 \frac{\% 0}{s}$.

saturation of the PDMS devices, since this procedure impedes the treatment of the devices with Ombrello (Sec. 3.2.2) and diminishes the overall cleanliness of the devices.

For the device $\mathrm{T}$, the relevant time scales are completely different to those of the device $C$, since the imaging of the drop content is achieved in most cases within $600 \mathrm{~s}$. Therefore, these devices are not water saturated and the shrinkage of the drops is accepted instead. To quantify this shrinkage, we analyze several relative drop volumes on the basis of experiments with aggregating vimentin in the drop in Fig. 5.4. The consequences of drop volume changes are that the concentration 
a)

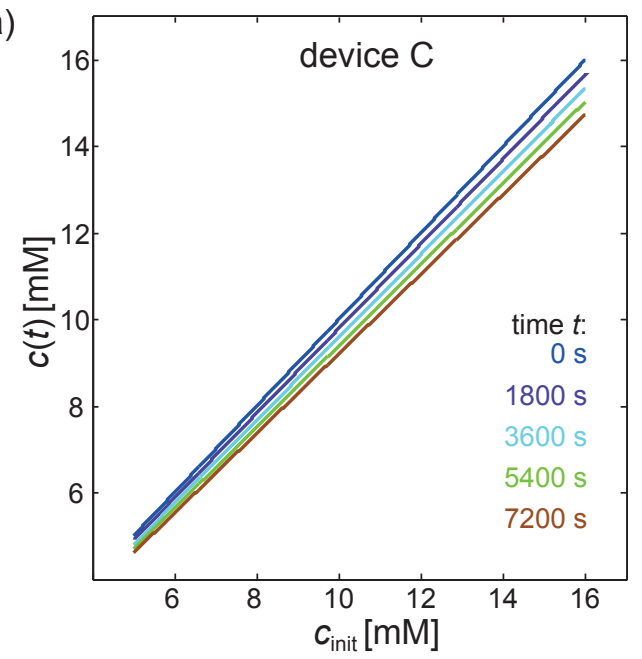

b)

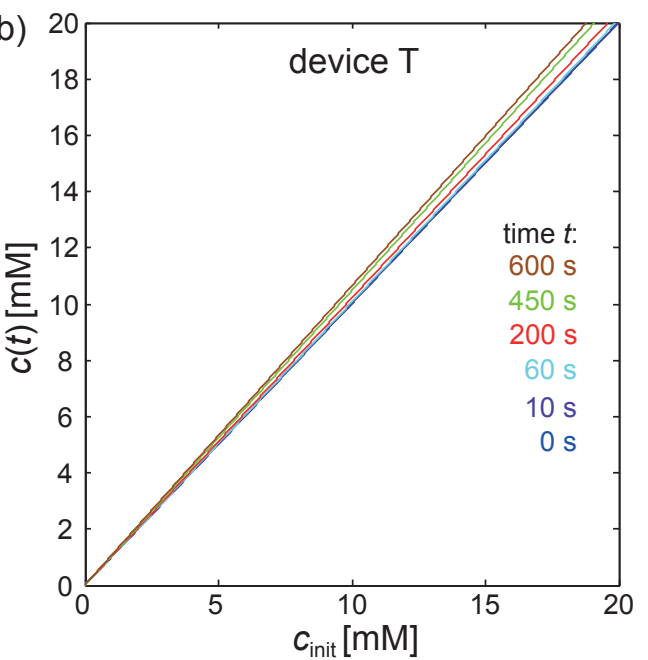

Figure 5.5: Impact of the changes in drop volume. Estimated changes in concentration $c(t)$ due to drop growth (a, device $\mathrm{C}$ ) or shrinkage (b, device $\mathrm{T}$ ). The concentration changes are negligible on the relevant time scales.

of the chemicals inside the drops change according to

$$
c(t)=\frac{c_{\text {init }}}{V_{r}(t)}= \begin{cases}\frac{c_{\text {init }}}{\left(1+1.2 \times 10^{-5} \mathrm{~s}^{-1} \times t\right)} & \text { for the device C } \\ \frac{c_{\text {init }}}{\left(1-0.0001 \mathrm{~s}^{-1} \times t\right)} & \text { for the device } \mathrm{T}^{\prime}\end{cases}
$$

where $c_{\text {init }}$ is the initial concentration in the drop and $V_{r}(t)$ the relative drop volume after a time $t$ compared to the initial drop volume. In Fig. 5.5, we compare concentration changes of magnesium ions for different initial concentrations and different times. A key result is that within the relevant time for the device $C$ or the device $\mathrm{T}(0-2 \mathrm{~h}$ or $600 \mathrm{~s}$, respectively), the changes in magnesium concentration almost never exceed $1 \mathrm{mM}$ and are typically smaller. For instance, an initial concentration of $10 \mathrm{mM}$ turns into 9.79, 9.59, 9.39, 9.20 after 1800, 3600, 5400, $7200 \mathrm{~s}$ for the device $C$ and 10.01, 10.06, 10.20, 10.47, $10.64 \mathrm{mM}$ after 10, 60, 200, 450, $600 \mathrm{~s}$ for the device T. Concentration changes within this range will be negligible for the questions raised here. 


\subsection{The challenge to encapsulate vimentin in drops}

For both the device $\mathrm{C}$ and the device $\mathrm{T}$, the startup procedure of the microfluidic device is the most challenging step during the experiment. In both devices the aqueous component aq 3, which contains the vimentin protein filaments, is the most critical one (Fig. 4.1b and Fig. 4.4b): Vimentin filaments stick firmly to glass and PDMS surfaces as many tests on cover slips have shown. Therefore, component aq 3 tends to cover the channel walls of the microfluidic devices. Further, the risk of channel clogging will notably increase, if vimentin is in contact with multivalent ions before these two components are encapsulated in drops. Especially during the startup of the device, when no stable drop formation is established, this risk is high. Then the multivalent ion salt might accidentally flow into the aq 3 channel and lead to clogging. In Fig. 5.6, a well-established drop production is compared to a channel where protein clogging occurs. To reduce the stickiness of the protein to the channel walls, a channel wall coating might be a solution, preventing that vimentin sticks to the walls. However, we coat the channels walls

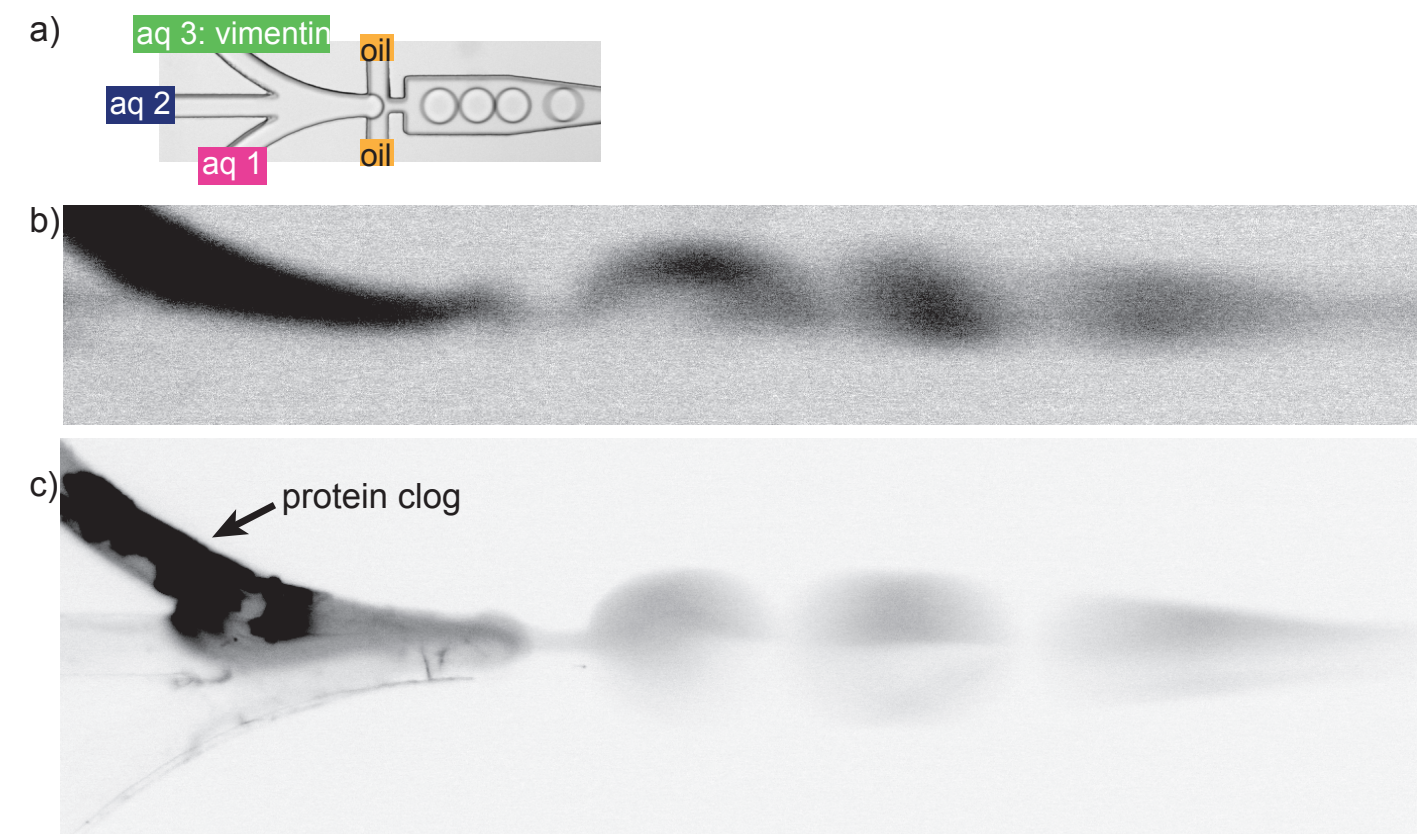

Figure 5.6: Challenges in drop production. a) Well established drop production. b) Inverted grayscale fluorescence image of a well-established drop production, where no protein clog is in the channel. c) A dominant clog in the device that has accumulated over time. In b) and c) the intensities are adjusted differently for illustration purposes: The clog in c) is much brighter compared to any fluorescence in b). 
with Ombrello (Sec. 3.2.2). Therefore, any second channel coating is in competition with that first layer. Secondly, it is not clear which surface treatment could effectively passivate the surfaces against vimentin. Flushing of bovine serum albumin (BSA) through the channels has no effect and instead disturbs the drop production. Sigmacote (Invitrogen), which is successfully used to prevent F-actin from sticking to surfaces, [57] also does not lead to success. However, a change in the microfluidic technique compared to the standard approach is introduced. The straightforward way to supply the aq 3 component is to plug in the respective tubing and then pump the protein solution. When the drop production is not established yet, this can lead to the aforementioned difficulties during the startup procedure of the device. Therefore, a $70 \mathrm{~cm}$ long tubing is used for the aq $3 \mathrm{com}-$ ponent (Fig. 5.7a). The tubing is filled with $45 \mu \mathrm{L}$ protein filament solution first, then with $3 \mu \mathrm{L}$ air cushion and finally with $5 \mu \mathrm{L}$ assembly buffer. When this tubing is plugged into the device and component aq 3 pumped through the device, then first the assembly buffer enters the device, then the air cushion and finally the protein. This way, the drop production is initiated smoothly with the assembly buffer and prevents the clogging of the channel. The air barrier in the tubing prevents premature mixing of assembly buffer and the protein, which could otherwise occur due to Taylor dispersion (Sec. 2.3.3). In Fig. 5.7b, we investigate the operation of this staggered fluid approach directly. Except for a small 'protein leakage' into the assembly buffer, the staggered fluid approach performs well. The air is not encapsulated into drops, but instead flows downstream and the drop production remains unimpaired for the following protein solution. This way the success of an experiment is greatly improved and the procedure was crucial for many results presented here. Although this is an important step for the encapsulation of vimentin protein into drops, it still does not assure the success of an experiment and protein clogging remains a challenge. This aspect is further discussed in Sec. 9.1.5. 
a)

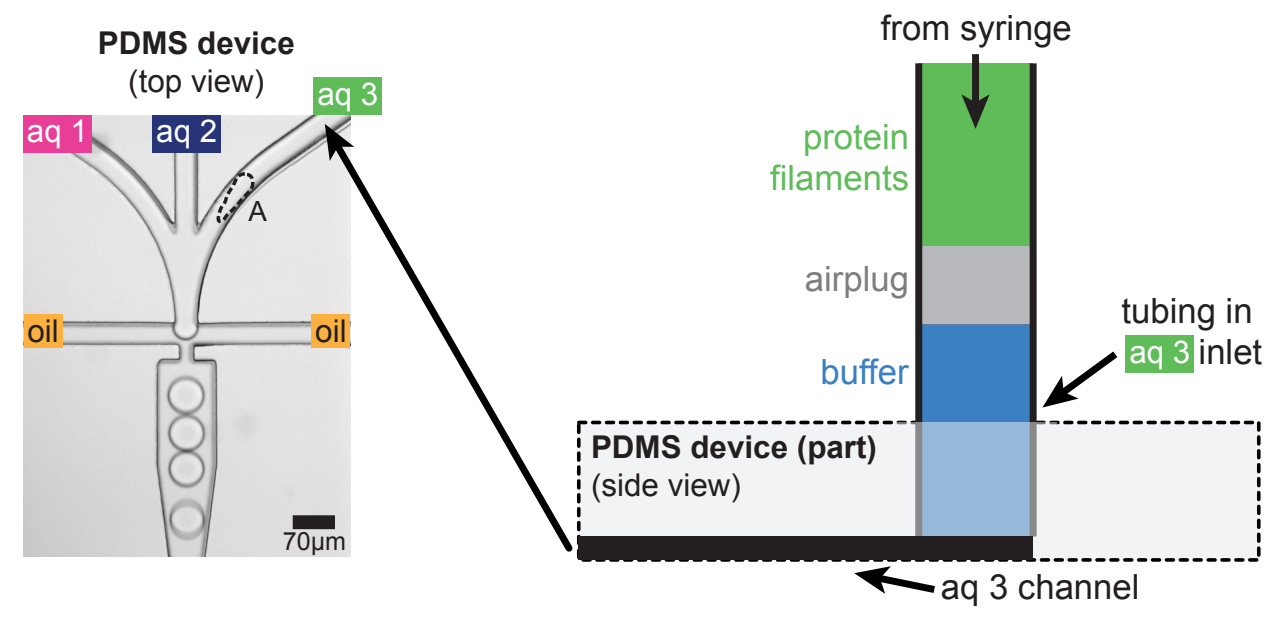

b)

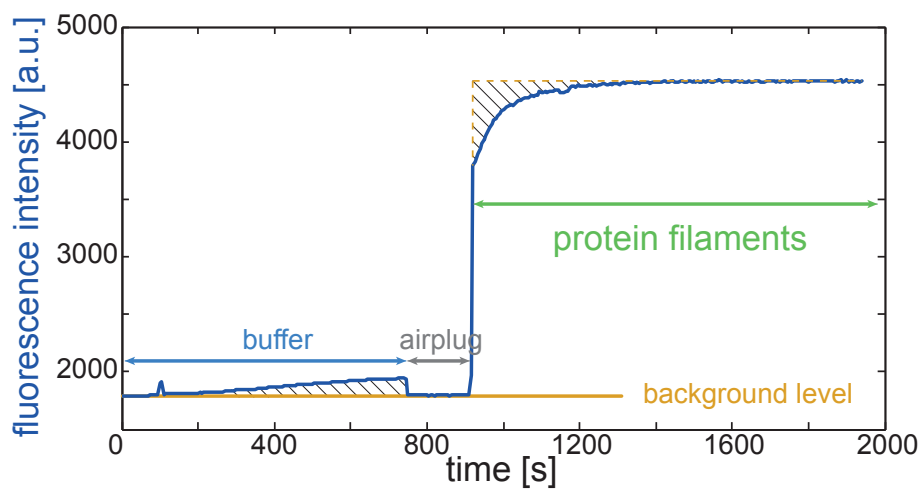

Figure 5.7: Staggered fluid setup. a) During the startup of the device, the supply of the aq 3 component consists of three components that enter the aq 3 channel in this order: assembly buffer, airplug and then protein filaments in assembly buffer. The method is used to ensure a smooth device startup procedure. b) During the startup procedure, the mean fluorescence intensity in the aq 3 channel only is investigated (illustrated area A in (a)). During pumping of the buffer, a small 'fluorescence leakage' is detected leading to fluorescence signal during the time where buffer only should flow. Then, the airplug goes through the channel, and accordingly, no fluorescence is detected. After that, a steep increase in the fluorescence signal indicates the arrival of protein filament solution. Interestingly, the shaded fluorescence area of the 'leakage' is almost of the same size as the area that the signal of the protein is missing at the beginning. 



\section{6 \\ Data analysis}

The analysis of the experimental data from the device $C$ as well as the device $T$ demands sophisticated algorithms. For the device $C$, we present a method to calculate the concentration for each drop in the drop storage (Fig. 4.1g). In order to analyze the experiments with the device $\mathrm{T}$, we need algorithms that quantify network aggregation.

\subsection{Concentration determination in the device C}

The knowledge about pump speeds of the fluids, the delay time of the device, the recording of the drop collection process and the chronological order of the drops in the drop storage is the key to determining the magnesium concentration in each drop that is stored in the drop storage.

Changes in the flow rates for the magnesium chloride and buffer component (aq 1 and aq 2, Fig. $4.1 \mathrm{~b}$ and c) cannot be realized immediately, as there is a lag time in the method, so that flow rates at a certain position in the microfluidic channels are delayed. For instance, the drops do not immediately have the corresponding chemical composition in the moment the flow rates are changed. Let $t_{\mathrm{d}}$ be the time between the setting of a flow rate to the syringe pumps and the time-point when the drops with the corresponding chemical composition reach the collecting channel (Fig. 4.1 and 4.2). This delay time is due to damping effects of the elastic microfluidic device materials as well as the time that drops take to reach the collecting channel.

Further, we use triangular zig-zag shaped pump profiles for the fluids with a pe- 
riodicity of $15 \mathrm{~s}$. As this periodicity is rather short, the pumped flow rates do not reach the extreme values, i.e. the zig-zag (Fig. 4.1c) shaped flow profiles become actually more sine-like ones. This effect can be thought of as an effect of inertia, too. This phenomenon can be understood with the following thought: When the periodicity of the zig-zag pump profile gets smaller and smaller approaching the technical limit of $200 \mathrm{~ms}$ of the syringe pumps, the inertia of the system will not allow the flow rates to be accurately pumped. Instead, the flow rates will turn out as average values. As the periodicity is increased, the actual pump speeds will more and more follow the set values.

To characterize the realized outcome of the zig-zag pump profiles, calibration measurements of the fluorescence intensity of fluorescein are taken into account. For these experiments the aqueous component aq 1 is a $100 \mu \mathrm{M}$ fluorescein solution and aq 2 as well as aq 3 are water. First, the fluorescein concentration is set to a constant value for a long time. This way no inertial effects of the system are present and the fluorescein concentration in the drops can be calculated. The comparison of these calculated fluorescein concentrations to the mean fluorescence intensity in the drops shows a linear dependence (Fig. 6.1a). In experiments with the periodic zig-zag pump rates, the concentration of fluorescein in the drops is directly calculated on the basis of the input flow rates. Additionally, the concentration can be calculated from the mean fluorescence intensity in

a)

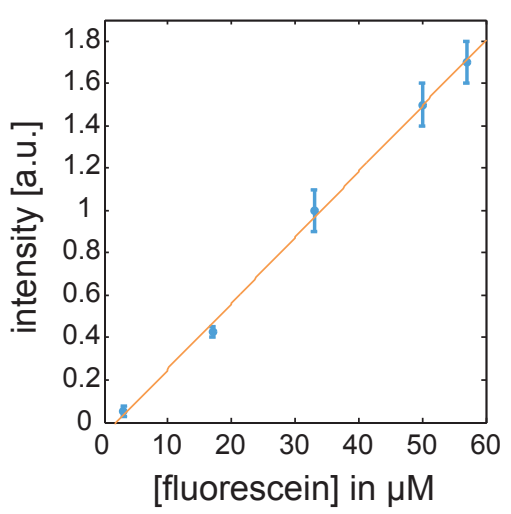

b)

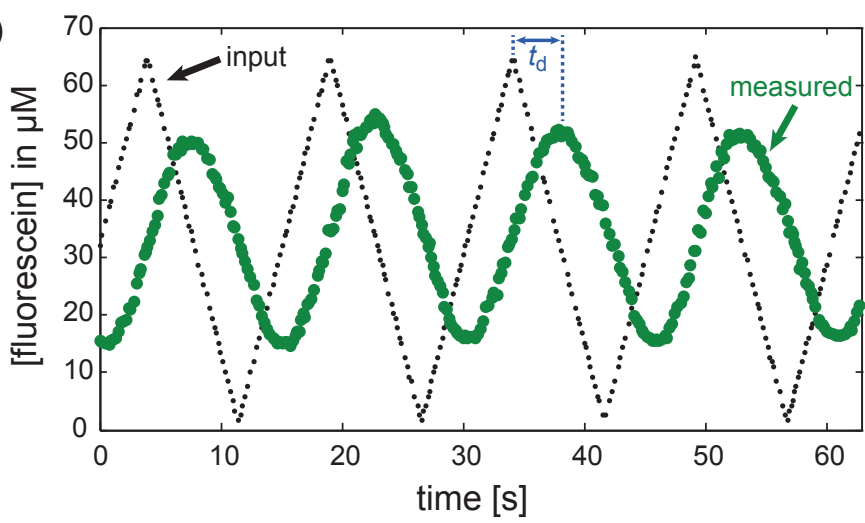

Figure 6.1: Fluorescein concentration in drops. a) At constant pump rates, the fluorescence intensity shows a linear dependence (fit) on the fluorescein concentration. The intensity at $33 \mu \mathrm{M}$ fluorescein is set to 1 . b) The input pump rates follow a zig-zag course with a periodicity of $15 \mathrm{~s}$. From these input rates, the calculated fluorescein concentration is given (black). These calculated concentrations are compared to measured concentrations (green). The resulting concentration profile has the shape of a sine-function and appears retarded by the time $t_{\mathrm{d}}$. 
the whole drop (Fig. 6.1b). The measured fluorescein concentration shows the aforementioned retardation by the delay time $t_{\mathrm{d}}$ and the sine-shape of the concentration profile in comparison to the calculated profile.

The quantity of interest is the actual concentration of fluorescein $c_{\mathrm{Fl}}$ in the drops, or the magnesium chloride concentration in the actual experiment with vimentin. This concentration is a function of the time $t_{k}$, when the $k$ th drop in the drop series is collected (Fig. 4.2). Considering the delay time, the concentration in the $k$ th drop is then [34]:

$$
c\left(t_{k}\right)=\frac{c_{\mathrm{syr}}}{Q_{\mathrm{aq}}} \times Q\left(t_{k}-t_{\mathrm{d}}\right),
$$

where $c_{\mathrm{syr}}$ is the concentration of fluorescein (or magnesium chloride) in the syringe, $Q_{\mathrm{aq}}$ the overall flow rate of the three aqueous components and $Q\left(t_{k}-t_{\mathrm{d}}\right)$ the flow rate with which the $k$ th drop is produced. The flow rate profile $Q(t)$ is measured on the basis of eight measurements of fluorescence intensity in the drops as shown exemplary by the green curve in Fig. 6.1b. A fit gives the amplitude and the offset in the resulting flow rate:

$$
Q(t)=(5.2 \pm 0.1) \mu \mathrm{L} / \mathrm{h} \times \sin \left(\frac{2 \pi}{15 \mathrm{~s}} t-\phi_{d}\right)+(10.2 \pm 0.1) \mu \mathrm{L} / \mathrm{h} .
$$

This corresponds to a zig-zag flow profile of $15 \mathrm{~s}$ periodicity ranging from 0 to $20 \mu \mathrm{L} / \mathrm{h}$ at maximum for aq 1 and $Q_{\mathrm{aq}}=30 \mu \mathrm{L} / \mathrm{h}$. When the first drop of the series is collected (Fig. 4.2), we set $t=t_{k}=0$. The phase $\phi_{d}$ is a parameter of each experiment and considered in the determination of the delay time. However, the first drop is not composed at the flow rate $Q(t=0)$. Considering the delay time $t_{\mathrm{d}}$, it is produced some time earlier, i.e. $Q\left(0 \mathrm{~s}-t_{\mathrm{d}}\right)$. The same holds for the $k$ th drop, and that is why Eq. 6.1 has the given form.

The delay time is in the range between 3-5 s. We determine its exact value for each experiment with a regression method, which will be explained on the basis of the following example. In Fig. 6.2, we give the number of collected drops as a function of time and the measured fluorescein concentration $c_{\mathrm{m}}$ that is determined by the fluorescence intensity in the drops. The fluorescence intensity is the first method to determine the fluorescein concentration in each drop. For the second way, we use Eq. 6.1 and therefore determine the concentration with the knowledge of the flow rates that the drops are filled with. For this purpose, an arbitrary delay time $t_{\mathrm{d}} \in[0,14.9 \mathrm{~s}]$ is chosen. This way a fluorescein concentra- 
a)

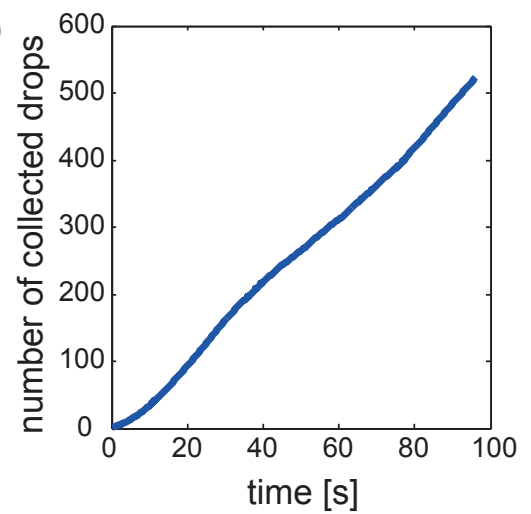

b)

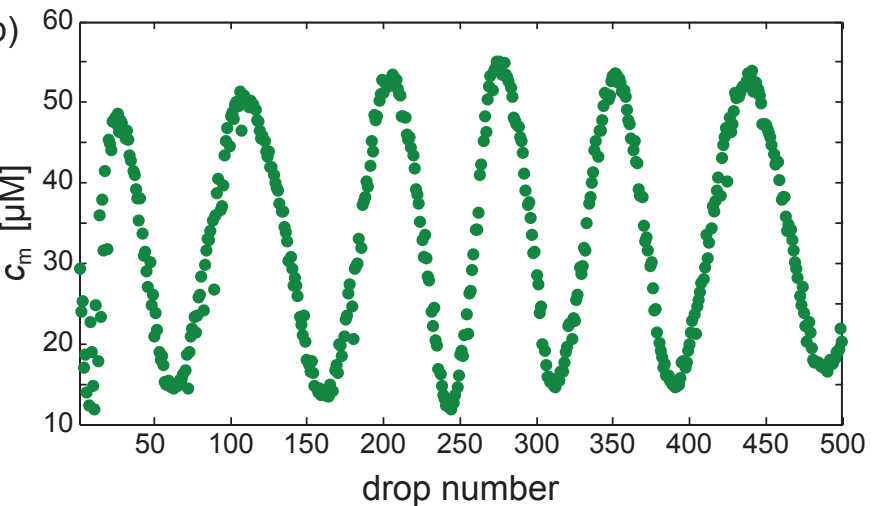

Figure 6.2: Properties of drops during the collection process. Example for concentration determination for fluorescein in drops. a) Number of collected drops. b) Fluorescein concentration in the drops based on the fluorescence determination in each drop.

tion $c_{\mathrm{Fl}}$ for each drop is calculated (examples: Fig 6.3a/c/e).

The content in each drop has to be characterized by a single scalar ('signal'). The signal in this example is the intensity in each drop, which is equivalent to the measured fluorescein concentration $c_{\mathrm{m}}$. For the experiments with vimentin the signal is a scalar that characterizes protein aggregation. In Fig. 6.3a, c and e, the calculated concentration $c_{\mathrm{Fl}}$ is shifted to the signal $c_{\mathrm{m}}$ owing to different values of $t_{\mathrm{d}}$. The $c_{\mathrm{m}}$ value is a parameter of each drop. However, the assigned $c_{\mathrm{Fl}}$ value changes with the choice of $t_{\mathrm{d}}$. For a given $t_{\mathrm{d}}$, the $c_{\mathrm{m}}$ value of each drop is plotted against the $c_{\mathrm{Fl}}$ value of each drop in $\mathrm{b}, \mathrm{d}$, and $\mathrm{f}$. Interestingly, the resulting graphs are Lissajous curves, as in the figure the data points are scattered following sinelike functions for the $x$ and $y$ coordinates. [84] It will be necessary to group drops of similar concentration $c_{\mathrm{Fl}}(3 \mu \mathrm{M}$ concentration bin width). In our example experiment, the delay time is $t_{\mathrm{d}}=5 \mathrm{~s}$. In $\mathrm{b}$ it can be seen that for this delay time, the data points in each group show the least scattering for the same concentration $c_{\mathrm{Fl}}$ in comparison to $\mathrm{d}$ and $\mathrm{f}$. This relation is used to actually determine $t_{\mathrm{d}}$. The average $\bar{c}_{\mathrm{m}, i}$ together with its error $\delta_{i}$ is considered for each group. A 'penalty function' $E$ is introduced as the sum over all these errors:

$$
E:=\sum_{i} \delta_{i}
$$

Since the grouping of the drops into classes depends on the delay time $t_{\mathrm{d}}, E$ is a function of $t_{\mathrm{d}}$. Like in Fig. 6.3b, the deviation of the data points for each bin will be much smaller, when 'signal' $c_{\mathrm{m}}$ and $c_{\mathrm{Fl}}$ lie on top of each other. Therefore, min- 
a)

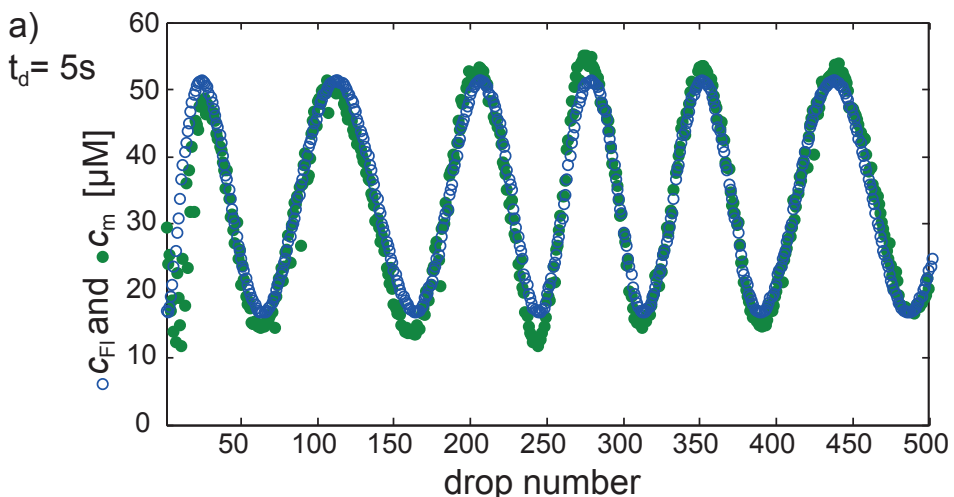

c)

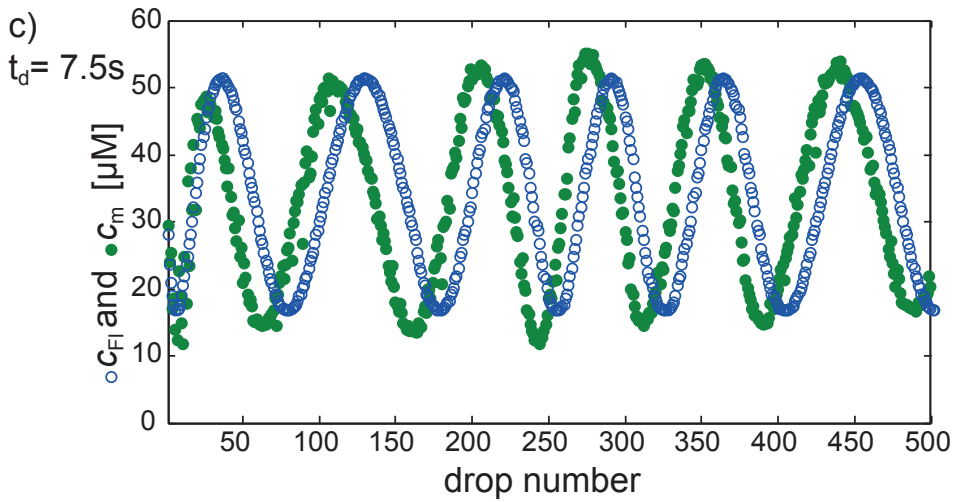

e)

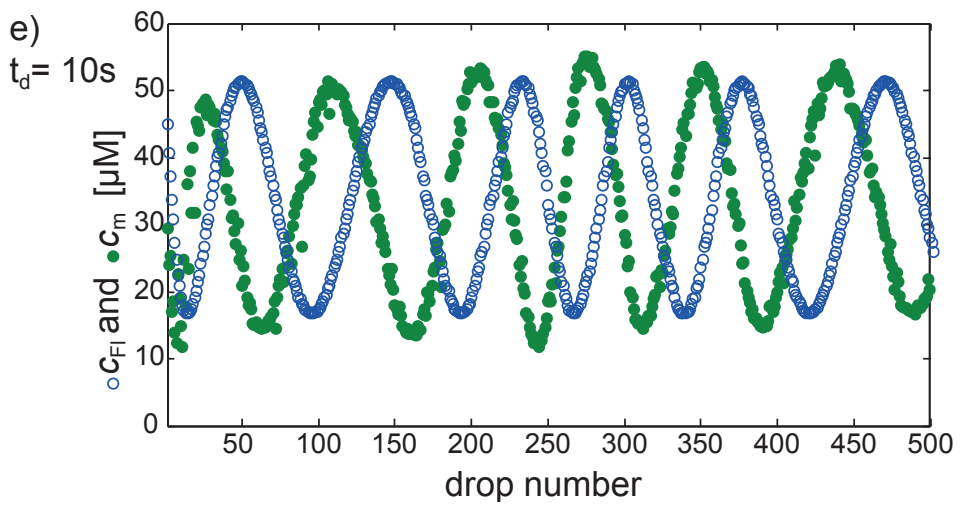

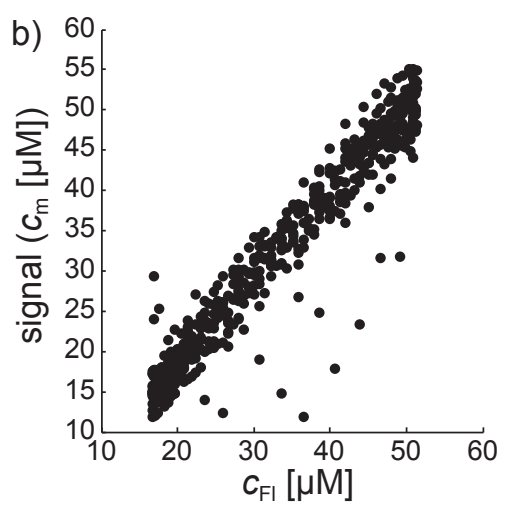
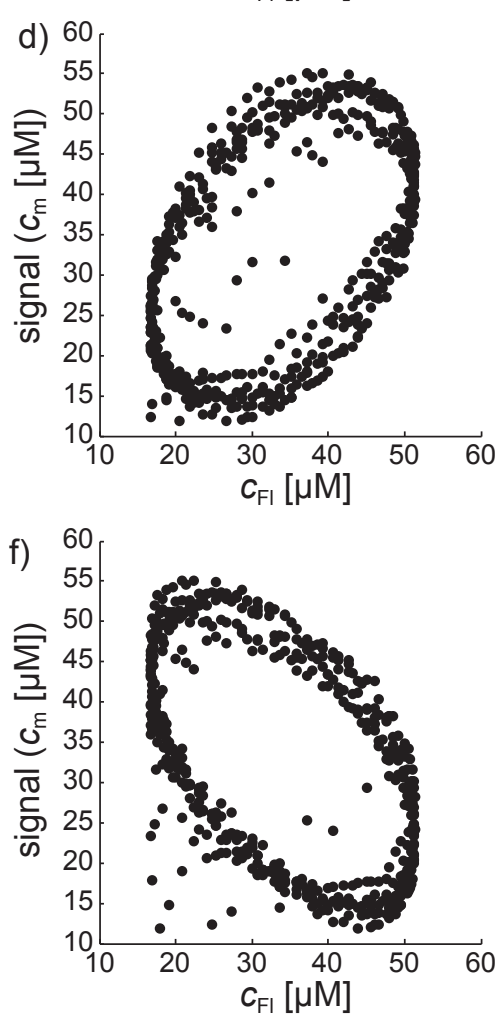

Figure 6.3: Meaning of the delay time for drop grouping. In a,c,e different example delay times $t_{\mathrm{d}}$ determine the shift between the calculated fluorescein concentration $c_{\mathrm{Fl}}$ and the measured concentration $c_{\mathrm{m}}$ ('signal'). For a given time $t_{\mathrm{d}}, c_{\mathrm{m}}$ is plotted as a function of $c_{\mathrm{Fl}}$. The result are Lissajous curves (b,d,f). The concept of testing different delay times as shown for the three examples in this image is used to determine the actual delay time (see Fig. 6.4).

imization of $E$ with respect to $t_{\mathrm{d}}$ results in the best fit. In Fig. 6.4a, $E$ is given with respect to $t_{\mathrm{d}}$. Two clear minima are found in this curve. The first minimum at $t_{\mathrm{d}}$ is the relevant one. The second one would correspond to the non-physical case of maximum fluorescence at the lowest fluorescein concentration and is therefore meaningless. Using this delay we give the averaged intensity $\bar{c}_{\mathrm{m}, i}$ of each concen- 
a)

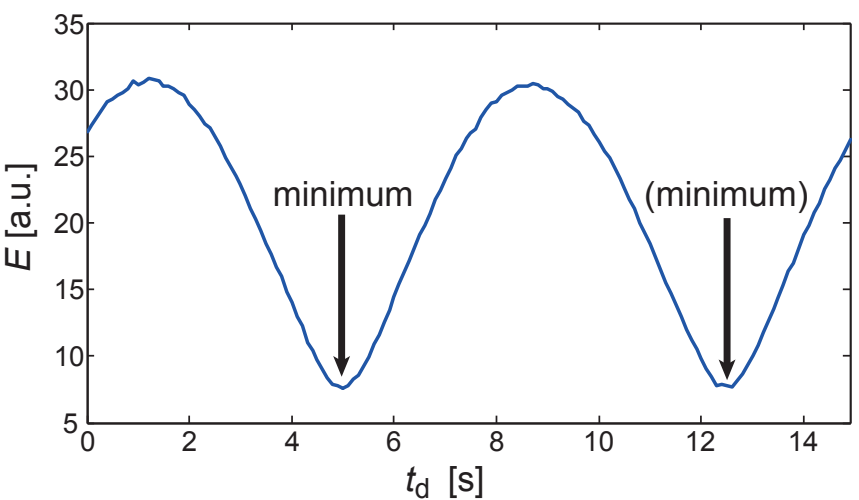

b)

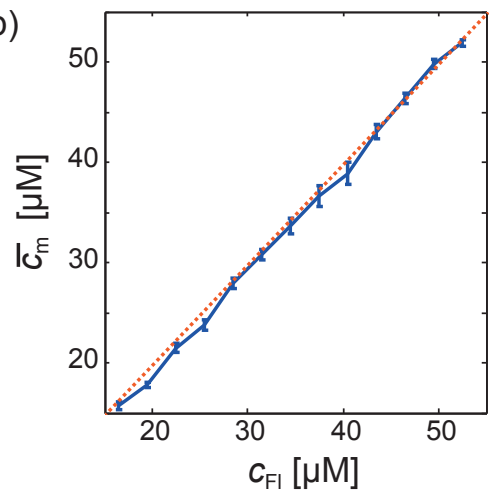

Figure 6.4: Extremal principle for the determination of the delay time. a) Minimization of the $E$ value (Eq. 6.3) determines the best fitting delay time to $t_{\mathrm{d}}=5 \mathrm{~s}$. Since the approximate delay time is $3-5 \mathrm{~s}$, the second minimum can be neglected. It would correspond to the non-physical case of decreasing fluorescence intensity with increasing fluorescein concentration. b) The resulting average for each concentration group is given. The straight line has a slope of 1 .

tration group in Fig. 6.4b. The data follow nicely a straight line with a slope of 1. Of course, in this case this is what we expect, as we determine the fluorescein concentration using two methods. This example shows the high precision of the determination of the content in the drops. In Sec. 7.1, we will use this method to determine the magnesium chloride amount in each drop, which is not directly accessible the way the fluorescence intensity is in this case. The morphologies of the vimentin filament networks are used as a 'signal' and they are represented by a scalar. There the algorithm as explained here is needed and will reveal the otherwise unknown magnesium chloride concentrations.

In general, the algorithm is also applicable to other systems. The only requirement is that the 'signal' measured in the drop has a monotonic dependence on the chemical that is changed from drop to drop and it is of scalar form.

\subsection{Network morphologies in time-lapse studies}

\subsubsection{From raw data to network morphologies}

In the time-lapse studies with the device $\mathrm{T}$, vimentin filament aggregates were imaged with a confocal microscope (Sec. 3.4). The scanning of the images has to be done quickly, as the network of filaments changes continuously. At the same time, the laser intensity for the excitation of the fluorophores has to be moderate, 
a)

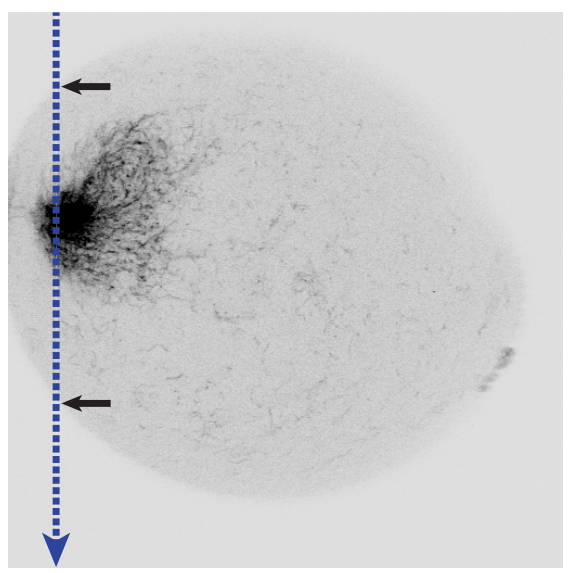

b)

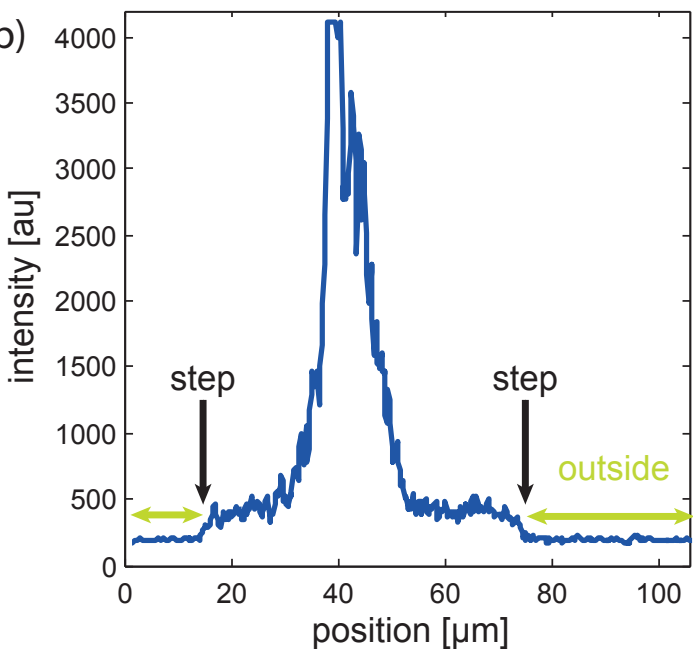

Figure 6.5: Fluorescence intensity regions in the drops. The intensity profile along the line in (a) is given in image (b). The images are divided into three regions: The region outside the drop, the background region in the drop as well as the signal region in the drop. The black arrows indicate the 'step' in intensity which is used to determine the drop outline.

to keep photo bleaching of the Alexa 488 dye as small as possible. Within these limits, the fluorescence of the labeled filaments is often only slightly above the noise level from the background. In Fig. 6.5, an example is given. In this example three regions can be identified. The first region is the region outside of the drops. It can be clearly distinguished from the drop region (second region), as there is always a step in fluorescence intensity (Fig. 6.5b). Consequently, the background signal in the drops cannot only originate from noise from the detector, as the region outside the drop has a smaller background signal. It is most likely due to small labeled vimentin filament precursors (Sec. 2.1.3). The third region consists of pixels that show fluorescence signal from vimentin filaments. Within the drops dense filament clusters are detected easily (peak region in b). However, next to these clusters there are filaments or small filament clusters, which have a fluorescence signal only slightly above the noise level. In the analysis of the whole image stack, it is also a problem that photo bleaching changes the absolute intensities over time. [71]

Therefore, the data require an algorithm that accounts for this type of noisy images. A key challenge during analysis is the decision whether a pixel in the image belongs to a filament or background noise. 


\subsubsection{Binarization of images}

The simplest method to discriminate between pixels in the data image that show noise and pixels that show signal is thresholding by intensity. This way the signal is best identified when the signal is considerably higher than the noise, i.e. the signal-to-noise ratio is large. However, thresholding gets worse as soon as the signal intensity values are only slightly above the noise level. When thresholding the image in the latter case, two types of mistakes are made: Let $\Psi$ be the intensity threshold and $I(m, n)$ the intensity of a pixel at position $(m, n)$. The first type of error is made when a pixel with $I>\Psi$ is detected that is noise but treated as part of the signal. The second type of error is made for pixels with $I<\Psi$ that belong actually to the signal but are treated as noise. In this section we will discuss how to avoid these two types of error by the consideration of side conditions for the signal during image binarization.

Image analysis is performed with MATLAB ${ }^{\circledR}$ (MathWorks ${ }^{\circledR}$, Natick, USA). The confocal images of aggregating vimentin are recorded with a typical frame-toframe time of $1.6 \mathrm{~s}, 207 \mathrm{~nm}$ pixel size and a dwell time of $4 \mu \mathrm{s}$ for each pixel of the $512 \times 512 \mathrm{px}^{2}$ images. Due to the dynamical range of the photo multiplier of the confocal microscope, intensities take a value in the range of 0 to 4095 (12 bit) for each pixel. First, a Gaussian filter is applied to each image (region $3 \times 3 \mathrm{px}$, standard deviation 0.5). This levels out noise a bit, while the details of the networks are preserved. Ten reference regions, which show background only, are selected in the whole image series (Fig. 6.6a). These background regions are stable to bleaching throughout the image series, which is due to the material exchange of the small molecules with off-focus regions. From the intensity analysis in the reference regions an average 'fingerprint' of the background is determined. A histogram of this average is given in Fig. 6.6b. The fingerprint follows a Gaussian distribution quite well. However, there is no need to perform a fit here, since the fingerprint is used directly in the following steps. For each frame of the image sequence, it is then decided for each pixel whether it contains 'signal' from a filament or background. The following steps are explained for the example image in Fig. 6.6c.

The intensity distribution for the total drop is shown in Fig. 6.6d. In this histogram, intensity within a bin width of 10 have been grouped. A first glance on this histogram also mirrors the challenge in data analysis: There is only one visible peak. This means that background and signal pixel have a strong overlap. 
a)

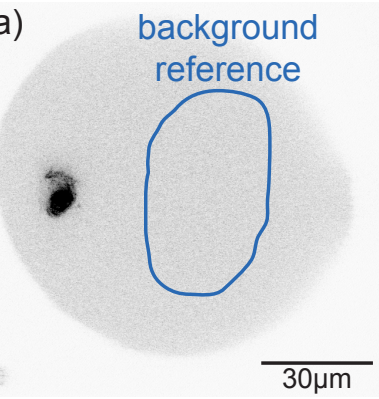

A B b)

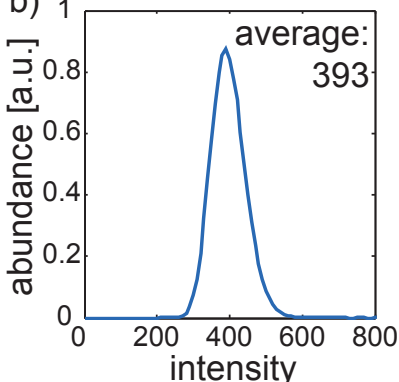

C d)

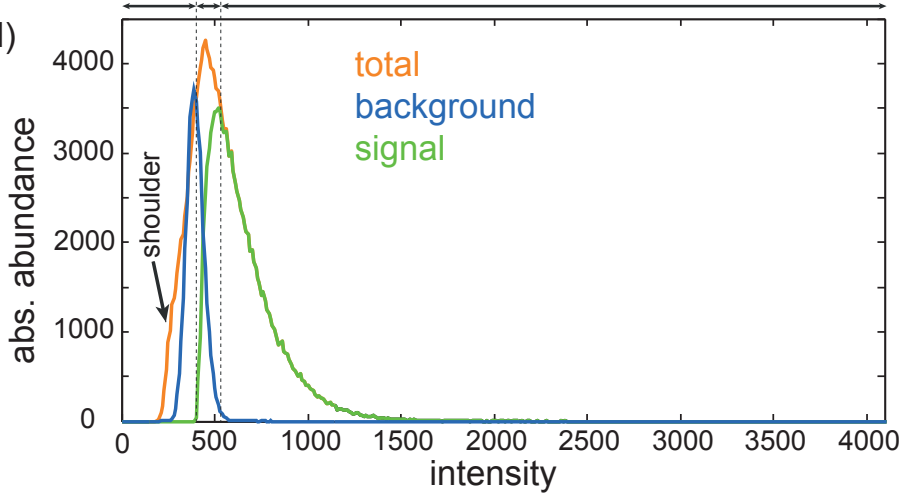

c)
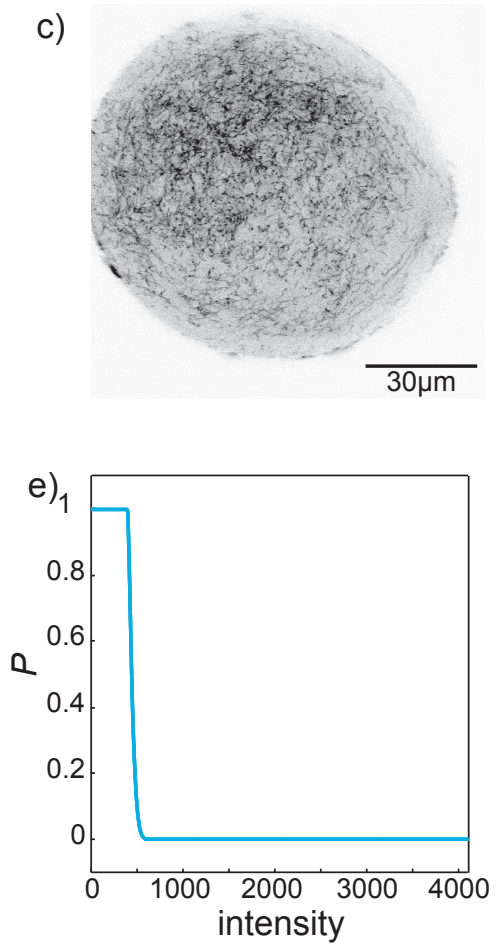

Figure 6.6: Investigation of image intensity details for background detection. a) An example of a reference region for background pixels. Several of these reference regions in the time-lapse image stack are taken to get the fingerprint of the background intensities (b). d) The total intensity histogram of an example image (c) is analyzed for the contribution of the background and the 'signal' (= vimentin filaments). e) For each intensity in the image, a probability $P$ is assigned. $P(I)$ gives the probability to detect a randomly chosen pixel with intensity $I$ as signal, although it belongs to the background. (Subfigure a) is adapted from reference [35] with permission from The Royal Society of Chemistry)

Analysis would be much easier, if there were two distinct peaks, for background and signal each. The fingerprint of the background is scaled up to the histogram of the total drop, so that the difference of the total histogram and that of the background is minimal in the range from 0 to an intensity of 393, which is the average of the background. This step is motivated by the reasonable assumption that below the mean background value, the histogram of the intensity in the total drop is composed of background pixels only. Notably, in the histogram of the total drop a shoulder is found at the left side of the background histogram. This shoulder is due to background pixels found at the edge of the drops and can be neglected (see also 'steps' in Fig. 6.5). When the background histogram is subtracted from the total histogram, the resulting histogram represents the histogram of the signal, i.e. intensity that arises from the fluorescence of filaments. In this signal curve, 
any intensities smaller than the background average are set to zero. The analysis of the composition of the histogram leads to three different regions of intensity: A, B and C (Fig. 6.6d). If a pixel has a low intensity in region A, which reaches from 1 to the background average 393, it is part of the background signal. From a certain intensity value, all pixels show signal intensity (start of $\mathrm{C}$ ). Between these two regions, for each pixel there is a certain probability that a pixel belongs to the signal or the noise (region $\mathrm{B}$ ). Consider the random variable $P$, which gives

a)

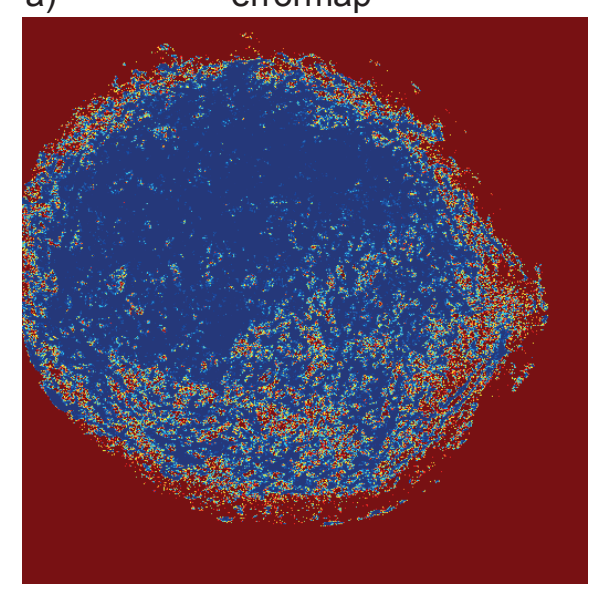

c)

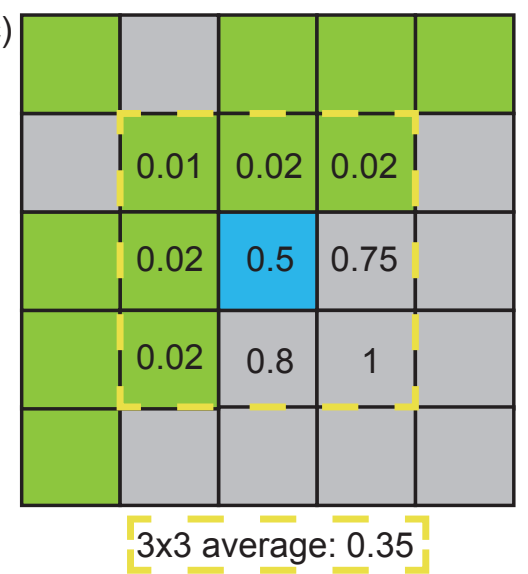

b) $\quad 3 \times 3$ average errormap
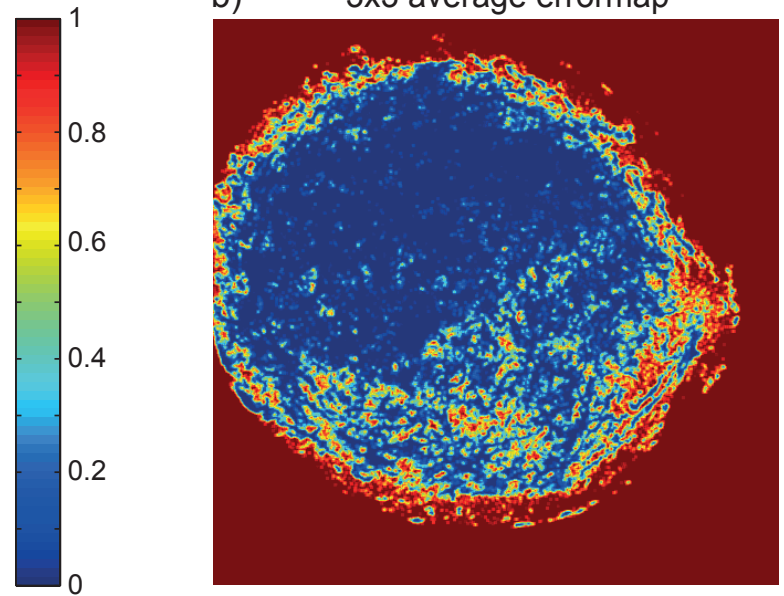

d)

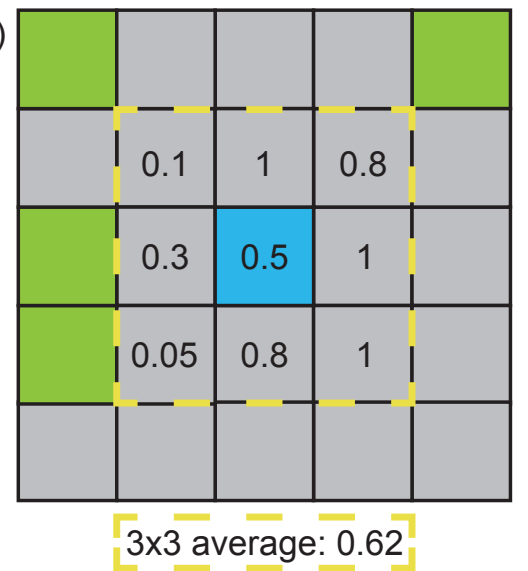

Figure 6.7: Errors for detecting background as signal. a/b) Errormaps: Probability to make an error, when a pixel is treated as filament/signal. In b) this error is averaged over a $3 \times 3$ neighborhood. In c) and d) the basic idea of the interpretation of the errormaps is illustrated. The question is whether the central (blue) pixel shows the signal of a filament or not. In c) and d) the blue pixel shall have the same intensities $I_{\mathrm{b}}$, and therefore the same error of $P\left(I_{\mathrm{b}}\right)=0.5$. As the pixel in $\mathrm{b}$ ) is close to a filament, its neighboring pixels have a high intensity and therefore a lower error probability $P$ compared to the neighborhood in d), where the central pixel is surrounded by background pixels. Accordingly, the resulting $3 \times 3$ average gives a lower error probability for the pixel distribution illustrated in c). 
the probability of the following event: When a pixel is chosen randomly in the drop, how large is the error probability, when this pixel is treated as signal? An error is made, when the pixel belongs to the background, but is treated as part of a filament. Based on the histograms we give the value of $P$ as a function of intensity $I$ of a pixel by (Fig. 6.6e)

$$
P(I)=1-\frac{\text { abundance of } I \text { in signal histogram }}{\text { abundance of } I \text { in total histogram }} \text {. }
$$

The region in the curve of $P$ where it is not 1 or 0 is quite narrow (Fig. 6.6e). However, it is also important as many of the pixels have their intensity in this region (compare region $B$ in Fig. 6.6d). Based on this error probability $P$ each pixel is assigned an error probability, leading to 'errormaps' for a whole image (Fig. 6.7a). Additionally, the errormap is averaged over its $3 \times 3$ neighborhood (b). These errormaps help to distinguish two pixels that have the same intensity. At first, they cannot be distinguished because of the equality of their intensity. However, when the neighborhood pixels are taken into account, a quantitative criterion makes the pixels distinguishable. This principle is demonstrated in Fig. $6.7 \mathrm{c} / \mathrm{d}$ in which the averaged error probability is lower in the neighborhood of a filament. It is reasonable to accept these pixels as signal rather than pixels which have background around them.

For the whole image, all pixels with the same intensity are analyzed simultaneously. Pixels are accepted as signal starting with the pixel that have the lowest $3 \times 3$ averaged error probability. Subsequently, pixels with a higher $3 \times 3$ averaged error probability are accepted. The acceptance of a pixel as signal is stopped, when the number of accepted pixels equals the number of pixels that actually build the signal histogram (Fig. 6.6d). The resulting binary image (1: signal/filament, 0: background) is in conformance with Eq. 6.4. In a final step, small isolated clusters are removed (less than 40 connected pixels), as these are too small to represent filaments.

In Fig. 6.8, the outcome of the analysis of a time-lapse image stack is given exemplary. In a) and b) it can be seen that this method neglects small regions of background, where the filaments are dense. Instead, only regions of large background are detected as background regions. Therefore, the method detects clusters of pixels best. The key elements of this detection are the fingerprint of the background and the preference to detect image clusters instead of considering a pixel individ- 


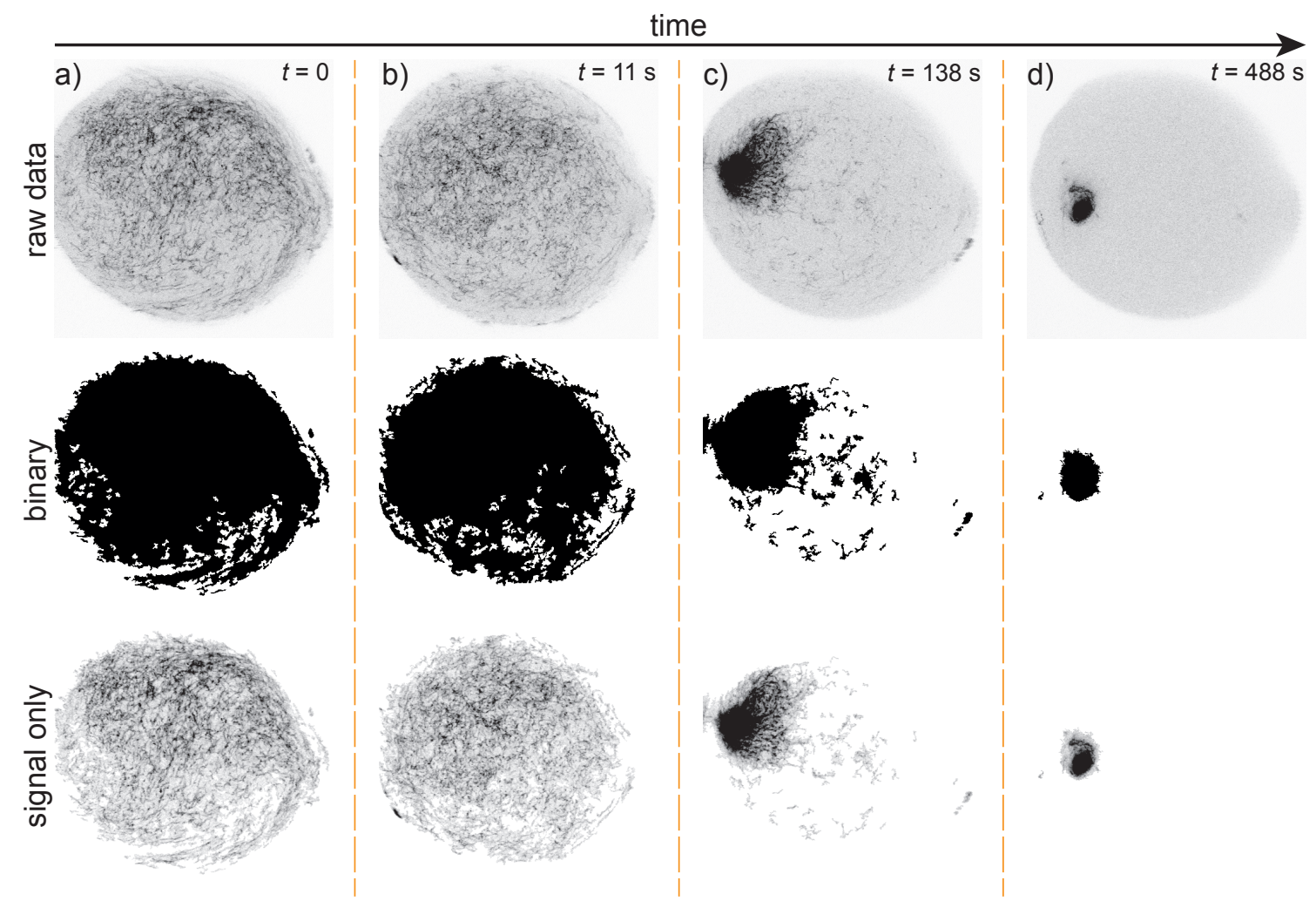

Figure 6.8: Binarization of images. In a time-lapse series, the raw image data are compared to the binary form (black: pixel detected as 'signal', white: background). In the lower row, only the signal is given (raw data without background pixel). The image side lengths are $106 \mu \mathrm{m}$. (Partly adapted from reference [35] with permission from The Royal Society of Chemistry)

ually. The latter preference is reasonable as the fluorescence of micrometer-sized filaments should lead to fluorescence clusters and not to an isolated fluorescence pixel.

An alternative to the method presented here are auto-thresholding methods, which can provide a good binary image of the networks in some cases. However, a threshold is a fixed value for each image. For instance, two pixels of equal intensity like in Fig. 6.7 would be treated the same, although they do not represent the same. Additionally, these methods do not work for all of our datasets. Especially in cases where the overall signal of the filaments is weak compared to the background and where bleaching effects increase over time, these auto-thresholding methods have shown to be not reliable for our data. In contrast, our method is stable for entire time-lapse stacks of several hundred images in virtually all cases. At the same time, highly bleached filaments are still detected to a large extent. 


\subsubsection{The convex hull of networks}

We carried out time-lapse studies with the device $\mathrm{T}$ using the confocal microscope. The resulting images provide details on the networks of vimentin filaments (Fig. 6.8). The challenge is to quantify the different morphologies. In this analysis many factors play a role. Most importantly, bleaching effects occur due to repeated imaging of the same filaments. Therefore, analysis methods that sensitively depend on absolute intensity are not suitable.

For instance, the absolute value of the standard deviation depends on the overall intensity in the drop. For the measurements in the device $C$, this is not an issue, as the networks are imaged once and bleaching does not play a role (Fig. 7.1). The area of the binarized images is also in principle a measure for the expansion of the network in the drops. However, this method is highly influenced by bleaching. For example, as the visible diameter of a bleached filament shrinks over time. Additionally, this parameter depends also on the imaging details, like the step size of the pixels in a scan. There are other methods that account for the mesh sizes or measures for the inter-filament distances in networks. For example, 'bubbles' are fitted into meshes giving an estimate of the mesh size of the networks. [86,89] Statistics on the filament to filament distance are considered. $[9,62,63]$ However, our networks are in most cases so dense that most of the details of the meshes are simply not resolved, and cannot be analyzed with these methods.

One distinct property of the networks is their pronounced compaction over time (Fig. 6.8, from a to d). Within this compaction the area of an envelope to the whole networks gets smaller over time. For a clear quantification of the area of such an envelope, the convex hull ${ }^{1}$ is considered. This quantification has two advantages: First, the convex hull has a clear mathematical definition and is therefore unique for each binary image. Second, the way the raw data is converted to binary images (Sec. 6.2.2) and therefore prepared for the convex hull analysis, is much more robust against bleaching effects compared to the above mentioned techniques. The convex hull for a binary image is found by the usage of the MATLAB function 'regionprops', which is fast and straightforward to use.

In Fig. 6.9, the examples of convex hulls are shown. In the first row, it becomes obvious that, if the convex hull is taken for the total binary image, it is dispro-

\footnotetext{
${ }^{1}$ The convex hull of a binary image is the smallest set of pixels that contains all non-zero pixels as well as all pixels that are in between a straight line between two arbitrarily chosen non-zero pixel of the binary image.
} 


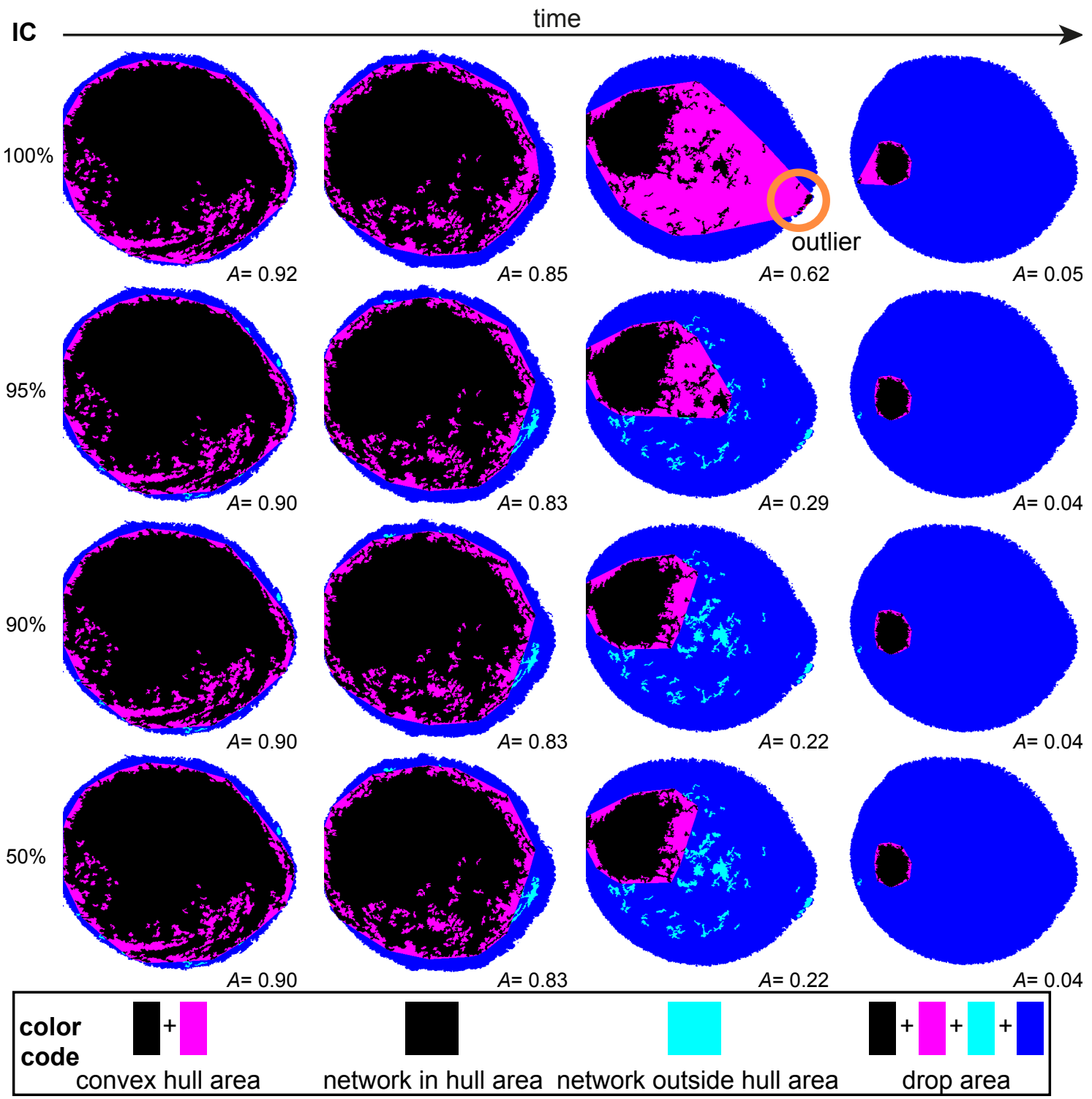

Figure 6.9: Convex hull of binary images. For the binary images from Fig. 6.8, the convex hull is given. In the different rows, the convex hull is chosen in a way that it covers at least IC $=100 \%, 95 \%, 90 \%$ or $50 \%$ of the total network intensity (IC: intensity conserved). For cases of, IC $=90 \%$ and lower, the calculated hulls are robust against outliers that distort the hulls disproportionally. Additionally, the relative drop hull area $A$ is given. (Partly adapted from reference [35] with permission from The Royal Society of Chemistry)

portionately influenced by outliers. To make the convex hull a robust quantity, the intensity of the network is taken into account. The binary images consist of one or more connected regions ('islands'). For the $i$ th island, the intensity in the corresponding region of the raw data image is summed up to a value $S_{i}$. The total intensity $S$ of the network is then the sum over all $S_{i}$. Starting with the is- 
lands with the smallest $S_{i}$, the islands are excluded from the convex hull until a certain fraction IC of total intensity in the drop is still conserved by the remaining islands. For example, for IC $=90 \%$, small islands are removed until at least $90 \%$ of the total intensity is still there. This concept leads to different outcomes of the convex hulls (Fig. 6.9). It is found that IC $=90 \%$ is a value, at which most of the low intensity outliers are excluded from analysis, while the characteristic part of the network is enclosed in the convex hull. For lower values than IC $=90 \%$, the hull does typically not change, as the largest part of the intensity is in one big island.

The area of the convex hull (IC =90\%) characterizes the expansion of the characteristic part of the network in the drop. To compare between drops of different sizes, it is reasonable to normalize this area to the area of the drop, leading to the relative hull area $A$ (Fig. 6.9). For instance, when two drops of different sizes have a network that is spread everywhere in the drop, the networks should be characterized by the same quantitative value. The relative hull area $A$ is then the same for both networks, although the absolute hull area is not. 



\section{7 \\ Interaction of vimentin and multivalent ions}

\subsection{Concentration dependence}

The device $\mathrm{C}$ is used to study the dependence of vimentin filament aggregation on the magnesium ion concentration (Sec. 4.1). We have published the results in Biomicrofluidics. [36] Fig. 7.1 shows vimentin networks at different magnesium ion concentrations $c_{\mathrm{Mg}^{2+}}$. The three examples show that magnesium ions mediate an aggregation of the vimentin filaments in the drops. In direct comparison of the drop with $c_{\mathrm{Mg}^{2+}}=6.5 \mathrm{mM}$ to the other higher concentrations, there is a qualitative difference. In the first case, the filaments spread over the whole drop and are freely fluctuating. In contrast, a network of filaments emerges at higher magnesium ion concentrations. Consequently, a threshold concentration is expected between $c_{\mathrm{Mg}^{2+}}=6.5 \mathrm{mM}$ and $12.5 \mathrm{mM}$. For a detailed investigation of the concentration dependence of this aggregation, we apply the algorithm as described in Sec. 6.1. Therefore, a scalar 'signal' from each vimentin network in the drops is needed to determine the exact magnesium ion concentration for each drop. We take the standard deviation $\sigma_{\mathrm{I}}$ of the intensity over the same area for each drop (orange circles in Fig. 7.1). Additionally, we normalize the total intensity for each drop to $10^{5}$ (a.u.), as $\sigma_{\mathrm{I}}$ depends on absolute intensities. This way, the standard deviations between different drops are comparable. For our examples in Fig. 7.1a-c, $\sigma_{\mathrm{I}}$ rises as the magnesium concentration rises. Consequently, the standard deviation fulfils the monotonicity criterion required (Sec. 6.1) and is a parameter 
a)
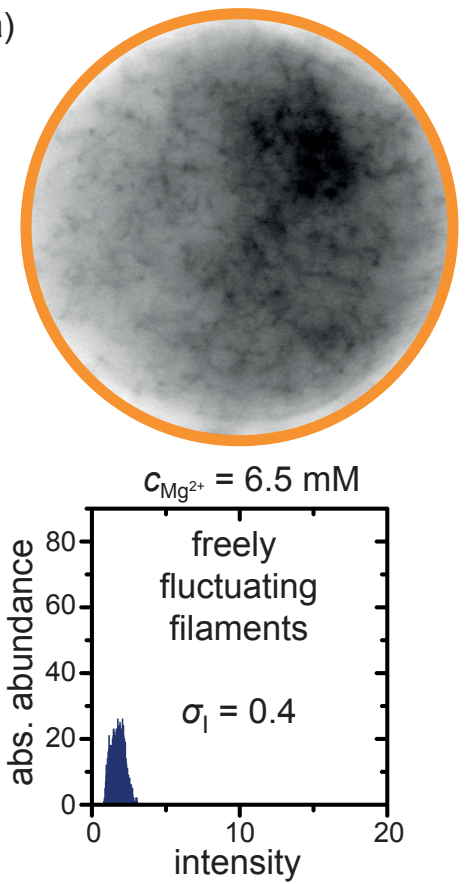

b)
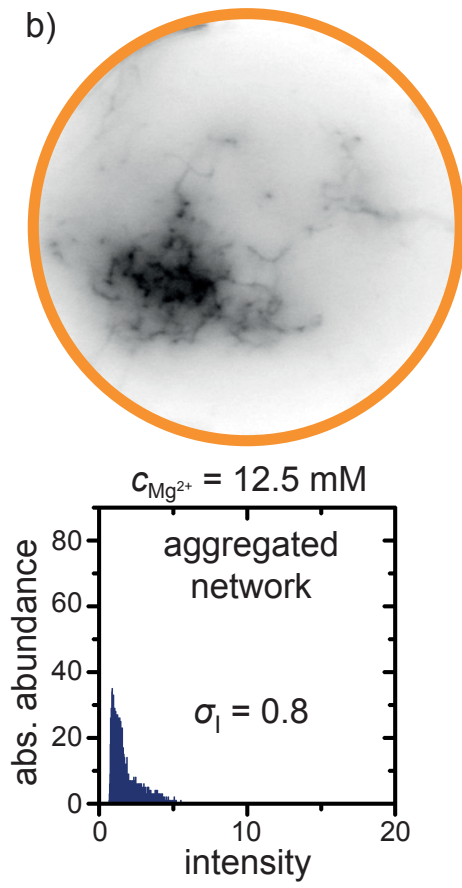

c)
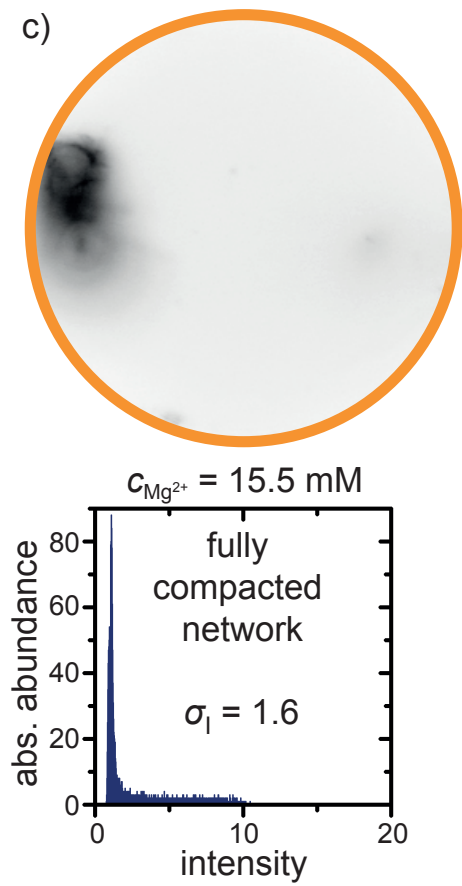

Figure 7.1: The standard deviation of the intensity quantifies network morphology. The figures show inverted grayscale fluorescence images of vimentin at different magnesium concentrations in the drop. The images are adjusted in brightness for displaying purposes. At higher magnesium concentration, network compaction increases. The standard deviation $\sigma_{\mathrm{I}}$ describes intensity variations within a whole drop (area within an orange circle, drop diameter $40 \mu \mathrm{m}$ ). For this purpose, the total intensity in the circle is normalized to obtain comparable $\sigma_{\text {I }}$ values. The standard deviation increases for higher magnesium concentrations. Therefore, $\sigma_{\mathrm{I}}$ is a parameter of aggregation. (Reused from reference [36] with permission from AIP Biomicrofluidics)

Figure 7.2 (facing page): Determination of drop compositions and drop content grouping. Vimentin filament aggregation in the presence of magnesium ions studied in the device $\mathrm{C}$ for two independent experiments $\mathrm{A}$ and $\mathrm{B}$. The data analysis is based on the method described in Sec. 6.1. The rate of the drop collection determines how many periods of the magnesium concentration the stored drops have (a and e). As the drop collection is faster in example B, about two magnesium periods can be investigated (g, open blue circles), whereas in A there are about four magnesium periods (c, open blue circles). The analysis of the $E$-value shows a clear first minimum and determines precisely the delay time ( $\mathrm{b}$ and $\mathrm{f}$ ). A comparison between the magnesium ion concentrations $c_{\mathrm{Mg}^{2+}}$ in the drops and $\sigma_{\mathrm{I}}(\mathrm{c} / \mathrm{g})$ shows a correlation between these two quantities: For high magnesium ion concentrations, $\sigma_{\mathrm{I}}$ is also high. We group the drops according to their concentration $c_{\mathrm{Mg}^{2+}}$ (bin width $=1 \mathrm{mM}$ ) in order to determine the average standard deviation $\overline{\sigma_{\mathrm{I}}}$ of each group $(\mathrm{d} / \mathrm{h})$. As a main result of the analysis, we find that the onset of aggregation is at about $c_{\mathrm{Mg}^{2+} \text {, onset }} \approx 10 \mathrm{mM}$ in both cases. 

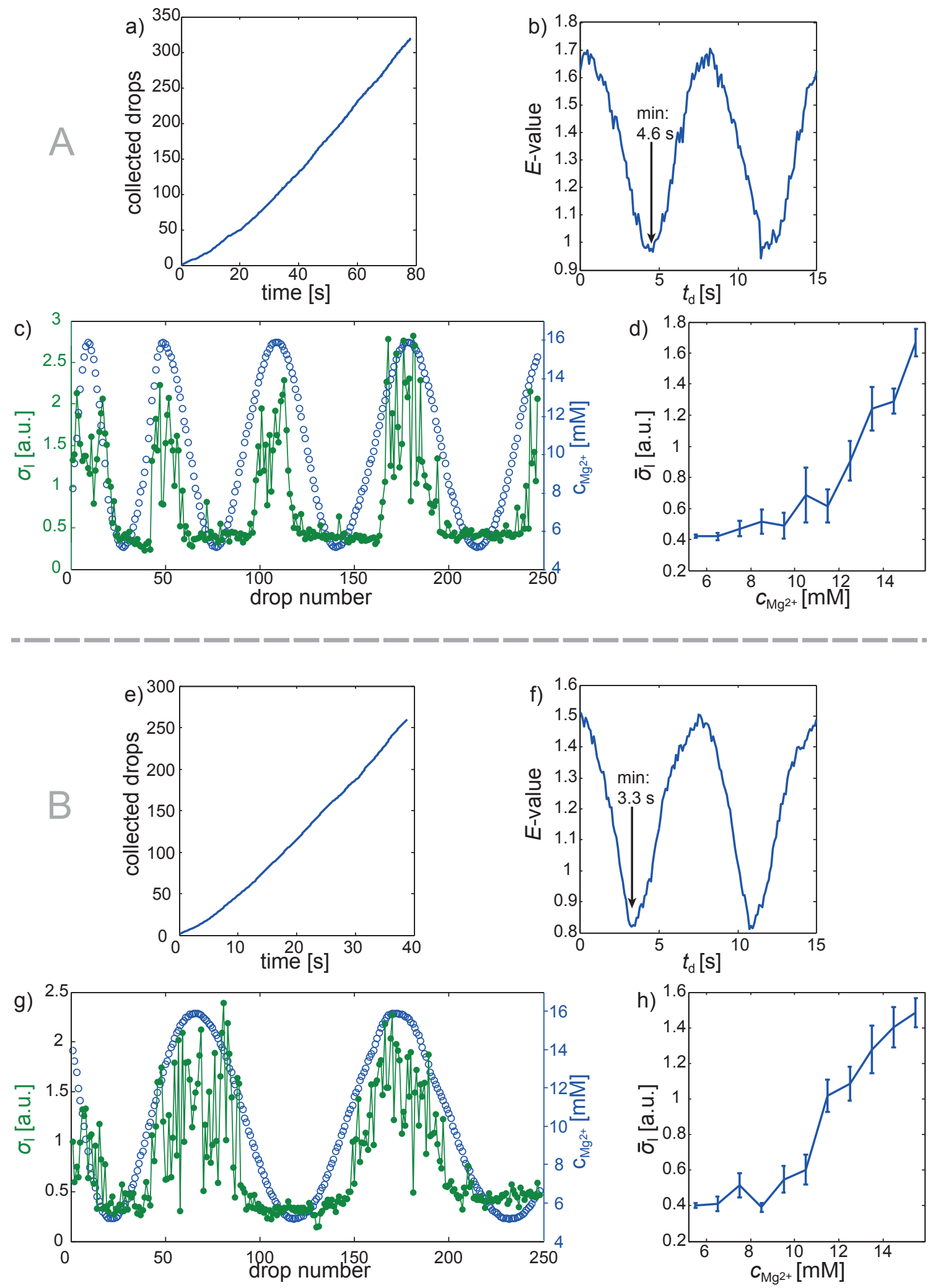
characterizing the aggregation. For two independent experiments, the analysis is documented in Fig. 7.2. The aggregation is characterized for each drop in the experiment by the $\sigma_{\mathrm{I}}$-value (c and $\mathrm{g}$, closed green circles). In analogy to the test experiments with drops that contained different concentrations of fluorescein (Sec. 6.1, Fig. 6.4), the $E$-values show a clear first minimum (Fig. 7.2b/f). This leads to a consistent match between the magnesium concentration and the

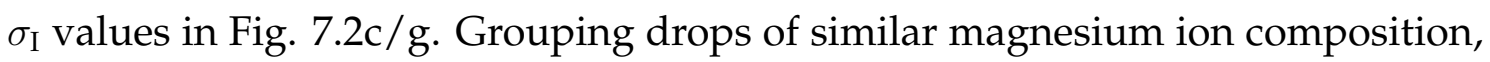
yields the dependence of vimentin filament aggregation as a function magnesium concentration. Based on plots in Fig. $7.2 \mathrm{~d} / \mathrm{h}$, the main findings are:

- The aggregation of vimentin filaments shows a monotonic increase with respect to the magnesium amount in the drops.

- There is a threshold magnesium concentration $\left(c_{\mathrm{Mg}^{2+} \text { onset }} \approx 10 \mathrm{mM}\right)$ upon which the aggregation of filaments sets in.

\subsection{Time-lapse studies}

We use the device $\mathrm{T}$ to investigate the aggregation behavior of vimentin in the presence of magnesium ions. We have published the results of this study in $L a b$ on a Chip. [35] For these experiments, two types of analysis methods are carried out. The first analysis is based on data from short and long dwell times of the drops in the traps (Sec. 4.2.3). These data are analyzed qualitatively only (Sec. 7.2.1), and show high consistency to the results obtained with the device $C$ (Sec. 7.1). In a second step, several of the datasets with long dwell times in the traps are then analyzed in detail (Sec. 7.2.2). 


\subsubsection{Qualitative aggregation analysis}

In the device $\mathrm{T}$, the drop dwell time in the traps follows a broad distribution (Sec. 4.2.3). This is due to the fact that drops can be squeezed through the small slit at the bottom of each trap (Fig. 4.5). Consequently, not all drops can be used to study the filament interaction over time in detail. However, there is also valuable information in drops that have a short dwell time in the traps (typically less than a minute) as aggregation to networks starts immediately after a drop arrives in a trap. In this analysis, we evaluate the interaction of filaments during any time the drops are in the traps. We distinguish filaments that show (first beginnings of) aggregation from those that show no aggregation. The example images in Fig. 7.3 illustrate that such a qualitative evaluation of the filament appearance is reasonable. The result of this analysis is given in Fig. 7.4. The restricted ob-
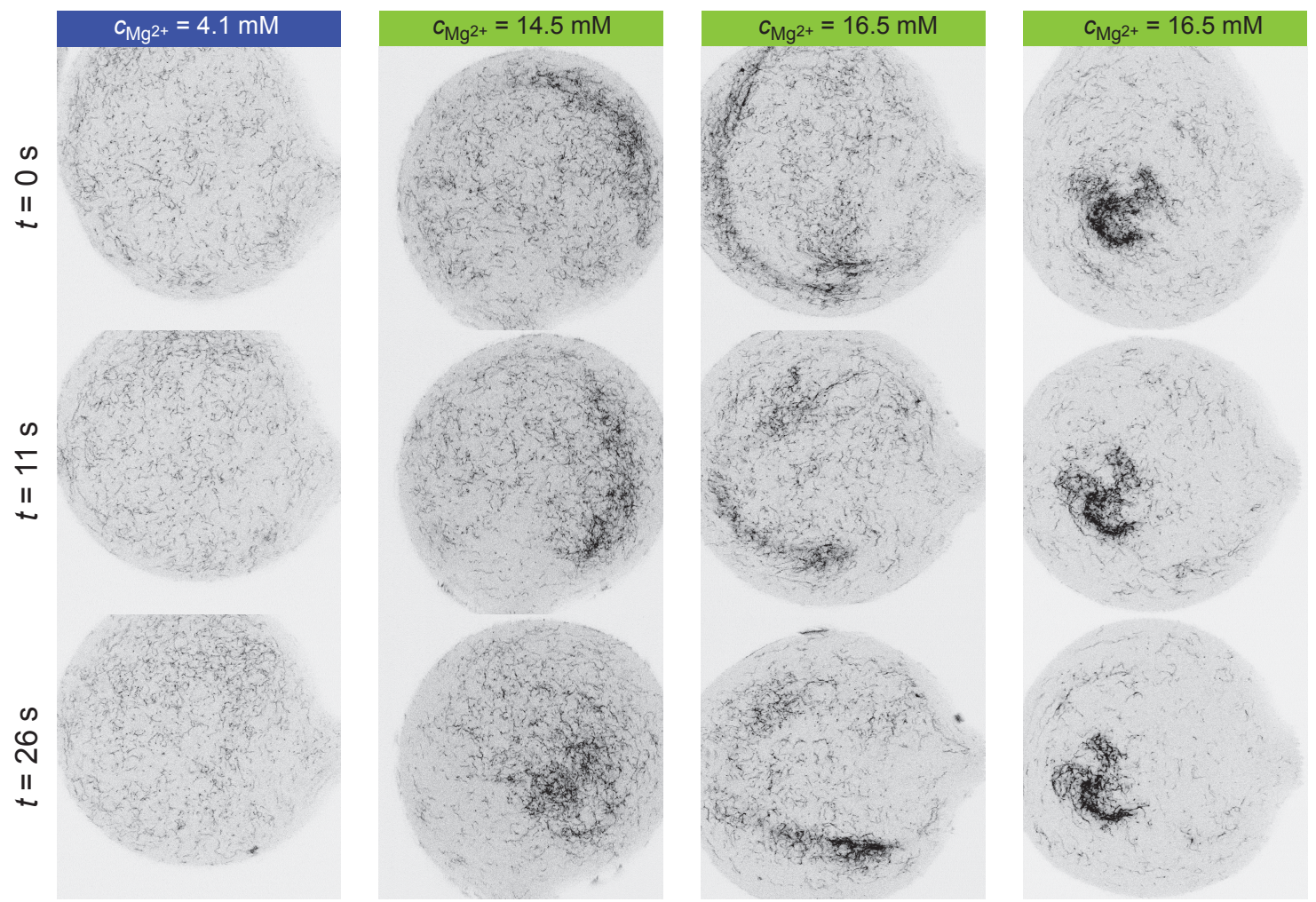

Figure 7.3: Vimentin aggregation observed for short trapping times. Already in the very first seconds after the drops arrive in the trap $(t=0 \mathrm{~s})$ it can be seen whether the vimentin filaments (inverted grayscale fluorescence) show aggregation (green, $c_{\mathrm{Mg}^{2+}}=$ $14.5 \mathrm{mM}$ or $16.5 \mathrm{mM}$ ) or not (blue, $c_{\mathrm{Mg}^{2+}}=4.1 \mathrm{mM}$ ). Domains of pronounced intensity, where the filaments are connected, are a clear indicator for aggregation. The image side length is $106 \mu \mathrm{m}$. 
servation time in this analysis raises the question whether an aggregation takes place after the drops left the traps. But with respect to the clear result of the long time observations in Sec. 7.2.2, we know that when aggregation occurs, then the filaments start to aggregate from the beginning on. This point is supported by the fast distribution of the small metal ions in the whole drop (Sec. 5.1). When the magnesium ions are everywhere at the beginning of the experiment, aggregation starts also at the beginning. The analysis of aggregation in the presence of magnesium chloride are shown Fig. $7.4 \mathrm{a} / \mathrm{b}$. The relative numbers in Fig. $7.4 \mathrm{~b}$ indicate a threshold concentration at $c_{\mathrm{Mg}^{2+}}=10.3 \mathrm{mM}$. Below that concentration,

a)

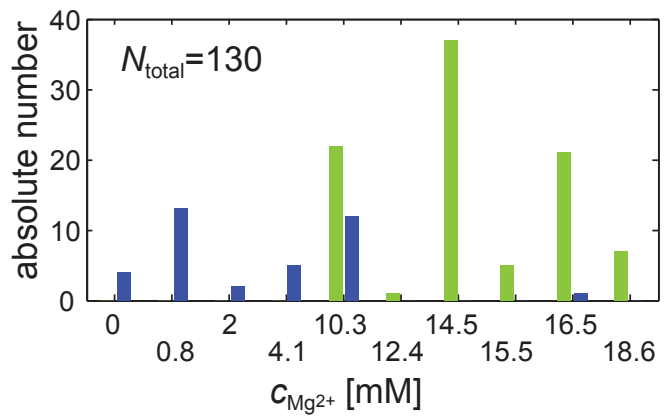

c)

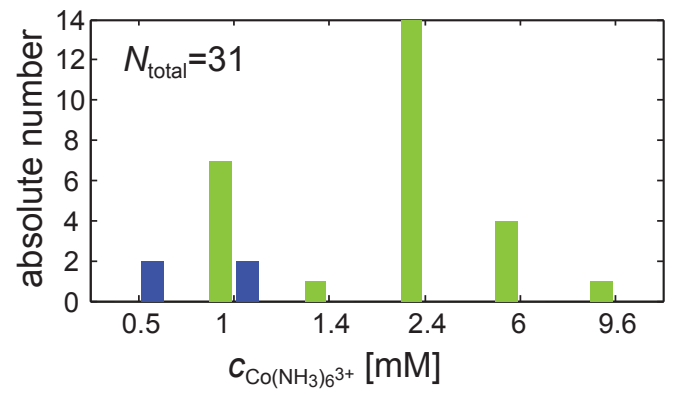

e)

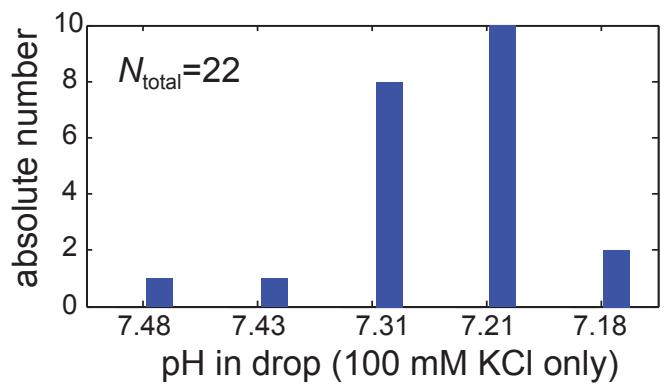

b)

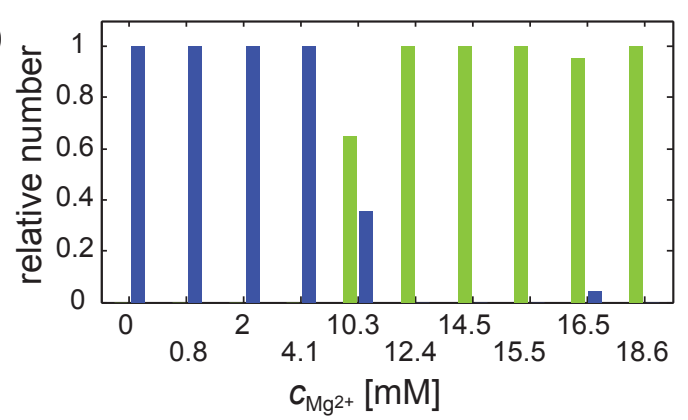

d)

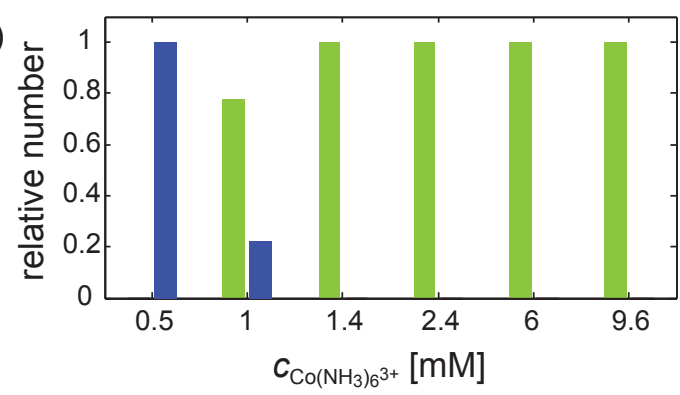

color code

filament aggregation no filament aggregation

Figure 7.4: Qualitative drop content analysis in the device T. The qualitative analysis shows that filament aggregation starts at $c_{\mathrm{Mg}^{2+}} \approx 10 \mathrm{mM}(\mathrm{a} / \mathrm{b})$ and in the range of $c_{\mathrm{Co}\left(\mathrm{NH}_{3}\right)_{6}^{3+}} \approx 0.5-2.4 \mathrm{mM}(\mathrm{c} / \mathrm{d})$. Variations in $\mathrm{pH}(7.2$ to 7.5$)$ do not influence the free fluctuation of vimentin filaments as we observe no aggregation in all cases (e). $N_{\text {total }}$ gives the total number of drops that are considered in a), c) or e), respectively. 
no aggregation is observed and above this concentration in almost all cases the filaments aggregate. At $10.3 \mathrm{mM}$, the outcome of the experiments seems to be heterogeneous. As we identify a threshold of aggregation, this heterogeneity is explained in the light of small flow rate dosing inaccuracies or effects of magnesium ion inaccuracies by drop shrinkage (Sec. 5.2). This inaccuracies explain that the actual magnesium chloride concentrations can show a small variation and therefore clarify the observed heterogeneity at $10.3 \mathrm{mM}$. The aspect of concentration accuracies is further discussed in Sec. 9.1.3.

In addition to our study with magnesium ions, we also test the aggregation behavior of vimentin in the presence of hexammine cobalt chloride (Fig. 7.4c/d). In these experiments, the onset concentration of aggregation is notably lower compared to magnesium ions. In all cases, we observe aggregation for $c_{\mathrm{Co}\left(\mathrm{NH}_{3}\right)_{6}^{3+}} \geq$ $2.4 \mathrm{mM}$ and this result is based on the analysis of 19 drops. For $c_{\mathrm{Co}\left(\mathrm{NH}_{3}\right)_{6}^{3+}}<$ $2.4 \mathrm{mM}$, the data show a heterogeneity that is interpreted as a threshold like in the case of magnesium chloride. As we observe no aggregation for $c_{\mathrm{Co}\left(\mathrm{NH}_{3}\right)_{6}^{3+}} \geq$ $0.5 \mathrm{mM}$, the threshold for $c_{\mathrm{Co}\left(\mathrm{NH}_{3}\right)_{6}^{3+}}$ is therefore in the range of $0.5 \mathrm{mM}-2.4 \mathrm{mM}$. The lower bound of $0.5 \mathrm{mM}$ might need further confirmation by more measurements. Nevertheless, these results are convincing in terms of two aspects. First,

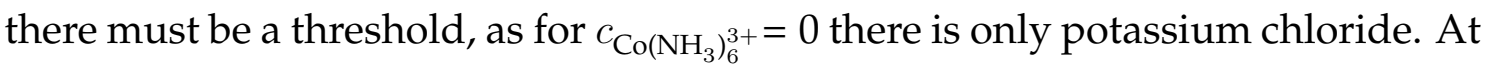
these filament assembly buffer conditions no aggregation is observed. Second, the threshold is below $2.4 \mathrm{mM}$ as all the data at these concentrations indicate filament aggregation.

In additional experiments with multivalent ions, drops without any multivalent salt content were produced. Instead, the component aq 1 (Fig. 4.4) contained $100 \mathrm{mM} \mathrm{KCl}$ at a $\mathrm{pH}$ other than 7.5. In this way, we tuned the $\mathrm{pH}$ in the drop. The experiments clearly indicate that no aggregation occurs in the $\mathrm{pH}$ range of 7.18 to 7.5 (Fig. 7.4e). This is a further check, as the observed filament aggregation is not influenced by small inaccuracies in $\mathrm{pH}$ adjustments of the chemicals. Additionally, this also verifies that at our filament assembly conditions, potassium chloride only does not lead to vimentin aggregation (no multivalent ions present). 


\subsubsection{Details on aggregation over time}

In this section, we investigate how filament aggregation evolves over time. For this purpose, we study in detail drops with a prolonged residence time in the drop trap (dwell time $>60 \mathrm{~s}$ ). In Fig. 7.5, five examples of these time-lapse experiments are given. On a qualitative level, the observed network morphologies are grouped into three classes. In the first case, the filaments do not show an aggregation or attraction mechanism. Instead, they fluctuate freely during the whole observational period. These morphologies are found for magnesium concentrations in the range of $c_{\mathrm{Mg}^{2+}}=0.8-4.1 \mathrm{mM}$ (see example in Fig. 7.5a). We chose a magnesium concentration of $c_{\mathrm{Mg}^{2+}}=14.5 \mathrm{mM}$ to investigate the aggregation mechanism (see Fig. 7.5b-e). This magnesium concentration is well above the threshold concentration for the onset of aggregation (Sec. 7.1 and 7.2.1).

For these cases, we sort the networks into two groups. The first group is given by networks which spread over a larger region in the drop (Fig. 7.5b,c). These networks are like a scaffold as more and more free filaments in the drop attach to this scaffold firmly over time. Attached to this scaffold, their fluctuation is largely reduced. For the second group, the filaments also attract to each other. But instead of a scaffold-like widespread aggregate, the final state is more a point-like aggregate that gets more and more compact over time. Gradually, the vimentin filaments are firmly integrated into these aggregates as well, while the network as a whole is compacted in parallel. In both the latter cases, an attraction principle is clearly recognizable.

The reason for the two different network formation behaviors (scaffold-like vs. point-like aggregation) is not well understood as random processes seem to be involved. However, the fundamental principle of network formation can be illustrated by the idea of an 'energy landscape': Locally, filaments start to aggregate randomly. Over time a network emerges. The first option is that the network evolves into a scaffold-like structure. This construct is stabilized against further compaction at some point, as the network is 'trapped' in a local energy minimum. It has little potential for further compaction as a high energetic barrier separates its current energetic state from a lower energy minimum. The second option is that there is no stabilization over the micrometer range. Then the network is not stable and compacts more and more. This way the network reaches an overall very low energetic state. In the end, this network becomes point-like. In any case, the mechanism is observed as a local interplay of filament attraction, net- 
work compaction and the stabilization as a result of the attraction.

In Fig. 7.6a-c, we give a detailed view on these principles of aggregation. A

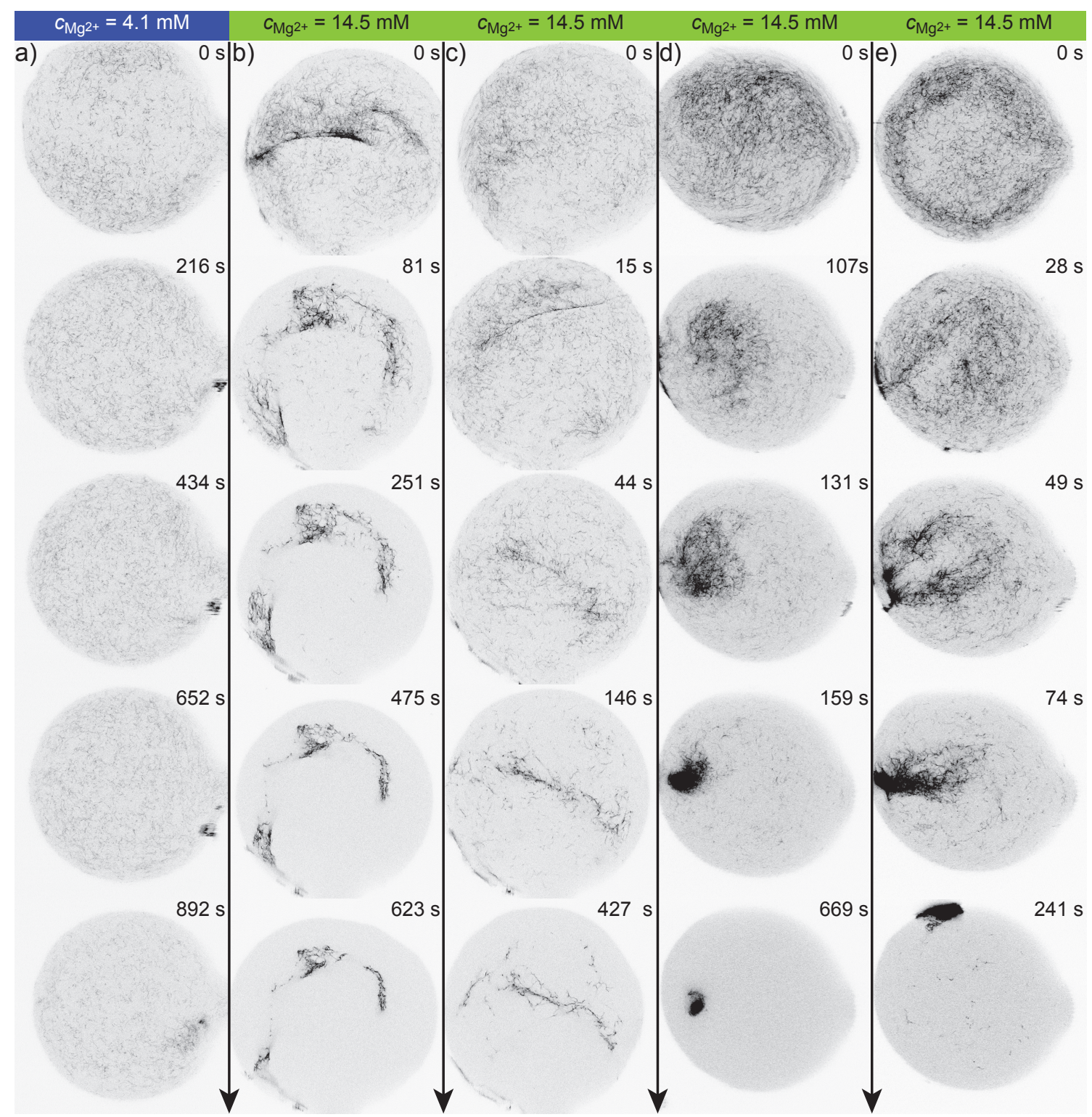

Figure 7.5: Time-resolved vimentin aggregation observed in trapped drops. For different magnesium chloride concentrations (a: $4.1 \mathrm{mM}$, b-e:14.5 mM), networks of filaments (inverted grayscale fluorescence) are shown at time points relevant for each timelapse series. The network morphologies are classified into free filaments (a), scaffold-like or elongated networks of filaments $(b, c)$ and point-like aggregates $(d, e)$. The side length of the images is $106 \mu \mathrm{m}$. The grayscales for the different time series studies were slightly adjusted for displaying purpose. This correction procedure was the same within a time series. (Partly adapted from reference [35] with permission from The Royal Society of Chemistry) 
a)
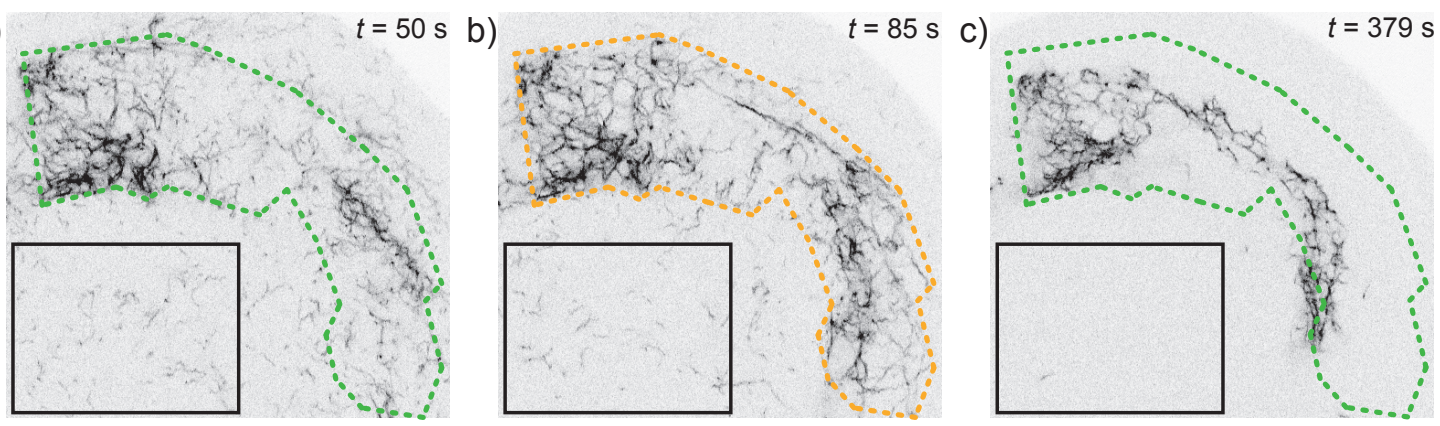

d)
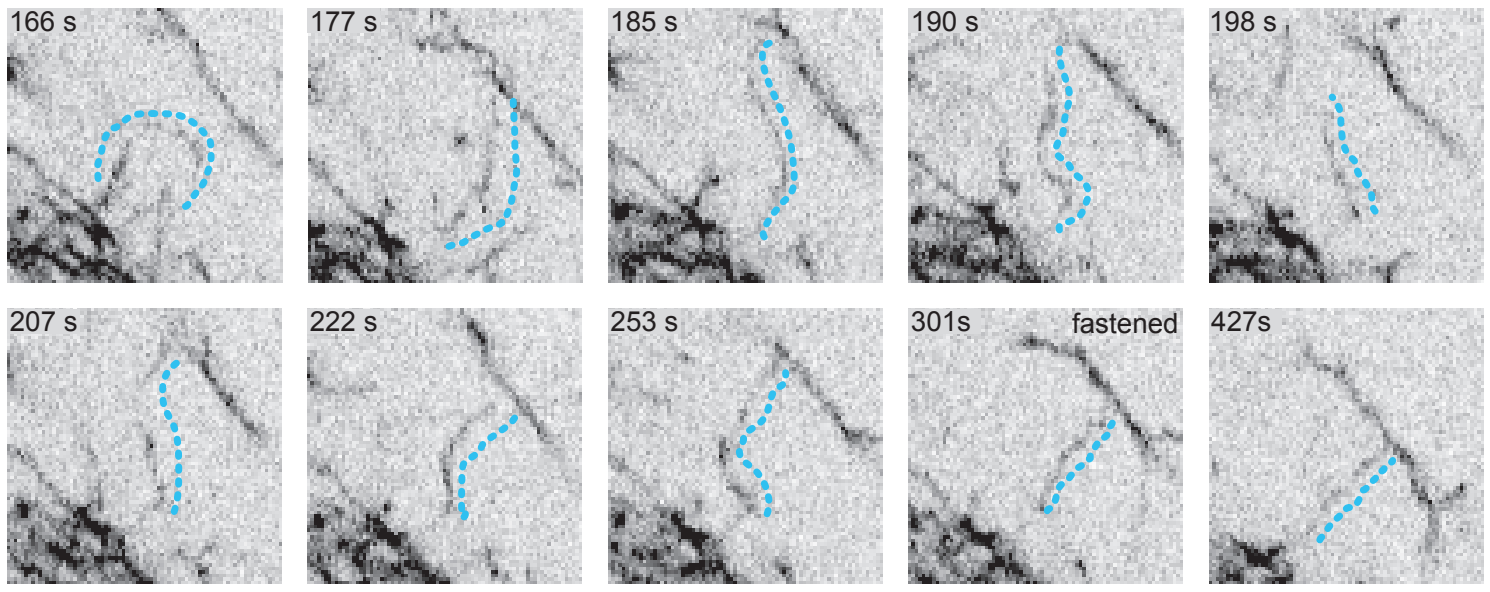

Figure 7.6: Principles of network formation. a-c) Details of the network shown in Fig. 7.5b. The outline of the network already connected in b (orange) is also plotted in a) and c). The comparison of this outline to the networks in a) and c) illustrates the compaction mechanism. From a)-c) the number of free filaments (black boxes) decreases as the filaments are integrated into the main network (see also Fig. 7.5b-e). In d) the firm attachment of filaments to other filaments is illustrated over time (part of Fig. 7.5c). The dotted line illustrates the shifted contour line of the filament as it first fluctuates markedly (166 s to $301 \mathrm{~s})$. After $301 \mathrm{~s}$, its fluctuations are notably reduced due to the firm integration into the local network structure. At the same time, the whole network stabilizes. Therefore, it becomes more rigid and self-stabilizes its morphology. This example shows the general mechanism of reduction of the filament fluctuation, as it is integrated into the network.

typical appearance are filaments or filament bundles that are 'half-bound' at the periphery of the main aggregate: One part of the filament is attached to the network whereas the other is freely fluctuating. The free filament part fluctuates until it comes close enough to another filament to become part of the network aggregate (Fig. 7.6d).

The details of the network structure are analyzed using the method of image binarization and convex hull detection as described in Sec. 6.2. We take the relative convex hulls that covers at least $\mathrm{CI}=90 \%$ of the the network intensity (Fig. 7.7). At low magnesium ion concentration, no aggregation of vimentin filaments to 
a)

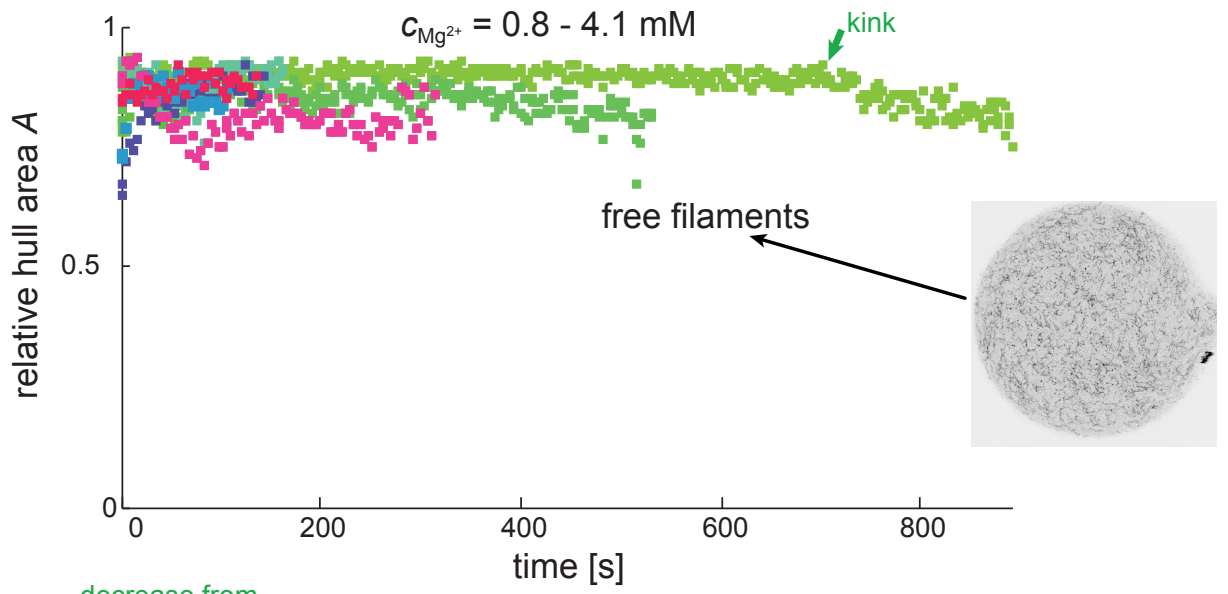

b)

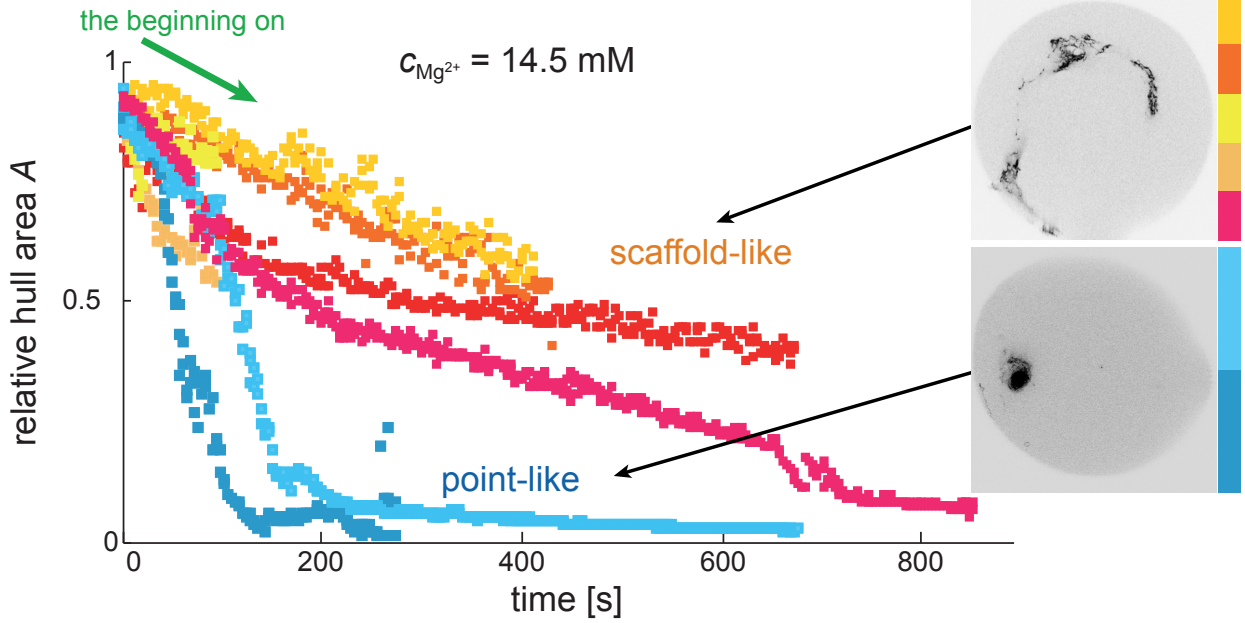

Figure 7.7: Relative convex hulls of vimentin networks during aggregation. a) For low magnesium ion concentrations $\left(c_{\mathrm{Mg}^{2+}}=0.8-4.1 \mathrm{mM}\right)$, the relative hull area $A$ is close to 0.9 for all cases. This indicates that the filaments are distributed equally over the whole drop (Fig. 6.9). b) For $c_{\mathrm{Mg}^{2+}}=14.5 \mathrm{mM}$, the aggregation of the filaments to networks of filaments start from the very first moment of observation, indicated by decreasing slopes. The point-like networks aggregate fast into compact structures. In contrast, scaffold-like networks show a shallower slope and their compaction takes longer. Each color represents the data points for the network in one drop. (Adapted from reference [35] with permission from The Royal Society of Chemistry)

networks is expected. Accordingly, the relative convex hulls are largely constant (Fig. 7.7a). Small deviations from a more or less constant value are explainable: For instance, the longest curve, which corresponds to the network in Fig. 7.5a, has a 'kink' in between 700 and $800 \mathrm{~s}$. This is due to the severe bleaching of the fluorescence label. However, the analysis method of the convex hull proves robust against this influence as only little decrease is observed.

For a magnesium concentration favoring network formation $\left(c_{\mathrm{Mg}^{2+}}=14.5 \mathrm{mM}\right)$, 
a)

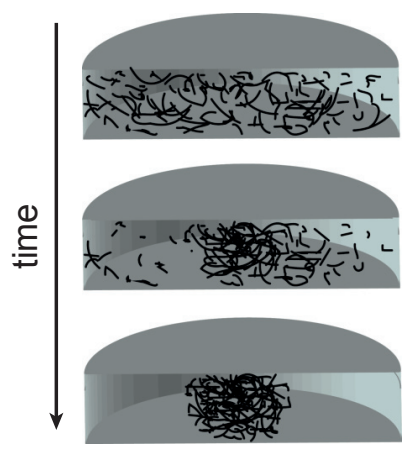

b)

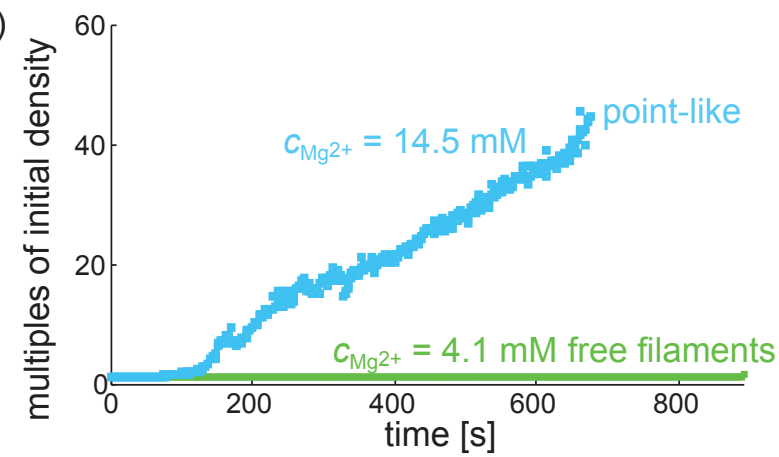

Figure 7.8: Maximum network compaction. a) The volume occupied by the filaments is reduced during the time course of vimentin aggregation in the oblate drops. b) For the case of maximum compaction, we observe that the mass density increases by the 45 -fold compared to the initial mass density in the drop. This demonstrates the high ability of multivalent ions to mediate strong filament attraction. (Adapted from reference [35] with permission from The Royal Society of Chemistry)

we identify once more the two classes of network morphologies based on their hull area: Point-like aggregates decrease rapidly in size whereas scaffold-like networks show less overall compaction (see Fig. 7.7b). In all cases, the aggregation curves decrease directly from the first seconds of observation. Therefore, the influence of the multivalent ions is directly mediating attraction between the filaments. It is important to investigate how much compaction can in principle be induced by the presence of multivalent ions. Therefore, we investigate the maximum compaction we found in our experiments (Fig. 7.8). In this example, the volume of the point-like network is estimated based on the geometry of a cylinder spreading over the entire height of the oblate drops $(18 \mu \mathrm{m}$ height, $\approx 100 \mu \mathrm{m}$ diameter). The base area of the cylinder is approximated by the convex hull area. This estimation yields a maximum compaction of the 45 -fold compared to the initial mass density of the filaments in the drop. In the highest compaction state, the point-like aggregate has a diameter of about $7-11 \mu \mathrm{m}$. For further compaction, the persistence length of vimentin (2 $\mu \mathrm{m}$ [93]) might act as a barrier.

In addition to the time-lapse studies of vimentin in the presence of the divalent magnesium ions, we also tested the influence of the trivalent hexammine cobalt ions. Examples for the behavior of the filaments over time are given together

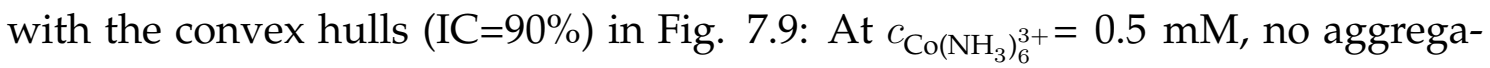
tion of the filaments is observed (a). In contrast, an example of a scaffold-like network is given (b). For these two examples, the filaments show no deviation from the behavior of vimentin in the presence of magnesium: Where the fila- 


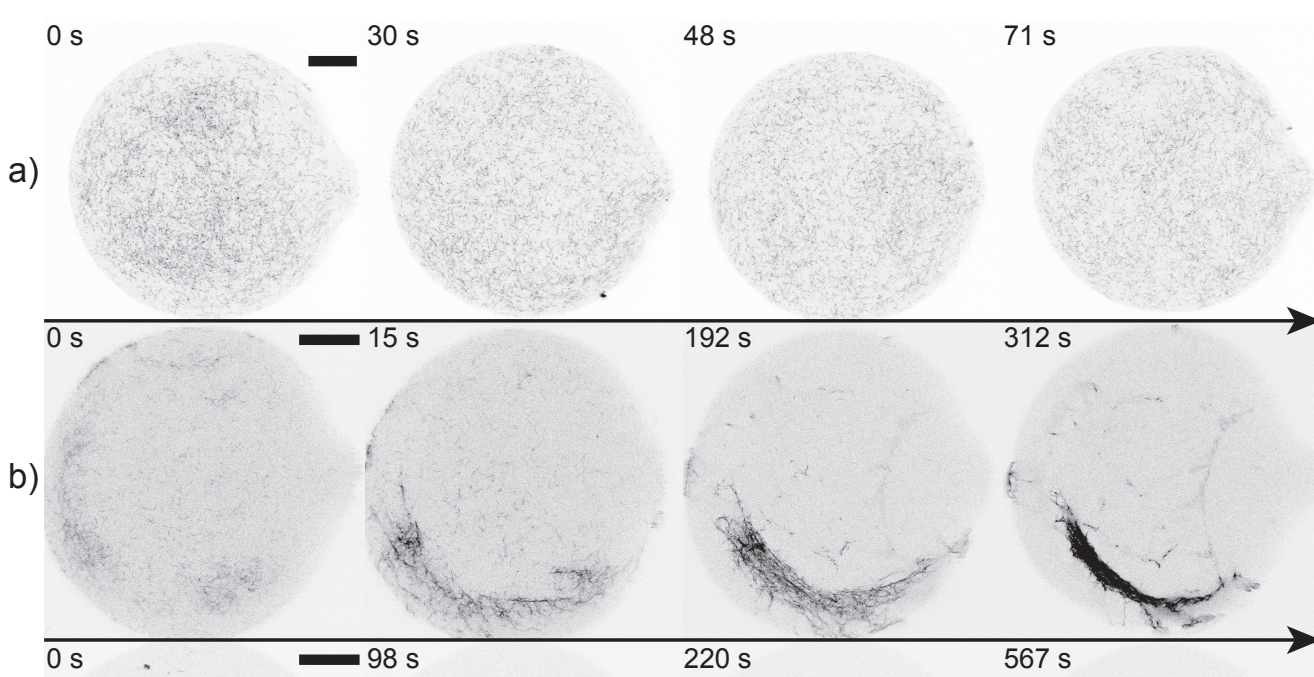

c)

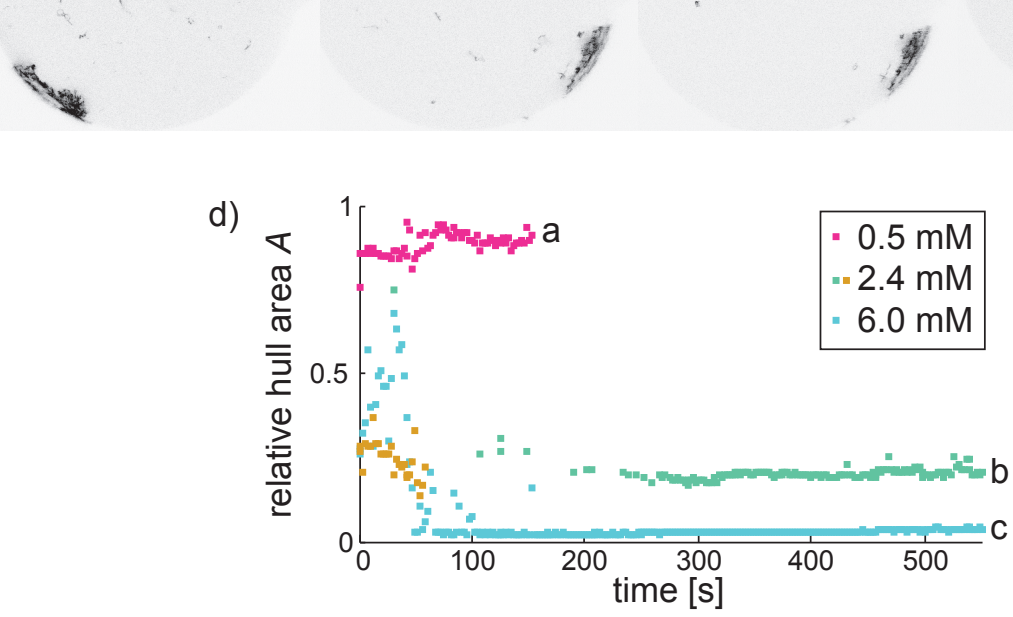

Figure 7.9: Interaction of vimentin filaments with hexammine cobalt chloride

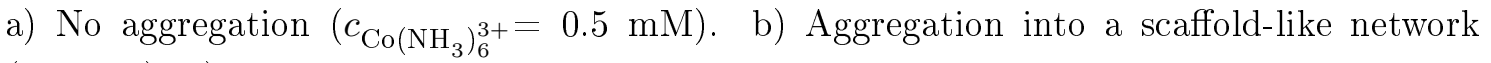
$(2.4 \mathrm{mM})$. c) Point-like aggregation: The filaments arrive highly aggregated in the drops. The corresponding convex hulls are given in d). The scale bars are $20 \mu \mathrm{m}$. (Adapted from reference [35] with permission from The Royal Society of Chemistry)

ments do not aggregate, they just show thermal fluctuations and therefore the convex hull is also constant around $A=0.9$. For the example in $\mathrm{b}$ ), the network is also compacted over time and free filaments are integrated into the main aggregate. Only the time scale of aggregation (mainly within the first $\approx 100 \mathrm{~s}$ ) is faster compared to any experiment with magnesium (Fig. 7.7). Qualitatively spoken, we observe the same aggregation principles at this concentration as in the presence of $c_{\mathrm{Mg}^{2+}}=14.5 \mathrm{mM}$. For the example $\mathrm{c}(6 \mathrm{mM})$, the vimentin aggregate is 
already present when the drop arrives in the drop trap. It is not clear whether this observation is an experimental artifact or whether it indicates a new aspect of aggregation. It may be that the drop was delayed during its passage through the device before imaging. However, this is rather unlikely as we never observed any drops that were 'stuck' in the microfluidic pathways. Nevertheless, it is consistent that aggregation occurs at $6 \mathrm{mM}$. Additionally, the few filaments that are initially in the drop vanish over time and are therefore also integrated into larger filament clusters.

\subsection{Time-scales in the aggregation process}

Using the method of the convex hull analysis, we investigate the morphologies of the aggregating vimentin filaments over time. One characteristic feature is that the curves decrease to small hull values, i.e. small aggregates, within a time span of $10 \mathrm{~min}$ (Fig. 7.7). Here, we investigate whether the observed time scales for aggregation can be explained and to which extent. For this investigation, the

a)

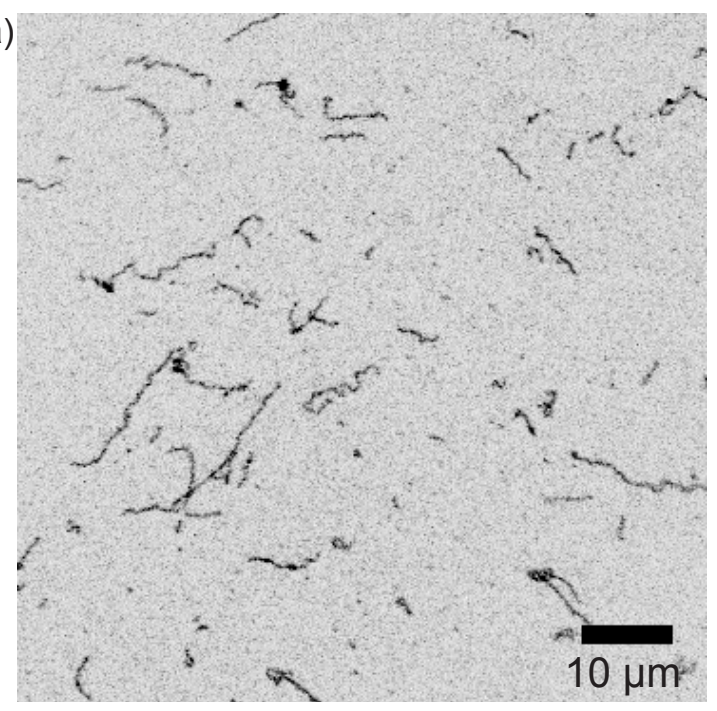

b)

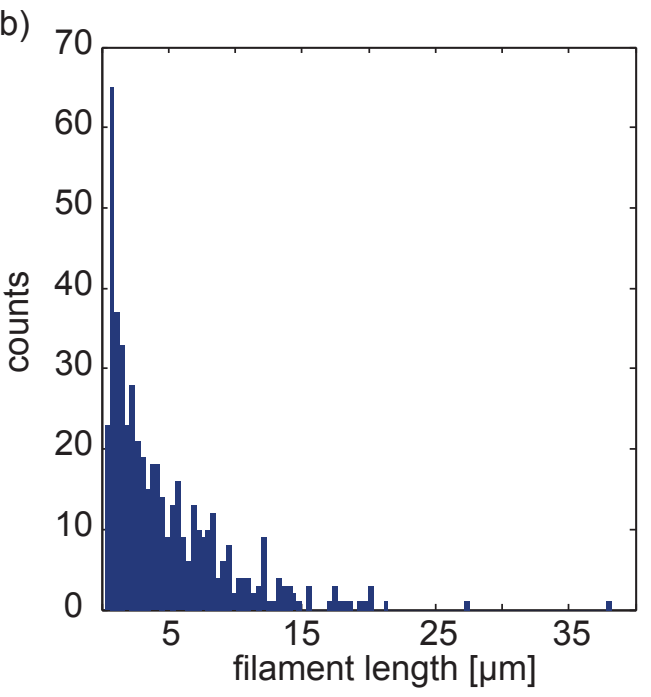

Figure 7.10: Filament length distribution. a) Selected region of vimentin on a cover glass. b) The measurements of the contour length for 500 filaments are shown in a histogram. The mean contour length is $5 \mu \mathrm{m}$. For this measurement, filaments that are assembled following assembly protocol B are diluted 160-fold with assembly buffer (Sec. 3.1.3). With the help of this dilution, the individual filaments are clearly identified. The analysis represents those filaments that are resolved using the confocal microscope. However, the filament length should exceed $200 \mathrm{~nm}$ rapidly during the filament assembly process as filament assembly on this length scales is expected within one minute. [51] 
length scales over which attraction force is mediated are important. The order of magnitude for this force is accessible. The investigation of the mixing process in the drops shows that the multivalent ions are everywhere in the drop when the drops arrive in the traps (Sec. 5.1). Consequently, the ions around each filament interact directly with the filament. The length of Debye charge screening and the Gouy-Chapman length are on the order of $1 \mathrm{~nm}$ or below (Sec. 2.2 and 8, Tab. 8.2 and 8.4). This circumstance justifies the assumption that an attraction between two filaments in the presence of multivalent ions can only be mediated when the minimal distance between two filaments is below a threshold distance on the order of $1 \mathrm{~nm}$. To illustrate this, we point out that the attraction only takes place for those filaments which are in the same pixel of an image in our experiments. This implies that two filaments have to approach closely until the attractive forces are relevant. There are two mechanisms by which the filaments are brought together. First, the filaments approach by diffusion. Second, flow of the surrounding buffer brings them closer together. In contrast to flow, diffusion must be present.

To estimate the effect of diffusion, the distribution of the contour length of filaments is investigated in detail for the experiments in the device T (Fig. 7.10). The distribution of filament lengths reaches from $200 \mathrm{~nm}$ to about $20 \mu \mathrm{m}$. Since we are only interested in an estimation of the diffusion effect, we consider all protein filaments as $5 \mu \mathrm{m}$ long filaments (average filament length). Initially, these filaments are distributed homogeneously in the whole drop to a good extent (Sec. 5.1). For an initial configuration, the situation is similar to the case of filament distribution in drops where there is no aggregation (Fig. 7.5a). From these images we determine a typical length scale of 1-2 $\mu \mathrm{m}$ as a minimal distance between two neighboring filaments (Fig. 7.11a). For this estimation, we measure the minimal distance of randomly chosen neighboring filaments. This quantity gives an estimate of the space in between two neighboring filaments and is therefore considered as the diffusion length that the filaments have to overcome before attraction mediated by multivalent ions takes place. The diffusion time for a $5 \mu \mathrm{m}$ filament is less than one second on this length scale. Additionally, rotational diffusion of filaments might be necessary so that two filaments can attach to each other (Fig. 7.11b). In the upper limit of a rectangular angle between the two filaments, the rotational time is $7 \mathrm{~s}$. Overall it takes less than $10 \mathrm{~s}$ for two $5 \mu \mathrm{m}$ filaments to connect to each other. After this first contact, an aggregation 'cascade' is expected: Two filaments associate along their contour length ('zipping') 
a) inter-filament distance

$$
\text { , }
$$

b) rotational diffusion

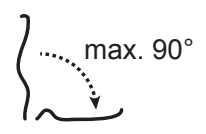

\section{c) aggregation prior to trapping}
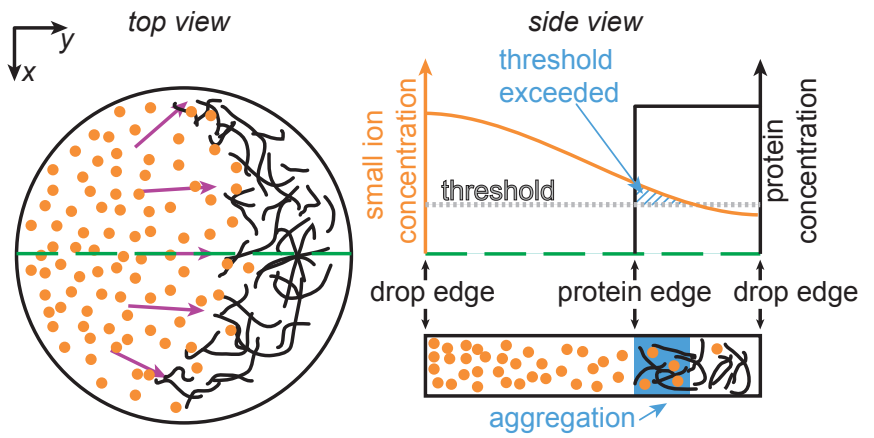

d)

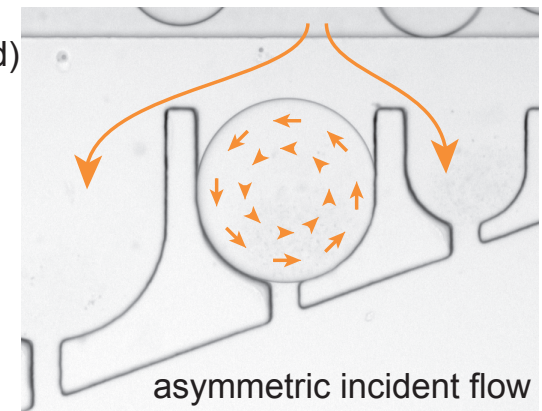

e) flowing domains

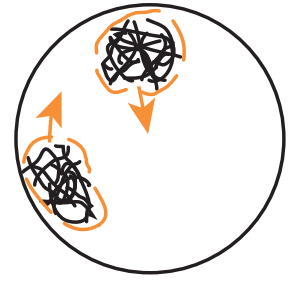

f) local attraction

g) compaction

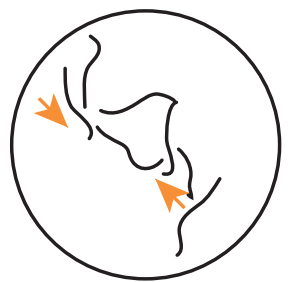

Figure 7.11: Diffusion and flow in the drops. a) Initial inter-filament distance. b) Rotational diffusion. c) Directly after drop production, the mismatch in the diffusion constant of small ions and the filaments could lead to the concentration distribution as sketched in the image. When the ions locally exceed the threshold concentration, networks of filaments can already form. d) An asymmetric shear flow around the trapped drop evokes a circular flow in the drop. The internal flow patterns can also be more complex leading to the flow of domains (e). The movement of the filament and their network is also possible via compaction $(f, g)$.

and a cluster of two filaments interact with filaments or other clusters. The exact morphology of these clusters is unknown. However, the quality of cluster diffusion is known. The diffusion constant of a cluster is most likely smaller compared to the filament. Therefore, further network connection slows down over time. In summary, the analysis of the diffusion time that is needed for the first filamentfilament connection provides an explanation for the onset of aggregation right from the beginning of the drop trapping. This is represented by the decrease of the convex hull directly after drop trapping (Fig. 7.7b). The deceleration of the aggregation leads to shallower slopes of the convex hull curves over time (Fig. 7.7b).

Besides the diffusion process, other phenomena are important for the time scales of the aggregation process in the trapped drops. When the three aqueous com- 


\section{a) circular flow}

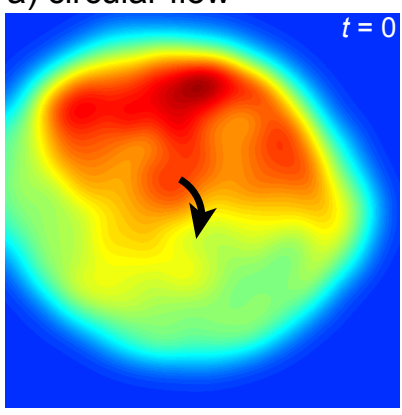

b) flow domains

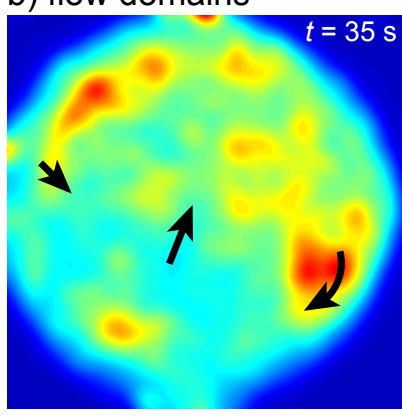

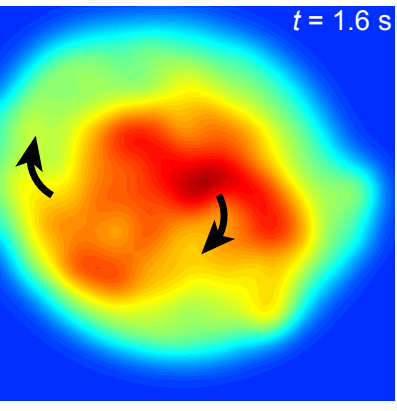

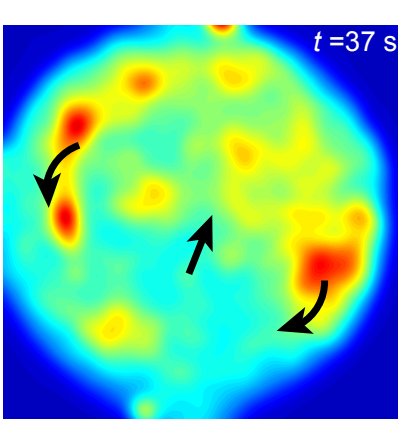

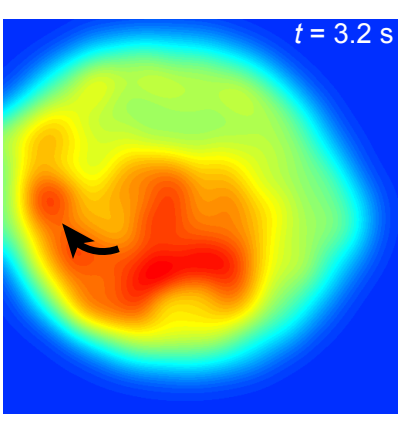
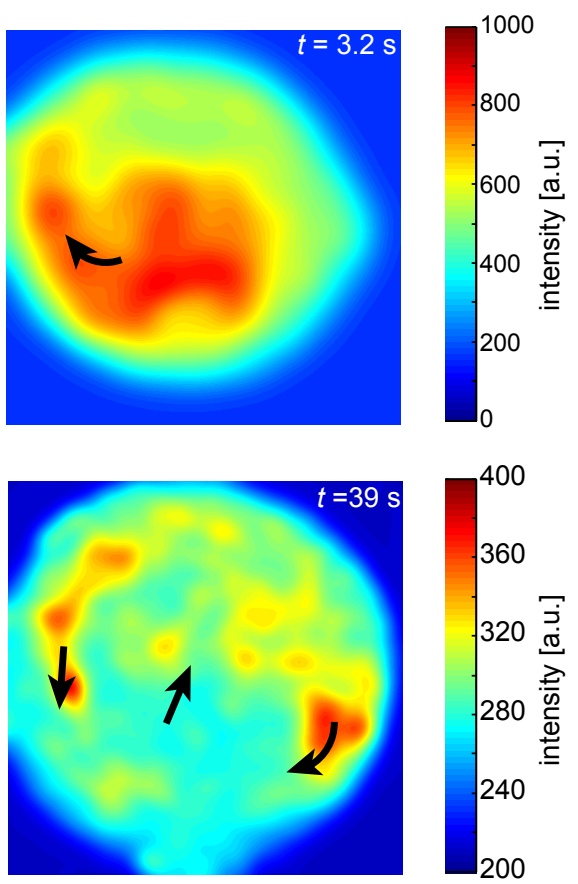

Figure 7.12: Illustration of flow in the drop interior. The raw data in these images are highly smoothed (Gaussian filter) so that domains of clusters of filaments are visible. The intensities [a.u.] are then color coded. a) Example of a circular flow. (Images belong to the image series as shown in Fig. 7.5). d) In this example, there is a more complex flow field leading to the movement of the domain in a non-circular way. The movement of the domains is indicated by the arrows.

ponents are encapsulated into the drops, they also have a side-by-side arrangement in the drops, until they are mixed in the serpentine or straight channel (Sec. 5.1). This is shown in Fig. 5.6b. The mismatch of the diffusion constant of the small multivalent ions $\left(D_{\mathrm{mi}} \approx 10^{-9} \mathrm{~ms}^{-2}\right)$ and the filaments $\left(D_{\text {vimentin }} \approx\right.$ $10^{-13}-10^{-12} \mathrm{~ms}^{-2}$ ) might also be important (Sec. 5.1). As the small ions are much faster than the filaments, they might distribute in the drop so fast that locally the ion concentration exceeds a threshold concentration in the region where the filaments are located (Fig. 7.11c). Initially, the protein concentration in this regions is three times as high compared to a homogeneous filament distribution in the whole drop. Consequently, any inter-filament distances are three times smaller, and any diffusion times take only $1 / 9$ of the time that is found in the approximations made above (Eq. 2.28). Therefore, the times for translation diffusion are often below $100 \mathrm{~ms}$. Due to this estimation, it is reasonable to assume that the first filament-to-filament connections are formed from the very first moment of drop production. The aggregation morphology, as depicted in Fig. 7.11c, 
is also in agreement with the scaffold-like networks as shown in Fig. 7.5b/c. The central backbone of the network has the dimension and the bending of the drop outline. The shape might be influenced by this factor.

When the drops are situated in the traps, we observe flows in the drop interior. These flows are visible, as the fluorescent filaments also act as tracer particles, indicating flow velocity that is clearly not due to filament-filament attraction. We expect the incident flow to the drop as the reason for this mechanism. For instance, an asymmetric flow leads to a circular internal flow (Fig. 7.11d and 7.12a). Besides the circular flows, we also observe more complex flow fields, in which entire domains flow in different directions (Fig. 7.11e and 7.12b). These flows are more pronounced in some drops than in others. They are independent of the formation of vimentin networks. Consequently, this effect is independent of the protein. Nevertheless, these internal flows influence the network formation, due to the material transport that is associated with them. As already shown in Fig. 7.6, free filaments are transported by these flows towards the main aggregate in the drop. There is a chance that these filaments stick to the main aggregate. Consequently, these flows accelerate the time needed for aggregation. By tracking single filaments the flow velocity in different drops is estimated to be about $5 \mu \mathrm{m} \mathrm{s}^{-1}$. The individual filaments do not follow a regular flow pattern over time. Therefore, this flow speed shows large deviations. Nevertheless, it determines the order of magnitude of time of this process. Thus, a single filament can pass the whole drop diameter $(100 \mu \mathrm{m})$ in $20 \mathrm{~s}$. The average time that passes until an unbound filament is attached to another filament increases over time as the network aggregates. This decelerated aggregation can be attributed to the fact that there are fewer binding partner per volume as the aggregation process proceeds.

In addition to diffusion and flow phenomena, the third influence on the compaction process is the ability of network interiors to attract each other locally. The overall attraction leads to the compaction of the entire network (Fig. 7.12f/g). An example of this compaction is given in Fig. 7.6a-c. This process is interesting, since a method of 'self-stabilization' is expected: The more compact the network gets, the stiffer it becomes. As a consequence, the shrinkage rate of the network volume decreases. In contrast to all other time scales, this process takes much longer. We estimate that the compaction process proceeds over a time span of about $100 \mathrm{~s}$ (see examples in Fig. 7.5b-e and 7.6a-c). 


\begin{tabular}{ll}
\hline process & time scale $[\mathrm{s}]$ \\
\hline interaction of ions and polyelectrolyte filaments & $\ll 1$ (immediately) \\
aggregation prior to trapping (within & $0.1-5$ \\
$1-5$ s after drop production) & \\
filament diffusion to next filament & $1-10$ \\
cluster diffusion & $>10-\gg 10$ \\
drop internal flow $(\approx 5 \mu \mathrm{m} / \mathrm{s})$ & $>20$ \\
network compaction & $>100$ \\
\hline
\end{tabular}

Table 7.1: Order of magnitude of the time scales of different processes involved in the network formation.

Based on the consideration discussed above, we compare the different time scales of the different processes in Tab. 7.1. In conclusion, the time scales of aggregation are the result of several processes which in sum determine the value of the overall time scale. The early onset of filament aggregation is a result of the diffusion properties of small metal ions and protein filaments. Importantly, the observed aggregation is not due to flow in the drops leading to 'entangled' filament networks. This is excluded because there is also internal flow in the drops in the presence of low magnesium concentrations (example in Fig. 7.5a). However, it is not possible to identify a single process that determines the time scale. Nonetheless, at an early stage, diffusion and drop internal flow are important. When a network is already established later on, the morphology of the network gets more and more independent of these influences. Then, the stiffness of the network is the most important factor. The estimates performed above depend on the concentration of the filaments. When more filaments are present, the paths to travel by diffusion are smaller and more binding partners are available for the filaments. However, the network stiffness might also change drastically. When the meshes of the network are smaller, the stiffness might also be higher.

\subsection{Studies without drops}

\subsubsection{Vimentin studied on cover glasses}

A complementary approach to study the interaction of vimentin and salt is to mix protein filament solution and a salt buffer in a test tube and spread the mixture 
a)

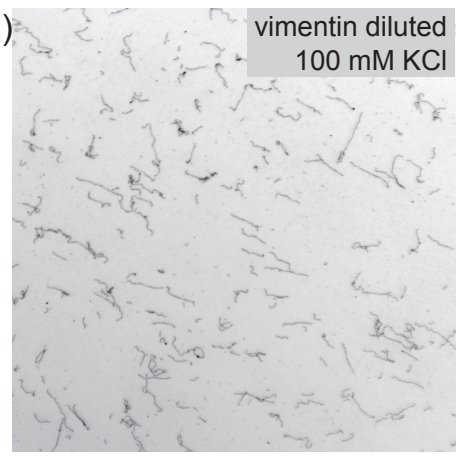

b)

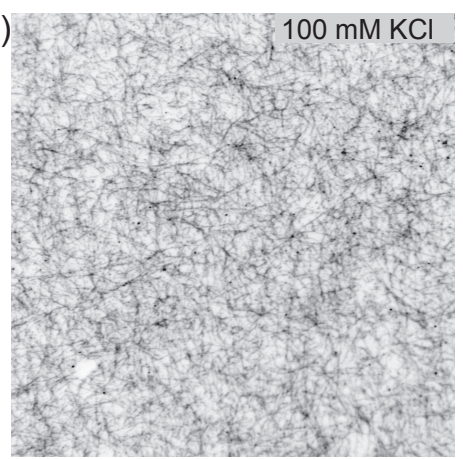

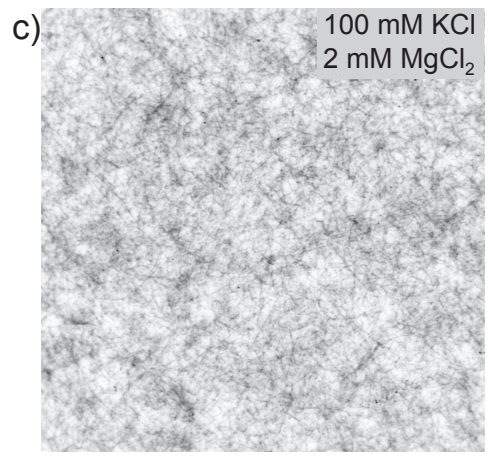

d)

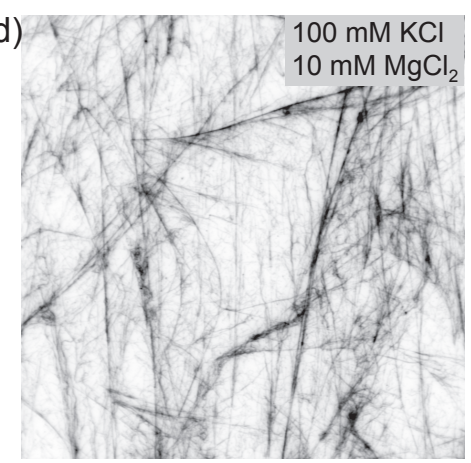

e)

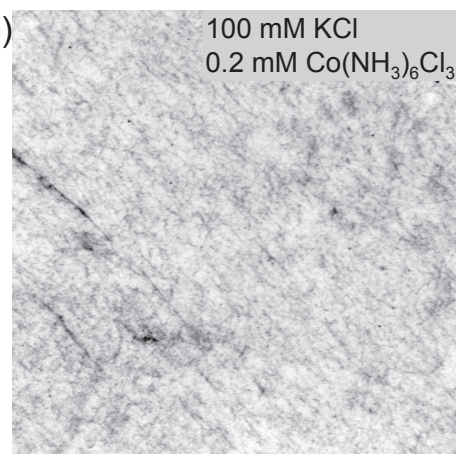

f)

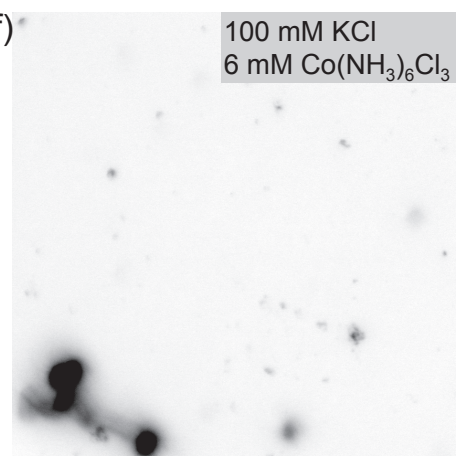

Figure 7.13: Vimentin studied on a cover glass. a) Diluted vimentin at assembly buffer conditions. b) The undiluted filaments spread homogeneously over the cover glass. The same homogeneous spreading is observed for low magnesium chloride (c) and low hexammine cobalt (e) concentrations. d) For higher magnesium concentrations, long filament bundles and network formation are observed. f) At $6 \mathrm{mM}$ hexammine cobalt, the result are fragments of vimentin aggregates. The side length of all images is $100 \mu \mathrm{m}$.

onto a cover glass (Fig. 7.13). For these experiments, the chemical composition is the same as in the experiments with drops studied in the device $\mathrm{T}$ (Sec. 7.2). Only for one experiment, illustrated in Fig. 7.13a, the concentration of vimentin filaments is diluted 160 times compared to the concentration in the drops. This is another way of checking that the filaments form no networks at assembly buffer conditions. When the protein solution is poured onto the cover glass, the filaments attach firmly and show absolutely no thermal fluctuations. To decrease the influence of fluorescence from off-focus planes, the cover glasses in Fig. 7.13b-f are flushed for a short moment with buffer (filament assembly conditions), then epifluorescence images are taken. There is no evidence that the filaments attached to the cover glass are removed through the washing step. Consequently, there is a strong interaction between vimentin filaments and glass. The structures for the conditions as given in b), c) and e) all show no filament aggregation. (b: filament assembly conditions, $\mathrm{c}: c_{\mathrm{Mg}^{2+}}=2 \mathrm{mM}$ and e: $\left.c_{\mathrm{Co}\left(\mathrm{NH}_{3}\right)_{6}^{3+}}=0.2 \mathrm{mM}\right)$. In contrast, 
at $10 \mathrm{mM}$ magnesium ion concentration, distinct filament bundling and network formation is observed (d). For $c_{\mathrm{Co}\left(\mathrm{NH}_{3}\right)_{6}^{3+}}=6 \mathrm{mM}$, the outcome is challenging to interpret (f): Some filament knots are visible, but overall no defined structures are found. At least it is apparent that the trivalent hexammine cobalt ions have a strong influence on the filaments at the given concentration.

The results obtained on the cover glasses are in agreement with the experiments performed in drops (Sec. 7.1 and 7.2). The onset of aggregation corresponds to the threshold of ion concentration that were also observed in the drops. Concerning the morphology of the network structures, a difference is observed between the experiments on the cover glasses and the drop experiments: The network at $c_{\mathrm{Mg}^{2+}}=10 \mathrm{mM}$ (Fig. 7.13d) has wider meshes and longer fibers in comparison to networks found in the drops (Fig 7.5). This aspect is further discussed in Sec. 9.2.

\subsubsection{Networks with vimentin mutants}

The reason for vimentin filament aggregation in the presence of multivalent ions are of fundamental importance. In the literature, the tail region of IFs is believed to play a key role in the process of inter-filament attraction. [16, 25, 52, 63, 76, 77] One hypothesis is that the tail region is essential for the formation of crosslinks in networks. To investigate the role of the tail, we perform experiments with the vimentin mutants $\Delta \mathrm{C} 411$ and $\Delta C 455 .^{1}$ In these experiments, different vimentin mutants are assembled separately following assembly protocol B (Sec. 3.1.3). The vimentin mutant that is closest to the wild-type protein is labeled with Alexa 488 dye and both $\Delta C 411$ and $\Delta C 455$ are labeled with Atto647N. The static measurements of vimentin networks on a cover glass are sufficient to investigate whether the (partly) tailless vimentin mutants are built in the networks of vimentin. We mix vimentin filaments with one of the two mutants $\Delta C 411$ or $\Delta C 455$, add magnesium chloride dissolved in assembly buffer and image the resulting network on a cover glass (see Fig. 7.14). These images show the inverted fluorescence in grayscales for the channel of vimentin and the mutant. The filament length of the mutant is different compared to vimentin, which might be due to different protein concentrations (Sec. 3.1.3). The channels are merged to illustrate the colocalization between vimentin and the mutant. In part, the local positions and orientations of both filaments directly match (examples: arrows in Fig. 7.14a/c).

\footnotetext{
${ }^{1}$ Details on the mutants are documented in Sec. 3.1.1.
} 


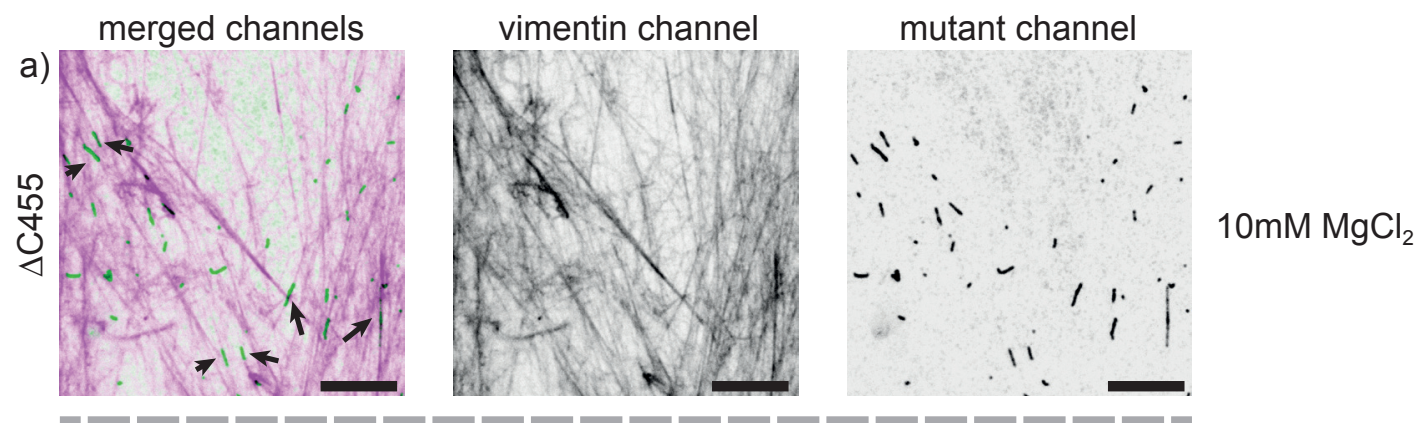

b)

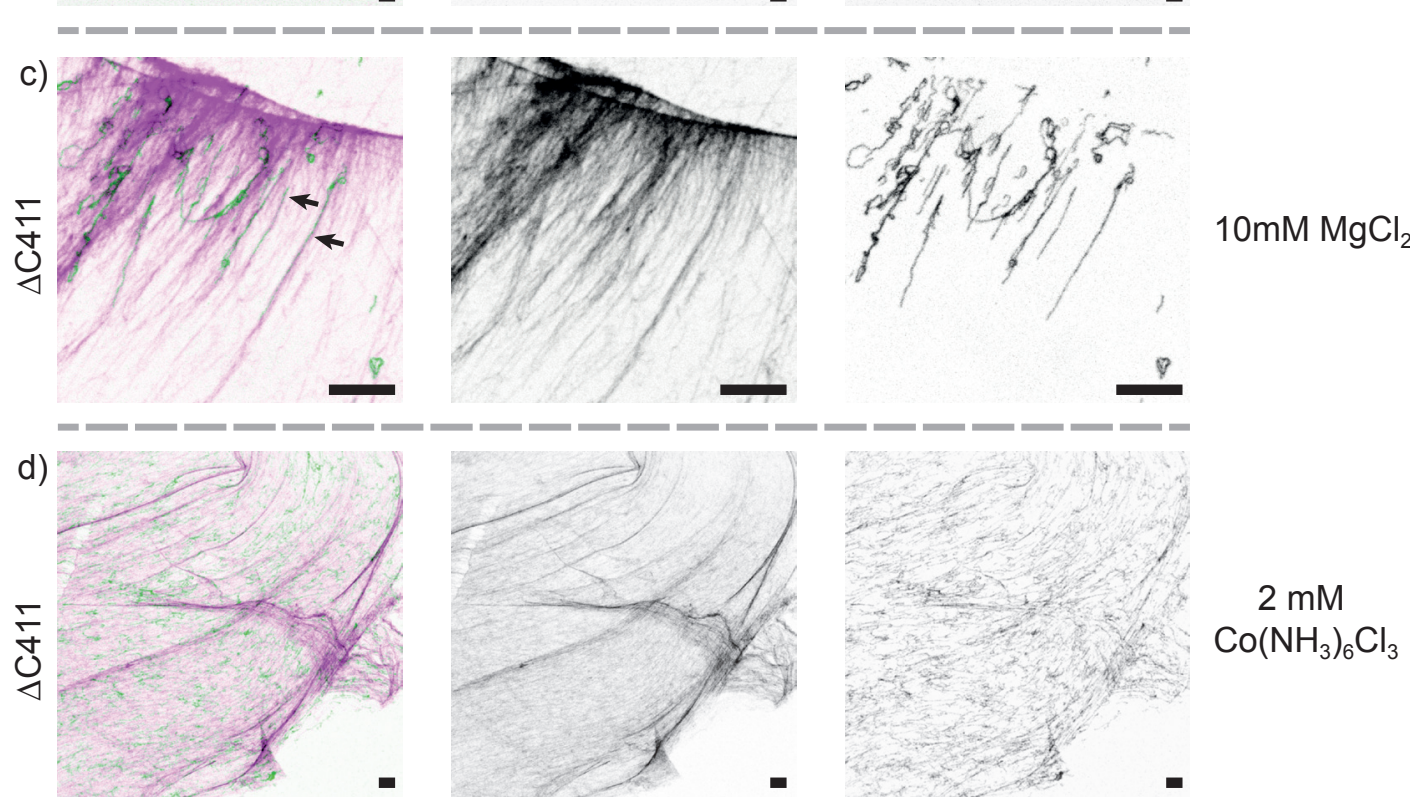

Figure 7.14: Interaction of vimentin with vimentin mutants in the presence of multivalent ions. The tail of the vimentin mutant $\Delta \mathrm{C} 411(\Delta \mathrm{C} 455)$ is truncated and lacks the last 55 (11) amino acids compared to vimentin wild-type. The merged channel images in a)-d) directly indicate the high correlation in the network consisting of vimentin (purple) and the vimentin mutants (green). The arrows show exemplary positions at which a direct overlap between vimentin and the mutant is visible. The integration of the mutants is obvious for the presence of magnesium (a-c), as well as for hexammine cobalt (d). In the periphery next to the network aggregates ( $b$ and $d$ ) there are almost no filaments. This indicates the same affinity of the mutants to be integrated into the vimentin network as the vimentin filaments themselves. All scale bars are $10 \mu \mathrm{m}$. 
The mutants are part of the networks in the presence of both magnesium ions as well hexammine chloride. Next to the larger network aggregates (Fig. 7.14b/c) there are neither vimentin filaments nor filaments of the mutants. Consequently, the affinity of both proteins is the same to integrate into the network. The conclusion from these experiments is that the filaments of vimentin mutants, which (partly) lack their tail, are also integrated into the emerging networks. A difference between the mutants $\Delta C 411$ and $\Delta C 455$ is not observed. This aspect is further investigated in Sec. 8.6 and discussed in Sec. 9.2.4. 



\section{8 \\ Polyelectrolyte nature of vimentin}

\subsection{System parameter}

Here, the electrostatic properties of vimentin filaments in the drops are discussed. An important parameter is the charge of vimentin, which is a prerequisite for the discussion of vimentin as a polyelectrolyte. For this purpose, we obtain an approximation of the charge of vimentin by analyzing the acidic and basic groups in the amino acid sequence of vimentin. [24] Dissociation of an acidic group $A$ is described by $\mathrm{HA} \rightleftharpoons \mathrm{H}^{+}+\mathrm{A}^{-}$. The degree $\tau=\left[\mathrm{A}^{-}\right] /[\mathrm{A}]$ of dissociation is given by the Henderson-Hasselbalch equation, considering the total concentration $[\mathrm{A}]=$ $\left[\mathrm{A}^{-}\right]+[\mathrm{HA}][78]$ :

$$
p \mathrm{H}=p \mathrm{~K}_{\mathrm{a}}+\log _{10} \frac{\tau}{1-\tau} .
$$

Based on this equation and the $p \mathrm{~K}_{\mathrm{a}}$-values of each acidic or basic amino acid, the $\mathrm{N}$ - and the C-terminus of the protein, the (partial) charges of the groups are determined at $p \mathrm{H}=7.5 .^{1}$ It has to be pointed out that this procedure constitutes only an approximation to the charge of the protein at each amino acid side, since the real $p \mathrm{~K}_{\mathrm{a}}$-values depend on the local environment of an amino acid. [31,129] The resulting approximation for the average charge of a vimentin monomer (amino acid sequence in Sec. 3.1.1) is given in Fig. 8.1. Analyzing all charges gives a total

\footnotetext{
${ }^{1}$ Side-chain polarity, charge and $p \mathrm{~K}_{\mathrm{a}}$-values of the acidic or basic groups are: cysteine: acidic, $-e, 8.3$; histidine: basic, $+e, 6.0$; aspartic acid and glutamic acid: acidic, $-e, 4.1$; tyrosine: acidic, $-e, 10.9$; lysine: basic, $+e, 10.8$; arginine: basic, $+e, 12.5$; N-terminus: basic, $+e, 8.0$; C-terminus: acidic, $-e, 3.1$. [19]
} 


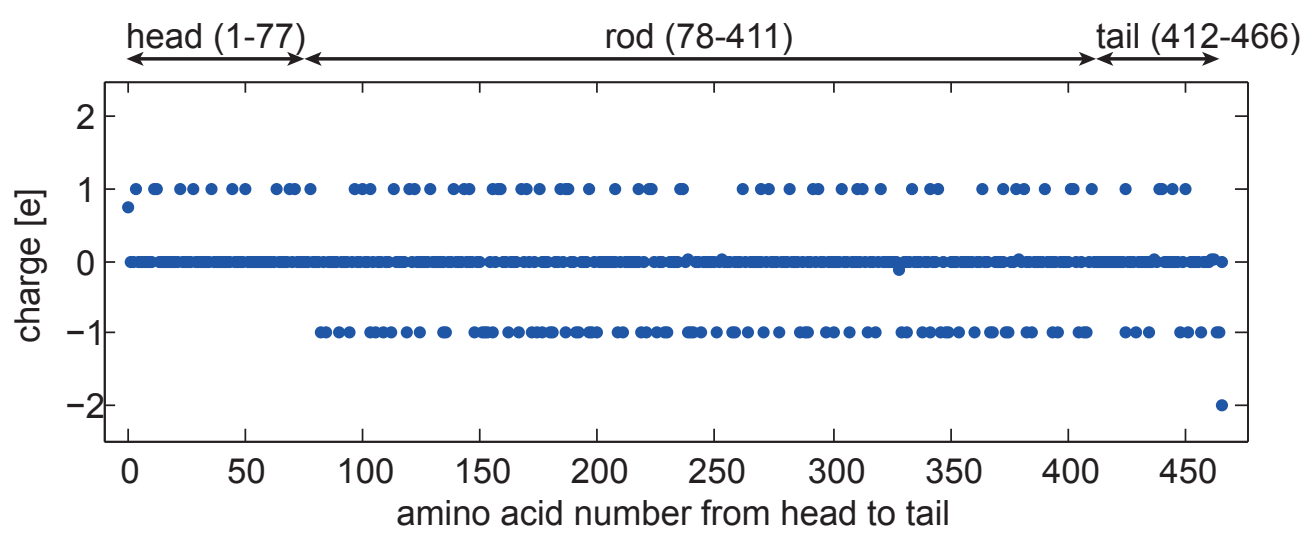

Figure 8.1: Charges along the amino acid sequence of vimentin. Approximation of the averaged line charge of the wild-type vimentin monomer $(p \mathrm{H}=7.5)$. The net charge is $-19 e$.

net charge of the vimentin monomer ${ }^{2}$ :

$$
q_{\mathrm{vim}}=-19 e .
$$

Further, it has to be pointed out that all charges in the head region are positive (the net charge is $+12 \mathrm{e}$ ). These charges are part of the interaction between two vimentin dimers. [117] The net charge of the tail region is $-5 e$.

Taking into account that a ULF consists of 32 monomers and the fact that each ULF takes $43 \mathrm{~nm}$ in the mature filament [121], the average linear line charge $\lambda$ of a mature vimentin filament with 32 monomers in cross-section is

$$
\lambda=-14 \frac{e}{\mathrm{~nm}} .
$$

This leads to charge spacing $b$ and charge density parameter $\xi$ of (Sec. 2.2.2)

$$
\begin{aligned}
& b=0.0714 \mathrm{~nm}, \\
& \xi=9.9 .
\end{aligned}
$$

Under the assumption that all the net charge of vimentin contributes to the surface charge of vimentin (modeled as a cylinder with radius $R=5 \mathrm{~nm}$, see Sec. 2.2.2) the surface charge is (Eq. 2.16)

$$
\sigma=0.071 \mathrm{C} \mathrm{m}^{-2} \text {. }
$$

\footnotetext{
${ }^{2}$ In a recent publication this value is also found. [60]
} 
For the interaction of a polyelectrolyte and ions in the solution, three length scales are important, since they determine the counterion competition regime [105]:

- The Debye length (Eq. 2.8) in the range of (Tab. 8.2) $l_{\mathrm{D}}=0.77 \ldots 0.96 \mathrm{~nm}$.

- The average distance $l_{\lambda, z}$ of a z-valent ion (energy $k_{\mathrm{B}} T$ ) [105] from a charged surface which is half the Gouy-Chapman length (Eq. 2.21): $l_{\lambda, z}=0.25 \mathrm{~nm} / z \leq 0.25 \mathrm{~nm}$ for all valencies $z$.

- The polyelectrolyte radius $R=5 \mathrm{~nm}$ in comparison to $l_{\mathrm{D}}, l_{\lambda, z}$ shows how planar the surface of vimentin appears (Sec. 2.2.2).

The relation $l_{\lambda, z}<l_{\mathrm{D}}$ implies that the nonlinear Poisson-Boltzmann Eq. is important for the process, as its decay $l_{\lambda, z}$ is smaller than the length scale found with the linear Poisson-Boltzmann equation as represented by $l_{\mathrm{D}}$ (Debye-Hückel approximation). Additionally, both $l_{\lambda, z}$ and $l_{\mathrm{D}}$ are much smaller than the vimentin radius $R$. Therefore, the surface of vimentin has to be considered as planar. [105].

\subsection{Manning Counterion condensation}

When applying the theory of Manning (Sec. 2.2.2) to our system, it is found that $\xi$ is clearly higher than 1 and therefore counterion condensation is expected (Sec. 2.2.2). In comparison to F-actin and DNA, the $\xi$-value of vimentin filaments is higher, and lower compared to MTs (see Tab. 2.3). In Tab. 8.1 the fraction $\Theta$ of the vimentin filament charge compensated in the presence of only one bound counterion type with valency $z=1,2,3,4$ is shown. Already the counterions with minimal valency $z=1$ are expected to neutralize approximately $90 \%$ of the

\begin{tabular}{llllll}
\hline biopolymer & $\xi$ & $\Theta_{z=1}$ & $\Theta_{z=2}$ & $\Theta_{z=3}$ & $\Theta_{z=4}$ \\
\hline human vimentin & 9.9 & 0.9 & 0.95 & 0.97 & 0.97 \\
\hline
\end{tabular}

Table 8.1: Vimentin considered as 'Manning polyelectrolyte'. Vimentin discussed in Manning counterion condensation theory (Sec. 2.2.2). In analogy to Tab. 2.3, we give the fraction $\Theta$ of vimentin charges that is compensated in the presence of ions with valency $z$. Note that this concept considers each ion species separately and competition between ions of different valency is neglected. 
charge of vimentin filaments. This result is expected as we find in Sec. 8.1 that the surface of vimentin has to be considered as planar. The charge of planar surfaces is always neutralized (Sec. 2.2.2 and [90]) and this explains why the $\Theta$ values are almost 1 for any counterion valency. This is similar to microtubules (Tab. 2.3).

\subsection{Competitive ion condensation}

Potassium chloride is needed to initiate the assembly of vimentin to filaments and to stabilize them later on $\left(c_{\mathrm{KCl}}=100 \mathrm{mM}\right)$. In the phosphate buffer $(2 \mathrm{mM})$, sodium ions are present as well, which we neglect in the following as the likewise monovalent potassium ions dominate the contribution of ions with this valency (about 50 times higher in concentration). Secondly, multivalent ions are added to our system when all aqueous components are encapsulated in the drops. This provides us with a system in which we have two types of counterions of different concentration that are in competition for the condensation onto the oppositely charged vimentin surface. In the following we investigate this competitive binding quantitatively.

The competition of counterions is investigated following the concept of Rouzina and Bloomfield (Sec. 2.2.3 and [107]). For this concept we use the counterion competition regime for the nonlinear Poisson-Boltzmann equation in planar geometry (Sec. 8.1). Following Sec. 2.2.3 and 8.1, we obtain the fundamental quantities of our system as shown in Tab. 8.2. Based on these quantities, we apply the model

\begin{tabular}{crl}
\hline quantity & & value \\
\hline $\mathrm{KCl}$ concentration & $c_{\mathrm{KCl}}$ & $=100 \mathrm{mM}$ \\
$\mathrm{Mg}^{2+}$ concentration range & $c_{\mathrm{Mg}^{2+}}$ & $=0 \ldots 18.6 \mathrm{mM}$ \\
$\mathrm{Co}\left(\mathrm{NH}_{3}\right)_{6}^{3+}$ conc. range & $c_{\mathrm{Co}\left(\mathrm{NH}_{3}\right)_{6}^{3+}}=0 \ldots 9.6 \mathrm{mM}$ \\
charge spacing & $b$ & $=0.0714 \mathrm{~nm}$ \\
filament radius [97] & $R$ & $=5 \mathrm{~nm}$ \\
ionic strength & $I$ & $=0.100 \ldots 0.156 \mathrm{M}$ \\
& & $(0.100 \ldots 0.158 \mathrm{M})$ \\
Debye length & $l_{\mathrm{D}}=0.96 \ldots 0.77 \mathrm{~nm}$ \\
surface charge & & $(0.96 \ldots 0.77 \mathrm{~nm})$ \\
surface concentration of counterions & $\sigma$ & $=0.071 \mathrm{C} \mathrm{m}{ }^{-2}$ \\
& $n_{\mathrm{s}}$ & $=1.47 \mathrm{M}$ \\
\hline
\end{tabular}

Table 8.2: System parameter for polyelectrolyte analysis. Values in brackets apply for all cases where $\mathrm{Co}\left(\mathrm{NH}_{3}\right)_{6}^{3+}$ is used instead of $\mathrm{Mg}^{2+}$. 
a)

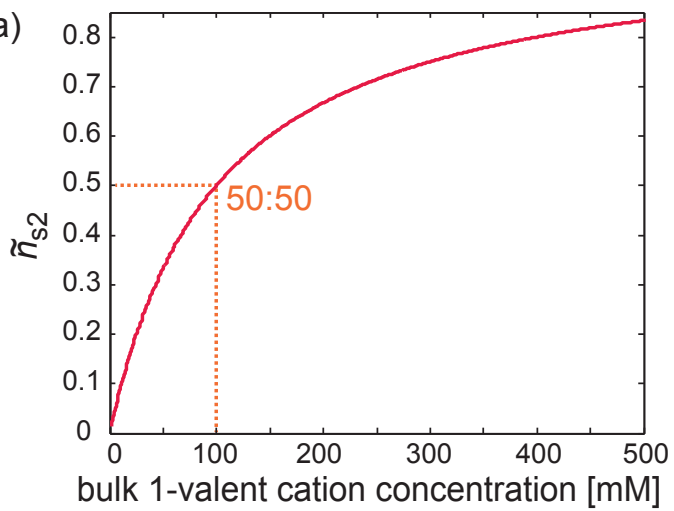

c)

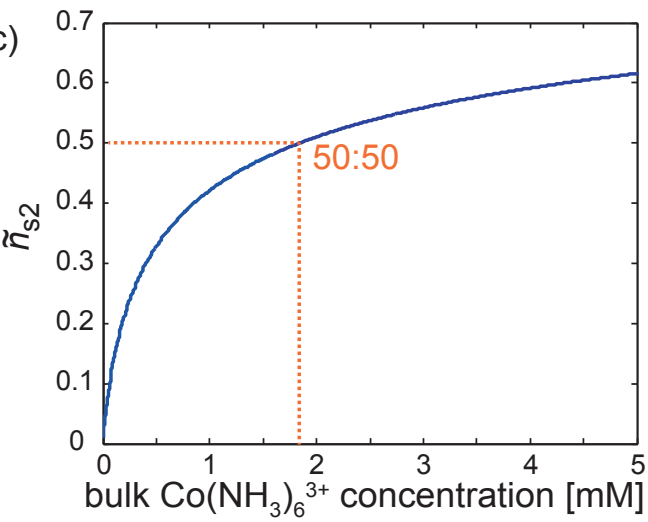

e)

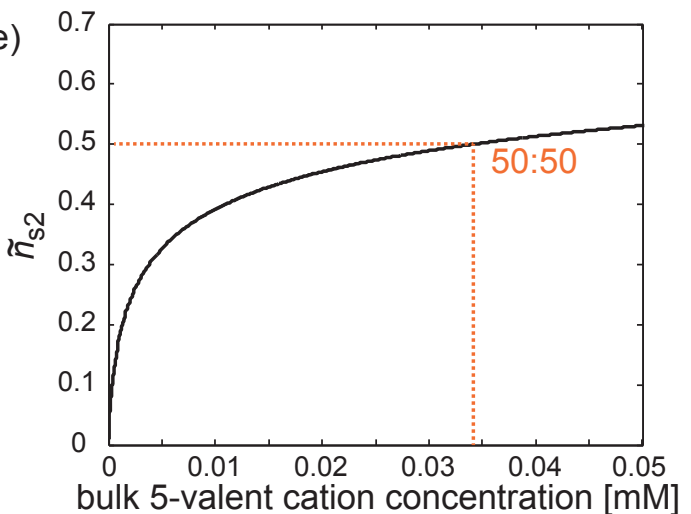

b)

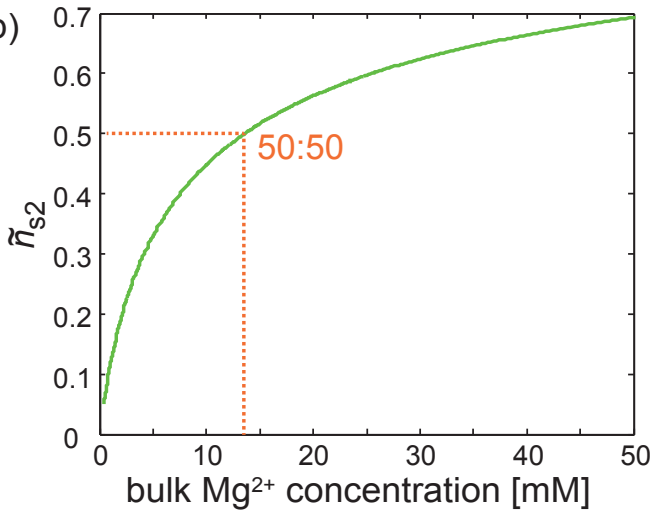

d)
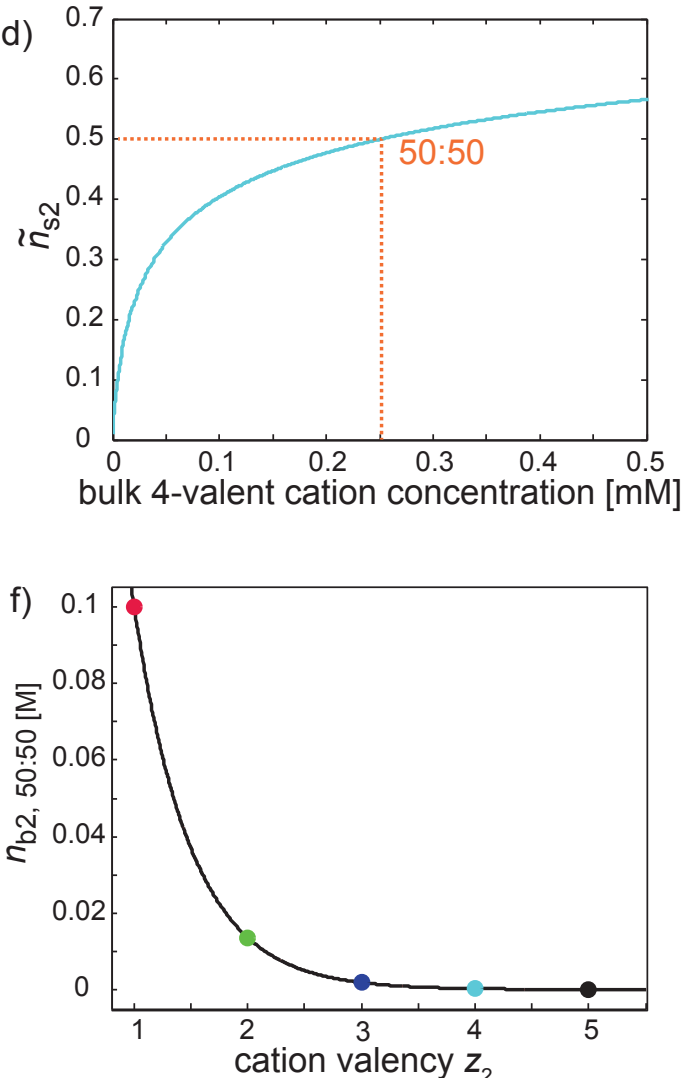

Figure 8.2: Quantification of ion competition on the surface of vimentin. The calculated fraction $\tilde{n}_{\mathrm{s} 2}$ of multivalent ions in competitive binding with $100 \mathrm{mM} \mathrm{KCl}$ (bulk concentration) for the most important bulk concentrations of 1 to 5 -valent counterions (ae). Note that the x-scale changes from figure to figure by one order of magnitude (a-e). In f) we give the bulk concentration $n_{\mathrm{b} 2,50: 50}$ at which the second cation has the same fraction as the monovalent potassium ions. They follow a power law (Eq. 8.7).

given by Rouzina and Bloomfield as sketched in Sec. 2.2.3. Since the system variables are quantifiable here, the details behind the general parameter $Y$ (Eq. 2.20) are known and we 'translate' Fig. 2.7 to our system. In Fig. 8.2a-e, we compare 
the fraction $\tilde{n}_{\mathrm{s} 2}$ of cations of valency one to five that are in competitive binding to the potassium ions, which have a constant bulk concentration of $100 \mathrm{mM}$. This fraction $\tilde{n}_{\mathrm{s} 2}$ changes as the bulk concentration of the corresponding cation changes while the bulk concentration of potassium is constant. The di- and trivalent cations are of special interest here, since ions of these valencies were used in our experiments (magnesium ions $\mathrm{Mg}^{2+}$ and hexammine cobalt ions $\mathrm{Co}\left(\mathrm{NH}_{3}\right)_{6}^{3+}$ ). An important point is the bulk concentration $n_{\mathrm{b} 2,50: 50}$ at which the fraction of the monovalent and the $z_{2}$-valent competitors have equal concentrations on the filament. Rearrangement of Eq. 2.20 with $\tilde{n}_{\mathrm{s} 1}=\tilde{n}_{\mathrm{s} 2}=0.5$ and $z_{1}=1$ reveals that $n_{\mathrm{b} 2,50: 50}$ follows a function of the valency $z_{2}$ of the multivalent ion species (Fig. $8.2 f)$ :

$$
n_{\mathrm{b} 2,50: 50}=\frac{n_{\mathrm{s}}}{2} \times\left(\frac{2 n_{\mathrm{b} 1}}{n_{\mathrm{s}}}\right)^{z_{2}}=0.736 \mathrm{M} \times 0.136^{z_{2}} .
$$

Since 0.136 is close to 0.1 this roughly means that when the valency of the second competitive cation rises by one, the 50:50 share is reached one order of magnitude lower. We point out that

$$
n_{\mathrm{b} 2,50: 50}=\left\{\begin{array}{ll}
0.0136 \mathrm{M} & \text { for } \mathrm{Mg}^{2+} \\
0.0019 \mathrm{M} & \text { for } \mathrm{Co}\left(\mathrm{NH}_{3}\right)_{6}^{3+}
\end{array} .\right.
$$

We use these findings in the interpretation of experimental results in Sec. 9.2.2. In the following, ' $n_{\mathrm{b} 2,50: 50}$ ' is a quantity that gives the order of the bulk concentration of the multivalent ions, at which the fraction of these ions becomes comparable on the filaments, as then the number of the potassium and multivalent ions on the filament is equal. However, the 50:50 fraction on the filament is just a selected point and is considered as orientation for surface concentrations of the multivalent ions.

At the threshold concentrations we find in the experiments for the onset of aggregation (chapter 7), the fraction $\tilde{n}_{\mathrm{s} 2}$ of multivalent ions (Sec. 2.2.3) on the surface of the polyelectrolyte is

$$
\tilde{n}_{\mathrm{s} 2}=\left\{\begin{array}{ll}
45 \% & \text { for } 10 \mathrm{mM} \mathrm{Mg}^{2+} \\
33-53 \% & \text { for } 0.5-2.4 \mathrm{mM} \mathrm{Co}\left(\mathrm{NH}_{3}\right)_{6}^{3+}
\end{array} .\right.
$$


Consequently, $\tilde{n}_{\mathrm{s} 2}$ takes approximately the same values at the observed threshold concentration for both the divalent as well as the trivalent counterions. This is a main finding in the analysis of the competitive binding of potassium and multivalent ions. This aspect is further discussed in Sec. 9.2.2.

\subsection{Robustness of the analysis}

It is of interest whether and to which extent our quantitative analysis (Sec. 8.3) of the polyelectrolyte nature of vimentin is robust towards inaccuracies. The surface charge $\sigma$ of vimentin filaments is an important quantity for this discussion. It is connected to the radius of the filaments as well as the spacing of charges (Eq. 2.16). In our calculation the average of 32 protein monomers in the cross section of the filament is considered (diameter $10 \mathrm{~nm}$ ). However, a polymorphism of the filament shape is reported. Along the filament there can be more or fewer than the average of 32 monomers in one cross section. [52] Locally this leads to a deviation of the filament diameter as well as charge. The outcome of this consideration is shown in Tab. 8.3. This consideration gives the 'deviation surface concentration' $n_{\mathrm{s}, \mathrm{dev}}$ of counterions. In the limiting cases, we find $n_{\mathrm{s}, \mathrm{dev}}=0.5 n_{\mathrm{s}}$ to $n_{\mathrm{s}, \mathrm{dev}}=1.6 n_{\mathrm{s}}$. Herein, $n_{\mathrm{s}}$ is the surface concentration of counterions as considered in the calculation above, which assumes the average of 32 monomers at a filament diameter of $10 \mathrm{~nm}$. The lower bound is given by the uncertainty in the filament diameter for filament segments of 30 monomers. We consider a literature value for the surface charge of vimentin. [139] However, the derivation

\begin{tabular}{llllll}
\hline$N$ & $\mathbf{D}[\mathbf{n m}]$ & $\sigma\left[10^{-2} \mathbf{C ~ m}^{-2}\right]$ & $n_{\mathbf{s}, \mathbf{d e v}}[\mathbf{M}]$ & $n_{\mathbf{s}, \mathbf{d e v}}\left[n_{\mathbf{s}}\right]$ & $n_{\mathbf{b 2}, \mathbf{5 0 : 5 0}}[\mathbf{m M}]$ \\
\hline 32 & 10 & 7.1 & 1.5 & 1 & 14 \\
30 & $9.8 \pm 1.0$ & $6.9 \pm 1.4$ & $1.4 \pm 0.6$ & $0.9 \pm 0.4$ & $27-10$ \\
37 & $10.7 \pm 1.1$ & $7.8 \pm 1.6$ & $1.8 \pm 0.7$ & $1.2 \pm 0.5$ & $19-8$ \\
45 & $11.6 \pm 0.8$ & $8.7 \pm 1.2$ & $2.2 \pm 0.6$ & $1.5 \pm 0.4$ & $12-7$ \\
$32^{\dagger}$ & - & 9.1 & 2.4 & 1.6 & 8 \\
\hline
\end{tabular}

Table 8.3: Deviations in surface charge density. From the number $N$ of vimentin monomers per cross-section and the diameter $(D)$ of the corresponding filaments [52], we determine the concentration $n_{\mathrm{s} \text {, dev }}$ of counterions on the surface of vimentin in units of the average surface concentration $n_{\mathrm{s}}$ (Sec. 8.3). Additionally, the values in the last row $(\dagger)$ give a literature value for the surface charge of vimentin. [139]. The $n_{\mathrm{b} 2,50: 50 \text {-value is }}$ calculated for a divalent ion $\left(z_{2}=2\right)$. 
of this literature value is not fully comprehensible. Therefore, we consider the corresponding value as an upper bound. The impact of a different surface concentration of counterions is illustrated in Fig. 8.3. Regardless of the reason for a change in the surface charge $\sigma$, the meaning of the change can be understood by the analysis of Eq. 8.7. At fixed bulk concentration $n_{\mathrm{b} 1}=c_{\mathrm{KCl}}=100 \mathrm{mM}$, it follows that

$$
n_{\mathrm{b} 2,50: 50}=\frac{\left(2 n_{\mathrm{b} 1}\right)^{z_{2}}}{2} \times n_{\mathrm{s}}^{1-z_{2}}
$$

Importantly, for relevant deviations, i.e. when $n_{\mathrm{s} \text {, dev }}$ is close to $n_{\mathrm{s}}$, the changes in $n_{\mathrm{b} 2,50: 50}$ are not too large. The order of magnitude of $n_{\mathrm{b} 2,50: 50}$ changes only substantially , if $n_{\mathrm{s} \text {, dev }}$ gets markedly smaller. Despite the polymorphism occurrence, it must not be forgotten that the common value of monomers is 32. [97] Therefore, our calculations performed above are justified. For the literature value (Tab. 8.3) the calculation gives $n_{\mathrm{b} 2,50: 50}=8 \mathrm{mM}$. Consequently, also for this value the order of magnitude is still the same as for our calculation (Eq. 8.8).

In our analysis, we neglected the contribution of the monovalent sodium ions from the buffer, since the theory we apply considers only one species of mono-

a)

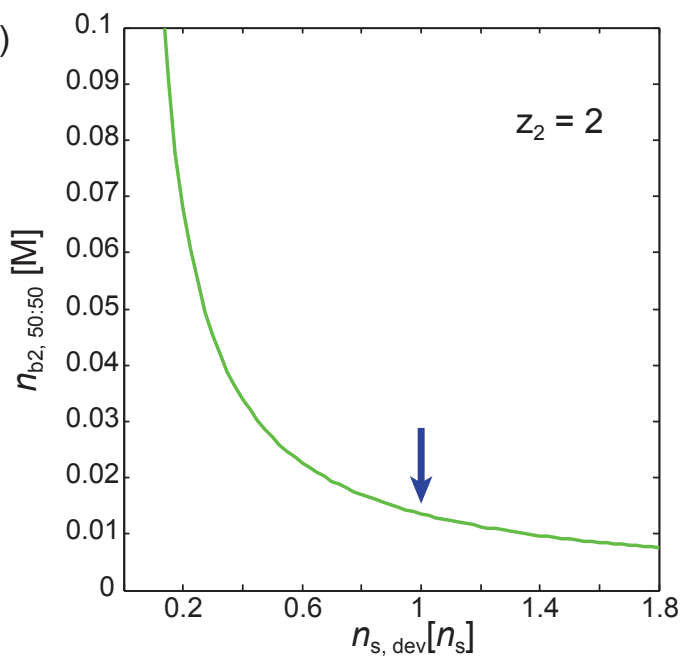

b)

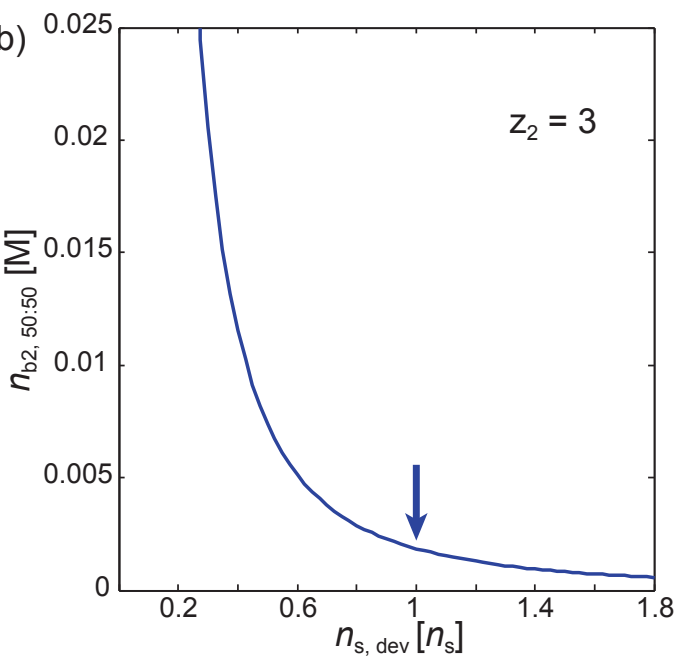

Figure 8.3: The role of the surface charges. The effective counterion surface charge concentration $n_{\mathrm{s} \text {, dev }}$ is given in units of the calculated surface charge concentration $n_{\mathrm{s}}$ of vimentin for $c_{\mathrm{KCl}}=100 \mathrm{mM}$ that is in competition with a divalent (a) or a trivalent ion (b). The change in the surface concentration shifts the bulk concentration of the multivalent ion $\left(n_{\mathrm{b} 2}, 50: 50\right)$ at which it has the same surface charge concentration as the $\mathrm{KCl}$ ions. In proximity to $n_{\mathrm{s} \text {, eff }}=1$, the shift in $n_{\mathrm{b} 2,50: 50}$ is in both cases comparatively small. 
valent ions. The influence of the sodium ions is expected to be very small, as the presence of $100 \mathrm{mM}$ potassium chloride outnumbers the sodium ions. Additionally, small drop volume changes due to drop growth or shrinkage are present in the time course of the experiment (Sec. 5.2). This changes the bulk concentration of both potassium and multivalent ions slightly. Although the ratio of the concentrations remains the same during drop volume changes $\left(n_{\mathrm{b} 1 \text {, start }} / n_{\mathrm{b} 2 \text {, start }}=\right.$ $\left.n_{\mathrm{b} 1 \text {, later }} / n_{\mathrm{b} 2 \text {, later }}\right)$, the competitions of counterions changes a bit. This can be seen by rearranging Eq. 8.7:

$$
\frac{n_{\mathrm{b} 2,50: 50, \text { start }}}{n_{\mathrm{b} 2,50: 50, \text { later }}}=\left(\frac{n_{\mathrm{b} 1, \text { start }}}{n_{\mathrm{b} 1, \text { later }}}\right)^{z_{2}}=\left(\frac{n_{\mathrm{b} 2, \text { start }}}{n_{\mathrm{b} 2, \text { later }}}\right)^{z_{2}} \neq \frac{n_{\mathrm{b} 2, \text { start }}}{n_{\mathrm{b} 2, \text { later }}} .
$$

For example, when a drop with $c_{\mathrm{Mg}^{2+}}=10 \mathrm{mM}$ shrinks over time and has $c_{\mathrm{Mg}^{2+}}=$ $10.64 \mathrm{mM}$ later on (as it is the case in Sec. 5.2), we find $n_{\mathrm{b} 2,50: 50 \text {, later }} / n_{\mathrm{b} 2,50: 50 \text {, start }}=1.13$ or $n_{\mathrm{b} 2,50: 50, \text { later }}=0.0154 \mathrm{M}$. Consequently, the effect of drop volume changes are of the same quantity as effects of the polymorphism (Tab. 8.3).

\subsection{Strength of counterion correlation}

Based on the theoretical background presented in Sec. 2.2.4, we quantify the correlation strength between counterions on the surface of vimentin by means of the coupling parameter $\Xi$ as defined by Eq. 2.23. We present the values in Tab. 8.4, where we also show data for DNA (taken from [90]) and calculations for F-actin. DNA has twice the surface charge of vimentin and as $\Xi \propto \sigma \times z^{3}$, the correlation parameter is twice as large for the corresponding valency $z$ in comparison to vimentin. For both systems, the counterions of valency 1 couple with a strength $\Xi$ that is close to 1 and therefore they are weakly coupling. For all higher valencies $\Xi>10$. Naji et al. find that this coupling strength can be sufficient to mediate attraction between two polyelectrolytes (Sec. 2.2.4).

However, DNA does not condense in aqueous solutions in the presence of counterions of valency 2 (Sec. 2.1.3). Consequently, this criterion taken alone is not sufficient, likely because the resulting attraction mediated by ion correlation is not strong enough. For vimentin, we find attraction in the presence of diand trivalent ions (Sec. 7). Our interpretation is that the number of strongly correlating multivalent ions on the surface of the vimentin filaments is high enough, once a bulk threshold concentration of the magnesium ions is exceeded. Then, 


\begin{tabular}{cccccc}
\hline biopolymer & $\sigma\left[\mathrm{Cm}^{-2}\right]$ & $R[\mathrm{~nm}]$ & $z$ & $\mu[\mathrm{nm}]$ & $\Xi$ \\
\hline Human vimentin & 0.071 & 5 & 1 & 0.50 & 1.4 \\
& & & 2 & 0.25 & 11.3 \\
& & & 3 & 0.17 & 38.1 \\
& & & 4 & 0.13 & 90.3 \\
DNA [90] & \multirow{2}{*}{0.144} & 1 & 1 & 0.24 & 2.8 \\
& & & 2 & 0.12 & 22.4 \\
& & & 3 & 0.08 & 75.6 \\
& & & 4 & 0.06 & 179 \\
F-actin & \multirow{2}{*}{0.0291} & 3.5 & 1 & 1.23 & 0.6 \\
& & & 2 & 0.62 & 5 \\
& & & 3 & 0.41 & 16 \\
& & & 4 & 0.31 & 37 \\
\hline
\end{tabular}

Table 8.4: Coupling parameter of ions for different biopolymers. Comparison of vimentin, DNA and F-actin in terms of their surface charge $\sigma$, radius $R$, and GouyChapman length $\mu$ as well as coupling parameter $\Xi$ for different valencies $z$. Data for DNA are from [90]. Data for F-actin are calculated with the filament radius and charge separations along the filament as found in [131].

the resulting force - mediated by magnesium ions that correlate strongly-is high enough to mediate filament-filament attraction. Analogously, the same holds for the trivalent hexammine cobalt ions. Details on this hypothesis are discussed in Sec. 9.2.2. Additionally, we provide the coupling parameter for F-actin in Tab. 8.4. For F-actin, $\Xi$ is lower for divalent ions in comparison to vimentin and DNA. Nevertheless, attraction of F-actin is observed in the presence of divalent ions. $[57,131]$

\subsection{The tail of vimentin}

Lin et al. performed rheology experiments on vimentin and hypothesized that the last 11 residues of the tail of vimentin are the most important interaction domain for divalent ions. [76] We consider this aspect within the context of the polyelectrolyte nature of vimentin. Vimentin has two flexible side arms (Fig. 2.4). The head region is strongly integrated into the tetramer of vimentin. [51] Therefore, only the tail can actually play an important role for inter-filament attraction in the presence of multivalent ions. 
Note that in the literature, the first amino acid of the tail is not consistently defined and here we discuss the amino acids 412 to 466 as the 'tail' (Sec. 3.1.1). We consider a short and a long isolated part of the tail region, i.e. amino acids from 456 to 466 and from 412 to 466 . Both segments have a net charge of $\approx-5 e$ (Fig. 8.1 ) and a contour length of approximately $1.5 \mathrm{~nm}$ and $7.2 \mathrm{~nm}$, respectively. Consequently, the prerequisite for an attraction mediated by positive ions is given by this net negative charge.

To begin with, we assume a rod-like form for the tail fragments. In this case, the Manning parameter is $\xi=0.5$ for long fragment of the tail and $\xi=1.9$ for the short part (Eq. 2.12). This calculation is valid when the sidearm is elongated linearly. Considering Eq. 2.15, it is expected that monovalent (divalent) counterions neutralize $47 \%$ (74\%) of the charge of the short part of the tail. For the long part of the tail no neutralization is expected, as $\xi=0.5$ is too small for both mono- and divalent ions (Eq. 2.14). However, a discussion of the competitive binding of monoand divalent ions on parts of the tail is most probably error-prone, as the tail is highly flexible and therefore an assumption of a rod is likely to be not justified. Consequently, the applied Manning theory might not hold for the tail fragments. In addition, the diameters of the tail fragments are also not accessible, because it is a single amino acid chain. Therefore, the attribution of a surface charge to the flexible tail is challenging.

We consider that the net charges add up for the whole vimentin filament. When we separate the charge contribution of the tail from the rest of the vimentin monomer (amino acids 1-411), we still obtain a net charge of $-14 \mathrm{e}$ for the rest of the protein monomer. As the tail-truncated vimentin still forms filaments (Sec. 7.4.2), we calculate the Manning parameter of the filaments that are built of tailtruncated monomers: $\xi=7.4$. This calculation is the same for both mutants of the tail-truncated vimentin. Therefore, the tail-truncated vimentin mutants have a pronounced electrostatic character also without (parts) of the tail. It is still very relevant for counterion condensation and competitive binding as the surface charge of tailless vimentin is $\sigma=0.05 \mathrm{C} \mathrm{m}^{-2}$ and therefore still larger than the surface charge of F-actin (Tab. 8.4).

In conclusion, electrostatic effects of this (partly) tailless vimentin are present. It cannot be excluded that the tail plays an important role for attraction like it is observed for neurofilaments. [16] However, when the head-rod complex of vimentin is considered alone, it has a distinct polyelectrolyte character and there- 
8 POLYELECTROLYTE NATURE OF VIMENTIN

fore is important for the interaction between vimentin filaments. This aspect is further discussed in a broader context in Sec. 9.2.4. 


\section{Discussion}

\subsection{Microfluidic techniques}

The microfluidic devices $\mathrm{C}$ and $\mathrm{T}$ were used to answer specific questions on the interaction of vimentin and multivalent ions. Here, we evaluate the overall performance of these devices also with respect to general challenges in protein research as well as alternative methods.

\subsubsection{The benefit of device $C$}

Using device C, we successfully test the dependence of vimentin filament aggregation on magnesium ion concentration. In these studies, we obtain data from about 250 drops per experiment. Due to the conceptual idea of the experiment, the contents of the drops are directly comparable.

A possible alternative microfluidic method could be realized using the device from Schmitz et al. [109] In their device a multitude of drops all sharing the same chemical composition (e.g. magnesium ion concentration) can be stored and studied in constrictions. For an optimal trapping of the drops, the flow has to be turned off. [109] For vimentin, a stopped flow results most probably in clogging of the channels in the drop production region (Sec. 5.3). Consequently, one device can only be used to study the aggregation of vimentin at a single concentration (Sec. 5.3 and 9.1.5). For the next study of the aggregation of vimentin at another salt concentration, a new device has to be used. The result is that the experiment has to be performed several times to collect data for a set of different drop compositions. 
In our device, the observation of vimentin filament aggregation at varying magnesium ion concentrations is achieved in a single experiment. At the same time, our method provides a high degree of comparability concerning the preparation of the networks prior to imaging. All drops are produced within one minute in the same device and then stored in the same row of constrictions. This concept is very reliable.

Additionally, only a very small sample volume is needed to obtain data for 250 drops of tunable composition. From the startup of the device to the point where all drops are stored in the drop storage, a minimum amount of $20 \mu \mathrm{L}$ is needed, when the experiments are carried out optimally. This very low sample consumption is important for precious samples which are hard to obtain and large amounts of the sample are not available. As the concept of the microfluidic device is rather universal, those precious solutions can also be studied in the device $\mathrm{C}$ and examined for their interactions with chemicals.

An alternative for the creation of chemical gradients in drop trapping arrays was published by Sun et al. after we had established the device $C$ as a tool for vimentin research in our lab. [126] This method is based on the trapping of drops in an array. The trapped drops get transiently in contact with another drop. A chemical is exchanged during this contact so that the concentration of that chemical in the drop is tunable. The technique by Sun et al. has no obvious advantage compared to the device $C$ for the experiments we carried out with vimentin. One drawback of their technique is that mixing of the drop content is achieved only by diffusion. However, for vimentin a fast mixing as achieved in the moving-plug like drops (Sec. 5.1) is essential due to mismatch of the diffusion constants of ions and protein filaments (Sec. 9.1.4). Nevertheless, the device of Sun et al. enables a new type of experiments that cannot be performed with device C: Vimentin filaments could be encapsulated into drops at a magnesium concentration above the threshold concentration for aggregation. Consequently, vimentin networks would form. Subsequently, the concentration of magnesium could be decreased in the drops again with the technique of Sun et al. This way, it could be tested whether the network formation is reversible, as the magnesium concentration is below the threshold at some point. 


\subsubsection{The benefit of device $T$}

Studying the interaction of two substances can be a tedious task, when their interaction takes place shortly after these substances are mixed. These experiments have at least two requirements: Firstly, fast mixing between the two substances is needed. This way the mixing process is not overlaid significantly to the interaction process. Secondly, the content has to be imaged rapidly after the first contact between the two substances. The features at the beginning of the interaction process might be otherwise not observable. Additionally, there is often the need to image the interaction for some time, i.e. minutes to hours, to capture the intermediate states until the system is equilibrated. Our study of the interaction of vimentin with multivalent salt meets these requirements.

A straightforward approach to investigate time dependent filament aggregation is to mix assembled vimentin and the salt solution in a test tube. Mixing can be achieved in this test tube (multiple in- and outtake with the pipette), and shortly afterwards, the solution is deposited onto a cover glass. Then, the system is imaged on the microscope. The advantage of this approach is its simplicity. However, this experimental approach has disadvantages. First, the start point of the interaction is not clearly defined. It is also not clear, when the solution is mixed and at which performance, as it is highly improbable that the mixing is done each time with exactly the same mixing scheme (at least as long as a human being accomplishes the task). Secondly, it will most likely take some seconds to deposit the sample accurately on a cover glass and find the right focal plane with the microscope.

Vimentin filaments, in particular, exhibit another challenge: The protein filaments stick preferentially to the surface of chemically untreated cover glasses (Sec. 7.4.1) and become immediately static. A time lapse study on the same filaments is impossible. This is a fundamental drawback compared to the possibilities of the device $\mathrm{T}$. When the protein sticks on the glass surface, the interacting system consists of the glass surface, vimentin and multivalent ions. The reason for this behavior could be the polyelectrolyte nature of vimentin (Sec. 7, 8 and 9.2). For polyelectrolytes, the interaction with charges on surfaces [18] is a phenomenon on its own. [47] Although at least a slight distortion of the results by the cover glasses is expected, vimentin can also interact with the ions prior to the attachment to the glass surface. Consequently, the images on the glass surface are still meaningful. However, for the determination of the persistence length $\mathrm{L}_{p}$ of vimentin, the in- 
teraction with the hard surfaces has influenced the results compared to filaments that are not attached to surfaces (filaments on surface: $\mathrm{L}_{p} \approx 1 \mu \mathrm{m}[87,110]$, nonattached filaments: $\mathrm{L}_{p} \approx 2 \mu \mathrm{m}$ [93]). This example demonstrates the potential influence of an attachment of the filaments to a surface.

In contrast to test tube experiments, the device $\mathrm{T}$ has - like the device $\mathrm{C}$ - generally no problem of protein interaction once the protein is encapsulated in the drops. Instead, for the device $\mathrm{T}$, the aggregation is influenced by the drop internal movement (Sec. 7.3). These internal flows emerge on the basis of the incident oil flow to the trapped drop. They influence the morphology of the vimentin networks as well as the time scale of aggregation. The influence of drop internal flow to networks in the drops is also known for other systems than vimentin. [42] Nevertheless, in our system, the drop internal flows are not a reason for filament attraction, since filament aggregation is not observed in drops that show pronounced drop internal flow in the absence of multivalent ions.

In drop-based microfluidics, the drop internal flows are at least at the very beginning present. Already during the pinch-off mechanism of the drops, drop internal flow is intrinsic. [134] There are also internal flows in the drops when they move with contact to a channel wall. This principle is used in our devices for mixing. Song et al. present a method for time-resolved imaging of a chemical reaction in drops. [118] In their approach the reaction of different solutions starts as soon as the chemicals get in contact in the drops. Then the reaction is followed as the drops are guided through a long channel. Imaging at different positions along the channel captures different time points of the chemical reaction inside the drops. Their system is convincing due to its simplicity and therefore reliability. However, for our studies their system would have important drawbacks. First, the drops that move along a serpentine channel are constantly in contact with the channel walls. Therefore, there is a permanent drop internal flow, which is considerably higher than any flow that occurs in the trap of the device T. Additionally, the content of a resting drop is also considerably easier to image. Images with filament details like in Fig. 7.5 would be very difficult to obtain from drops that move along a channel. For this reason, the device $\mathrm{T}$ is tailored to image vimentin in the drops. The microfluidic concept applied, however, is still general and is likely to prove crucial for the rapid imaging of other (biological) systems that can be encapsulated in drops. 


\subsubsection{Time-stability of salt concentrations}

In an ideal case, the concentrations of the chemicals in the drops do not change over time. However, there are three factors that have an influence on the concentrations over time. In Sec. 5.2, we quantify the presence of drop shrinkage and growth in our system. This quantification shows that the change in concentration of magnesium is negligible. A way to prevent drop shrinkage would be to use water-impermeable device materials (see also Sec. 2.3.2).

In addition to the changes of the chemical concentrations by drop volume changes, there are also transport phenomena between drops. In dependence of the system parameters such as oil, surfactant type, concentration as well as the type of chemicals in the drops, material exchange between drops is reported. For instance, in experiments with drops with a fluorocarbon oil and Krytox-based surfactants, a substantial exchange of the fluorescent dye resorufin is observed. [116] For a drop emulsion system that is comparable to the system we use (Sec. 3.3), Gruner et al. find a significant magnesium ion exchange between neighboring drops on the order of one hour when the magnesium concentration difference between the neighboring drops is initially $200 \mathrm{mM}$ [48]. In the device C, the maximum concentration difference of two neighboring drops is $\approx 1 \mathrm{mM}$. Considering the much smaller difference in the magnesium ion concentration in neighboring drops, the exchange of magnesium should be much smaller than in the experiments of Gruner $e t$ al.

In addition to this estimate that magnesium exchange between neighboring drops in our experiments can be neglected, we analyze our data for any evidence that magnesium ion exchange between the drops affects our results. Once the vimentin filaments have aggregated into a static state in the device $C$, we do not observe any changes of that stage over time. For instance, a direct comparison between drops above the magnesium threshold of $10 \mathrm{mM}$ shortly after the production of the drops and after $405 \mathrm{~min}$ shows no qualitative differences in the networks (Fig. 5.4a/b). If there was a massive transport effect of magnesium, after 405 min the network morphology would also change drastically. Our observation time of the networks starts a few minutes after drop storing and lasts for $\approx 120 \mathrm{~min}$. This range of observation time is due to the imaging sequence we use (Fig. 9.1).

As a result of this imaging technique, our data also contain a 'time axis'. This axis is implied in the plots where the standard deviation $\sigma_{\mathrm{I}}$ of the network mor- 


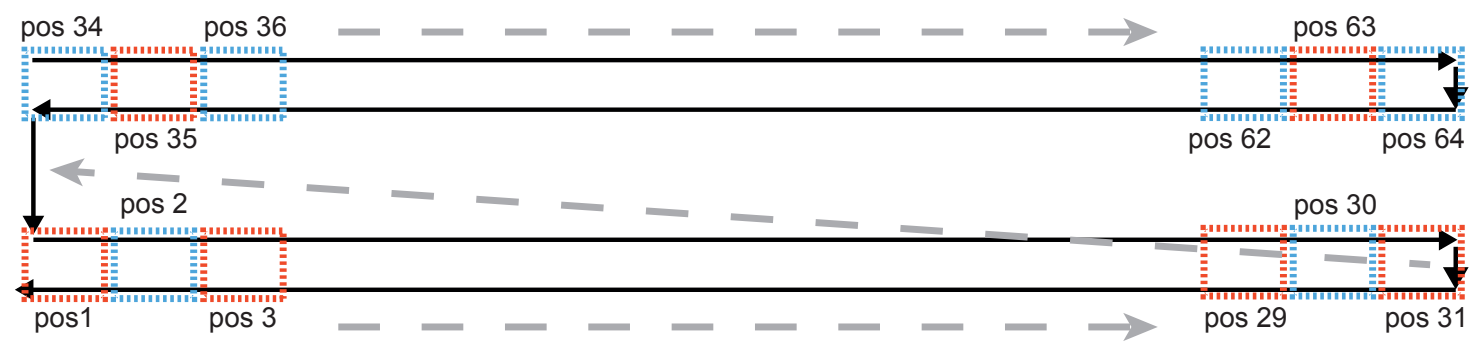

Figure 9.1: Order of the imaging process in device C. We image the drop positions in the experiments in the device $\mathrm{C}$ (Fig. 4.3) with a defined order. The imaging sequence positions are $1,2, \ldots, 64$.

phology is plotted against the drop number (Fig. 7.2c/g). Due to the imaging technique along the serpentine-like shape of the drop storage (Fig. 4.3), the time point of imaging of the drops does not increase linearly with the drop number. Instead, the imaging time for about four drops is sketched by the following concept: The drops with the numbers $(1,2,124,123)$ are imaged together (position 1 in Fig. 9.1), then position $2(3,4,122,121), \ldots$ until the last image at position 31 $(61,62,63,64)$ of the lower double channel is taken. Subsequently, the drops in the top double channel are imaged starting with position $34(127,128,250,249), \ldots$. Following this time axis along Fig. 7.2c/g, there is absolutely no dependence on time. Instead, the drops follow the course of the initial magnesium concentration only. In a worst case scenario, the magnesium concentration is leveled out over all drops over the course of time. Especially the drops with a magnesium concentration that is initially below the vimentin aggregation threshold of $10 \mathrm{mM}$ should take the overall average magnesium concentration of $10 \mathrm{mM}$ over time. Consequently, the filaments in these drops should aggregate over time. However, drops that are imaged at these late times show no aggregation (Fig. 7.2: in c: drop number $\approx 200-230$, in $\mathrm{g}: \approx 200-250$ ). The conclusion from this observation is that any magnesium exchange processes between the drops are so small that they do not influence our results.

For the device T, the observation times are typically below $10 \mathrm{~min}$ (Fig. 4.6). Additionally, over longer times (typically $30 \mathrm{~min}$ ) all produced drops have the same composition. Consequently, exchange phenomena in these experiments are also negligible.

In conclusion, the drop volume changes only have a small influence on the concentrations in the drops. However, the interaction of the protein and the multivalent salt takes place from the very beginning on (Sec. 7.3). Therefore, it is reason- 
able to use the initial salt concentration values for the discussion of the polyelectrolyte nature of vimentin and the competitive binding of the ions in chapter 8 .

\subsubsection{Effects of mixing and inhomogeneity in concentration}

For any interaction of two substances which is concentration dependent, mixing effects are important, since inhomogeneities have the potential to influence the outcome of an experiment. To a large extent, these inhomogeneities are inherent in the system. Whenever two substances are mixed - at least in the very first moment - the concentration might differ locally from the intended equilibrium concentration. There are cases in which this problem can be overcome. For example, caged compounds are used that release one chemical everywhere in the solution, after mixing is perfect (see Sec. 10). Another example is the shrinkage of drops, so that the chemicals homogeneously reach a critical concentration. [57] However, these cases are the exception rather than the rule.

In our microfluidic drops, we also face a concentration inhomogeneity in the very first moments, as we compose the drops of three different components. Small ions are not an issue as their diffusion constant is comparatively large and in the order of $10^{-9} \mathrm{~m}^{2} / \mathrm{s}$ (Sec. 5.1). The slowest diffusing components in our system are the semiflexible vimentin filaments that have an approximate mean contour length in the order of $5-10 \mu \mathrm{m}$. The result is a diffusion constant of $\approx 10^{-12} \mathrm{~m}^{2} / \mathrm{s}$. Consequently, the diffusion constants of the two substances in the drops have a mismatch of three orders of magnitude. When a homogeneous mixing should be achieved, the fast diffusing substance (small ions) must diffuse into the slow diffusing one (protein). For example, this is found for experiments with keratins. [63] In microfluidic channels with one aqueous component the staggered herringbone mixer can also be used to achieve faster mixing. [125] Even in this case, however, a small path always remains, which the molecules have to overcome, before final mixing is achieved. And again, the mismatch in the diffusion constant will be important. For two substances between which the interaction takes place fast - in our case the interaction of multivalent ions and vimentin this can lead to inhomogeneities. For slow interactions, this might not be a challenge at all. In another microfluidic flow focusing based technique used in our group, we also exploit the fact that the ions diffuse into the very slowly diffusing 
protein. Thereby the interaction is studied in a system where the protein concentration is homogeneous in a microfluidic 'jet' in which small ions diffuse into. [26] Our approach to achieve mixing is based on the movement of the plug-like drops in either straight or serpentine channels. In both cases, the mixing of the chemicals greatly outperforms mixing of the content in resting drops (Sec. 5.1). Given this inherent overlay problem of mixing time and interaction time, the mixing in our drops works well. We sometimes observe vimentin morphologies that can be assigned to inhomogeneities in the drop (Sec. 7.11c). Nevertheless, the mixing in the drops should be considered a benefit of the microfluidic technique, since already with drops that have contact to a straight channel the mixing is established in an uncomplicated way and the outcome is, except for minor mixing inhomogeneity, good.

\subsubsection{The challenge of protein aggregation in small channels}

Experimentally, we present microfluidic devices which were designed for a specific task. The key steps of these tasks are: drop production, drop mixing and trapping. The devices we have tailored (chapter 4) prove to be suited for this purpose and work very well for uncritical aqueous solutions, like fluorescent dye solutions or small particles. However, the challenge is the ambitious goal of these microfluidic tools: to study an aggregation process inside the drops. When the protein is finally encapsulated in the drops, the system performs very well because the drop encapsulation reduces the sticking of protein to PDMS or glass surfaces to a large extent. This is an important advantage, as we found no surface coating of comparable performance for vimentin (see also [92]). For F-actin, surface coatings on cover glasses seem to be much more efficient. [57] This might be related to the lower surface charge of actin (Tab. 8.4).

A problem arises before the protein is encapsulated in the drops. At this stage, there is no surfactant layer that shields the proteins from absorption to the channel walls. Additionally, the metal ions that mediate the attraction between the filaments are very small and therefore diffuse fast (Sec. 5.1). Therefore, protein aggregates can grow fast, before being encapsulated, and the channels in front of the drop production often clog during the experiments. Applying the staggered fluid approach (Sec. 5.3) during the device startup, this situation was already considerably improved. Nevertheless, we did not find a method that guarantees 
the success of an experiment.

Looking at this problem within a wider context, this is a general problem when the system length scales are of the same size as the aggregating system. This is often the case for filamentous systems in microfluidic environments. A possible solution to this problem might be the tool of droplet fusion by electrocoalescence (see also Sec. 10). [112] Using this technique, drops that contain multivalent salt could be fused with drops that contain vimentin filament solution. This way, the interaction could be shielded from the microfluidic channel walls. This would reduce the stickiness of the protein in the channels.

An advantage of the problem of channel clogging is that it is easily recognized. Once the protein channel is clogged, the fluorescent protein clog is directly visible and irregularities in the drop production are recognizable. These irregularities can be, for instance, the absence of protein in the drops, polydispersity in the drops size or irregular drop production. Therefore, the diagnosis is precise and we start over with another device, in case of channel clogging. When there is no clogging, the microfluidic tool applied to vimentin fulfills its purpose. In summary, it is not easy to get the devices running. But when the challenges are met, the microfluidic devices provide access to filament observation that is otherwise not accessible (Sec. 9.1.6).

\subsubsection{Are microfluidic drops just miniaturized test tubes?}

In order to conclude the discussion on microfluidic drops as a tool for protein study, we evaluate the most important advantages and disadvantages found in our study.

A drawback of the drops for the study of vimentin is that the microfluidic technique somehow dictates the experiments. In particular, we adjusted our protein concentration in the drops to the possibilities of the microfluidic technique. For instance, Lin et al. perform rheology experiments at a vimentin concentration that is about ten times higher than in our case. [76,77]. However, at such a high concentration the encapsulation of the drops in our devices would lead to severe channel clogging before the protein is encapsulated in the drops. Possibly, a study in much larger microfluidic drops could solve the problem. Nevertheless, many other techniques like electron microscopy, rheology or atomic force microscopy also have limitations concerning the protein concentrations. For in- 
stance, in rheology the concentrations have to be comparatively high, whereas the concentrations in electron microscopy or atomic force microscopy are low. The second conceptual problem is that drops are not necessarily isolated containers (Sec. 9.1.3). In our case, the impact of these effects does not affect the results, but generally, the properties of the emulsion system have to be chosen carefully to be compatible with the system studied in the drops.

These drawbacks are overcompensated by the unique advantages drop studies provide:

Most importantly, we exploit their ability to shield the drop content from the channel walls. This is the best way we know of to prevent vimentin filaments from sticking to the surfaces. The mixing performance of the drop interior is superior to conventional mixing in test tubes. The confinement provided by the small drop volume is important, as the size of the drops fits to the length of filaments and clusters of filaments. Additionally, the confinement is relevant as it mimics the cellular environment. It is possible to define the composition of the drops in a precise way and the overall possibility to manipulate these tiny amounts of samples. These are examples for the high controllability that drops offer. Images taken rapidly after drop composition and over time in the same experiment are only accessible by a drop-based technique.

\subsection{Interaction of vimentin with multivalent ions}

In our experiments, we directly image the influence of multivalent ions on vimentin filaments with respect to concentration, time and valency of the counterions. Furthermore, we investigate the polyelectrolyte nature of vimentin filaments. In this section, we compare experimental observations with theoretical concepts. This sheds light on the understanding of the fundamental principles that the interaction of multivalent ions and vimentin filaments implies. 


\subsubsection{Filament networks}

In both the experiments with the device $C$ and with the device $T$, we find a threshold concentration at $c_{\mathrm{Mg}^{2+}}=10 \mathrm{mM}$, upon which the filaments form networks. In the literature, the molar ratio (MR) that is given as the ratio of magnesium concentration to the protein concentration is discussed for vimentin in the presence of divalent salts. $[69,76]$ For rheology experiments with vimentin, a threshold of network stiffening is observed at $c_{\mathrm{Mg}^{2+}}=4 \mathrm{mM}\left(\mathrm{MR}=205, c_{\text {vimentin }}=1 \mathrm{mg} / \mathrm{mL}\right)$ and the divalent ions are interpreted as 'cross-linkers'. [76] Microrheology studies find an influence of $c_{\mathrm{Mg}^{2+}}=2 \mathrm{mM}\left(\mathrm{MR}=105, c_{\text {vimentin }}=1 \mathrm{mg} / \mathrm{mL}\right)$. [69] For both studies, the threshold concentration is below the threshold we find, but still on the same order of magnitude. There are differences in the preparation method for these systems, as for instance another buffer and sodium chloride instead of potassium chloride is used for filament assembly. Consequently, it is difficult to give a reason for this deviation. When we assume that the preparation does not influence the system, the deviation might have its origin in the different techniques used to study the system. In fluorescence microscopy, we are able to observe morphological changes of the filaments directly. However, it is imaginable that the attraction between the filament becomes stronger at $c_{\mathrm{Mg}^{2+}}=2 \mathrm{mM}$ or $4 \mathrm{mM}$, but when this does not lead to visible changes in fluorescence microscopy, our method is insensitive to these changes. However, with rheology, this kind of inter-filament attraction might be measurable.

In our experiments with the device $C$ and the device $T$, we find the same threshold concentration of magnesium ions for the onset of aggregation. However, as the protein concentrations differ between these two experiments, the molar ratios differ: At the threshold concentration of $c_{\mathrm{Mg}^{2+}}=10 \mathrm{mM}$ for the onset of aggregation, the molar ratio is $\mathrm{MR} \approx 2600$ in the device $\mathrm{C}$ and $\mathrm{MR} \approx 7400$ in the device $\mathrm{T}$. This is a large difference compared to the MR values of the rheology experiments (205 or 105, respectively). From the polyelectrolyte point of view, we consider the interaction of magnesium ions with an individual filament. We expect that potassium ions and magnesium ions compete to bind to the surface of vimentin filaments due to the polyelectrolyte nature of the protein (Sec. 2.2.3). In our rather dilute polymer system (device C: vimentin concentration $0.2 \mathrm{mg} / \mathrm{mL}$; device $\mathrm{T}$ : $0.07 \mathrm{mg} / \mathrm{mL}$ ) the large difference in the MR value might therefore be irrelevant, when the interaction of ions and the vimentin filaments is seen in this way. As a result, the large difference between the molar ratio in the device $C$ and device $T$ 
is expected to be of minor importance. However, when the protein concentration is much higher, it is reasonable to expect other forces to be important. In the discussion here and in the following, we concentrate on the polyelectrolyte aspects, as it is of relevance to vimentin. However, it cannot be excluded that other forces of entropic origin such as depletion forces or hydration forces, are of importance. This may also be the origin of the deviation of our experiments and the rheology studies. But due to its high surface charge (chapter 8 ), the polyelectrolyte nature of vimentin and electrostatic processes are important.

The morphologies of the vimentin networks all show the same trend (Sec. 7). At low ion concentration, the filaments fluctuate freely. Therefore, they spread all over the drops. When a threshold concentration is exceeded, a network of filaments emerges. This network becomes more compact over time. The network morphologies show some heterogeneities, which are likely due to randomly occurring drop internal flows (Sec. 7.3). Basically, we observe scaffold-like or point-like network morphologies. In comparison to the structures we observe in drops (Fig. 7.13e), the networks on the cover glasses are more elongated. An explanation are the drop internal flows, which are circular in the closed drop volume. When a straight bundle emerges, the tip of this bundle might be somehow bended in the presence of a circular flow. As a result, the overall appearance of the networks is more roundish. This effect is also an example for the nature of the filaments we observe: Given a magnesium or hexammine cobalt ion concentration above the threshold, they appear as sticky filaments that attach to each other, when they are in contact. Possibly, there is a reorganization of two filaments that have attached at one point along their contours to a more aligned arrangement, leading the interaction into an energetic minimum. We clearly observe that filaments which are integrated into networks lose their pronounced thermal fluctuations (example in Fig. 7.6). The explanation is that two filaments attract each other and become a bundle or an aggregate of undefined structure. For bundles the persistence length is larger compared to the individual filaments. [79] This explains why the fluctuations of the networks of filaments are much smaller than for individual filaments. The emerging networks are also densified over time. This compaction process is the slowest process in the system (Tab. 7.1). A possible explanation is the process of energy minimization that includes very small reorganization steps in the network. However, these local energy minimization processes lead to a stabilization of the entire network. This way, the network is 
trapped in some cases in a more scaffold-like configuration (example Fig. 7.6). In other cases, the compaction process does not have to overcome such a strong barrier. Therefore, very small networks emerge (Fig. 7.8), which lead to very high protein concentrations in the network structure $\left(c_{\text {vimentin, network }} \approx 30 \mathrm{mg} / \mathrm{mL}\right)$. The persistence length of $2 \mu \mathrm{m}$ of vimentin filaments [93] is likely to act as a barrier against further compaction of the networks at some point.

An important point in these processes is that we do not observe transient clustering of the filaments. It is imaginable that several filaments form a cluster which is spontaneously decomposed by the influence of thermal energy, for example. The reason for this might be that above the threshold the range of attraction forces between the filaments is in general very small (order of magnitude: one nanometer), but the forces are strong. When the threshold concentration could be met extremely precise, this transient cluster formation might be observed. However, we do not observe this state in our experiments. The attraction mechanism of the filaments appears more like double faced adhesive tape, which is of very short range for attraction, but once established, a connection is strong.

An important aspect in our experiments might be the existence of network compaction. A comparison to the behavior of F-actin is relevant: Huber et al. demonstrate that in drops actin filaments form networks at comparable ionic conditions as in our experiment. [57] However, when the expansion range of the networks is compared, there is a fundamental difference. In their example images [57], all F-actin networks occupy the whole drop volume (drop dimension $\approx 100 \mu \mathrm{m}$ ). In contrast, vimentin filaments show a distinct compaction behavior and over time the occupied volume in the drop gets far below the whole drop volume. The larger persistence length of F-actin $(\approx 13 \mu \mathrm{m}$ [70]) compared to vimentin filaments (2 $\mu \mathrm{m}[93])$ might be an important factor in this process.

\subsubsection{The onset of aggregation}

For our experiments with the device $C$ and the device $T$, we find threshold concentrations for magnesium and hexammine cobalt ions upon which the interaction between the vimentin filaments changes qualitatively. For our system parameters (Sec. 8.1), we find experimentally a cation bulk threshold concentration of $10 \mathrm{mM}$ for divalent magnesium ions. For trivalent hexammine cobalt ions, the threshold is below $2.4 \mathrm{mM}$, likely between $0.5 \mathrm{mM}$ and the latter value. 


\section{DISCUSSION}

The problem of the onset of inter-filament attraction is easily described: There are two charged filaments that have the same net negative charge. In vacuum these two filaments would repel each other. [22] What is the reason for the attraction between filaments in a buffer, when a minimal threshold concentration of multivalent ions is present? Considering the discussion on the polyelectrolyte nature of vimentin in chapter 8 , the question is what happens in the system at these thresholds. The threshold concentrations found experimentally are compared to the bulk cation concentrations at which both potassium and the multivalent ions have the same share ('50:50 share') on the filament (Eq. 8.8). The magnesium threshold concentration or the hexammine cobalt threshold concentration are very close to the concentrations at the 50:50 share (Eq. 8.9).

On the basis of the strong correlation between multivalent ions (Sec. 2.2.4 and 8.5), it is clear that monovalent ions alone cannot mediate attraction. When multivalent ions condense onto the filament, attractive forces emerge. As these forces increase with the concentration of multivalent cations on the filament surface, it is likely that this attractive force has to exceed a certain threshold. This way, the attraction force becomes significant compared to the thermal and repulsive forces. Although it is not clear why the share of potassium and the multivalent ion should be $50 \%$, it is clear that the number of condensed multivalent ions is important in this case. Given this comparison between theory and our experimental results, we raise the following hypothesis:

The attraction mechanism leading to the aggregation of vimentin filaments is based on the correlation of multivalent ions which are condensed onto the filaments. In the presence of a significant amount of monovalent cations, the multivalent ions are in competition to the monovalent ions and their fraction on the filament increases with an increased bulk concentration of the multivalent species. A higher fraction on the filament is more easily achieved, when the valency of the multivalent ion species is higher. To mediate effective attraction, the population of the multivalent ions on the filament has to exceed a critical concentration.

The hypothesis is based on the picture of two filaments that are considered below and above the threshold concentration (Fig. 9.2). The hypothesis is built on the following line of arguments. Rouzina and Bloomfield as well as Naji et al. point out the importance of correlations between ions in the process of macro-ion attraction. $[90,106]$ The correlations of the ions in the proximity to the surface of 
a) below threshold

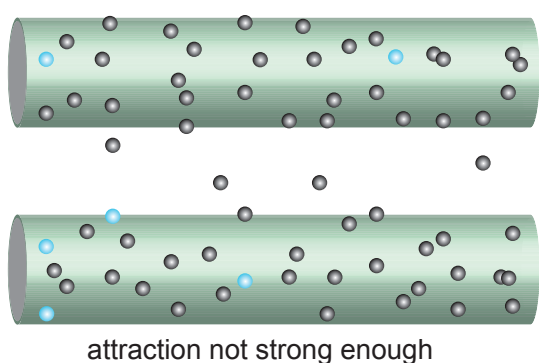

attraction not strong enough

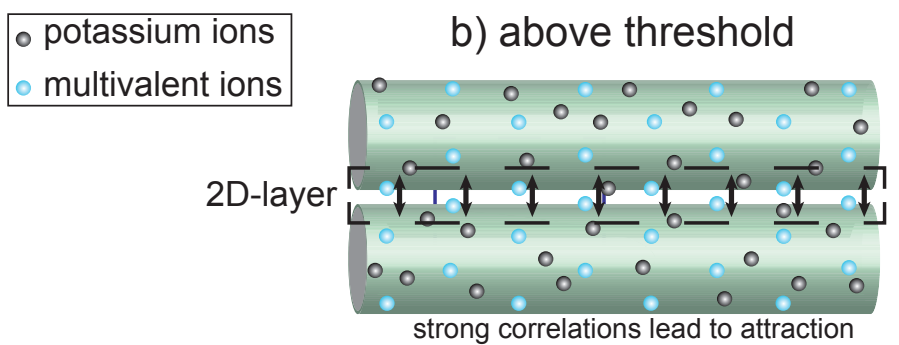

c) attraction in correlation hole

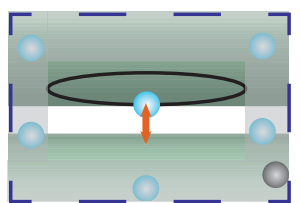

Figure 9.2: Hypothesis for the existence of a threshold concentration. a) When the monovalent potassium ions on the vimentin filaments dominate the population of counterions, the result is that any small attractive forces between the filaments do not lead to (permanent) filament attraction (comp. Fig. 2.8c). The population of the multivalent ions on the surface of the filaments increases as the bulk multivalent ion concentration increases. b) Above a threshold concentration, the correlation ability of multivalent ions dominates the interaction of the counterion population on the filaments. This leads to the attraction of two filaments that come close to each other, as an attractive force emerges (comp. 2.8d). c) In the configuration of a $2 \mathrm{D}$ layer of the magnesium ions between the filaments (see also Fig. 2.8d), the effective interaction partner of the ion is the oppositely charged surface of the neighboring filament. (See also Sec. 2.2.4 and references therein.)

the polyelectrolyte are the main reason for inter-filament attraction. [106] For an effective attraction mechanism, a two-dimensional arrangement of the screening counterions in front of the polyelectrolyte is crucial (Fig. 2.8d and 9.2). [106] A prerequisite for the establishment of a two-dimensional layer of counterions is the strong coupling between the ions. $[90,106]$ The coupling strength increases with the valency of the ions (Tab. 8.4). The lower the bulk magnesium concentration, the lower the magnesium concentration on the surface of the filaments (Fig. 8.2). Below a threshold concentration of magnesium ions, there are either too few magnesium ions to establish an effective two dimensional correlation layer or the absolute number of these ions in the correlation layer is too low. ${ }^{1}$ (Fig. 9.2a). Consequently, the attraction between two filaments that approach each other randomly by diffusion is too weak to be permanent. ${ }^{2}$ These two filaments move apart by random thermal motion. In contrast, in a situation where the bulk concentration of mag-

\footnotetext{
${ }^{1}$ Here, we write statements that are part of our hypothesis in italics. The other statements in this paragraph are directly taken from literature or direct conclusions from literature. [90, 106]

${ }^{2}$ As already pointed out in Sec. 7.3 , the range of attraction forces is in the nanometer range.
} 
nesium ions is high enough, there are lots of magnesium ions in the proximity of the surface of the polyelectrolyte (Fig. 8.2). When these two filaments meet, there are enough ions to correlate and form a 2 D-layer between the two filaments (Fig. $9.2 b)$. Then each relevant multivalent ion 'sits' in a correlation hole of this layer, being bound to one surface and experiencing an attraction to the surface of the other filament (Fig. 9.2c). [90] As a consequence, attraction is established between the filaments and they permanently stick together. A cascade of filament connection events leads to network formation (Sec. 7).

The argument can be transferred to counterions of higher valency, like trivalent hexammine cobalt ions. For these counterions, the ion fraction required on the surface of vimentin for filament aggregation is the lower, the higher the valency of the counterions (Eq. 8.7, Fig. 8.2 and [107]). Consequently, the onset concentration decreases as the valency of the multivalent counterion increases. This is consistent with the experimental finding that the onset concentration of trivalent hexammine cobalt ions is roughly one order of magnitude lower as for divalent magnesium ions (Eq. 8.9).

In the context of DNA condensation, it is often argued that there has to be a certain percentage of the DNA charge neutralized before condensation occurs $(\approx 90 \%)$. [131] This concept implies a consideration of the polyelectrolyte as a cylinder, since a planar surface is always neutralized by bound counterions. [90] The pure calculation for vimentin by the Manning theory as given in Tab. 8.1 predicts that bound monovalent ions alone already neutralized $90 \%$ of the filament charge. However, for potassium concentrations, which are reasonable for filament assembly and stabilization, no network formation or filament bundling is observed. Therefore, we think that the close look into the competitive binding and the interpretation of our experimental results - as we have done - is a reasonable way to understand, why vimentin filaments are aggregating.

A very recent review supports parts of the concept which we present here. It also points out the importance of competitive ion binding to biopolymers and correlations between multivalent ions for the bundling of charged biopolymers. [60] In studies on fd or M13 viruses, which also have distinct polyelectrolyte properties, it is found that the competitive binding of mono- and divalent ions is important for the onset of bundling of the viruses. [132] The importance of competitive binding between mono- and multivalent ions for the onset of aggregation is also found for DNA. It is believed that the ion profiles around the DNA molecules are 
related to the onset of aggregation. [30] As for these polyelectrolytes the concentration of the multivalent ions in the vicinity of the polyelectrolytes is shown to be important for the onset of aggregation, this result is likely to be valid - at least on a qualitative level - also for vimentin. This effect supports the hypothesis we state for the onset of aggregation for vimentin.

It is also of interest to investigate findings on F-actin within the scope of our hypothesis. The concept of competitive binding of ions to polyelectrolytes is a common situation, as buffers of biological samples often contain a significant amount of monovalent ions, which are part of a standard buffer. [105] Like for vimentin in our case, there are other studies of F-actin in which both monovalent and multivalent counterions are present.

At a potassium chloride concentration of $150 \mathrm{mM}$, Tang et al. find bundling of F-actin [131] for divalent ions at threshold concentrations that depend on the specific ions, not only on the valency of the ions. The thresholds found for F-actin bundling are $5.5 \mathrm{mM}$ for cobalt ions $\left(\mathrm{Co}^{2+}\right), 7 \mathrm{mM}$ for manganese ions $\left(\mathrm{Mn}^{2+}\right)$, $20 \mathrm{mM}$ for calcium ions $\left(\mathrm{Ca}^{2+}\right)$ and $27 \mathrm{mM}$ for magnesium ions $\left(\mathrm{Mg}^{2+}\right)$. The authors hypothesize that ionic radii and hydration of ions contribute to this effect. From the polyelectrolyte discussion of this phenomenon, this is a conceptually plausible interpretation of their data, as there is also a link to competitive binding of polyelectrolytes that considers ionic radii. [107] For trivalent hexammine cobalt, the threshold is a found at $c_{\mathrm{Co}\left(\mathrm{NH}_{3}\right)_{6}^{3+}} \approx 3 \mathrm{mM}$. Consequently, this threshold is also below the threshold of all divalent ions studied in the system. For vimentin, this is also the result we observe experimentally. Additionally, it is qualitatively expected based on the polyelectrolyte nature of the proteins (Fig. 8.2). For counterions of valency five and higher (oligomers of arginine, histidine and lysine), the aggregation threshold concentration for F-actin bundling decreases markedly with increasing valency. We expect this trend also for vimentin filaments and it is qualitatively consistent with the expectation on the threshold concentrations in dependence on ion valency (Fig. 8.2).

Tang et al. also investigate the onset of F-actin bundling in dependence of the potassium chloride concentration. Bundling is observed at $c_{\mathrm{Co}\left(\mathrm{NH}_{3}\right)_{6}^{3+}} \approx 0.9,1$, 2 and $3 \mathrm{mM}$ for potassium concentrations of $c_{\mathrm{KCl}}=30,50,100$ and $150 \mathrm{mM}$, respectively. Their explanation is based on a consideration of the ionic strength of the system. $[82,131]$ The observed trend is the same as we would expect by the application of our hypothesis: When the concentration of the monovalent ions is 
increased, the concentration of the trivalent ions has to be increased as well to be significant on the polyelectrolyte. In another publication, Tang et al. state that it is a general behavior that, when the ionic strength (often determined mainly by monovalent ions) is increased, the threshold concentration of the cationic bundling agent also has to be increased for an onset of aggregation. [130] Therefore, the findings of Tang et al. are in good agreement with our hypothesis. Huber et al. find in their study on F-actin that the threshold concentration of magnesium ions for an onset of actin aggregation also increases as the potassium concentration increases. [57] Angelini et al. study actin at $c_{\mathrm{KCl}}=100 \mathrm{mM}$. The network formation onset is observed at $c_{\mathrm{Ba}^{2+}}=6 \mathrm{mM}$, and further different phases of the networks are observed at higher ion concentrations. [5]

In summary, the competitive binding of monovalent and multivalent ions in close vicinity to polyelectrolytes is expected to be of general importance. Studies on other polyelectrolytes support our line of arguments that our hypothesis is based on. When we apply our hypothesis to the observations found in the literature as well as to the findings on vimentin as we just discussed, overall a consistent picture evolves.

\subsubsection{Assumptions for the polyelectrolyte nature}

In chapter 8 , we investigate the polyelectrolyte nature of vimentin. The model we use is based on several assumptions, which are discussed here.

In order to calculate the surface charges of vimentin for a given $\mathrm{pH}$ value, the charges of the amino acid sequence are analyzed. In this analysis, we work with textbook values for the $\mathrm{pK}$-values, which are typical for the amino acids in the protein. However, this has to be seen as an estimation, as the exact $\mathrm{pK}$ value depends on the exact environment of each amino acid (Sec. 8.1). Additionally, we neglect any charges that the fluorescent label can carry. Due to the fact that not all of the protein monomers are labeled, the influence of the label charges is small. Neglecting these small influences, the calculations have a general meaning for vimentin filaments and are not specific for our experiments. All charges from the amino acid sequence lead to a net charge of each vimentin monomer. The resulting line charge density of the filaments is then assumed to be homogeneous. However, there is the pronounced positively charged head region (Fig. 8.2) which could, for instance, be a reason for an inhomogeneous line charge. 
Recent computations on the electrostatic potential maps of vimentin filaments also suggest small stripes of positive charges in the otherwise negatively charged vimentin surface. [60] For our model, however, we assume that charge inhomogeneities level out. This simple approach is considered as the favored one within the mathematical model as the fine details of charge distributions on the surface of a polyelectrolyte could only be accounted for by numerical approaches. [105] We show that moderate deviations from the calculations of the surface charges do not change the central statements we make (Sec. 8.4). As a result, we assume a cylinder-like structure of vimentin, like it is established in a polyelectrolyte discussion of DNA, F-actin and microtubules in the literature (Sec. 2.2).

Vimentin is a semiflexible polymer with a persistence length on the order of $2 \mu \mathrm{m}$ [93], but the length scales relevant for binding of counterions is in the range of nanometers (chapter 8). Consequently, the assumption of vimentin filaments as a stiff rod in the discussion of the polyelectrolyte nature is justified. In summary, the model we apply to vimentin has its weakness and strength in its simplicity: On the one hand, all details of the protein filaments are averaged out. On the other hand, only with this simplification, we are able to apply the formalism of Rouzina and Bloomfield to describe the polyelectrolyte nature of vimentin. [105,107]

With the mathematical descriptions of Rouzina and Bloomfield, the competitive binding of a multivalent salt and a monovalent salt on the charged surface of vimentin is discussed. [105] There are possibilities to account for more details of the system, such as a consideration of ion radii or the consideration of ion species of low concentration (Sec. 8.3) that are also in competitive binding to the surface of vimentin. [107] However, already the most simple system that considers the competition of two assumingly point-like ion types is capable to describe the system to a good extent. In dependence on the ionic strength of the solutions, the radius of the polyelectrolyte and the Gouy-Chapman length, the mathematical regime of the application of the Poisson-Boltzmann equation has to be determined (Sec. 8.1). [105] For each set of system parameters (type of polyelectrolyte, ionic strength,...), the correct mathematical regime has to be chosen. That is why we cannot simply apply our formalism to the studies of F-actin as discussed in Sec. 9.2.2.

The detailed analysis of Rouzina and Bloomfield suggests that the effective geometry (planar vs. cylindrical) of a polyelectrolyte should be chosen based on 
the system parameters. $[105,107]$ Applying the Manning theory to vimentin, we see that the planar geometry is of relevance. Regardless of the exact mathematical description that is needed to consider the competitive binding of ions, their qualitative statement will be the same, although the quantitative results may differ moderately. For instance, when in a polyelectrolyte system the bulk concentration of a divalent ion in addition to a monovalent concentration is increased, the fraction of multivalent ions on the surface of the filament increases. Consequently, it is reasonable to compare our findings for vimentin also to other studies (Sec. 9.2.2).

Our discussion on the onset of aggregation (Sec. 9.2.2) is based on calculations that refer to the non-linear Poisson-Boltzmann equation. [105] We extend our line of argumentation with the concept of strong coupling. With this extension, we take the correlations between ions into account, which are intrinsically neglected in the formalism of the Poisson-Boltzmann equation (Sec. 2.2).

The discussion of the polymorphism of vimentin (Sec. 8.4) leads also to an interesting finding. The polymorphism of vimentin filaments describes the fact that the number of monomers in the cross-section of a unit-length filament (ULF) can be different from the average of 32. [52] In the vicinity of each ULF (length in filament is $43 \mathrm{~nm}$ ), a competitive binding of the ions is expected (Fig. $8.3 \mathrm{c} / \mathrm{d}$ ). When the ULFs are all the same in the filament, an assumingly infinitely long

a) $z_{2}=2 \quad N=28$

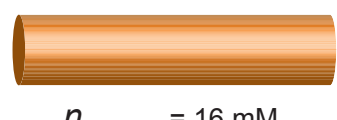

$n_{\mathrm{b} 2,50: 50}=16 \mathrm{mM}$

b) $\mathrm{z}_{2}=2$, at $n_{\mathrm{b} 2}=$ ?

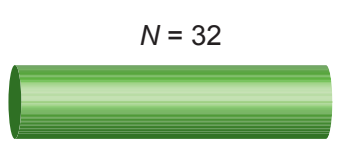

$n_{\mathrm{b} 2,50: 50}=14 \mathrm{mM}$

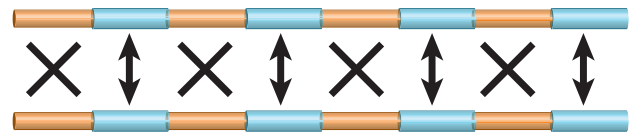

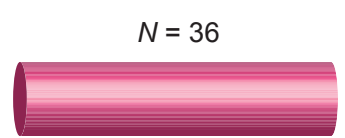

$n_{\mathrm{b} 2,50: 50}=12 \mathrm{mM}$

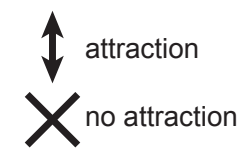

Figure 9.3: Implications of the polymorphism of filament building blocks. a) Different filament fragments of $N$ monomers are given (aspect ratio is to scale, length is the length of a ULF in the filament: $43 \mathrm{~nm}$ ). For $z_{2}=2$ the corresponding $n_{\mathrm{b} 2,50: 50}$ values indicate that on the surface of each fragment type, the competitive binding is different (formalism explained in Sec. 8.3). This could be an interesting phenomenon as for filaments with changing monomer numbers along the filament, the translational invariance in the polyelectrolyte is broken. b) Given certain salt conditions, this could mediate filamentfilament attraction only at certain regions (illustrated with a hypothetical filament). These regions are known for filamentous systems as 'sticky patches'. 
filament would be translational invariant along its contour. Therefore, the interaction with ions and also the attraction mechanisms to other filaments would be the same everywhere. However, in a filament with polymorphism, this symmetry is broken. Therefore, sticky patches for the attraction of different filaments among each other could emerge due to the polyelectrolyte nature of ULFs. This aspect is illustrated in Fig. 9.3. This is of interest for the cross-linking positions in biopolymer networks. There are two types of bonds to be distinguished: 'Transient bonds', at which filaments can connect everywhere along their contour, and 'sticky patches', where a connection is only established at specific positions along the contour. [144]

\subsubsection{The role of the tail}

The role of the tail of intermediate filaments is discussed comprehensively in the literature. $[16,25,52,63,76,77]$ The importance of the tail for inter-filament attraction has been shown for neurofilaments. [16] In this study, the electrostatic interaction of the neurofilament tails is considered to mediate attraction in a handshake like fashion. No explicit role of multivalent ions is considered there. In comparison to vimentin, there is a large difference in the length of the sidearm. [77] Whereas the vimentin tail counts 55 residues, the neurofilament sidearms count 155,526 or 690 residues, depending on the type of the neurofilament protein that is considered. [16] The large difference in the sidearm length suggests that the sidearm is more important to neurofilaments than to vimentin. Nevertheless, a shorter length alone does not have any implication on the importance of the sidearm to vimentin.

In the experiments, mutants of vimentin that lack the last 11 or 55 amino acids of their tail are also built into the networks of full length vimentin (Sec. 7.4.2). We do not observe any difference in the aggregation behavior between the mutant and the full length vimentin within the resolution of fluorescence microscopy. The conclusion is that a hand-shake mechanism - like it is known for neurofilaments - is not necessary to establish a connection to these mutants. However, we cannot measure whether the interaction force is the same for the mutants as for the full length vimentin. This may explain the rheology results which attribute the decrease of the elastic moduli to the lack of parts of the tail. [76]

We give a detailed analysis and discussion of the polyelectrolyte properties of 
the tailless vimentin mutant in Sec. 8.6. In this discussion we find that the tailless mutant of vimentin itself has pronounced electrostatic properties. From the electrostatic point of view, filament attraction is reasonable also without the tail (Sec. 8.6). Based on our experiments as well as the polyelectrolyte discussion, we cannot assign a crucial role to the tail regions for the establishment of interfilament cross-links in the presence of magnesium ions. Additionally, the fact that a mutant consisting of head and rod only can mediate attraction underlines the importance of the polyelectrolyte nature of vimentin.

\subsubsection{Implications for the cytoskeleton}

Our results show the influence of multivalent ions in a purified system in which the number of components is reduced to a few 'players' like the buffer system, protein monomers, a monovalent salt as well as a multivalent salt component. Compared to the gigantic number of 'players' in the cell (Sec. 2.1.2), the complexity of our system is considerably reduced. Nevertheless, the few components show an elaborate interaction that we image directly and also discuss by the consideration of competitive binding. We determine an aggregation threshold of $10 \mathrm{mM}$ for magnesium ions in our experiments. A direct comparison to the available magnesium concentrations in the cell is also available (on the order of $0.8 \mathrm{mM}$; Tab. 2.2). At times, these intracellular magnesium concentrations are likely to be locally higher than $0.8 \mathrm{mM}$. Within this context, a direct transfer of our results to the cell is challenging. As we already reviewed in Sec. 2.1.2, the ionic budget of the cell is fundamentally used for regulation mechanisms of the cell. Therefore, it remains challenging to explain, how networks of cytoskeletal proteins can be regulated directly by small metal ions. Our results shed light on the properties of the intermediate filament vimentin in a fundamental way. Like it has been shown for other fiber-forming cytoskeletal proteins, it has distinct polyelectrolyte properties. The importance of the polyelectrolyte nature of cellular components is pointed out by Janmey et al. for situations like cell death, where these cellular components are spilled into the extracellular environments. There are examples in which the DNA or F-actin form aggregates in the extracellular space and their polyelectrolyte nature leads to complex behavior in extracellular fluids, in which counterions are present. [60] This example shows the general importance of the electrostatic behavior of filament systems like vimentin. As ions 
are everywhere in an organism, the possibility to establish non-specific binding mediated by small ions is omnipresent. These small ions belong to the fastest diffusing particles in the cell and therefore they have the potential to mediate cytoskeletal reorganization in a fast way. It has to be further explored, under which circumstances the electrostatic nature of polyelectrolytes like vimentin is utilized in the body. 



\section{0 \\ Outlook}

When drop-based microfluidic experiments are compared to experiments on cover glasses, both strategies have advantages and disadvantages. One idea would be to combine some of the advantages of both systems. The striking advantage for experiments on the cover glass is their simplicity. One striking advantage of the drops is that they prevent the protein from sticking to the channel walls. The combination of both would address a similar concept as the technique from $\mathrm{Hu}-$ ber et al. (without the shrinkage aspect). [57] The oil-surfactant phase is deposited on a cover glass and the protein solution as well as the salt are injected into the oil phase. This method is experimentally simple and sticking of the protein to the glass might be prevented.

However, this approach alone would not solve the issue of the superposition of network formation and the mixing process of the multivalent salt and the filaments. For this reason, caged ions could be used. The concept of this experimental approach includes that divalent ions are released from another caging molecule upon the irradiation with light. [40] Once the mixing of all components in the drops is perfect, the release of the ions is triggered by light. Then the interaction of these divalent ions with the filaments could be investigated without any influences of the mixing of the drop content.

The hypothesis we raise on the onset of aggregation and therefore also the origin of network formation (Sec. 9.2.2) is in good agreement with our experiments. However, several experiments could further confirm the proposed mechanism as cause for the observed threshold and attraction mechanism. Experiments with ions of higher valency are needed, like the tetravalent spermine ions, which are known for DNA condensation. [21] Following the hypothesis presented in Sec. 
9.2.2, the onset of aggregation for a tetravalent ion in competition with potassium chloride should be about an order of magnitude smaller compared to hexammine cobalt.

Complementary, the importance of competitive ion binding could also be tested for different bulk potassium chloride concentrations, since the filament assembly should not be influenced by a variation of the bulk concentration to a certain extent. [117] Then the competition between the ions on the surface of vimentin is different and the onset of aggregation should take place at another bulk concentration of multivalent ions. Additionally, the influence of the ion radii could be studied with other divalent ions like calcium or manganese. Like it is observed for F-actin, the ionic radii should influence the thresholds for aggregation slightly. [131]

The mechanism we propose as explanation for the onset of aggregation implies that there is a certain threshold concentration of multivalent ions. It would be interesting to find out whether the network formation is reversible. When the bulk concentration of the multivalent ion is initially high enough so that the filaments attract, then a decrease of the bulk concentration afterwards should reverse the network formation. This concept assumes that the filament entanglement is not so strong that the filaments remain entangled in the aggregate. This could be tested in a device, in which a drop that contains a vimentin network is coalesced with a drop that contains filament assembly buffer (no multivalent salt) only (device design like in [42]). During the fusion process of the two drops, the bulk concentration of the multivalent salt solution is diluted. A second method for this reversibility test could be realized with the device of Sun et al., in which the multivalent ion concentration in drops can also be decreased (Sec. 9.1.1).

The exact arrangement of the counterions in a bundle of vimentin filaments could also be investigated by X-ray diffraction studies. In the study of Angelini et al., the organization of counterions in front of F-actin bundles was measured, showing that the ions form a density wave in front of actin. [5] If this detailed data was available for vimentin, this could elucidate the role of divalent ions during bundling for vimentin in detail.

Complementary to the experiments with vimentin, an investigation of other intermediate filaments in the presence of multivalent ions is important. This way the polyelectrolyte properties of intermediate filaments might turn out as a general characteristic of the whole protein family. 


\section{1 \\ Summary and conclusions}

In this study, we developed microfluidic devices that provide the basis for a controlled manipulation of drops. These microfluidic tools enable us to study in detail the aggregation behavior of vimentin filaments in the presence of multivalent ions.

We designed the 'device $C$ ' to investigate the aggregation with respect to changes in the salt concentration. The method is suitable to study the aggregation of vimentin filaments in about 250 drops in parallel at different magnesium chloride concentrations. The drop content can be tuned precisely and all drops are treated the same concerning mixing characteristics and trapping of the drops. The high controllability of the drop content emphasizes the reliability of this microfluidic tool.

We designed the 'device $\mathrm{T}^{\prime}$ to resolve the aggregation behavior of vimentin filaments in the presence of multivalent ions with respect to time. This device meets the challenges to image the content of the drops both rapidly after the first contact of salt and vimentin as well as over a relevant time window of about 10 minutes. Already 1 to 5 seconds after the initial contact of salt and protein, the content of the drops is imaged. Therefore, our technique provides access to the early stages of the filament aggregation process. Additionally, the concept of this device includes the possibility to perform serial time-lapse studies as the content of the next drop can be imaged, once the time-lapse study on its predecessor is finished. This allows a high sample throughput.

For both the device $C$ and the device $T$, the drops are used as shielding containers for vimentin filaments. Vimentin filaments firmly bind to cover glasses - regardless of the presence of multivalent salts. By means of encapsulation of the 
proteins into drops, this interaction is suppressed by the oil-surfactant layer in between the protein filaments and the cover glass. Therefore, the drops are not only a system for which the sample volume is reduced by several orders of magnitude compared to conventional pipetting-based experiments (reduction from micro- to picoliters). Instead, the drops provide highly necessary features for the study of proteins. This shielding effect of the oil-surfactant layer between the protein solution and channel walls is the most effective solution we are aware of. The universal designs of the device $C$ as well as the device $T$ allow for manifold uses. Only slight modifications of the method, if any, will be required to use the devices also for other systems. For instance, F-actin and other intermediate filaments like desmin or keratins could be tested for the dependence of network formation on salts or chemicals. The testing for concentration effects of a chemical as well as time-lapse studies are general challenges in research. Therefore, we expect also various applications in the research fields beyond cytoskeletal proteins, such as cell research or drug discovery.

In the microfluidic drop experiments, we directly image the interaction of vimentin filaments and threshold concentrations of salt ions for the onset of aggregation. The experiments with both the device $C$ and device $T$ show that magnesium ions induce aggregation of vimentin filaments to networks. The prerequisite for the network formation process is a critical threshold concentration of magnesium ions of about $10 \mathrm{mM}$. Below this threshold concentration the filaments show thermal motion only. They fluctuate continuously within the drops. Consequently, any attractive inter-filament force that is mediated by the magnesium ions is - if existing - too small to lead to a permanent association of the filaments. Therefore, thermal motion dominates this process. However, we observe the formation of filaments to networks above this magnesium threshold concentration.

The dynamics of this network formation process are accessible in the device T. For a magnesium concentration above the threshold, we find that the network formation process starts immediately when the filaments and ions get in contact. From this perspective, the accessible observation time in device $\mathrm{T}$ meets the time scales that are relevant for the network formation of vimentin. Most other techniques used for the studies on cytoskeletal proteins in the presence of multivalent salt provide measurements on the equilibrium state of the proteins. The advantage of our method is the accessibility of the aggregation process towards an equilibrium 
state.

The time scale of aggregation is determined by the interplay of different diffusion processes. Directly after the productions of the drops, we estimate that small magnesium ions are distributed all over the drop. Consequently, it is consistent that the aggregation process starts early, when the interaction between the filaments is mediated by these ions. Our investigation of the polyelectrolyte properties of vimentin leads to the conclusion that the range of forces is approximately one nanometer or below. Therefore, a prerequisite for an inter-filament attraction is the close proximity of neighboring filaments. Two filaments can come close by diffusion. Consequently, this filament diffusion determines also the time-scale of aggregation. Additionally, flows can accelerate the aggregation process, as they transport filaments. These annealing mechanisms are important, only then the forces between two filaments are present. This principle evokes the picture of filaments that become sticky in the presence of multivalent ions by short-ranged attractive force.

The small ions lead to the formation of sophisticated vimentin networks. When the networks of filaments have already established, its inherent stability slows down morphological changes in the networks. For further morphological changes, small internal reorganization processes of the filaments are expected to be necessary. The stabilization process of the network also explains that the compaction of the networks is slow compared to early network formation processes. Nevertheless, the density of the filament in the networks can increase drastically. Our experiments therefore attest that small ions have the potential to mediate a very strong overall interaction between vimentin filaments.

In addition to our experiments performed with divalent magnesium ions, we also tested the aggregation behavior of vimentin in the presence of the trivalent hexammine cobalt ions. These ions also lead to aggregation of vimentin, once a threshold concentration is exceeded. However, the threshold concentration of about 0.5-2.4 $\mathrm{mM}$ is roughly one order of magnitude lower compared to magnesium ions. This behavior is understood within the context of the competitive binding of ions to filaments (see below).

On a more fundamental level, we identify the polyelectrolyte nature of vimentin as the reason for the aggregation in the presence of multivalent salts. We raise the hypothesis that the competitive binding of counterions to vimentin filaments is the reason for the existence of a threshold concentration for the onset of aggrega- 
tion. This model is appealing, as it provides a quantitative criterion for the onset of aggregation. At the onset of aggregation, the fraction of multivalent ions on the vimentin filaments reaches a critical value. Due to the ability of multivalent ions to effectively correlate, they form a two dimensional layer, which leads to the attraction of two vimentin filaments.

The order of magnitude of the threshold concentration found in the experiments and by the theoretical calculation agrees well. The experiments show that the threshold concentrations for aggregation are smaller for multivalent ions of higher valency. This aspect is found in our studies as well as in the literature. The corresponding effect is directly found in competitive binding, where the fraction of the multivalent ions on the surface of the filament also depends strongly on the valency of the multivalent counterion.

The investigations of the polyelectrolyte properties of vimentin introduce a new aspect to the discussion of the role of the vimentin tail. The analysis of the polyelectrolyte nature of tail-truncated vimentin filaments suggests that - from the polyelectrolyte point of view - the tail is not important to mediate the interaction between vimentin filaments in the presence of magnesium ions. Instead, we find that it is possible for vimentin to mediate attraction without the tail, since the head-rod complex of vimentin itself has distinct polyelectrolyte properties. This theoretical analysis corresponds to our qualitative analysis of composite networks of vimentin and tail-truncated vimentin.

In conclusion, magnesium and hexammine cobalt ions are capable to mediate strong aggregation effects on vimentin filaments. The onset of aggregation mediated by multivalent ions is also reported in the literature for other biopolymers. We present a hypothesis that has the potential to explain this onset for vimentin filaments and possibly also for other fiber-forming cytoskeletal proteins in which the standard buffers used for filament formation contain monovalent salts. Multivalent ions are then added and the aggregation sets in once a critical concentration is exceeded. Further studies should be carried out with a focus on the competitive binding of counterions. We suggest to apply the basic principle of the calculation presented here for vimentin to other biopolymers to obtain a prediction for the onset of aggregation. For vimentin, the calculations for the onset of aggregation and the experimental findings are consistent. This agreement underlines the importance of the polyelectrolyte nature of vimentin filaments. The results are likely to be transferable to other intermediate filaments. 


\section{Abbreviations and symbols}

\section{Abbreviations}

$\begin{array}{ll}\text { aq 1, aq 2, aq } 3 & \begin{array}{l}\text { aqueous component } 1,2 \text { and } 3 \text { that compose the aque- } \\ \text { ous drops (Fig. } 4.1 \text { and } 4.4) \\ \text { adenosine triphosphate }\end{array} \\ \text { ATP } & \begin{array}{l}\text { bovine serum albumin } \\ \text { BSA }\end{array} \\ \text { CCAL } & \begin{array}{l}\text { Creative Commons Attribution License } \\ \text { device to test for concentration dependence (Sec. } 4.1)\end{array} \\ \text { device C } & \text { device to test for time dependence (Sec. } 4.2) \\ \text { device T } & \text { dithiothreitol } \\ \text { DTT } & \text { ethylenediaminetetraacetic acid } \\ \text { EDTA } & \text { ethylene glycol tetraacetic acid } \\ \text { EGTA } & \text { equation } \\ \text { Eq. } & \text { filamentous actin } \\ \text { F-actin } & \text { figure } \\ \text { Fig. } & \text { guanosine triphosphate } \\ \text { GTP } & \text { conserved intensity: parameter for convex hull analy- } \\ \text { CI } & \text { sis (Sec. } 6.2 .3) \\ \text { IF(s) } & \text { intermediate filament(s) } \\ \text { IFAP(s) } & \text { intermediate filament associated protein(s) } \\ \text { MAP } & \text { microtubules associated proteins } \\ \text { MT(s) } & \text { microtubules } \\ \text { MWCO } & \text { molecular weight cut-off } \\ \text { PB } & \text { phosphate buffer (typically at } p \mathrm{H}=7.5) \\ \text { PDMS } & \text { polydimethylsiloxane } \\ \text { Tab. } & \text { table }\end{array}$


TRIS

ULF(s)

'vimentin' tris (hydroxymethyl) aminomethane

unit length filament(s) (basic building blocks of vimentin filaments)

vimentin mutant with an amino acid sequence very

close to human vimentin wild-type (see Sec. 3.1.1)

\section{Most important symbols}

Here, the most important symbols are listed. The meaning of a symbol that is not listed becomes clear from the context of its occurrence in the text. If applicable, typical values for our experiments are provided.

\begin{tabular}{|c|c|}
\hline$\Delta C 411$ & mutant of wild-type vimentin protein, see Sec. 3.1.1 \\
\hline$\Delta C 455$ & mutant of wild-type vimentin protein, see Sec. 3.1.1 \\
\hline$\Theta_{z}$ & $\begin{array}{l}\text { fraction of filament charge screened by counterions of } \\
\text { valency } z\end{array}$ \\
\hline$\lambda$ & $\begin{array}{l}\text { linear charge density of a polyelectrolyte (Fig. 2.6; for } \\
\text { vimentin filaments: }-14 \frac{e}{\mathrm{~nm}} \text { (Eq. 8.3)) }\end{array}$ \\
\hline$\mu$ & $\begin{array}{l}\text { Gouy-Chapman length (Eq. 2.21); values for different } \\
\text { biopolymers: Tab. } 8.4\end{array}$ \\
\hline$\xi$ & $\begin{array}{l}\text { 'Manning parameter': charges per Bjerrum length, } \\
\xi:=l_{\mathrm{B}} / b \text { (Eq. 2.12). Vimentin filaments: } \xi=9.9 \text { (Eq. } \\
\text { 8.5) }\end{array}$ \\
\hline$\Xi$ & $\begin{array}{l}\text { electrostatic coupling parameter (Eq. 2.23); values for } \\
\text { different biopolymers: Tab. } 8.4\end{array}$ \\
\hline & surface charge density (vimentin: $0.071 \mathrm{C} \mathrm{m}^{-2}$ ) \\
\hline$\sigma_{\mathrm{I}}$ & $\begin{array}{l}\text { standard deviation of intensity values in a drop (Sec. } \\
7.1 \text { ) }\end{array}$ \\
\hline$b$ & $\begin{array}{l}\text { spacing of charges along a 'linear' filament (Fig. 2.6) } \\
\text { (vimentin filaments: } 0.0714 \mathrm{~nm} \text { (Eq. 8.4)) }\end{array}$ \\
\hline$c_{\mathrm{Co}\left(\mathrm{NH}_{3}\right)_{6}^{3+}}$ & $\begin{array}{l}\text { bulk concentration of hexammine cobalt ions } \\
\text { (typical: } 0 \ldots 9.6 \mathrm{mM} \text { ) }\end{array}$ \\
\hline init & initial concentration \\
\hline$c_{\mathrm{KCl}}$ & $\begin{array}{l}\text { bulk concentration of potassium chloride } \\
\text { (always: } 100 \mathrm{mM} \text { ) }\end{array}$ \\
\hline+ & $\begin{array}{l}\text { bulk concentration of magnesium ions } \\
\text { (typical: } 0 \ldots 18.6 \mathrm{mM} \text { ) }\end{array}$ \\
\hline
\end{tabular}




\begin{tabular}{|c|c|}
\hline$c_{\text {syr }}$ & concentration in the syringe \\
\hline $\mathrm{Co}\left(\mathrm{NH}_{3}\right)_{6} \mathrm{Cl}_{3}$ & hexammine cobalt-III-chloride \\
\hline$e$ & elementary charge \\
\hline E & penalty function (Eq. 6.3) \\
\hline$D$ or $D_{X}$ & diffusion constant (of $X$ ) \\
\hline$k_{\mathrm{B}}$ & Boltzmann constant \\
\hline$l_{\mathrm{B}}$ & $\begin{array}{l}\text { Bjerrum-length (in water at room temperature } \\
0.71 \mathrm{~nm} \text { ) }\end{array}$ \\
\hline$l_{\mathrm{D}}$ & Debye-length (typical: $\approx 1 \mathrm{~nm}$ ) \\
\hline$L$ & distance or filament length \\
\hline $\mathrm{L}_{p}$ & persistence length (vimentin: $\approx 2 \mu \mathrm{m}[93]$ ) \\
\hline $\mathrm{MgCl}_{2}$ & magnesium chloride \\
\hline$N$ & $\begin{array}{l}\text { number of experiments } / \text { number of protein } \\
\text { monomers in the filament cross section }\end{array}$ \\
\hline$n_{\mathrm{b} i}$ & 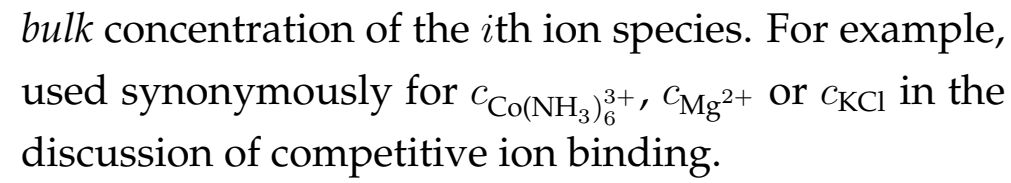 \\
\hline$n_{i}$ & local concentration of the $i$ th ion species \\
\hline$n_{\mathrm{si}}$ & $\begin{array}{l}\text { concentration of the } i \text { th ion species of valency } z_{i} \text { on the } \\
\text { surface of a polyelectrolyte }\end{array}$ \\
\hline$\tilde{n}_{\mathrm{s} i}$ & $\begin{array}{l}n_{\mathrm{si}} \text { normalized to } n_{\mathrm{s}} \text { : fraction compared to all counter- } \\
\text { ions of the } i \text { th ion species on the surface of a polyelec- } \\
\text { trolyte }\end{array}$ \\
\hline$n_{s}$ & $\begin{array}{l}\text { concentration of counterions in close vicinity to a } \\
\text { polyelectrolyte (vimentin: } 1.5 \mathrm{M} \text { ) }\end{array}$ \\
\hline$P e$ & Péclet number (Eq. 2.29) \\
\hline$R$ & radius of a biopolymer (vimentin: $5 \mathrm{~nm}$ ) \\
\hline$t$ & time \\
\hline$t_{\mathrm{d}}$ & delay time \\
\hline$t_{k}$ & time point, when the $k$ th drop is collected \\
\hline$T$ & temperature (typical: room temperature) \\
\hline$u_{X}$ & $\begin{array}{l}\text { flow velocity (channel average) of the component } X \text {. } \\
\text { (device C: Tab. 4.1, device T: Tab. 4.2) }\end{array}$ \\
\hline$V_{r}(t)$ & drop volume at time $t$ normalized to $V_{r}(t=0)$ \\
\hline
\end{tabular}


quantity that parameterizes 'bulk' concentrations in competitive ion binding (Eq. 2.20)

$z_{i}$ or $z$ valency (of the $i$ th ion) 


\section{Bibliography}

[1] P. Abbyad, R. Dangla, A. Alexandrou, and C. N. Baroud. Rails and anchors: Guiding and trapping droplet microreactors in two dimensions. Lab on a Chip, 11:813-821, 2011. (Cited on page 24)

[2] B. Alberts, A. Johnson, J. Lewis, M. Raff, K. Roberts, and P. Walter. Molecular Biology of the Cell. Garland Science, 5th edition, 2008. (Cited on pages 5, 6, and 7)

[3] J. Anastassopoulou and T. Theophanides. Magnesium-DNA interactions and the possible relation of magnesium to carcinogenesis. Irradiation and free radicals . Critical Reviews in Oncology/Hematology, 42(1):79-91, 2002. (Cited on page 17)

[4] K. Andresen, R. Das, H. Y. Park, H. Smith, L. W. Kwok, J. S. Lamb, E. J. Kirkland, D. Herschlag, K. D. Finkelstein, and L. Pollack. Spatial distribution of competing ions around DNA in solution. Physical Review Letters, 93:248103, 2004. (Cited on page 19)

[5] T. Angelini, H. Liang, W. Wriggers, and G. Wong. Direct observation of counterion organization in F-actin polyelectrolyte bundles. The European Physical Journal E, 16(4):389-400, 2005. (Cited on pages 12, 132, and 140)

[6] T. E. Angelini, H. Liang, W. Wriggers, and G. C. L. Wong. Like-charge attraction between polyelectrolytes induced by counterion charge density waves. Proceedings of the National Academy of Sciences, 100(15):8634-8637, 2003. (Cited on page 19)

[7] S. L. Anna, N. Bontoux, and H. A. Stone. Formation of dispersions using 'flow focusing' in microchannels. Applied Physics Letters, 82(3):364-366, 2003. (Cited on pages $23,40,41$, and 46) 
[8] E. N. Archive. Homo sapiens vimentin gene. Online Database. http: //www.ebi.ac.uk/, accessed: 2013-10-14. (Cited on page 29)

[9] R. C. Arevalo, J. S. Urbach, and D. L. Blair. Size-dependent rheology of type-I collagen networks. Biophysical Journal, 99(8):L65-L67, 2010. (Cited on page 75)

[10] Y. Bai, X. He, D. Liu, S. N. Patil, D. Bratton, A. Huebner, F. Hollfelder, C. Abell, and W. T. S. Huck. A double droplet trap system for studying mass transport across a droplet-droplet interface. Lab on a Chip, 10:12811285, 2010. (Cited on page 24)

[11] J.-C. Baret. Surfactants in droplet-based microfluidics. Lab on a Chip, 12:422433, 2012. (Cited on page 23)

[12] J.-C. Baret, O. J. Miller, V. Taly, M. Ryckelynck, A. El-Harrak, L. Frenz, C. Rick, M. L. Samuels, J. B. Hutchison, J. J. Agresti, D. R. Link, D. A. Weitz, and A. D. Griffiths. Fluorescence-activated droplet sorting (FADS): Efficient microfluidic cell sorting based on enzymatic activity. Lab on a Chip, 9:1850-1858, 2009. (Cited on pages 23 and 24)

[13] J.-L. Barrat and F. Joanny. Theory of Polyelectrolyte Solutions, pages 1-66. John Wiley \& Sons, Inc., 1996. (Cited on page 20)

[14] D. Bartolo, G. Degre, P. Nghe, and V. Studer. Microfluidic stickers. Lab on a Chip, 8:274-279, 2008. (Cited on page 24)

[15] A. Bausch and K. Kroy. A bottom-up approach to cell mechanics. Nature Physics, 2(4):231-238, 2006. (Cited on page 6)

[16] R. Beck, J. Deek, J. B. Jones, and C. R. Safinya. Gel-expanded to gelcondensed transition in neurofilament networks revealed by direct force measurements. Nature Materials, 9(1):40-46, 2009. (Cited on pages 11, 99, 113, and 135)

[17] D. J. Beebe, G. A. Mensing, and G. M. Walker. Physics and applications of microfluidics in biology. Annual Review of Biomedical Engineering, 4(1):261286, 2002. PMID: 12117759. (Cited on page 22)

[18] S. H. Behrens and D. G. Grier. The charge of glass and silica surfaces. The Journal of Chemical Physics, 115:6716, 2001. (Cited on page 117) 
[19] J. M. Berg, J. L. Tymoczko, and L. Stryer. Stryer Biochemie. Spektrum, 6th edition, 2007. (Cited on page 103)

[20] E. Berthier, E. W. K. Young, and D. Beebe. Engineers are from PDMS-land, biologists are from polystyrenia. Lab on a Chip, 12:1224-1237, 2012. (Cited on pages 24 and 56)

[21] V. A. Bloomfield. Condensation of DNA by multivalent cations: Considerations on mechanism. Biopolymers, 31(13):1471-1481, 1991. (Cited on pages 12 and 139)

[22] V. A. Bloomfield. DNA condensation by multivalent cations. Biopolymers, 44(3):269-282, 1997. (Cited on pages 12, 13, 17, and 128)

[23] D. Boal. Mechanics of the Cell. Cambridge University Press, 2002. (Cited on page 14)

[24] M. Brennich. Cation induced self-assembly of intermediate filaments. PhD thesis, Niedersächsische Staats-und Universitätsbibliothek Göttingen, 2013. (Cited on page 103)

[25] M. E. Brennich, S. Bauch, U. Vainio, T. Wedig, H. Herrmann, and S. Köster. Impact of ion valency on the assembly of vimentin studied by quantitative small angle X-ray scattering. Soft Matter, 2014. (Cited on pages 10, 99, and 135)

[26] M. E. Brennich, J.-F. Nolting, C. Dammann, B. Nöding, S. Bauch, H. Herrmann, T. Pfohl, and S. Köster. Dynamics of intermediate filament assembly followed in micro-flow by small angle X-ray scattering. Lab on a Chip, 11:708-716, 2011. (Cited on page 122)

[27] M. R. Bringer, C. J. Gerdts, H. Song, J. D. Tice, and R. F. Ismagilov. Microfluidic systems for chemical kinetics that rely on chaotic mixing in droplets. Philosophical Transactions of the Royal Society A, 362(1818):1087-1104, 2004. (Cited on pages 26, 41, 42, 46, and 47)

[28] E. Brouzes, M. Medkova, N. Savenelli, D. Marran, M. Twardowski, J. B. Hutchison, J. M. Rothberg, D. R. Link, N. Perrimon, and M. L. Samuels. Droplet microfluidic technology for single-cell high-throughput screening. Proceedings of the National Academy of Sciences, 106(34):14195-14200, 2009. (Cited on page 23) 
[29] H. Bruus. Theoretical microfluidics. Oxford University Press, 2008. (Cited on pages 22,25 , and 26)

[30] Y. Burak, G. Ariel, and D. Andelman. Onset of DNA aggregation in presence of monovalent and multivalent counterions. Biophysical Journal, 85(4):2100-2110, 2003. (Cited on page 131)

[31] F. Capito, R. Skudas, B. Stanislawski, and H. Kolmar. Polyelectrolyte - protein interaction at low ionic strength: Required chain flexibility depending on protein average charge. Colloid and Polymer Science, 291(7):1759-1769, 2013. (Cited on page 103)

[32] A. A. Chernyatina, S. Nicolet, U. Aebi, H. Herrmann, and S. V. Strelkov. Atomic structure of the vimentin central $\alpha$-helical domain and its implications for intermediate filament assembly. Proceedings of the National Academy of Sciences, 109(34):13620-13625, 2012. (Cited on page 29)

[33] P. A. Coulombe, O. Bousquet, L. Ma, S. Yamada, and D. Wirtz. The 'ins' and 'outs' of intermediate filament organization. Trends in Cell Biology, 10(10):420 - 428, 2000. (Cited on page 7)

[34] C. Dammann. Microfluidic drops as tunable bio-environments. Diploma Thesis, Georg-August-Universität Göttingen. 2010. (Cited on pages 29, 39, 43, and 65)

[35] C. Dammann and S. Köster. Dynamics of counterion-induced attraction between vimentin filaments followed in microfluidic drops. Lab Chip, accepted for publication May 2014, DOI:10.1039/C3LC51418H. (Cited on pages $35,45,46,71,74,76,82,87,89,90$, and 91 )

[36] C. Dammann, B. Nöding, and S. Köster. Vimentin networks at tunable ion-concentration in microfluidic drops. Biomicrofluidics, 6(2):022009, 2012. (Cited on pages $40,41,79$, and 80 )

[37] D. Di Carlo. Inertial microfluidics. Lab on a Chip, 9:3038-3046, 2009. (Cited on page 23)

[38] C. G. Dos Remedios, D. Chhabra, M. Kekic, I. V. Dedova, M. Tsubakihara, D. A. Berry, and N. J. Nosworthy. Actin binding proteins: Regulation of cy- 
toskeletal microfilaments. Physiological Reviews, 83(2):433-473, 2003. (Cited on page 7)

[39] D. Duffy, J. McDonald, O. Schueller, and G. Whitesides. Rapid prototyping of microfluidic systems in poly(dimethylsiloxane). Analytical Chemistry, 70(23):4974-4984, 1998. (Cited on page 34)

[40] G. C. Ellis-Davies. Caged compounds: Photorelease technology for control of cellular chemistry and physiology. Nature Methods, 4(8):619-628, 2007. (Cited on page 139)

[41] J. E. Eriksson, T. Dechat, B. Grin, B. Helfand, M. Mendez, H.-M. Pallari, and R. D. Goldman. Introducing intermediate filaments: From discovery to disease. The Journal of Clinical Investigation, 119(7):1763-1771, 2009. (Cited on pages 6 and 7 )

[42] H. M. Evans, E. Surenjav, C. Priest, S. Herminghaus, R. Seemann, and T. Pfohl. In situ formation, manipulation, and imaging of dropletencapsulated fibrin networks. Lab on a Chip, 9:1933-1941, 2009. (Cited on pages 23,118 , and 140)

[43] A. Fallah-Araghi, J.-C. Baret, M. Ryckelynck, and A. D. Griffiths. A completely in vitro ultrahigh-throughput droplet-based microfluidic screening system for protein engineering and directed evolution. Lab on a Chip, 12:882-891, 2012. (Cited on pages 23 and 24)

[44] F. Fogolari, A. Brigo, and H. Molinari. The Poisson-Boltzmann equation for biomolecular electrostatics: A tool for structural biology. Journal of Molecular Recognition, 15(6):377-392, 2002. (Cited on page 13)

[45] E. Fuchs and K. Weber. Intermediate filaments: Structure, dynamics, function and disease. Annual Review of Biochemistry, 63(1):345-382, 1994. (Cited on page 9)

[46] K. J. Green, M. Böhringer, T. Gocken, and J. C. Jones. Intermediate filament associated proteins. Advances in Protein Chemistry, 70:143-202, 2005. (Cited on page 7) 
[47] A. Y. Grosberg, T. T. Nguyen, and B. I. Shklovskii. Colloquium: The physics of charge inversion in chemical and biological systems. Reviews of Modern Physics, 74:329-345, Apr 2002. (Cited on pages 15, 19, 20, and 117)

[48] P. Gruner and J.-C. Baret. Personal communication. Max Planck Institute for Dynamics and Self-Organization, Göttingen, Germany, 2013. (Cited on page 119)

[49] M. Guéron and G. Weisbuch. Polyelectrolyte theory. I. Counterion accumulation, site-binding, and their insensitivity to polyelectrolyte shape in solutions containing finite salt concentrations. Biopolymers, 19(2):353-382, 1980. (Cited on page 18)

[50] H. Herrmann. Personal communication. DKFZ Heidelberg, Germany. (Cited on page 31)

[51] H. Herrmann and U. Aebi. Intermediate filaments: Molecular structure, assembly mechanism, and integration into functionally distinct intracellular scaffolds. Annual Review of Biochemistry, 73(1):749-789, 2004. (Cited on pages 92 and 112)

[52] H. Herrmann, M. Häner, M. Brettel, S. A. Müller, K. N. Goldie, B. Fedtke, A. Lustig, W. W. Franke, and U. Aebi. Structure and assembly properties of the intermediate filament protein vimentin: The role of its head, rod and tail domains. Journal of Molecular Biology, 264(5):933-953, 1996. (Cited on pages $9,10,29,99,109,134$, and 135)

[53] H. Herrmann, L. Kreplak, and U. Aebi. Isolation, characterization, and in vitro assembly of intermediate filaments. In M. B. Omary and P. A. Coulombe, editors, Intermediate Filament Cytoskeleton, volume 78 of Methods in Cell Biology, pages 3 - 24. Academic Press, 2004. (Cited on page 32)

[54] H. Herrmann, S. V. Strelkov, P. Burkhard, and U. Aebi. Intermediate filaments: Primary determinants of cell architecture and plasticity. The Journal of Clinical Investigation, 119(7):1772-1783, 7 2009. (Cited on pages 6, 7, and 10)

[55] C. Holtze, A. C. Rowat, J. J. Agresti, J. B. Hutchison, F. E. Angilè, C. H. J. Schmitz, S. Köster, H. Duan, K. J. Humphry, R. A. Scanga, J. S. Johnson, 
D. Pisignano, and D. A. Weitz. Biocompatible surfactants for water-influorocarbon emulsions. Lab on a Chip, 8:1632-1639, 2008. (Cited on page 37)

[56] F. Huber, J. Schnauß, S. Rönicke, P. Rauch, K. Müller, C. Fütterer, and J. Käs. Emergent complexity of the cytoskeleton: From single filaments to tissue. Advances in Physics, 62(1):1-112, 2013. (Cited on page 11)

[57] F. Huber, D. Strehle, and J. Käs. Counterion-induced formation of regular actin bundle networks. Soft Matter, 8:931-936, 2012. (Cited on pages 12, 60, 112, $121,122,127,132$, and 139)

[58] A. Huebner, D. Bratton, G. Whyte, M. Yang, A. J. deMello, C. Abell, and F. Hollfelder. Static microdroplet arrays: A microfluidic device for droplet trapping, incubation and release for enzymatic and cell-based assays. Lab on a Chip, 9:692-698, 2009. (Cited on pages 24, 46, and 47)

[59] J. N. Israelachvili. Intermolecular and surface forces: Revised third edition. Academic Press, 2011. (Cited on pages 13 and 22)

[60] P. Janmey, D. Slochower, Y.-H. Wang, Q. Wen, and A. Cebers. Polyelectrolyte properties of filamentous biopolymers and their consequences in biological fluids. Soft Matter, 2014. (Cited on pages 9, 11, 13, 104, 130, 133, and 136)

[61] G. D. M. Jeffries, J. S. Kuo, and D. T. Chiu. Controlled shrinkage and reexpansion of a single aqueous droplet inside an optical vortex trap. The Journal of Physical Chemistry B, 111(11):2806-2812, 2007. (Cited on page 24)

[62] L. Kaufman, C. Brangwynne, K. Kasza, E. Filippidi, V. Gordon, T. Deisboeck, and D. Weitz. Glioma expansion in collagen I matrices: Analyzing collagen concentration-dependent growth and motility patterns. Biophysical Journal, 89(1):635-650, 2005. (Cited on page 75)

[63] J. Kayser, H. Grabmayr, M. Harasim, H. Herrmann, and A. R. Bausch. Assembly kinetics determine the structure of keratin networks. Soft Matter, 8:8873-8879, 2012. (Cited on pages 75, 99, 121, and 135)

[64] S. Kim and P. A. Coulombe. Intermediate filament scaffolds fulfill mechanical, organizational, and signaling functions in the cytoplasm. Genes $\mathcal{E} D e-$ velopment, 21(13):1581-1597, 2007. (Cited on page 6) 
[65] S. Kim and P. A. Coulombe. Emerging role for the cytoskeleton as an organizer and regulator of translation. Nature Reviews Molecular Cell Biology, 11(1):75-81, 2010. (Cited on pages 5 and 7)

[66] A. Kis, S. Kasas, B. Babić, A. Kulik, W. Benoit, G. Briggs, C. Schönenberger, S. Catsicas, and L. Forro. Nanomechanics of microtubules. Physical Review Letters, 89:248101, Nov 2002. (Cited on page 6)

[67] S. Köster, F. E. Angilè, H. Duan, J. J. Agresti, A. Wintner, C. H. J. Schmitz, A. C. Rowat, C. A. Merten, D. Pisignano, A. D. Griffiths, and D. A. Weitz. Drop-based microfluidic devices for encapsulation of single cells. Lab on a Chip, 8:1110-1115, 2008. (Cited on pages 23 and 24)

[68] S. Köster, A. Evilevitch, M. Jeembaeva, and D. A. Weitz. Influence of internal capsid pressure on viral infection by phage $\lambda$. Biophysical Journal, 97(6):1525-1529, 2009. (Cited on page 23)

[69] S. Köster, Y.-C. Lin, H. Herrmann, and D. A. Weitz. Nanomechanics of vimentin intermediate filament networks. Soft Matter, 6:1910-1914, 2010. (Cited on pages 11 and 125)

[70] S. Köster, H. Stark, T. Pfohl, and J. Kierfeld. Fluctuations of single confined actin filaments. Biophysical Reviews and Letters, 2(02):155-166, 2007. (Cited on pages 6 and 127)

[71] J. R. Lakowicz. Principles of fluorescence spectroscopy. Springer, 3rd edition, 2006. (Cited on page 69)

[72] F. Lang, G. Busch, M. Ritter, H. Völkl, S. Waldegger, E. Gulbins, and D. Häussinger. Functional significance of cell volume regulatory mechanisms. Physiological Reviews, 78(1):247-306, 1998. (Cited on page 8)

[73] C. L. Leung, K. J. Green, and R. K. Liem. Plakins: A family of versatile cytolinker proteins. Trends in Cell Biology, 12(1):37-45, 2002. (Cited on page 7)

[74] G. Li and J. X. Tang. Diffusion of actin filaments within a thin layer between two walls. Physical Review E, 69:061921, 2004. (Cited on page 52)

[75] J. W. Lichtman and J.-A. Conchello. Fluorescence microscopy. Nature Methods, 2(12):910-919, 2005. (Cited on page 37) 
[76] Y.-C. Lin, C. P. Broedersz, A. C. Rowat, T. Wedig, H. Herrmann, F. C. MacKintosh, and D. A. Weitz. Divalent cations crosslink vimentin intermediate filament tail domains to regulate network mechanics. Journal of Molecular Biology, 399(4):637 - 644, 2010. (Cited on pages 11, 31, 99, 112, 123, 125, and 135)

[77] Y.-C. Lin, N. Y. Yao, C. P. Broedersz, H. Herrmann, F. C. MacKintosh, and D. A. Weitz. Origins of elasticity in intermediate filament networks. Physical Review Letters, 104(5):058101, 2010. (Cited on pages 11, 99, 123, and 135)

[78] H. Lodish. Molecular Cell Biology. W. H. Freeman, 5th edition, 2004. (Cited on pages $7,8,29$, and 103)

[79] L. Ma, S. Yamada, D. Wirtz, and P. A. Coulombe. A 'hot-spot' mutation alters the mechanical properties of keratin filament networks. Nature Cell Biology, 3(5):503-506, 2001. (Cited on page 126)

[80] E. Mandelkow and E.-M. Mandelkow. Microtubules and microtubuleassociated proteins. Current Opinion in Cell Biology, 7(1):72 - 81, 1995. (Cited on page 7)

[81] G. S. Manning. Limiting laws and counterion condensation in polyelectrolyte solutions I. Colligative properties. The Journal of Chemical Physics, 51(3):924-933, 1969. (Cited on page 15)

[82] G. S. Manning. The molecular theory of polyelectrolyte solutions with applications to the electrostatic properties of polynucleotides. Quarterly Reviews of Biophysics, 11(02):179-246, 1978. (Cited on page 131)

[83] G. S. Manning. Counterion binding in polyelectrolyte theory. Accounts of Chemical Research, 12(12):443-449, 1979. (Cited on page 16)

[84] D. Meschede. Gerthsen Physik, 22. Aufl., 2004. (Cited on page 66)

[85] MicroChem. SU-8 3000 Datasheet. Newton, USA, http://microchem.com/ Prod-SU83000.htm, access on 10.09.2013. (Cited on page 35)

[86] M. Molteni, D. Magatti, B. Cardinali, M. Rocco, and F. Ferri. Fast twodimensional bubble analysis of biopolymer filamentous networks pore size from confocal microscopy thin data stacks. Biophysical Journal, 104(5):11601169, 2013. (Cited on page 75) 
[87] N. Mücke, T. Wedig, A. Bürer, L. N. Marekov, P. M. Steinert, J. Langowski, U. Aebi, and H. Herrmann. Molecular and biophysical characterization of assembly-starter units of human vimentin. Journal of Molecular Biology, 340(1):97-114, 2004. (Cited on pages 10 and 118)

[88] M. Müller, S. S. Bhattacharya, T. Moore, Q. Prescott, T. Wedig, H. Herrmann, and T. M. Magin. Dominant cataract formation in association with a vimentin assembly disrupting mutation. Human Molecular Genetics, 18(6):1052-1057, 2009. (Cited on page 6)

[89] S. Münster and B. Fabry. A simplified implementation of the bubble analysis of biopolymer network pores. Biophysical Journal, 104(12):2774-2775, 2013. (Cited on page 75)

[90] A. Naji, S. Jungblut, A. G. Moreira, and R. R. Netz. Electrostatic interactions in strongly coupled soft matter. Physica A: Statistical Mechanics and its Applications, 352(1):131-170, 2005. (Cited on pages 17, 20, 21, 106, 111, 112, 128, 129, and 130)

[91] D. J. Needleman, M. A. Ojeda-Lopez, U. Raviv, H. P. Miller, L. Wilson, and C. R. Safinya. Higher-order assembly of microtubules by counterions: From hexagonal bundles to living necklaces. Proceedings of the National Academy of Sciences of the United States of America, 101(46):16099-16103, 2004. (Cited on pages 12 and 19)

[92] B. Nöding. Mechanics of Intermediate Filaments. PhD thesis, Georg-AugustUniversität Göttingen, 2014. (Cited on page 122)

[93] B. Nöding and S. Köster. Intermediate filaments in small configuration spaces. Physical Review Letters, 108:088101, 2012. (Cited on pages 10, 90, 118, 127,133 , and 147)

[94] M. B. Omary. 'IF-pathies': A broad spectrum of intermediate filamentassociated diseases. The Journal of Clinical Investigation, 119(7):1756-1762, 7 2009. (Cited on page 6)

[95] F. Oosawa. Polyelectrolytes. Marcel Dekker, 1971. (Cited on page 15) 
[96] R. R. Pompano, W. Liu, W. Du, and R. F. Ismagilov. Microfluidics using spatially defined arrays of droplets in one, two, and three dimensions. Annual Review of Analytical Chemistry, 4:59-81, 2011. (Cited on page 24)

[97] S. Portet, N. Mücke, R. Kirmse, J. Langowski, M. Beil, and H. Herrmann. Vimentin intermediate filament formation: In vitro measurement and mathematical modeling of the filament length distribution during assembly. Langmuir, 25:8817-8823, 2009. (Cited on pages 10, 106, and 110)

[98] A. Priel and J. Tuszyński. A nonlinear cable-like model of amplified ionic wave propagation along microtubules. Europhysics Letters, 83(6):68004, 2008. (Cited on page 16)

[99] E. M. Purcell. Life at low Reynolds number. American Journal of Physics, 45(1), 1977. (Cited on page 23)

[100] Z. Qin, L. Kreplak, and M. J. Buehler. Hierarchical structure controls nanomechanical properties of vimentin intermediate filaments. PLOS ONE, 4(10):e7294, 10 2009. (Cited on page 9)

[101] S. R. Quake and A. Scherer. From micro- to nanofabrication with soft materials. Science, 290:1536-1540, 2000. (Cited on page 34)

[102] E. Raspaud, M. O. de la Cruz, J.-L. Sikorav, and F. Livolant. Precipitation of DNA by polyamines: A polyelectrolyte behavior. Biophysical Journal, 74(1):381-393, 1998. (Cited on page 12)

[103] A. J. Ridley. Life at the leading edge. Cell, 145(7):1012-1022, 2011. (Cited on page 5)

[104] K. R. Rogers, H. Herrmann, and W. W. Franke. Characterization of disulfide crosslink formation of human vimentin at the dimer, tetramer, and intermediate filament levels. Journal of Structural Biology, 117(1):55-69, 1996. (Cited on page 31)

[105] I. Rouzina and V. A. Bloomfield. Competitive electrostatic binding of charged ligands to polyelectrolytes: Planar and cylindrical geometries. The Journal of Physical Chemistry, 100(10):4292-4304, 1996. (Cited on pages 17, 18, $19,105,131,133$, and 134) 
[106] I. Rouzina and V. A. Bloomfield. Macroion attraction due to electrostatic correlation between screening counterions. 1. Mobile surface-adsorbed ions and diffuse ion cloud. The Journal of Physical Chemistry, 100(23):9977-9989, 1996. (Cited on pages 128 and 129)

[107] I. Rouzina and V. A. Bloomfield. Competitive electrostatic binding of charged ligands to polyelectrolytes: Practical approach using the nonlinear Poisson-Boltzmann equation. Biophysical Chemistry, 64(1-3):139-155, 1997. (Cited on pages 17, 18, 19, 106, 130, 131, 133, and 134)

[108] V. Schaller, K. M. Schmoller, E. Karakose, B. Hammerich, M. Maier, and A. R. Bausch. Crosslinking proteins modulate the self-organization of driven systems. Soft Matter, 9:7229-7233, 2013. (Cited on page 6)

[109] C. H. J. Schmitz, A. C. Rowat, S. Köster, and D. A. Weitz. Dropspots: A picoliter array in a microfluidic device. Lab on a Chip, 9(1):44-49, 2009. (Cited on pages $23,24,41,43$, and 115 )

[110] M. Schopferer, H. Bär, B. Hochstein, S. Sharma, N. Mücke, H. Herrmann, and N. Willenbacher. Desmin and vimentin intermediate filament networks: Their viscoelastic properties investigated by mechanical rheometry. Journal of Molecular Biology, 388(1):133-143, 2009. (Cited on page 118)

[111] H. Schwertz, S. Köster, W. H. A. Kahr, N. Michetti, B. F. Kraemer, D. A. Weitz, R. C. Blaylock, L. W. Kraiss, A. Greinacher, G. A. Zimmerman, and A. S. Weyrich. Anucleate platelets generate progeny. Blood, 115(18):38013809, 2010. (Cited on page 23)

[112] R. Seemann, M. Brinkmann, T. Pfohl, and S. Herminghaus. Droplet based microfluidics. Reports on Progress in Physics, 75(1):016601, 2012. (Cited on pages 23,24 , and 123)

[113] J. U. Shim, G. Cristobal, D. R. Link, T. Thorsen, and S. Fraden. Using microfluidics to decouple nucleation and growth of protein crystals. Crystal Growth E Design, 7(11):2192-2194, 2007. (Cited on page 24)

[114] L. Shui, A. Berg, and J. C. Eijkel. Scalable attoliter monodisperse droplet formation using multiphase nano-microfluidics. Microfluidics and Nanofluidics, 11:87-92, 2011. (Cited on page 23) 
[115] M. G. Simon, R. Lin, J. S. Fisher, and A. P. Lee. A Laplace pressure based microfluidic trap for passive droplet trapping and controlled release. Biomicrofluidics, 6(1):014110, 2012. (Cited on page 24)

[116] Y. Skhiri, P. Gruner, B. Semin, Q. Brosseau, D. Pekin, L. Mazutis, V. Goust, F. Kleinschmidt, A. El Harrak, J. B. Hutchison, E. Mayot, J.-F. Bartolo, A. D. Griffiths, V. Taly, and J.-C. Baret. Dynamics of molecular transport by surfactants in emulsions. Soft Matter, 8:10618-10627, 2012. (Cited on pages 24 and 119)

[117] A. V. Sokolova, L. Kreplak, T. Wedig, N. Mücke, D. I. Svergun, H. Herrmann, U. Aebi, and S. V. Strelkov. Monitoring intermediate filament assembly by small-angle $X$-ray scattering reveals the molecular architecture of assembly intermediates. Proceedings of the National Academy of Sciences, 103(44):16206-16211, 2006. (Cited on pages 104 and 140)

[118] H. Song and R. F. Ismagilov. Millisecond kinetics on a microfluidic chip using nanoliters of reagents. Journal of the American Chemical Society, 125(47):14613-14619, 2003. (Cited on pages 40,41, 45, 46, and 118)

[119] T. M. Squires and S. R. Quake. Microfluidics: Fluid physics at the nanoliter scale. Reviews of Modern Physics, 77:977-1026, Oct 2005. (Cited on page 25)

[120] F. A. Steinböck and G. Wiche. Plectin: A cytolinker by design. Biological Chemistry, 380(2):151-158, 1999. (Cited on pages 7 and 8)

[121] P. Steinert, L. Marekov, and D. Parry. Diversity of intermediate filament structure. Evidence that the alignment of coiled-coil molecules in vimentin is different from that in keratin intermediate filaments. Journal of Biological Chemistry, 268(33):24916-24925, 1993. (Cited on page 104)

[122] D. Steinhauser, S. Köster, and T. Pfohl. Mobility gradient induces crossstreamline migration of semiflexible polymers. ACS Macro Letters, 1(5):541545, 2012. (Cited on page 56)

[123] D. Stigter. Evaluation of the counterion condensation theory of polyelectrolytes. Biophysical Journal, 69(2):380-388, 1995. (Cited on page 15)

[124] H. Stone, A. Stroock, and A. Ajdari. Engineering flows in small devices. Annual Review of Fluid Mechanics, 36(1):381-411, 2004. (Cited on page 27) 
[125] A. D. Stroock, S. K. W. Dertinger, A. Ajdari, I. Mezić, H. A. Stone, and G. M. Whitesides. Chaotic mixer for microchannels. Science, 295(5555):647-651, 2002. (Cited on pages 25 and 121)

[126] M. Sun, S. S. Bithi, and S. A. Vanapalli. Microfluidic static droplet arrays with tuneable gradients in material composition. Lab on a Chip, 11:39493952, 2011. (Cited on pages 24 and 116)

[127] M. Sun and S. A. Vanapalli. Generation of chemical concentration gradients in mobile droplet arrays via fragmentation of long immiscible diluting plugs. Analytical Chemistry, 85(4):2044-2048, 2013. (Cited on page 24)

[128] I. Szeverenyi, A. J. Cassidy, C. W. Chung, B. T. Lee, J. E. Common, S. C. Ogg, H. Chen, S. Y. Sim, W. L. Goh, K. W. Ng, J. A. Simpson, L. L. Chee, G. H. Eng, B. Li, D. P. Lunny, D. Chuon, A. Venkatesh, K. H. Khoo, W. I. McLean, Y. P. Lim, and E. B. Lane. The human intermediate filament database: Comprehensive information on a gene family involved in many human diseases. Human Mutation, 29(3):351-360, 2008. (Cited on pages 6 and 7)

[129] C. Tanford and J. G. Kirkwood. Theory of protein titration curves. I. General equations for impenetrable spheres. Journal of the American Chemical Society, 79(20):5333-5339, 1957. (Cited on page 103)

[130] J. X. Tang, T. Ito, T. Tao, P. Traub, and P. A. Janmey. Opposite effects of electrostatics and steric exclusion on bundle formation by F-actin and other filamentous polyelectrolytes. Biochemistry, 36(41):12600-12607, 1997. (Cited on pages 11,12 , and 132)

[131] J. X. Tang and P. A. Janmey. The polyelectrolyte nature of F-actin and the mechanism of actin bundle formation. Journal of Biological Chemistry, 271(15):8556-8563, 1996. (Cited on pages 11, 14, 16, 112, 130, 131, and 140)

[132] J. X. Tang, P. A. Janmey, A. Lyubartsev, and L. Nordenskiöld. Metal ioninduced lateral aggregation of filamentous viruses $\mathrm{fd}$ and M13. Biophysical Journal, 83(1):566-581, 2002. (Cited on pages 51 and 130)

[133] G. Taylor. Dispersion of soluble matter in solvent flowing slowly through a tube. Proceedings of the Royal Society of London. Series A. Mathematical and Physical Sciences, 219(1137):186-203, 1953. (Cited on page 25) 
[134] J. D. Tice, H. Song, A. D. Lyon, and R. F. Ismagilov. Formation of droplets and mixing in multiphase microfluidics at low values of the Reynolds and the capillary numbers. Langmuir, 19(22):9127-9133, 2003. (Cited on pages 53, 56, and 118)

[135] D. Toivola, P. Strnad, A. Habtezion, and M. Omary. Intermediate filaments take the heat as stress proteins. Trends in Cell Biology, 20(2):79-91, 2010. (Cited on page 6)

[136] J. R. C. van der Maarel. Introduction to biopolymer physics. World Scientific, 2008. (Cited on pages 13 and 14)

[137] S. A. Vanapalli, M. H. G. Duits, and F. Mugele. Microfluidics as a functional tool for cell mechanics. Biomicrofluidics, 3(1):012006, 2009. (Cited on page 22)

[138] P. Vanysek. Ionic conductivity and diffusion at infinite dilution. CRC Handbook of Chemistry and Physics, 83, 1999. (Cited on page 51)

[139] Q. Wen and P. A. Janmey. Polymer physics of the cytoskeleton. Current Opinion in Solid State and Materials Science, 15(5):177-182, 2011. (Cited on page 109)

[140] G. Wiche. Role of plectin in cytoskeleton organization and dynamics. Journal of Cell Science, 111(17):2477-2486, 1998. (Cited on pages 7 and 8)

[141] R. Wilson, D. Rau, and V. Bloomfield. Comparison of polyelectrolyte theories of the binding of cations to DNA. Biophysical Journal, 30(2):317-325, 1980. (Cited on page 16)

[142] R. Windoffer, S. Wöll, P. Strnad, and R. E. Leube. Identification of novel principles of keratin filament network turnover in living cells. Molecular Biology of the Cell, 15(5):2436-2448, 2004. (Cited on page 5)

[143] S. Winheim, A. R. Hieb, M. Silbermann, E.-M. Surmann, T. Wedig, H. Herrmann, J. Langowski, and N. Mücke. Deconstructing the late phase of vimentin assembly by total internal reflection fluorescence microscopy (TIRFM). PLOS ONE, 6(4):e19202, 2011. (Cited on page 32)

[144] L. Wolff, P. Fernandez, and K. Kroy. Inelastic mechanics of sticky biopolymer networks. New Journal of Physics, 12(5):053024, 2010. (Cited on page 135) 
[145] G. C. Wong and L. Pollack. Electrostatics of strongly charged biological polymers: Ion-mediated interactions and self-organization in nucleic acids and proteins. Annual Review of Physical Chemistry, 61:171-189, 2010. (Cited on pages $11,13,19$, and 22)

[146] G. C. L. Wong, A. Lin, J. X. Tang, Y. Li, P. A. Janmey, and C. R. Safinya. Lamellar phase of stacked two-dimensional rafts of actin filaments. Physical Review Letters, 91:018103, Jul 2003. (Cited on page 12)

[147] B. Zheng, J. D. Tice, L. S. Roach, and R. F. Ismagilov. A droplet-based, composite PDMS/glass capillary microfluidic system for evaluating protein crystallization conditions by microbatch and vapor-diffusion methods with on-chip X-ray diffraction. Angewandte Chemie International Edition, 43(19):2508-2511, 2004. (Cited on page 23) 


\section{List of publications}

- Christian Dammann and Sarah Köster:

Dynamics of counterion-induced attraction between vimentin filaments followed in microfluidic drops, Lab Chip, 2014, DOI:10.1039/C3LC51418H.

- B. Weinhausen, O. Saldanha, R. N. Wilke, C. Dammann, M. Priebe, M. Burghammer, M. Sprung, and S. Köster:

Scanning X-ray Nano-Diffraction of Living Eukaryotic Cells in Microfluidic Environments, Physical Review Letters 112 (2014) 088102.

- Christian Dammann, Bernd Nöding and Sarah Köster:

Vimentin Networks at Tunable Ion-Concentration in Microfluidic Drops Biomicrofluidics 6 (2012), 022009.

- Martha E. Brennich, Jens-F. Nolting, Christian Dammann, Bernd Nöding, Susanne Bauch, Harald Herrmann, Thomas Pfohl and Sarah Köster:

Dynamics of intermediate filament assembly followed in micro-flow by small angle x-ray scattering, Lab on a Chip 11 (2011), 708-716. 



\section{Erklärung}

Hiermit erkläre ich, dass ich diese Dissertation selbständig angefertigt habe und keine anderen Hilfsmittel oder Quellen als die angegebenen benutzt habe. Alle Stellen, die ich wörtlich oder sinngemäß aus anderen Schriften entnommen habe, habe ich als solche gekennzeichnet.

Darüber hinaus erkläre ich, dass ich diese Dissertation weder ganz noch in Teilen an einer anderen Hochschule in irgendeiner Form eingereicht habe.

Göttingen, den 12.2.2014

Christian Dammann 



\section{Lebenslauf}

\section{Christian Dammann}

geboren am 3.10.1985 in Stade, Deutschland.

\section{Schulischer und wissenschaftlicher Werdegang}

seit $08 / 2010$

$08 / 2010$

$10 / 2005-$

$08 / 2010$

$06 / 2005$
Promotionsstudent an der "Göttingen Graduate School for Neurosciences, Biophysics, and Molecular Biosciences (GGNB)“. Wissenschaftlicher Mitarbeiter zunächst am „Courant Research Centre“ und später am Institut für Röntgenphysik, Georg-August-Universität Göttingen.

Diplom in Physik an der Georg-August-Universität Göttingen. Diplomarbeit „Microfluidic Drops as Tunable BioEnvironments" angefertigt im "Courant Research Centre" der Georg-August- Universität Göttingen.

Diplomstudiengang Physik an der Georg-AugustUniversität Göttingen

Erlangung der allgemeinen Hochschulreife an der Halepaghen-Schule Buxtehude 



\section{Danksagung}

Viele Menschen haben zum Gelingen meiner Doktorarbeit durch Diskussionen, Anregungen und Kommentare oder auch der Bereitstellung von Materialien beigetragen. An dieser Stelle möchte ich mich dafür herzlich bedanken!

Mein besonderer Dank gilt Prof. Dr. Sarah Köster, dass sie mir die Möglichkeit gegeben hat, in ihrer Arbeitsgruppe an meiner Promotion zu arbeiten. Sie hat sich stets für mich Zeit genommen und hatte immer ein offenes Ohr für neue Ideen. Die gemeinsamen wissenschaftlichen Diskussionen waren stets zielführend. Ich bin auch dankbar, dass ich an mehreren Messzeiten an Synchrotron-Einrichtungen teilnehmen konnte. Die Atmosphäre innerhalb dieser großen Forschungseinrichtungen habe ich stets als sehr inspirierend empfunden.

Ich möchte mich auch bei meinen Thesis Committee Mitgliedern bedanken. Prof. Dr. Eberhard Bodenschatz und Dr. Thomas Burg haben meine Promotion begleitet und mit ihren Anregungen in den Thesis Commitee Meetings das Projekt vorangebracht.

Ich danke Prof. Dr. Sarah Köster und Dr. Thomas Burg ebenfalls für die Begutachtung meiner Dissertation und Prof. Dr. Jean-Christophe Baret, Prof. Dr. Jörg Enderlein und Dr. Florian Rehfeldt, dass sie meiner Prüfungskommission angehören.

Dem SFB 755 „Nanoscale Photonic Imaging“ danke ich für die finanzielle Unterstützung meines Projekts.

Ich danke Prof. Dr. Harald Herrmann-Lerdon, Norbert Mücke, Tatjana Wedig und Stefan Winheim vom DKFZ in Heidelberg für die Bereitstellung der Vimentin Plasmide und die vielen anregenden Diskussionen zu Intermediärfilamenten. Ich danke Susanne Bauch für die Herstellung und Aufreinigung der Proteine. 
Ich danke Philipp Gruner und Prof. Dr. Jean-Christophe Baret für die Diskussionen zu mikrofluidischen Themen.

Für die Bereitstellung von Surfactants bin ich der Firma Raindance Technologies und insbesondere Brian Hutchison dankbar.

Meinen Bürokollegen Susanne Bauch, Bernd Nöding, Jens Nolting, Rabea Sandmann und Dr. Britta Weinhausen danke ich herzlich für viele interessante Diskussionen und die wundervolle Büroatmosphäre.

Für Korrekturlesearbeiten möchte ich mich bei Dr. Rita Graceffa, Clément Hémonnot, Bernd Nöding, Jens Nolting, Aishwarya Paknikar, Oliva Saldanha, Viktor Schroeder, Dr. Britta Weinhausen und besonders bei Rabea Sandmann bedanken.

Ich danke dem gesamten Institut für Röntgenphysik für die freundliche Arbeitsatmosphäre. Insbesondere möchte ich mich bei Jan Goemann und Jochen Herbst bedanken, die technische Probleme immer schnell und zuverlässig gelöst haben. Auch möchte ich mich bei meinen ehemaligen Kollegen Dr. Martha Brennich, Jannick Langpfahl-Klabes und Dr. Sarah Schwarz für die gute Zusammenarbeit bedanken.

Meinen Studienfreunden Britta, Katha, Julian, Mira, Patrick, Steffi und Thilo danke ich für viele schöne gemeinsame Jahre in Göttingen.

Ich danke meinen Eltern Gunda und Erich Dammann sowie Heide Hofmann für sorgsame Ermunterungen. Meiner Magdalena danke ich von ganzem Herzen für die liebevolle Unterstützung in all den Jahren. 